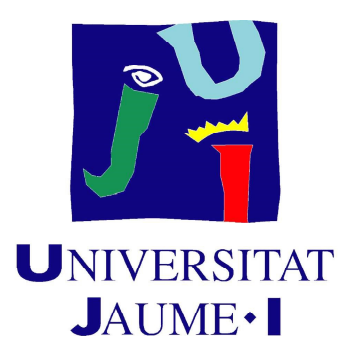

DOCTORAL THESIS

METHODS FOR THE DESIGN AND EVALUATION OF ANTHROPOMORPHIC ARTIFICIAL HANDS

\author{
Author: Immaculada Llop Harillo
}

Supervisor: Dr. Antonio Pérez González 



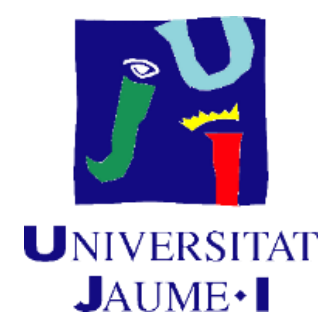

DOCTORAL THESIS

\title{
METHODS FOR THE DESIGN AND EVALUATION OF ANTHROPOMORPHIC ARTIFICIAL HANDS
}

\author{
Author: \\ Immaculada Llop Harillo
}

Supervisor:

Dr. Antonio Pérez González

Castellón (Spain), June 2020 



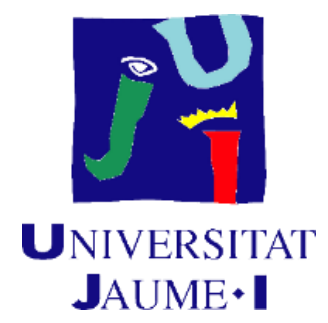

Doctoral Programme in Industrial Technologies and Materials Universitat Jaume I Doctoral School

Methods for the design and evaluation of anthropomorphic artificial hands

Report submitted by Immaculada Llop Harillo in order to be eligible for a doctoral degree awarded by the Universitat Jaume I

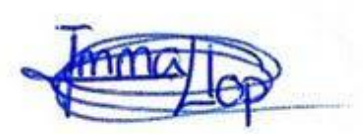

Immaculada Llop Harillo

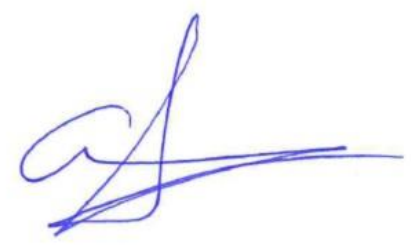

Antonio Pérez González

Castelló de la Plana, June 2020 



\section{Funding}

Spanish Ministry of Economy and Competitiveness and ESF with a FPIMINECO predoctoral contract 2016-2020 (grant number BES-2015076005)

Spanish Ministry of Economy and Competitiveness and ERDF (project grant number DPI2014-60635-R)

Spanish Ministry of Economy and Competitiveness, AEI and ERDF (project grant number DPI2017-89910-R)

Universitat Jaume I (project grant number UJI-B2017-70) 



\section{Thesis by compendium of publications:}

1. Immaculada Llop-Harillo and Antonio Pérez-González (2017) System for the experimental evaluation of anthropomorphic hands. Application to a new 3Dprinted prosthetic hand prototype, International Biomechanics, 4:2, 50-59, DOI: 10.1080/23335432.2017.1364666, SJR 0.553 (Q2)

2. Immaculada Llop-Harillo, Antonio Pérez-González, Julia Starke, and Tamim Asfour (2019) The Anthropomorphic Hand Assessment Protocol (AHAP),

Robotics and Autonomous Systems, vol.121, DOI: 10.1016/j.robot.2019.103259, JCR 2.928 (Q2), SJR 0.831 (Q1)

3. Immaculada Llop-Harillo, Antonio Pérez-González, and F. Javier Andrés, Grasping ability and motion synergies in affordable tendon-driven prosthetic hands controlled by able-bodied subjects. Submitted to Frontiers in Neurorobotics, JCR 3.000 (Q2), SJR 0.603 (Q2)

4. Immaculada Llop-Harillo, Antonio Pérez-González, and Verónica Gracia-Ibáñez (2019) Anthropomorphism Index of Mobility for Artificial Hands, Applied Bionics and Biomechanics, vol. 2019, 1-11, DOI: 10.1155/2019/7169034, JCR 1.525 (Q3), SJR 0.346 (Q3)

5. Immaculada Llop-Harillo, Antonio Pérez-González, and Javier AndrésEsperanza (2020) Anthropomorphism Indexes of the Kinematic Chain for Artificial Hands, Journal of Bionic Engineering, vol. 17, 501-511, DOI: 10.1007/s42235-020-0040-5, JCR 2.463 (Q2), SJR 0.609 (Q2)

6. Antonio Pérez-González and Immaculada Llop-Harillo (2020) Optimization of the Kinematic Chain of the Thumb for a Hand Prosthesis Based on the Kapandji Opposition Test. In: Ateshian G., Myers K., Tavares J. (eds) Computer Methods, Imaging and Visualization in Biomechanics and Biomedical Engineering. CMBBE 2019. Lecture Notes in Computational Vision and Biomechanics, vol. 36, 271-287. Springer, Cham. DOI: 10.1007/978-3-030-43195-2_22

7. Immaculada Llop-Harillo, José L. Iserte, and Antonio Pérez-González, Benchmarking prosthetic hands through anthropomorphic grasping simulations. Submitted to Bioinspiration \& Biomimetics, JCR 3.130 (Q1), SJR 1.000 (Q1)

This thesis has been accepted by the co-authors of the publications listed above that have waved the right to present them as a part of another PhD thesis 

"... the modern human brain came after the hominid hand..."

Anthropologist Sherwood Washburn 

The objective of this thesis is to contribute to the definition of applicable methods during the design process of artificial hands in order to obtain more anthropomorphic and functional designs, as well as to define metrics and protocols that help evaluate these aspects. The proposed methods are focused on the mechanical design of any anthropomorphic hand, both in the prosthetic and robotic fields, although in this thesis greater emphasis has been placed on hands designed for 3D printing, given its growing popularity and accessibility and its potential impact on society. Most of the developments and proposals made are based on biomechanical aspects obtained from the analysis of the human hand. In this thesis, the evaluation of the degree of anthropomorphism of artificial hands has been approached from three complementary perspectives: experimentation, definition of analytical indexes and simulation by means of models.

Firstly, a prosthetic hand (IMMA hand) with six independently actuated cables/tendons has been designed and a first prototype has been manufactured using 3D printing. For the experimental evaluation of this, or other tendon-driven hands, a device (able-bodied adaptor, ABA) has been designed allowing the tendons actuation through the fingers of a healthy subject. This ABA allows to record the excursion in each tendon, enabling the posterior analysis of actuation synergies. This information is interesting for the design of the hand actuation and/or its control system.

An experimental protocol, called Anthropomorphic Hand Assessment Protocol (AHAP), has been defined for the evaluation of the anthropomorphism and the functionality of artificial hand prototypes. This protocol includes the eight most common grasp types of the human hand, apart from two non-prehensile postures, and has been statistically validated with various subjects and artificial hand models. Using this protocol and the ABA, different prosthetic hands designed for 3D printing have been compared and, the effect of the subject on the grasping ability and the actuation synergies of the different models have been analyzed.

Grasping experiments with the human hand have been performed on twenty subjects and with a variety of objects in order to analyze the relative relevance of each of the different groups of degrees of freedom of the human hand in the main grasp types for autonomy during daily life. This information has been used to define an analytical index (Anthropomorphism Index of Mobility, AIM) that allows a straightforward evaluation of the mobility of 
an artificial hand based on the topology of the whole hand, joints and degrees of freedom, and the possibility to control them independently.

In addition, three anthropomorphism indexes have been proposed to compare the kinematic chain of an artificial hand with that of the human hand (Anthropomorphism Indexes of the Kinematic Chain, AIKCs), based on simplified hand models. The indexes defined use the basic parameters that define the kinematic chain, as well as other derived parameters: the reachable workspace by the different joints or the grasping postures adopted on various typical objects. Using the same simplified models of the kinematic chain, a method for the optimization of the thumb kinematic chain has been proposed from the simulation of the Kapandji test, commonly used for the functional evaluation of human hands, and it has been applied to the IMMA hand.

Finally, a preliminary method has been proposed to address the comparison of artificial hand design alternatives within the OpenRAVE grasping simulation environment by adapting the experimental AHAP. This method has been applied to evaluate and compare different design configurations of the IMMA hand. 


\section{RESUM}

L'objectiu d'aquesta tesi és el de contribuir a la definició de mètodes aplicables durant el procés de disseny de mans artificials per tal d'obtenir dissenys més antropomorfs i funcionals, així com definir mètriques i protocols que ajuden a avaluar aquests aspectes. Els mètodes proposats estan enfocats al disseny mecànic de qualsevol mà antropomorfa, tant en l'àmbit protèsic com en el robòtic, si bé en aquesta tesi s'ha fet un major èmfasi en mans orientades a la fabricació mitjançant impressió 3D, donat el seu creixent auge i accessibilitat i pel seu potencial impacte en la societat. Gran part dels desenvolupaments i propostes realitzades estan basats en aspectes biomecànics obtinguts de l'anàlisi de la mà humana. En aquesta tesi l'avaluació del grau d'antropomorfisme de les mans artificials s'ha abordat des de tres perspectives complementàries: experimentació, definició d'índexs analítics i simulació mitjançant models.

En primer lloc, s'ha dissenyat una mà protèsica (IMMA hand) actuada per sis cables/tendons independents i s'ha construït un primer prototip de la mateixa utilitzant impressió 3D. Per a l'avaluació experimental d'aquesta mà, o d'altres actuades per cable, per part de subjectes sans, s'ha dissenyat un dispositiu (able-bodied adaptor, ABA) que permet l'actuació dels tendons mitjançant els dits del subjecte alhora que es registra l'excursió en cada un dels tendons, habilitant l'anàlisi posterior de sinergies d'actuació, informació d'interès per al disseny dels sistemes d'actuació i/o control de les mans.

S'ha definit un protocol experimental, denominat Anthropomorphic Hand Assessment Protocol (AHAP), per a l'avaluació de l'antropomorfisme i funcionalitat de prototips de mans artificials. Aquest protocol inclou els vuit tipus d'agarrada més emprats per la mà humana, a part de dues postures no prènsils, i ha estat validat estadísticament amb diversos subjectes i models de mà artificial. Utilitzant aquest protocol i el ABA s'han comparat diferents mans protèsiques dissenyades per a impressió 3D i s'ha analitzat l'efecte del subjecte a l'avaluació de la capacitat prènsil i les sinergies d'actuació dels diferents models.

S'han realitzat experiments d'agarrada amb la mà humana sobre vint subjectes i amb una varietat d'objectes per tal d'analitzar la importància relativa de cada un dels diferents grups de graus de llibertat de la mà humana en les principals agarrades per a l'autonomia en la vida diària. Aquesta informació s'ha emprat per definir un índex analític (Anthropomorphism Index of Mobility, AIM) que permet una avaluació senzilla de la mobilitat 
d'una mà artificial en funció de la seua topologia, articulacions i graus de llibertat, i la possibilitat de controlar-los de manera independent.

A més, s'han proposat tres índexs de antropomorfisme que permeten comparar la cadena cinemàtica d'una mà artificial amb la de la mà humana (Anthropomorphism Indexes of the Kinematic Chain, AIKCs), basant-se en models simplificats d'aquestes. Els índexs definits utilitzen els paràmetres bàsics que defineixen la cadena cinemàtica, així com altres paràmetres derivats: l'espai de posicions assolibles per les diferents articulacions o les postures d'agarrada adoptades sobre diversos objectes típics. Utilitzant els mateixos models simplificats de la cadena cinemàtica s'ha proposat un mètode per a l'optimització de la cadena cinemàtica del polze a partir de la simulació del test de Kapandji, utilitzat per a l'avaluació funcional de mans humanes, i s'ha aplicat a la mà IMMA .

Finalment, s'ha realitzat una proposta preliminar d'un mètode per abordar la comparació d'alternatives de disseny de mans artificials dins de l'entorn de simulació d'agarrada OpenRAVE adaptant el protocol experimental AHAP. Aquest mètode s'ha aplicat per avaluar i comparar diferents configuracions de disseny de la mà IMMA. 


\section{RESUMEN}

El objetivo de esta tesis es el de contribuir a la definición de métodos aplicables durante el proceso de diseño de manos artificiales con el fin de obtener diseños más antropomorfos y funcionales, así como definir métricas y protocolos que ayuden a evaluar estos aspectos. Los métodos propuestos están enfocados al diseño mecánico de cualquier mano antropomorfa, tanto en el ámbito protésico como en el robótico, si bien en esta tesis se ha hecho un mayor énfasis en manos orientadas a la fabricación mediante impresión $3 \mathrm{D}$, dado su creciente auge y accesibilidad y por su potencial impacto en la sociedad. Gran parte de los desarrollos y propuestas realizados están basados en aspectos biomecánicos obtenidos del análisis de la mano humana. En esta tesis la evaluación del grado de antropomorfismo de las manos artificiales se ha abordado desde tres perspectivas complementarias: experimentación, definición de índices analíticos y simulación mediante modelos.

En primer lugar, se ha diseñado una mano protésica (IMMA hand) actuada por seis cables/tendones independientes y se ha construido un primer prototipo de la misma utilizando impresión 3D. Para la evaluación experimental de dicha mano, u otras actuadas por cable, por parte de sujetos sanos, se ha diseñado un dispositivo (able-bodied adaptor, ABA) que permite la actuación de los tendones mediante los dedos del sujeto a la vez que se registra la excursión en cada uno de los tendones, habilitando el posterior análisis de sinergias de actuación, información de interés para el diseño de los sistemas de actuación y/o control de las manos.

Se ha definido un protocolo experimental, denominado Anthropomorphic Hand Assessment Protocol (AHAP), para la evaluación del antropomorfismo y funcionalidad de prototipos de manos artificiales. Este protocolo incluye los ocho tipos de agarre más empleados por la mano humana, aparte de dos posturas no prensiles, $\mathrm{y}$ ha sido validado estadísticamente con diversos sujetos y modelos de mano artificial. Utilizando dicho protocolo y el ABA se han comparado diferentes manos protésicas diseñadas para impresión 3D y se ha analizado el efecto del sujeto en la evaluación de la capacidad prensil y las sinergias de actuación de los diferentes modelos.

Se han realizado experimentos de agarre con la mano humana sobre veinte sujetos y con una variedad de objetos con el fin de analizar la importancia relativa de cada uno de los diferentes grupos de grados de libertad de la mano humana en los principales agarres para la autonomía en la vida diaria. Dicha información se ha empleado para definir un índice 
analítico (Anthropomorphism Index of Mobility, AIM) que permite una evaluación sencilla de la movilidad de una mano artificial en función de su topología, articulaciones y grados de libertad, y la posibilidad de controlarlos de forma independiente.

Además, se han propuesto tres índices de antropomorfismo que permiten comparar la cadena cinemática de una mano artificial con la de la mano humana (Anthropomorphism Indexes of the Kinematic Chain, AIKCs), en base a modelos simplificados de las mismas. Los índices definidos utilizan los parámetros básicos que definen la cadena cinemática, así como otros parámetros derivados: el espacio de posiciones alcanzables por las diferentes articulaciones o las posturas de agarre adoptadas sobre diversos objetos típicos. Utilizando los mismos modelos simplificados de la cadena cinemática se ha propuesto un método para la optimización de la cadena cinemática del pulgar a partir de la simulación del test de Kapandji, utilizado para la evaluación funcional de manos humanas, y se ha aplicado a la mano IMMA.

Finalmente, se ha realizado una propuesta preliminar de un método para abordar la comparación de alternativas de diseño de manos artificiales dentro del entorno de simulación de agarre OpenRAVE adaptando el protocolo experimental AHAP. Dicho método se ha aplicado para evaluar y comparar diferentes configuraciones de diseño de la mano IMMA. 


\section{AGRADECIMIENTOS ACKNOWLEDGEMENTS}

Quiero empezar agradeciendo especialmente a mi director de tesis, el Dr. Antonio Pérez, por enseñarme y guiarme en todo momento, por su confianza depositada y el tiempo invertido en mí, por su paciencia infinita, y por haberme acompañado en todo este camino contribuyendo en mi crecimiento personal y profesional. Además de todo lo que me ha enseñado en estos años de carrera académica, docente e investigadora, también me quedo con todos los momentos compartidos, desde el primer Skype estando yo de Erasmus en Bruselas, pasando por todos los congresos en los diferentes lugares del mundo, evaluando mis primeras clases como docente, los días enteros metida en su despacho discutiendo correcciones, e incluso en mi boda. Momentos en los que nunca ha faltado la risa como fiel aliada. Ha sido mi mayor apoyo, con quien más tiempo y experiencias he compartido durante estos intensos años, todo un referente para mí en todos los sentidos.

En segundo lugar, agradecer a todos los miembros del grupo de investigación su apoyo y colaboración. En especial quiero mencionar a Marta Mora y José Vicente García por los debates y consejos durante las comidas, a Javier Andrés, Pepe Iserte y Verónica Gracia por confiar en mí y ayudarme a llevar a cabo mis ideas, a Pepe Fuentes por sus consejos y por ayudarme a fabricar las diferentes versiones de mis diseños, a Ximo Sancho por su valoración y motivación, y a los estudiantes Jesús Cantero y César Corella por su dedicación. También agradecer al resto de compañeros del departamento sus consejos, su apoyo y motivación. En especial a Fran Sánchez por su colaboración en mi propuesta de mejora educativa, y a él y Víctor Roda por su paciencia en mis primeros años como docente. También a Antonio Morales y Carlos Rubert del Robotic Intelligence Lab por su colaboración en los proyectos de investigación.

I would also like to thank Prof. Tamim Asfour for his collaboration, support and warm welcome I received during the four months I was in his laboratory at the KIT. I want to express my great thanks to Julia Starke and Júlia Borràs for taking care of me, for always offering me the help I needed and for the research collaboration. I met wonderful people like Pascal Weiner, Jonas Beil, Isabel Patzer, Samuel Rader, etc. I hope I see you all again very soon.

Y por último, pero no por ello menos importante, quiero agradecer el apoyo incondicional y motivación constante de mi familia y amigos, 
especialmente de mis padres y mi marido. A Jesús e Inma, porque todo lo que soy es gracias a vosotros. Porque sé lo orgullosos que estáis de mí por haber llegado hasta aquí, pero todo es gracias a vuestro apoyo y consejos. A mi hermana pequeña, Sílvia, porque mi superación se basa en ser un ejemplo para ti. Y a ti, Miguel, por todo el cariño y apoyo que me das cada día, por comprenderme y sacarme siempre una sonrisa. Por animarme a hacer todo aquello que me hace feliz, por ayudarme a conseguir todo lo que me propongo, por estar siempre a mi lado y recordarme de lo que soy capaz. También a mis amigos Sandra, Pedro, Alba, Javi... por estar siempre a mi lado a pesar de la distancia. Y a mis bailarinas favoritas por aportarme el combustible que necesitaba durante la recta final. 


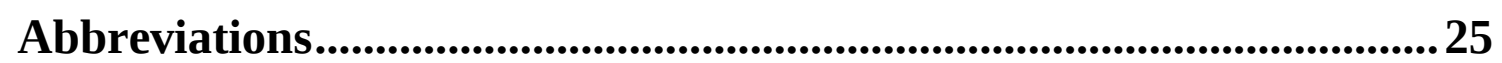

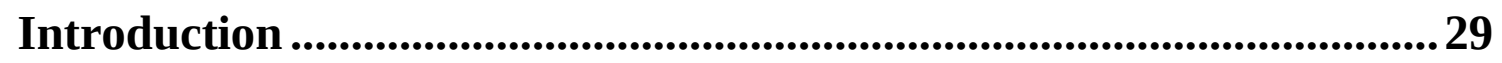

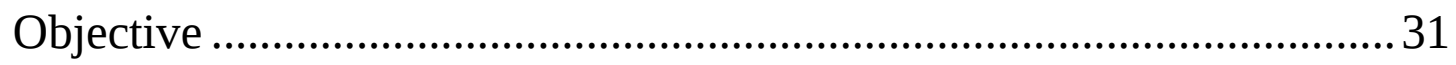

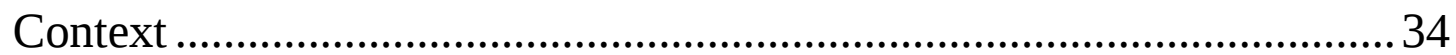

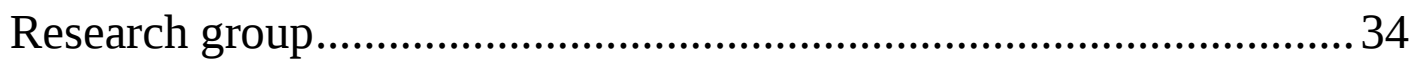

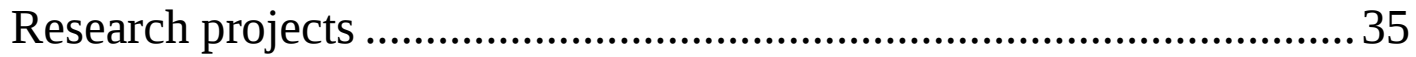

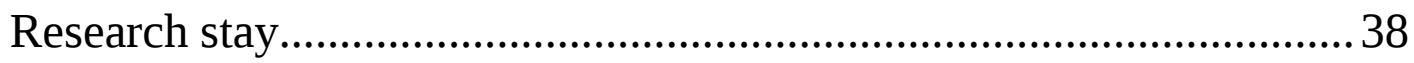

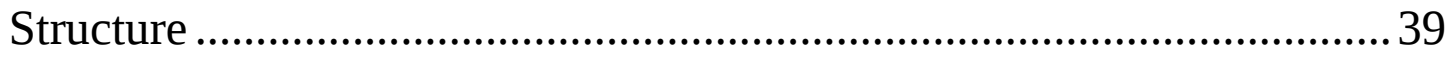

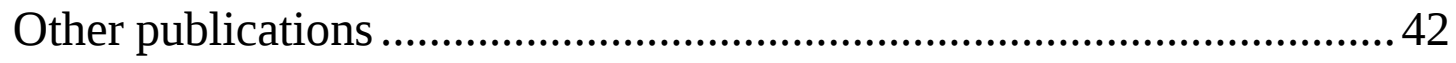

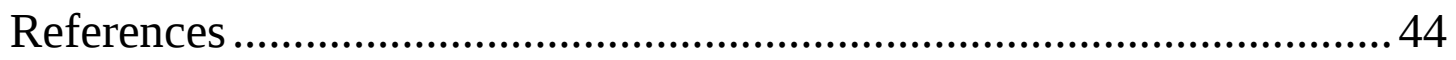

Chapter 1. System for the experimental evaluation of anthropomorphic hands. Application to a new 3D-printed prosthetic hand prototype................................................................................................... 49

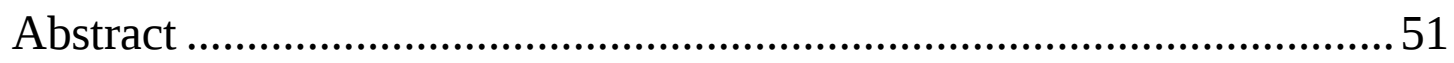

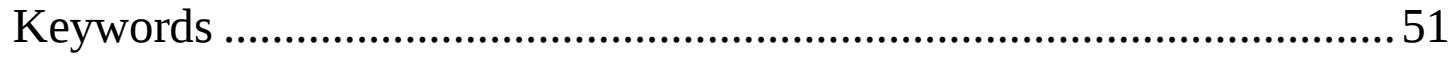

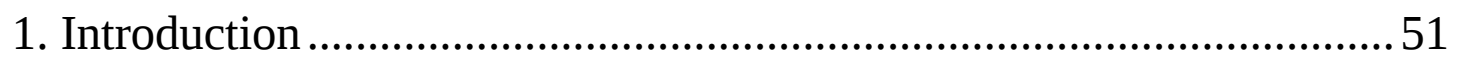

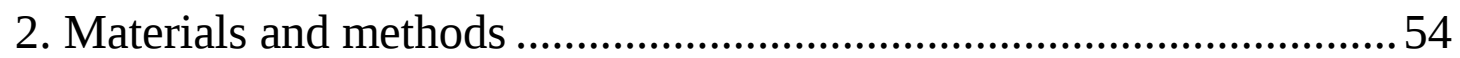

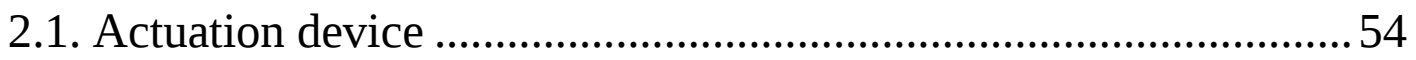

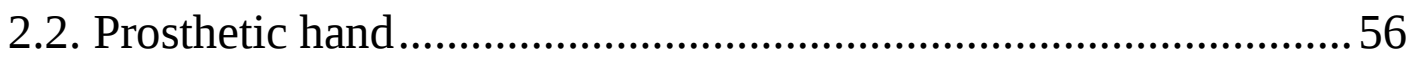

2.3. Protocol for testing hand prototypes ................................................ 59

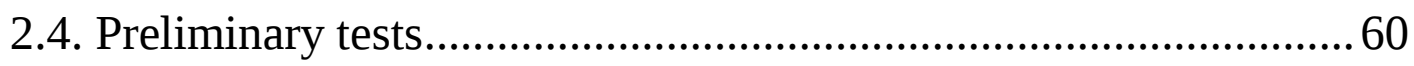

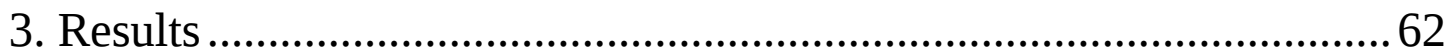

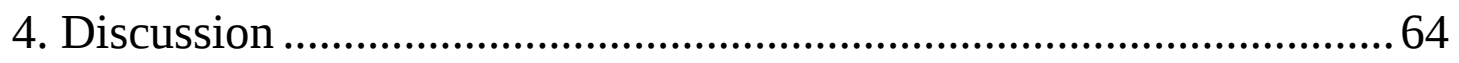

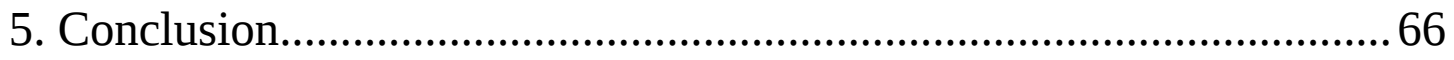

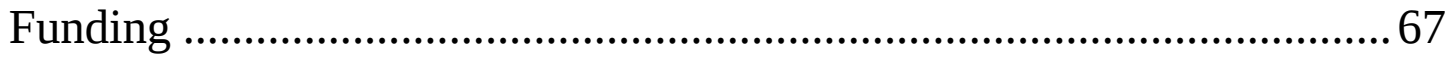

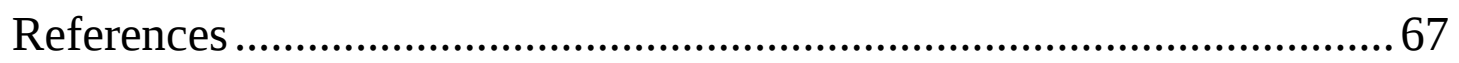

Chapter 2. The Anthropomorphic Hand Assessment Protocol (AHAP) 71

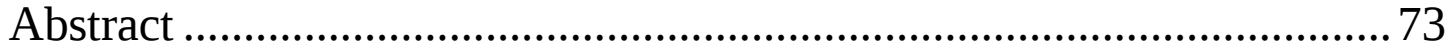




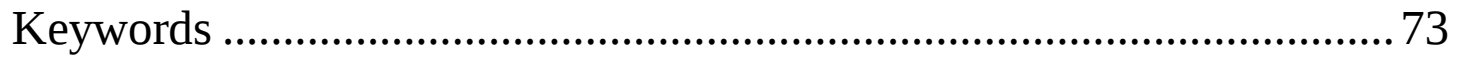

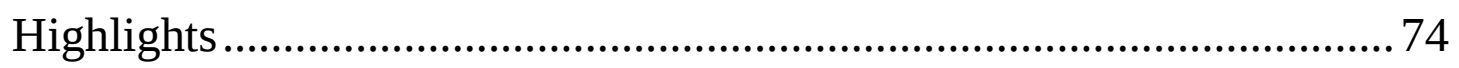

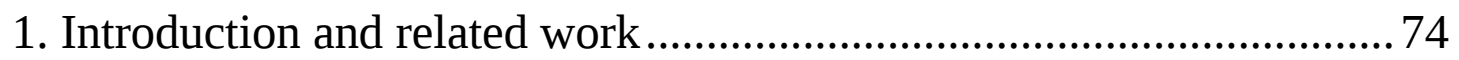

2. The Anthropomorphic Hand Assessment Protocol (AHAP)................ 77

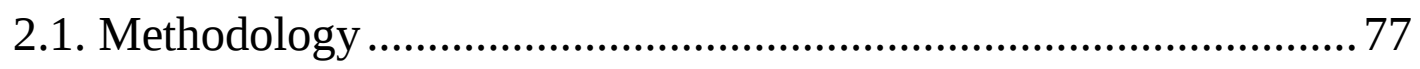

2.2. Grasp types and objects ................................................................. 79

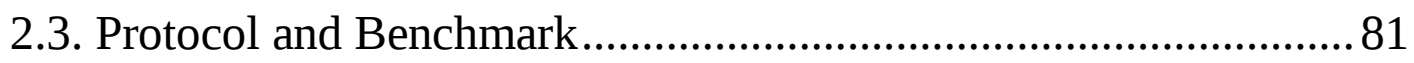

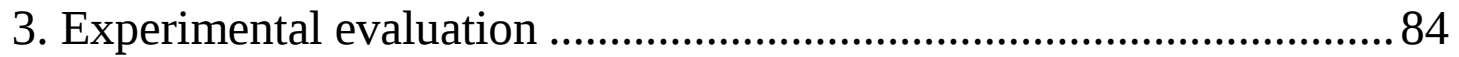

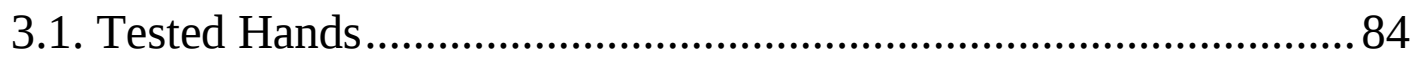

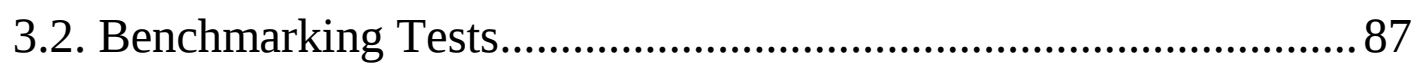

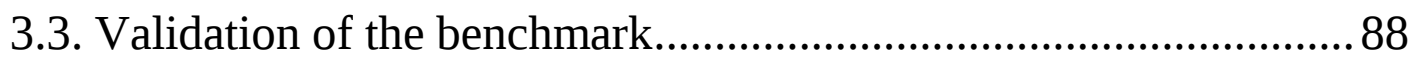

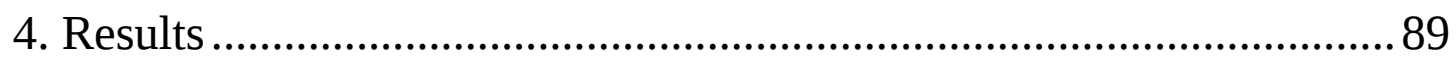

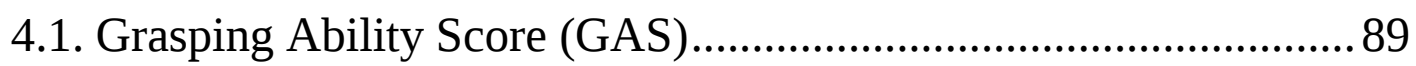

4.2. Validation of the benchmark...................................................... 91

4.3. Qualitative impressions from the grasp trials ................................ 92

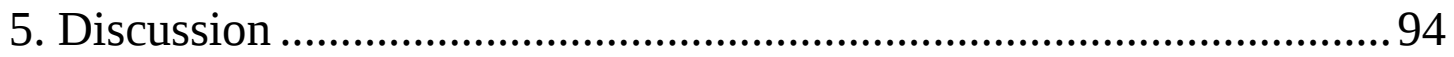

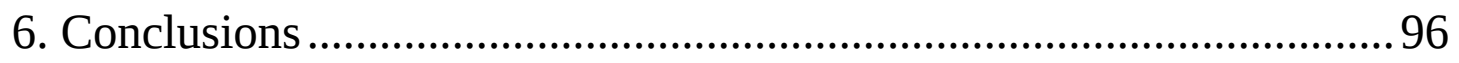

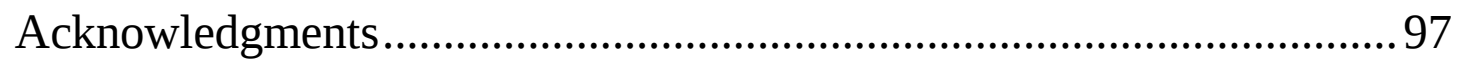

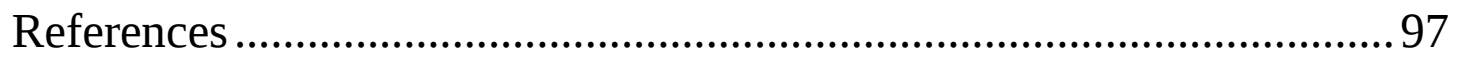

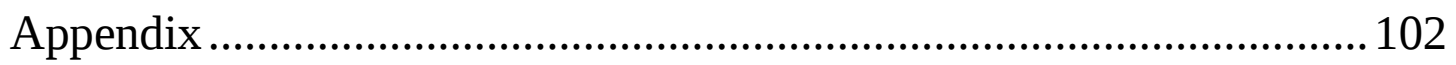

Chapter 3. Grasping ability and motion synergies in affordable tendon-driven prosthetic hands controlled by able-bodied subjects.. 103

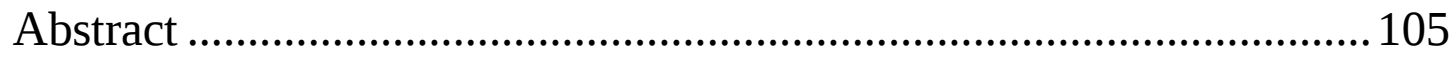

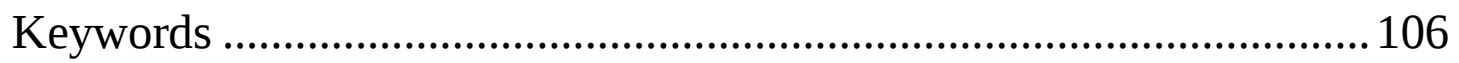

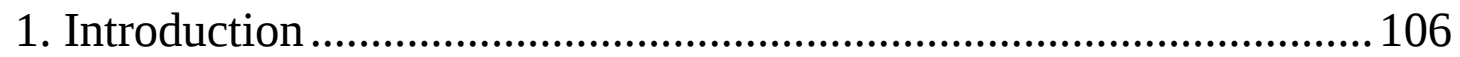

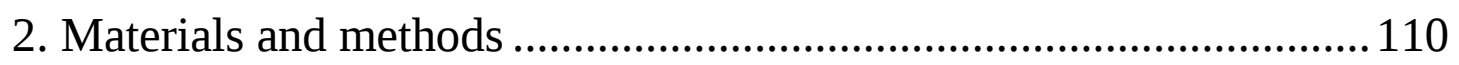

2.1. Tendon-driven prosthetic hands................................................ 110

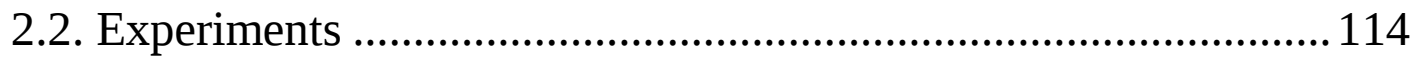

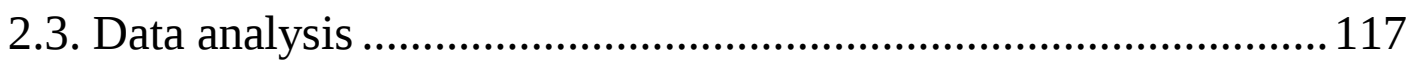

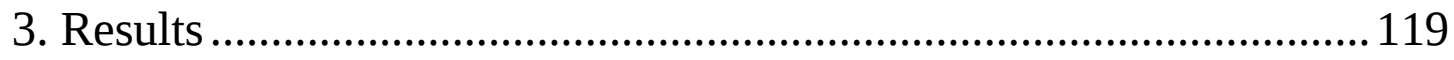

3.1. Grasping Ability Score (GAS)................................................... 119 


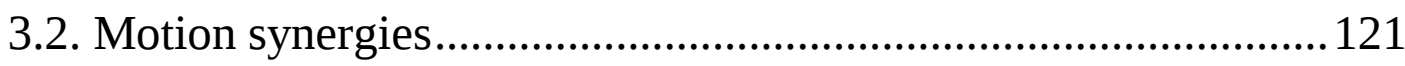

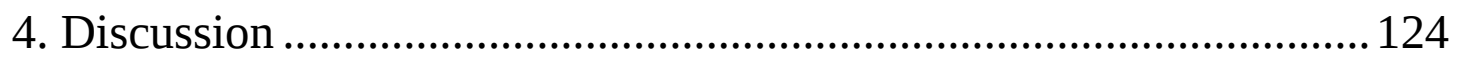

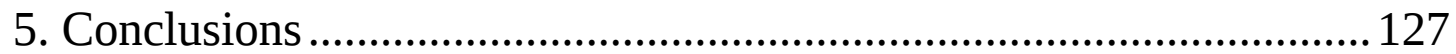

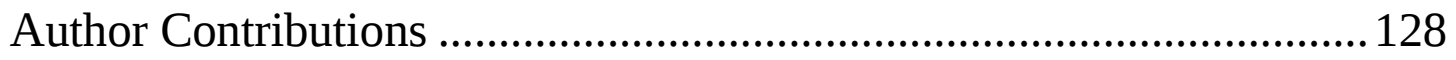

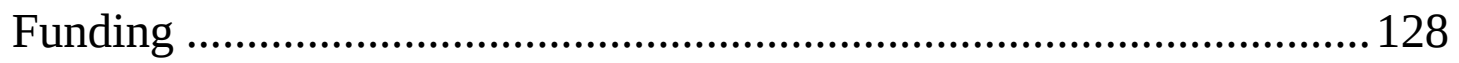

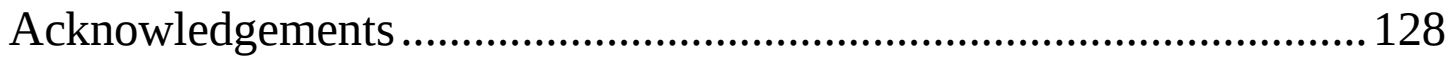

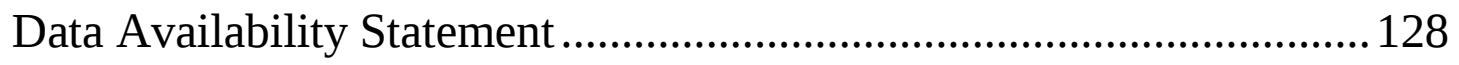

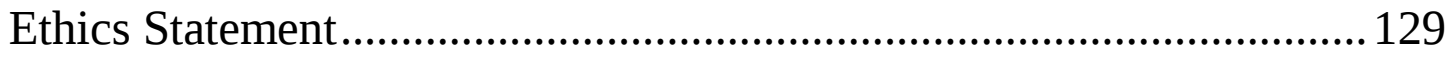

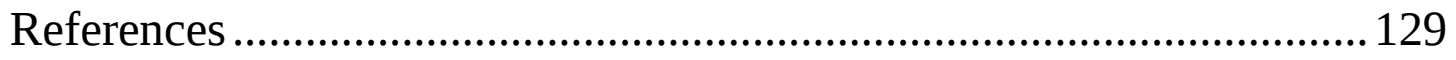

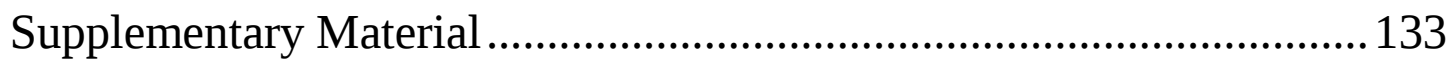

\section{Chapter 4. Anthropomorphism Index of Mobility for Artificial Hands}

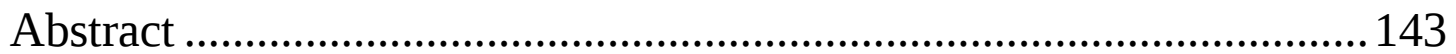

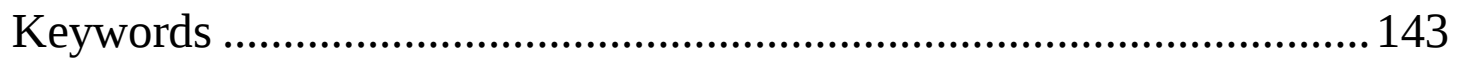

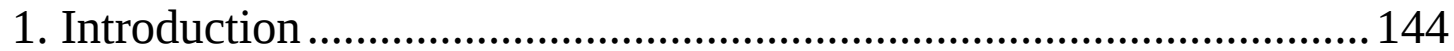

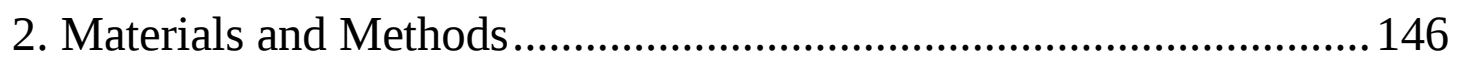

2.1. Human Grasp Experiment .................................................... 146

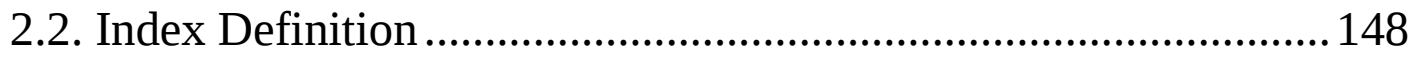

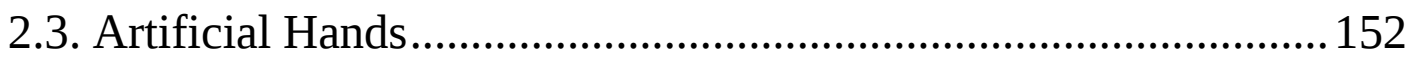

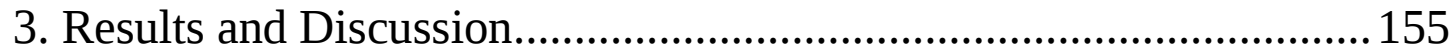

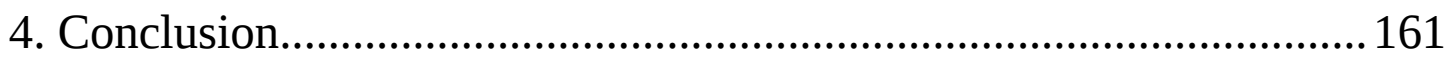

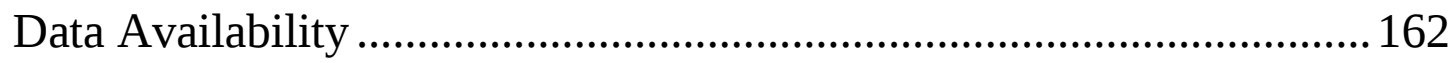

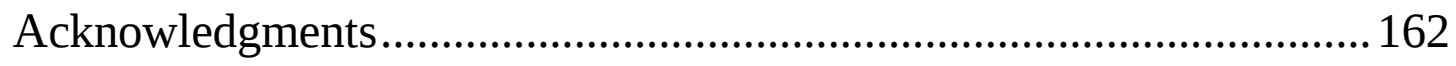

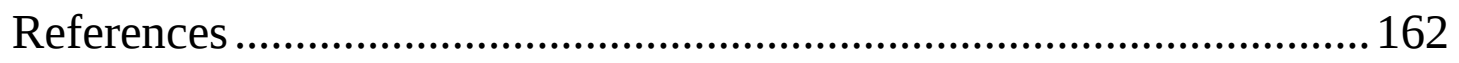

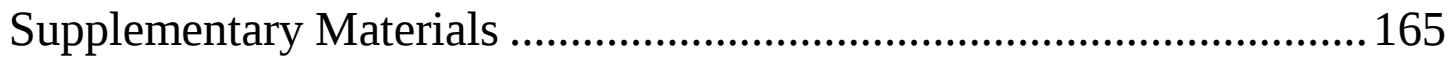

Chapter 5. Anthropomorphism Indexes of the Kinematic Chain for Artificial Hands ................................................................................167

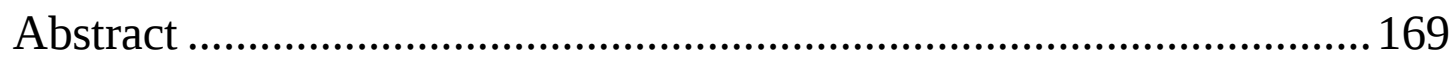

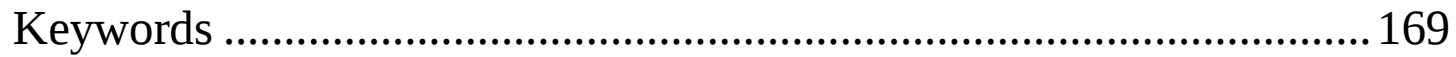

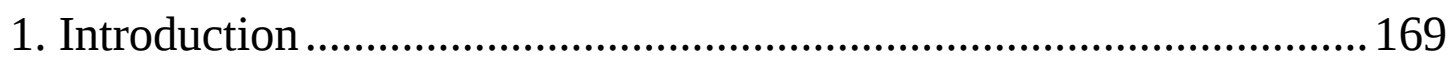

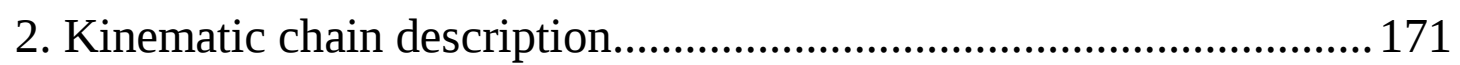


2.1. Kinematic chain of the human hand ........................................... 171

2.2. Definition of the kinematic chain ................................................ 173

3. Anthropomorphism index of the kinematic chain (AIKC) ................ 175

3.1. AIKC $C_{1}$ : based on the parameters of the kinematic chain .............. 175

3.2. $A I K C_{2}$ : based on the workspace.................................................. 177

3.3. $A I K C_{3}$ : based on the comparison of common grasping postures. 178

4. Evaluation of artificial hands .......................................................... 180

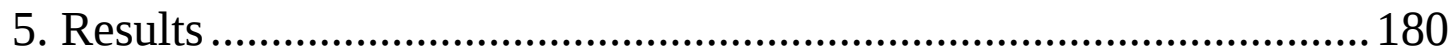

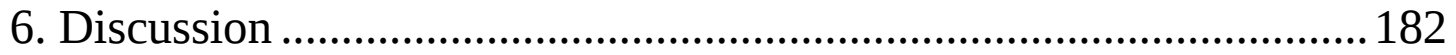

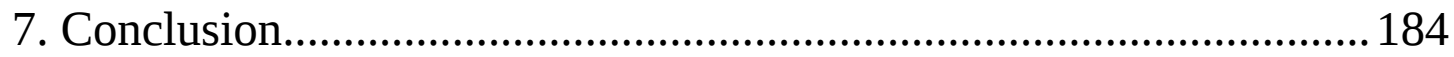

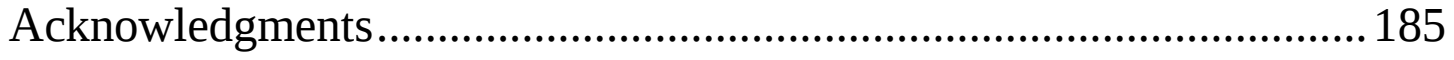

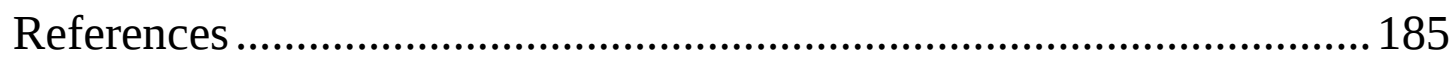

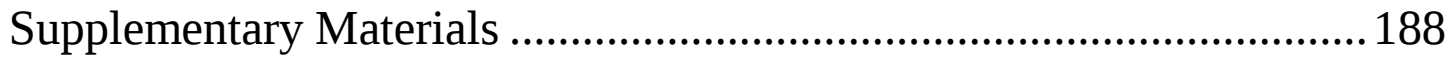

Chapter 6. Optimization of the Kinematic Chain of the Thumb for a Hand Prosthesis Based on the Kapandji Opposition Test .................... 197

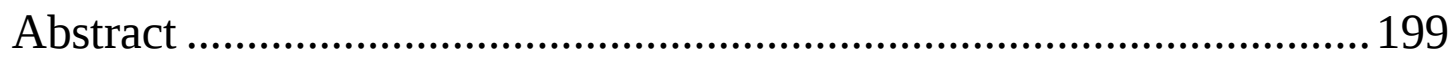

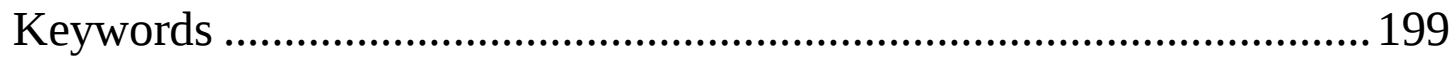

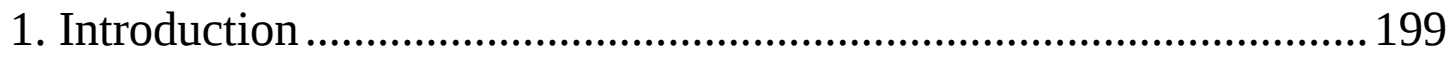

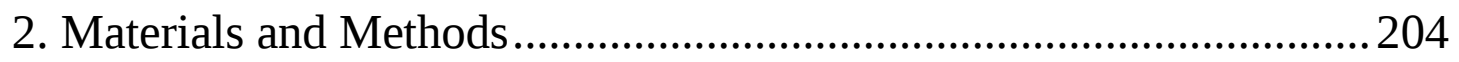

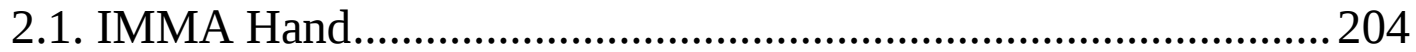

2.2. Computational Model .................................................................. 205

2.3. Case study: Optimization of the TKC of the IMMA hand .......... 208

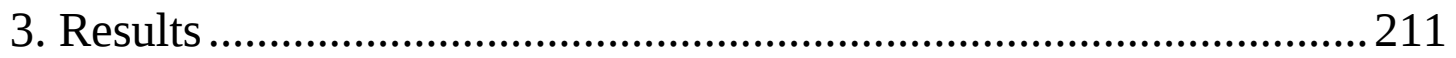

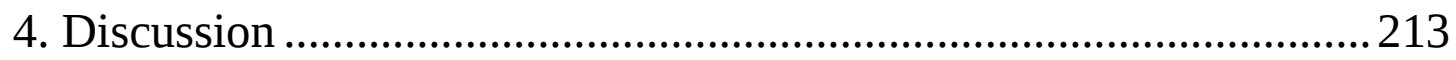

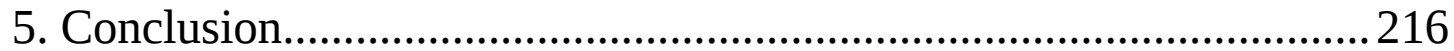

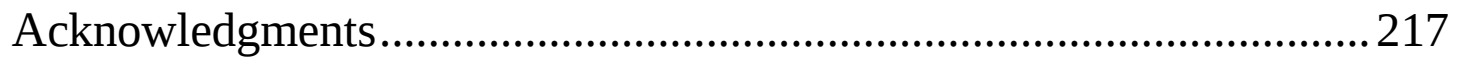

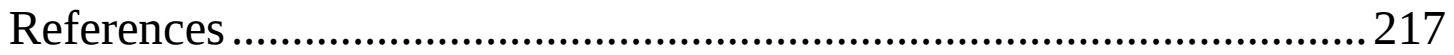

Chapter 7. Benchmarking prosthetic hands through anthropomorphic grasping simulations ........................................................................................ 219

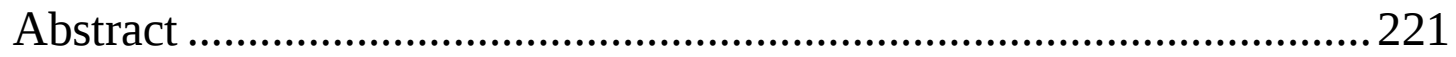

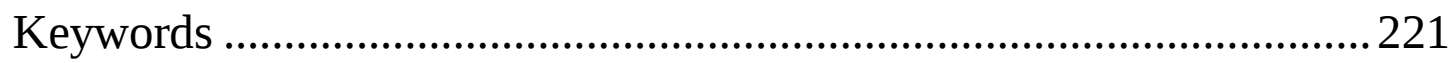




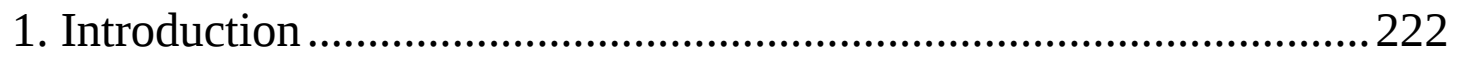

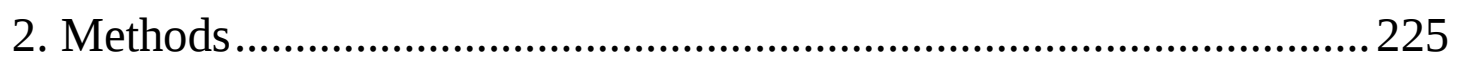

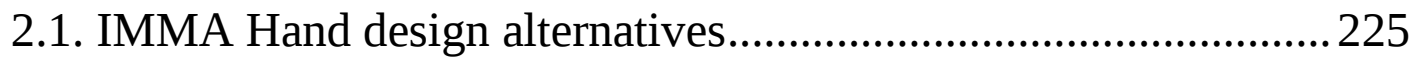

2.2. Grasp types and objects ................................................................ 226

2.3. Grasp simulation: brute-force approach ..................................... 227

2.4. Grasp simulation: Anthropomorphic grasps approach ................. 229

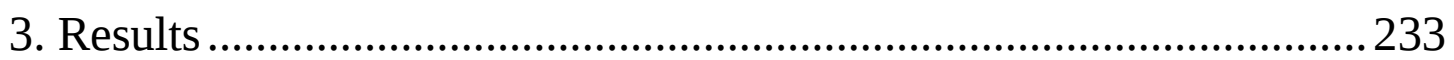

3.1. Brute-force approach ................................................................. 233

3.2. Anthropomorphic grasps approach .............................................. 234

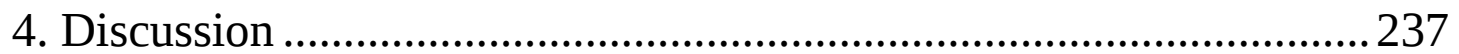

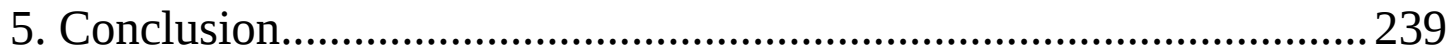

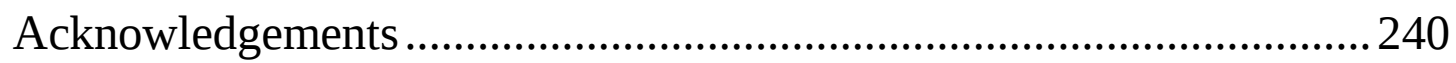

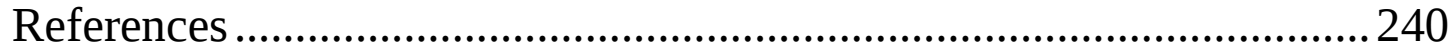

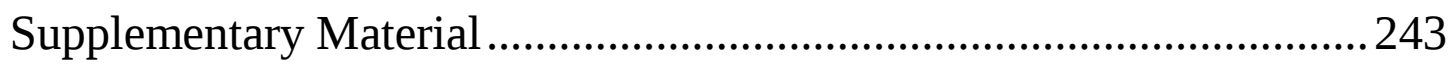

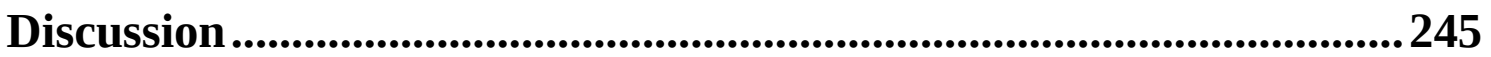

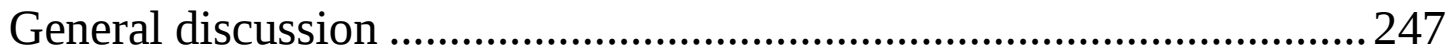

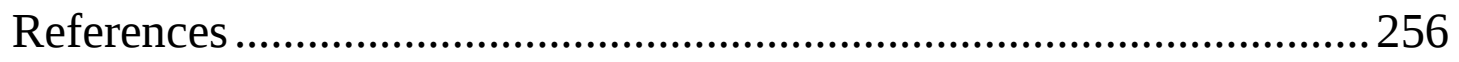

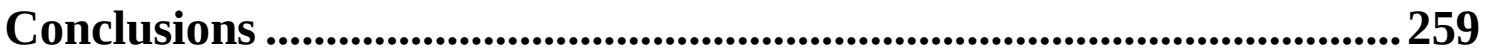

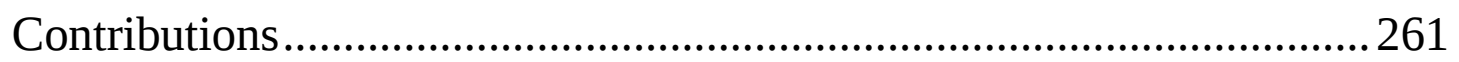

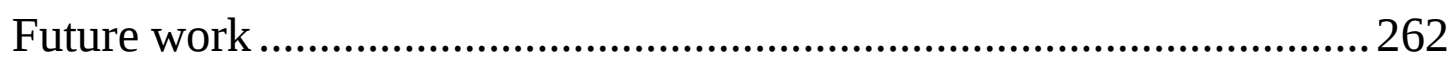



Abbreviations 

3D: $\quad$ Three-dimensional

AB/AD: Abduction/adduction

ABA: Able-bodied adaptor

ABS: $\quad$ Acrylonitrile butadiene styrene

ADL: Activity/ies of daily living

AH: $\quad$ Artificial hand

AHA: Assisting Hand Assessment

AHAP: Anthropomorphic Hand Assessment Protocol

AI: $\quad$ Anthropomorphism Index

AIKC: $\quad$ Anthropomorphism Index of the Kinematic Chain

AIM: $\quad$ Anthropomorphism Index of Mobility

ANOVA: Analysis of variance

BBT: $\quad$ Box and Block Test

CAD: $\quad$ Computer-Aided Design

CC: $\quad$ Correlation Coefficient

CG: Cylindrical grip

CMC: Carpometacarpal

DA: Design alternative

DH: Denavit-Hartenberg

DIP: $\quad$ Distal interphalangeal

DIY: $\quad$ Do-it-yourself

DoA: Degree of actuation

DoF: $\quad$ Degree of freedom

DP: $\quad$ Distal phalanx

DVG: Diagonal volar grip

EG: $\quad$ Extension grip

F/E: $\quad$ Flexion/extension

FDM: $\quad$ Fused deposition modeling

GAS: $\quad$ Grasping Ability Score

GH: $\quad$ Grasp hypothesis

GQM: $\quad$ Grasp quality metrics

GT: $\quad$ Grasp type

H: $\quad$ Hook

$\mathrm{H}^{2} \mathrm{~T}$ : $\quad$ High Performance Humanoid Technologies Lab

HH: Human hand

ICC: Intraclass correlation coefficient 


\section{8 | Abbreviations}

ICF: $\quad$ International Classification of Functioning, Disability and Health

IP: $\quad$ Index pointing/pressing or Interphalangeal

KC: $\quad$ Kinematic chain

KIT: $\quad$ Karlsruhe Institute of Technology

KOT: $\quad$ Kapandji opposition test

LCS: $\quad$ Local coordinate system

LP: $\quad$ Lateral pinch

MC: $\quad$ Metacarpal

MCP: $\quad$ Metacarpophalangeal

MP: $\quad$ Medial phalanx

MPE: $\quad$ Mean position error

NHPT: $\quad$ Nine Hole Peg Test

NIST: $\quad$ National Institute of Standards and Technology

P: $\quad$ Platform

P.ARC: Palmar arching

PC: $\quad$ Principal component

PCA: $\quad$ Principal Component Analysis

PIP: $\quad$ Proximal interphalangeal

PLA: $\quad$ Polylactic acid

PP: $\quad$ Pulp pinch or Proximal phalanx

ROM: $\quad$ Range of motion

RP: $\quad$ Reference posture

SG: $\quad$ Spherical grip

SGAS: $\quad$ Simulated Grasping Ability Score

SHAP: $\quad$ Southampton Hand Assessment Procedure

SRM: $\quad$ Standardized response mean

T.OPP: Thumb opposition

TDPH: $\quad$ Tendon-driven prosthetic hand

TKC: $\quad$ Thumb's kinematic chain

TP: $\quad$ Tripod pinch

TPS: $\quad$ Tendon pair synergy

TPU: $\quad$ Thermoplastic polyurethane

UJI: $\quad$ Universitat Jaume I

YCB: $\quad$ Yale-CMU-Berkeley 


\section{Introduction}





\section{Objective}

Recently there has been a significant progress in the development of anthropomorphic artificial hands both for robotic applications such as humanoid robotics and human-robot cooperation (Controzzi et al., 2014; Puig et al., 2008) and for prosthetic hands (Belter et al., 2013). Additionally, 3D printing technologies have facilitated the advancement of affordable prosthetic hands (ten Kate et al., 2017) enabling customization and selfmanufacture under the premise of do-it-yourself (DIY). Initiatives such as eNABLE (enablingthefuture.org), Open Hand Project (openhandproject.org), or Open Bionics (openbionics.com) are the main source for CAD repositories where anyone can freely download a hand model and print it. However, the human hand is a complex and marvelous tool whose dexterity has not yet been achieved by any artificial hand. According to several studies (Duong et al., 2017; Li et al., 2019; OpenAI et al., 2018; Simon et al., 2019) its degree of functionality is far from being achieved even by expensive commercial prostheses or sophisticated robotic hands.

Comparing the grasping ability and functionality of artificial hands with those of the human hand is essential for improving current anthropomorphic hand designs. Notwithstanding, the progress in the development of anthropomorphic hands has not been followed by a parallel development of objective methods to evaluate or compare the performance of different hand designs. The need for benchmarking in this field has been recognized by several standardization organizations as the NIST (Falco et al., 2015) and different researchers (Bonsignorio et al., 2014; Calli et al., 2015; Mio et al., 2018; Quispe et al., 2018). In general, standardized performance testing or benchmarking is a fundamental tool that is crucial for the progress of any activity of research and development. It provides the ability to replicate and compare quantified results to enhance understanding of the effectiveness of an approach for improving product designs. Currently, however, the design of anthropomorphic hands is not generally based on grasp metrics or standard experimental protocols. Orthopedic companies base their designs mainly on previous experience and user feedback, with an emphasis on grip modes and aesthetics.

Therefore, the main objective of this thesis is to develop analytical and experimental methods to evaluate anthropomorphic artificial hands. These methods are intended to be useful to analyze their limitations and to optimize their designs. 


\section{2 | Introduction}

With this focus, it is important to note that anthropomorphism can be understood as similarity to the human hand in terms of size, weight, shape, appearance, temperature, etc., i.e. cosmesis (ten Kate et al., 2017), or as similarity in terms of functionality or dexterity (Liarokapis et al., 2012). Functional aspects are clearly more complex in terms of evaluation. The final functionality is affected by the mechanical design of the hand (kinematic chain, materials, transmissions), but also by the actuation method (actuators performance, underactuation degree) and by the control system (brainmachine interfaces, sensors, feedback, control design). This thesis is based on the hypothesis that it is helpful to isolate these different aspects for a better understanding of their effect on the functionality. Assessing the capability of artificial hands to perform the main grasp types (GTs) of human grasping in activities of daily living (ADL) could give an insight into their level of functionality. Moreover, the architecture of the kinematic chain of the artificial hand is of primary importance to achieve anthropomorphism in both senses, cosmesis and functionality, so it should be considered in the comparison of hand designs.

The complexity of the anthropomorphic hands challenges the design of useful comparison indexes and grasping performance benchmarks. Several approaches can be considered: experimentation, analytical indexes or grasping simulation.

Experimental approaches allow assessing the grasping ability of artificial hands with realistic information about the final performance. However, experimental tests and protocols for overall evaluation or comparison of different mechanical designs in terms of their anthropomorphism or grasping ability are scarce in the scientific literature. Besides that, experimental approaches are costly and time-consuming and require the use of physical prototypes, which is inconvenient in early design stages.

Alternatively, the comparison of the hand features with those of the human hand through simplified models or computer simulation can be useful to evaluate and rank design alternatives. Some studies (Feix et al., 2013a; Liarokapis et al., 2013) proposed anthropomorphism indexes based on the comparison of the workspace of the artificial hand and that of the human hand, although they include some limitations in their definition. Others (Biagiotti et al., 2003; Jiang et al., 2018; Liu et al., 2015) tried to define simple analytical indexes to compare artificial hand designs, but the comparison with the human hand is made on general features instead of its real grasping ability. 
Finally, grasping simulation has been tried in the literature but its complexity for anthropomorphic hands is a limitation, making it challenging to obtain a good representation of the real world situation. Many studies in the literature about grasp planning used grasp simulation and defined different grasp quality metrics (GQM) with the aim of quantifying the grasp quality of a given object-hand-posture set, but there is not consensus about which is the best GQM. The use of combined metrics has been suggested as a method to find a more robust estimator of the grasp quality, although an optimal solution has not been found (Roa and Suárez, 2014). Some attempts have been made in order to use this approach to compare artificial hand designs (León et al., 2013, 2012; Rubert et al., 2017; Rubert and Morales, 2016).

Given the complexity to get an overall solution to the main objective of this thesis, and based on the hypothesis that it is interesting to split the different design aspects in order to better understand their effect on the hand functionality, I have limited the scope, excluding the control system of the artificial hands in the methods and indexes proposed. Oppositely, the mechanical design and the actuation methods are considered in most of the methods proposed. Additionally, and without loss of generality in the proposed methods, I have paid particular attention to affordable 3D-printed tendon-driven prosthetic hands (TDPHs) because, as cited above they are a rising trend due to their availability and easy customization.

Considering all above, the main objective of this thesis has been split into the following specific objectives during the different stages of the thesis:

1. Design of a new TDPH with six actuated degrees of freedom (DoFs) and manufacture of a 3D-printed prototype, which could be assessed experimentally and analytically.

2. Definition of a universal experimental benchmark to evaluate the ability of both robotic and prosthetic anthropomorphic hands to produce successful grasps in a human-like manner. The standardized protocol should be a functional test including the main GTs typical of human grasping in ADL.

3. Design of an able-bodied adaptor (ABA) that can be adapted to the arm of a healthy subject enabling the actuation of up to six tendons of TDPHs with its own fingers while registering tendon excursions.

4. Analysis of the motion synergies during the actuation of TDPHs when performing the proposed experimental benchmark with the ablebodied adaptor (ABA). 


\section{4 | Introduction}

5. Comparison of the grasping ability of different anthropomorphic hands with the proposed experimental benchmark and analysis of the main limitations for performing the most common GTs.

6. Experimental analysis of the relevance of the different degrees of freedom (DoFs) of the human hand on the most important GTs.

7. Definition of an analytical index able to evaluate the anthropomorphism of artificial hands based on the mobility supplied by the actuation and transmission systems to the different DoFs according to their relevance observed in human grasping.

8. Proposal of new indexes to evaluate the anthropomorphism of the kinematic chain of artificial hands.

9. Comparison of different current artificial hands with the analytical indexes proposed.

10.Definition of a computational method to optimize the kinematic chain of the thumb of an artificial hand based on its opposition.

11.Proposal of a preliminary approach to a new benchmark for evaluating anthropomorphic artificial hands based on the adaptation of the experimental benchmark proposed in this thesis to an existing grasping simulation framework.

12.Comparison of several hand design alternatives for the TDPH designed in this thesis using the proposed grasping simulation benchmark.

\section{Context}

This thesis is framed within three research projects funded by public entities and a predoctoral contract related to one of them. The ideas for the thesis resulted from my research within the Biomechanics \& Ergonomics group at Universitat Jaume I (UJI) of which I am a member. Moreover, a research stay at the Karlsruhe Institute of Technology (KIT), in Germany, under the supervision of Dr. Prof. Tamim Asfour, also collaborating in the cited projects, led to part of the work presented here.

\section{Research group}

The main research lines undertaken by the Biomechanics \& Ergonomics group include: human hand biomechanics, hand tools ergonomics, foot biomechanics, knee biomechanics, dental biomechanics, and emotional design. The line of research about design and evaluation of anthropomorphic artificial hands arose from the perspective that the extensive background of 
the group in human hand biomechanics may be useful for obtaining better artificial hands. Previous works of the group can be considered as a large knowledge base applicable to anthropomorphic hands, among which are: stiffness of grasping contact areas of the human hand (Pérez-González et al., 2013), grip force and force sharing in manipulation tasks (Cepriá-Bernal et al., 2016), common grasps during ADL (Vergara et al., 2014), relevance of GTs to assess functionality for personal autonomy (Gracia-Ibáñez et al., 2018), functional range of motion of the hand joints (Gracia-Ibáñez et al., 2017b), dorsal and palmar aspect dimensions of the hand (Vergara et al., 2018), etc. The equipment of the research group laboratory allowed to develop methods to measure the hand movements with videogrammetry (Sancho-Bru et al., 2014) and instrumented gloves (Gracia-Ibáñez et al., 2017a) contributing to the hand functional assessment (Gracia Ibañez, 2016). In addition, modeling work within the group led to the development of a widely cited biomechanical model of the human hand (Sancho-Bru, 2000) which was applied to grasp simulations (Sancho-Bru et al., 2012, 2003). In collaboration with the Robotic Intelligence Lab at UJI this biomechanical model was introduced in grasping simulation tools (León et al., 2014) and its prehension was evaluated with the grasp quality measures used in robotics (León et al., 2012). Later, these metrics were also preliminary tested to evaluate artificial hands (Rubert et al., 2017). All this background of the research group in the field of robotics and grasping simulation is also very useful for application in the evaluation of anthropomorphic hands.

\section{Research projects}

This thesis is framed within three research projects (Table 1) funded by public entities in which I participated and a FPI-MINECO predoctoral contract funded by the Spanish Ministry of Economy and Competitiveness and ESF (grant number BES-2015-076005) and connected to one of the projects (grant number DPI2014-60635-R).

Table 1. Research projects.

\begin{tabular}{lll}
\hline 1 Acronym & DEVALHAND \\
Reference & DPI2014-60635-R \\
& Title & $\begin{array}{l}\text { Design and evaluation of anthropomorphic hands by } \\
\text { using grasping simulation. Application to the design } \\
\end{array}$ \\
& and control of prosthetic hands.
\end{tabular}


Institution

Period

Funding

Principal Investigators

Research team

Work team

Period

Funding

Principal Investigators

Research team

Reference

Title

Institution
Spanish Ministry of Economy and Competitiveness, AEI and ERDF

Jan. 2015 - Sep. 2018

$115000 €$

Antonio Pérez González

Antonio Morales Escrig

F. Javier Andrés de la Esperanza

Marta C. Mora Aguilar

José V. García Ortiz

Tamim Asfour (KIT)

Beatriz León Pinzón (Shadow Robots)

Pierre-Yves Joubert (University Paris Sud)

Emile Martincic (University Paris Sud)

Carlos Rubert Escuder

Juan Laforga Cocho (Shadow Robots)

Immaculada Llop Harillo

Higinio Martí Ribes
2 Acronym

Antonio Morales Escrig

F. Javier Andrés de la Esperanza

Marta C. Mora Aguilar

José V. García Ortiz 
José L. Iserte Vilar

Work team

Tamim Asfour (KIT)

Emile Martincic (University Paris Sud)

Arjan Buis (University of Strathclyde)

Sarah Day (University of Strathclyde)

Immaculada Llop Harillo

José F. Fuentes Ballesteros

Daniel Cardín Catalán

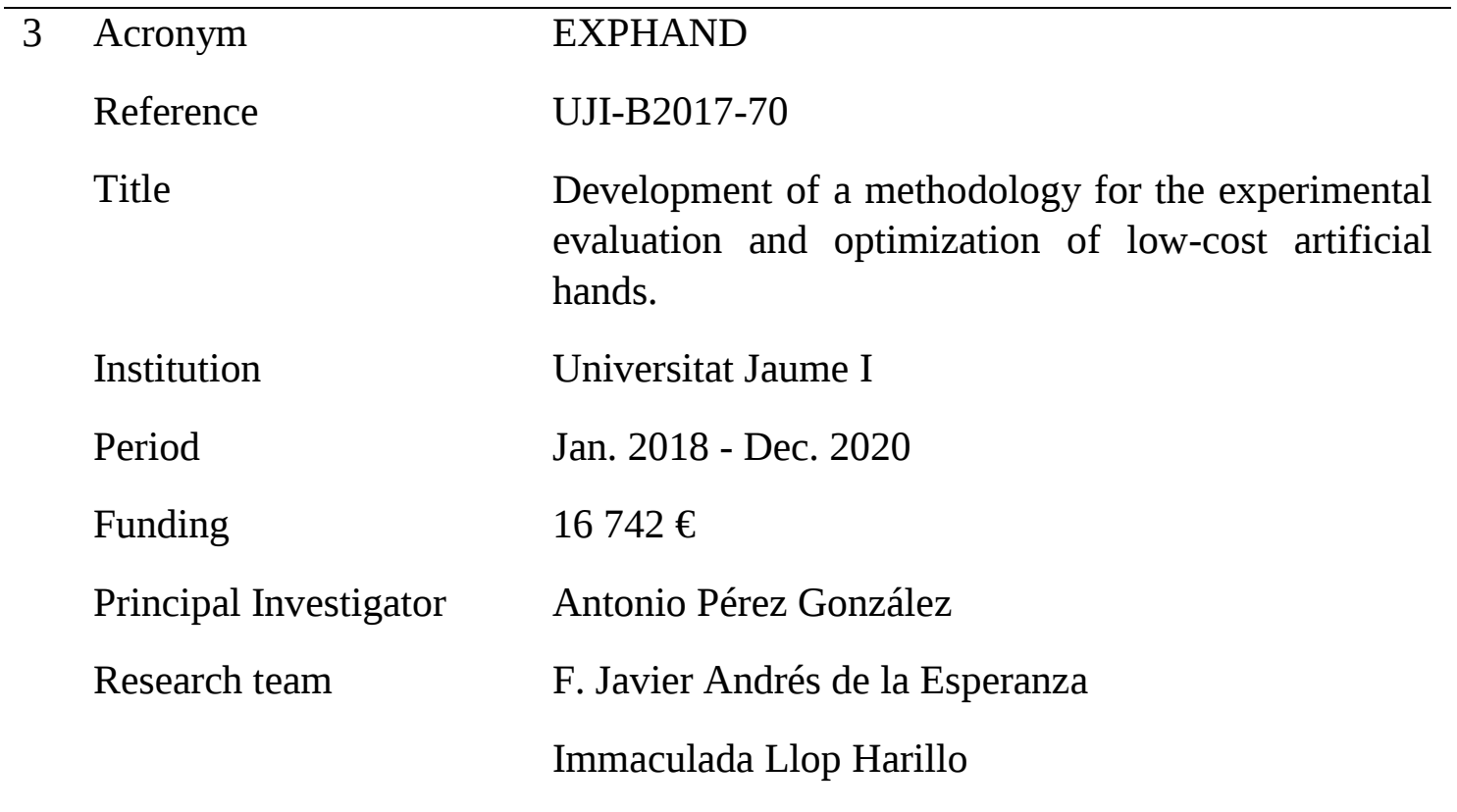

On the premise that there are no clear methodologies in the literature that allow to evaluate the manipulative capability of a specific artificial hand, the purpose of the DEVALHAND project was to develop metrics and methodologies to do so and also allowing to optimize the hand design for further development of new designs of low-cost prosthetics, actuators and controllers. The part of the objective of the BENCH-HAND project in relation with this thesis is to develop experimental benchmarks that allow comparing any artificial hand with the human hand to finally obtain an improved design of a low-cost hand prosthesis. The EXPHAND project intends to evaluate experimentally and objectively the grasping ability of prototypes of low-cost artificial hands, contributing to the improvement and optimization of existing designs. Its main objectives are: 1) to understand the effect of the mechanical design of the artificial hand with an experimental protocol that quantifies its grasping ability while being actuated and 


\section{8 | Introduction}

controlled manually by a human operator; 2) to obtain the motion synergies of the artificial hand in those conditions, which can be useful to analyze the number of actuators or motors needed for its control.

\section{Research stay}

In compliance with the requirements for applying for an international mention in my PhD, I performed a four months research stay (September to December 2017) in the High Performance Humanoid Technologies Lab $\left(\mathrm{H}^{2} \mathrm{~T}\right)$ at the Karlsruhe Institute of Technology (KIT) under the supervision of Dr. Prof. Tamim Asfour.

Most of my work during the stay was focused on improving the preliminary protocol proposed in Chapter 1, obtaining the Anthropomorphic Hand Assessment Protocol (AHAP) of Chapter 2 and published in Robotics and Autonomous Systems. During the stay I also took part in conducting the AHAP on some of the $\mathrm{H}^{2} \mathrm{~T}$ artificial hands obtaining their grasping ability score (GAS): the ARMAR-6 v1 hand, which is the first version of the robotic hand designed for the humanoid robot ARMAR-6 (Asfour et al., 2018), tested with three different configurations resulting from attachment of pads to the fingertips and palm; and the two versions of the KIT Prosthetic Hand (Weiner et al., 2018).

The Anthropomorphism Index of Mobility (AIM) presented in Chapter 4 was also obtained for the final version of the KIT Prosthetic Hand (Weiner et al., 2018) and compared with that obtained by other artificial hands.

During the stay I also participated in the development of a new experimental protocol involving cylindrical and prismatic grips with 15 objects of the KIT set (Kasper et al., 2012). The protocol was defined to evaluate robotic hands grasping the objects from a table and placing them back in the same position. Different configurations of the ARMAR-6 v1 hand, resulting from attachment of pads of different materials, were tested. This work included the determination of the friction coefficient for those materials in order to analyze the effect on the grasping capability of the artificial hand. In addition, experimental tests were performed to assess the actuation system through the comparison of the actuation force in the cables/tendons and the final force exerted by the distal phalange of the finger. These tests were performed using one of the fingers of the ARMAR-6 hand with different cables/tendons: steel rope, dyneema of different diameters and fishing line (Specitec). This work is neither part of this thesis nor has it been published. However, it was useful to better understand the possible causes of 
grasping failure observed experimentally and to analyze alternatives for improving the hand design.

During the research stay I also collaborated with some researchers of the $\mathrm{H}^{2} \mathrm{~T}$ in a review of intelligent hand prostheses, but it has not yet been published.

\section{Structure}

Since the research carried out has led to different research articles, this thesis is written as a compendium of publications. Table 2 shows the list of chapters and their correspondent publication. It should be pointed out that, according to the doctoral programme regulations, this thesis structure requires at least two published or accepted international indexed articles. On the date of writing this document, four of the chapters $(1,2,4,5)$ correspond to papers published in international journals indexed in JCR or SJR, one chapter (6) has been published as an international book chapter and two more are under review in journals indexed in JCR.

Table 2. List of chapters and their correspondent publications. * Last update: May 2020

\begin{tabular}{|c|c|c|c|}
\hline Chapter & Tittle & Publication \& Status* & Authors \\
\hline 1 & $\begin{array}{l}\text { System for the experimental } \\
\text { evaluation of } \\
\text { anthropomorphic hands. } \\
\text { Application to a new 3D- } \\
\text { printed prosthetic hand } \\
\text { prototype }\end{array}$ & $\begin{array}{l}\text { International } \\
\text { Biomechanics, 2017, } \\
4: 2,50-59\end{array}$ & $\begin{array}{l}\text { Immaculada } \\
\text { Llop-Harillo and } \\
\text { Antonio Pérez- } \\
\text { González }\end{array}$ \\
\hline 2 & $\begin{array}{l}\text { The Anthropomorphic Hand } \\
\text { Assessment Protocol } \\
\text { (AHAP) }\end{array}$ & $\begin{array}{l}\text { Robotics and } \\
\text { Autonomous Systems, } \\
\text { 2019, vol.121 }\end{array}$ & $\begin{array}{l}\text { Immaculada } \\
\text { Llop-Harillo, } \\
\text { Antonio Pérez- } \\
\text { González, Julia } \\
\text { Starke, and } \\
\text { Tamim Asfour }\end{array}$ \\
\hline 3 & $\begin{array}{l}\text { Grasping ability and motion } \\
\text { synergies in affordable } \\
\text { tendon-driven prosthetic } \\
\text { hands controlled by able- } \\
\text { bodied subjects }\end{array}$ & $\begin{array}{l}\text { Submitted to Frontiers } \\
\text { in Neurorobotics }\end{array}$ & $\begin{array}{l}\text { Immaculada } \\
\text { Llop-Harillo, } \\
\text { Antonio Pérez- } \\
\text { González, and F. } \\
\text { Javier Andrés }\end{array}$ \\
\hline
\end{tabular}


Anthropomorphism Index of Mobility for Artificial Hands

Optimization of the Kinematic Chain of the Thumb for a Hand Prosthesis Based on the Kapandji Opposition Test

\begin{abstract}
Anthropomorphism Indexes of the Kinematic Chain for Artificial Hands
\end{abstract}

\begin{abstract}
Applied Bionics and
Biomechanics, 2019, vol. 2019, 1-11
\end{abstract}

Journal of Bionic Engineering, 2020, vol. 17, 501-511

In: Computer Methods, Imaging and Visualization in Biomechanics and Biomedical Engineering, Springer. 2020, vol. 36, 271-287.

Submitted to Bioinspiration \& Biomimetics
Immaculada Llop-Harillo, Antonio PérezGonzález, and Verónica GraciaIbáñez

Immaculada Llop-Harillo, Antonio PérezGonzález, and Javier Andrés-

Esperanza

Antonio Pérez-

González and

Immaculada

Llop-Harillo
Benchmarking prosthetic hands through anthropomorphic grasping simulations
Immaculada Llop-Harillo, José

L. Iserte, and

Antonio Pérez-

González

The first part of the thesis, Chapters 1 to 3 , focuses on experimental methods and tools to: 1) assess artificial hands when performing GTs in ADL, quantifying their functionality and human-like grasping and analyzing their limitations; 2) obtain the motion synergies when a TDPH is actuated and controlled by the fingers of a healthy subject using an ABA.

In Chapter 1, a first design of a new system for the experimental evaluation of artificial hands is presented, including a preliminary design of an ABA and a protocol for obtaining grasping ability of tendon-driven hand prototypes, as well as information about the motion coordination among their fingers. A new 3D-printed TDPH prototype, called IMMA hand, was also designed and manufactured in order to perform preliminary tests to assess the validity of the system, from which some improvements were proposed for both the ABA and the protocol.

In Chapter 2, a formal experimental benchmark (AHAP) is presented, improving the preliminary protocol proposed in Chapter 1. Its major 
improvements were a new scoring system, the definition of the criteria for grasp correctness, and its statistical validation. It was successfully applied to humanoid and prosthetic hands and its reliability, consistency and responsiveness were statistically analyzed verifying its robustness across raters and proving also the comparability of the results across different hands and testing conditions.

In Chapter 3, different affordable 3D-printed TDPHs are confronted with the AHAP proposed in Chapter 2, using an improved version of the ABA used in Chapter 1 . Several healthy subjects using their own fingers actuated the artificial hands by means of the ABA. The excursion of the tendons of the different hands was registered and correlation and principal component analyses were used to obtain the motion synergies. The use of the ABA allowed taking advantage of the human brain control, thus focusing the comparison in the mechanical design of the hands. The subject effect on the hand control was also analyzed. The principal components obtained provided useful information for the design of the transmission or control systems that may be used in order to underactuate these hands.

In the second part of the thesis, Chapters 4 to 6 , I present some analytical methods complementary to the experimental ones presented in previous chapters. They include the definition of anthropomorphism indexes, the use of simplified models of the hand and optimization techniques. All these indexes are based on the comparison with the human hand kinematic structure or grasping behavior. They are mainly oriented to be applied in the initial stages of the design process in order to improve the anthropomorphism and functionality of artificial hands, but they are also valid as a method to assess and grade human-likeness of already existing artificial hands.

In Chapter 4, I propose an index, named AIM, with a straightforward computation, to evaluate the anthropomorphism of artificial hands based on the comparison of the topology of the whole hand, joints and DoFs, and the possibility to control those DoFs independently. The index weights the relevance of each DoF according to the results obtained in a human grasp experiment on the most important GTs in ADL.

In Chapters 5, different methods are proposed to compare the kinematic chain (KC) of the artificial hand with that of the human hand. The main parameters defining the kinematics of the hand, such as the orientation of the joint axes, the range of motion of the joints or the dimension of the phalanges, are considered. Based on a simplified model of the KC, three complementary indexes were defined for the comparison of: 1) the 


\section{2 | Introduction}

parameters of the KC; 2) the reachable workspace; and 3) common grasping postures.

As the thumb plays a key role in the performance of the hand for grasping and manipulating objects, in Chapter 6 I present a computational method to optimize the whole thumb KC (base placement, link lengths and joint orientation angles) based on the hand performance in the Kapandji opposition test (Kapandji, 1986). As a case study, the method was applied to the improvement of the thumb KC of the IMMA hand presented in Chapter 1.

Finally, I propose in Chapter 7 a new method for adapting a grasping simulation framework, OpenRAVE (Diankov, 2010), to the evaluation of anthropomorphic artificial hand designs. A preliminary approach to a new simulation benchmark is presented. It involves the use of human knowledge for the generation of efficient grasp hypotheses and the definition of a new metric to assess stability and human likeness adapting to the simulation environment the experimental benchmark presented in Chapter 2. This method was applied to compare thirty design alternatives of the IMMA hand presented in Chapter 1. Moreover, the new simulation benchmark was compared with a previous approach (León et al., 2014, 2012; Rubert et al., 2017) showing clear improvements in computation time and realism of grasping postures. The results of the original hand at both benchmarks, experimental (AHAP) and simulated, were also compared.

\section{Other publications}

Apart from the publications that compose this thesis (Table 2), I contributed in other publications (Table 3) related to the research presented here.

Table 3. Other publications.

\begin{tabular}{|c|c|}
\hline Repository & $\begin{array}{l}\text { Antonio Pérez-González and Immaculada Llop-Harillo (2019) 3D } \\
\text { models of the objects selected (from YCB set) for the different grasp } \\
\text { types in the Anthropomorphic Hand Assessment Protocol (AHAP), DOI: } \\
\text { 10.5281/zenodo.3560735 }\end{array}$ \\
\hline Congress & $\begin{array}{l}\text { Immaculada Llop Harillo, Antonio Pérez González, Jesús Cantero Ramis } \\
\text { y Francisco Javier Andrés (2019) Evaluación y comparación de manos } \\
\text { protésicas de impresión 3D mediante el Anthropomorphic Hand } \\
\text { Assessment Protocol (AHAP), IX Reunión del Capítulo Español de la } \\
\text { Sociedad Europea de Biomecánica (ESB), ISBN: 978-84-09-15896-6 }\end{array}$ \\
\hline
\end{tabular}


Congress Antonio Pérez-González and Immaculada Llop-Harillo (2019) Optimization of the kinematic chain of the thumb for a hand prosthesis based on the Kapandji opposition test, 16th International Symposium on Computer Methods in Biomechanics and Biomedical Engineering and 4th Conference on Imaging and Visualization (CMBBE 2019)

Congress Immaculada Llop-Harillo, Antonio Pérez-González and Jesús CanteroRamis (2019) Analysis of motion synergies in grasping tasks for a 6-DoF tendon-driven prosthetic hand, 16th International Symposium on Computer Methods in Biomechanics and Biomedical Engineering and 4th Conference on Imaging and Visualization (CMBBE 2019)

Congress Immaculada Llop-Harillo, Antonio Pérez-González, Jesús CanteroRamis, F. Javier Andrés and José Fuentes (2019) Effect of the subject on the control of tendon-driven prosthetic hands with an able-bodied adaptor, 25th Congress of the European Society of Biomechanics (ESB 2019), ISBN: 978-3-903024-96-0

Congress Immaculada Llop Harillo y Antonio Pérez González (2018) Evaluación y comparación del antropomorfismo de manos artificiales, VIII Reunión del Capítulo Español de la Sociedad Europea de Biomecánica (ESB), ISBN: 978-84-09-06674-2

Congress César Corella Mora, Antonio Pérez González e Immaculada Llop Harillo

(Awarded) (2018) Índice de movilidad de una mano protésica basado en el índice de Kapandji modificado, VIII Reunión del Capítulo Español de la Sociedad Europea de Biomecánica (ESB), ISBN: 978-84-09-06674-2

Congress Immaculada Llop Harillo, Antonio Pérez González y F.J. Andrés de la

(Awarded) Esperanza (2018) Comparación de la cadena cinemática de manos antropomorfas artificiales con la de la mano humana, XXII Congreso Nacional de Ingeniería Mecánica (CNIM 2018), ISSN: 0212-5072

Congress Immaculada Llop-Harillo, Verónica Gracia-Ibáñez and Antonio PérezGonzález (2018) Analysis of anthropometric dimensions and joints range of motion of the human hand for application to the design of hand prostheses, 8th World Congress of Biomechanics (WCB 2018)

Congress Immaculada Llop-Harillo, Carlos Rubert and Antonio Pérez-González (2018) Computation of grasp quality metrics in OpenHand Simulator to improve a 3D printed prosthetic hand, 15th International Symposium on Computer Methods in Biomechanics and Biomedical Engineering and 3rd Conference on Imaging and Visualization (CMBBE 2018), ISBN: 978-989-99424-5-5

Congress Immaculada Llop-Harillo, Antonio Pérez-González and Verónica Gracia-Ibáñez (2017) Anthropomorphism index of mobility for hand prostheses, XXVI Congress of the International Society of Biomechanics

Congress Verónica Gracia-Ibañez, Margarita Vergara, Joaquín L. Sancho-Bru, Denis Mottet, Isabelle Laffont, Karima Bahkti, Immaculada Llop-Harillo 


\begin{tabular}{ll}
\hline \multirow{4}{*}{ Congress } & $\begin{array}{l}\text { and Antonio Pérez-González (2017) Mimicking kinematic sinergies } \\
\text { underlying activities of daily living for rehabilitation, XXVI Congress of } \\
\text { the International Society of Biomechanics }\end{array}$ \\
& $\begin{array}{l}\text { Immaculada Llop-Harillo, Antonio Pérez-González, Verónica Gracia- } \\
\text { Ibáñez and Carlos Rubert (2017) Design and evaluation of } \\
\text { anthropomorphic hands, Summer School on Soft Manipulation 2017 }\end{array}$ \\
Congress & $\begin{array}{l}\text { Immaculada Llop-Harillo, Antonio Pérez-González and Carlos Rubert } \\
\text { (2017) Anthropomorphism index of opposition for hand prostheses, 23rd }\end{array}$ \\
Congress of the European Society of Biomechanics \\
Congress & $\begin{array}{l}\text { Immaculada Llop-Harillo and Antonio Pérez-González (2017) IMMA } \\
\text { Hand, https://sites.google.com/a/uji.es/devalhand/imma-hand }\end{array}$ \\
& $\begin{array}{l}\text { Immaculada Llop-Harillo and Antonio Pérez-González (2016) 3D } \\
\text { hand prostheses, Engineering the Upper Limb (Institution of Mechanical }\end{array}$ \\
& Engineers) \\
Immaculada Llop y Antonio Pérez (2016) Sistema para la evaluación \\
experimental de diseños de prótesis de mano de bajo coste, VI Reunión \\
del Capítulo Español de la Sociedad Europea de Biomecánica (ESB), \\
ISBN: 978-84-697-6124-3
\end{tabular}

\section{References}

Asfour, T., Kaul, L., Wachter, M., Ottenhaus, S., Weiner, P., Rader, S., Grimm, R., Zhou, Y., Grotz, M., Paus, F., Shingarey, D., Haubert, H., 2018. ARMAR6: A Collaborative Humanoid Robot for Industrial Environments, in: 2018 IEEE-RAS 18th International Conference on Humanoid Robots (Humanoids), pp. 447-454.

Belter, J.T., Segil, J.L., Dollar, A.M., Weir, R.F., 2013. Mechanical design and performance specifications of anthropomorphic prosthetic hands: A review. The Journal of Rehabilitation Research and Development, 50(5), 599-617.

Biagiotti, L., Lotti, F., Melchiorri, C., Vassura, G., 2003. How Far Is the Human Hand? A Review on Anthropomorphic Robotic End-effectors, University of Bologna, Italy.

Bonsignorio, F., Del Pobil, A.P.D.P., Messina, E., 2014. Fostering Progress in Performance Evaluation and Benchmarking of Robotic and Automation Systems. IEEE Robotics \& Automation Magazine, 21(1), 22-25.

Calli, B., Walsman, A., Singh, A., Srinivasa, S., Abbeel, P., Dollar, A.M., 2015. Benchmarking in Manipulation Research: Using the Yale-CMU-Berkeley Object and Model Set. IEEE Robotics \& Automation Magazine, 22(3), 36-52.

Cepriá-Bernal, J., Pérez-González, A., Mora, M.C., Sancho-Bru, J.L., 2016. Grip 
force and force sharing in two different manipulation tasks with bottles. Ergonomics, 1-10.

Controzzi, M., Cipriani, C., Carrozza, M.C., 2014. Design of Artificial Hands: A Review, in: Springer Tracts in Advanced Robotics. pp. 219-246.

Diankov, R., 2010. Automated Construction of Robotic Manipulation Programs. Architecture, Ph.D., 1-263.

Duong, T., Wagner, B., Abraham, T., Davidson, M., Bains, G., Daher, N., Friedrich, A., 2017. Comparative Study of Functional Grasp and Efficiency between a 3D-Printed and Commercial Myoelectric Transradial Prosthesis Using Able-Bodied Subjects: A Pilot Study. Journal of Prosthetics and Orthotics, 29(3), 112-118.

Falco, J., Van Wyk, K., Liu, S., Carpin, S., 2015. Grasping the Performance: Facilitating Replicable Performance Measures via Benchmarking and Standardized Methodologies. IEEE Robotics \& Automation Magazine, 22(4), 125-136.

Feix, T., Romero, J., Ek, C.H., Schmiedmayer, H.-B., Kragic, D., 2013. A Metric for Comparing the Anthropomorphic Motion Capability of Artificial Hands. IEEE Transactions on Robotics, 29(1), 82-93.

Gracia-Ibáñez, V., Sancho-Bru, J.L., Vergara, M., 2018. Relevance of grasp types to assess functionality for personal autonomy. Journal of Hand Therapy, 1(31), 102-110.

Gracia-Ibáñez, V., Vergara, M., Buffi, J.H., Murray, W.M., Sancho-Bru, J.L., 2017a. Across-subject calibration of an instrumented glove to measure hand movement for clinical purposes. Computer Methods in Biomechanics and Biomedical Engineering, 20(6), 587-597.

Gracia-Ibáñez, V., Vergara, M., Sancho-Bru, J.L., Mora, M.C., Piqueras, C., 2017b. Functional range of motion of the hand joints in activities of the International Classification of Functioning, Disability and Health. Journal of Hand Therapy, 30(3), 337-347.

Gracia Ibañez, V., 2016. Contribution to hand functional assessment based on its kinematics. Ph.D., Universitat Jaume I, Castelló de la Plana.

Jiang, L., Liu, Y., Yang, D., Liu, H., 2018. A synthetic framework for evaluating and designing an anthropomorphic prosthetic hand. Journal of Bionic Engineering, 15(1), 69-82.

Kapandji, A., 1986. Clinical opposition and reposition test of the thumb. Annales de Chirurgie de la Main, 5(1), 67-73.

Kasper, A., Xue, Z., Dillmann, R., 2012. The KIT object models database: An object model database for object recognition, localization and manipulation in service robotics. The International Journal of Robotics Research, 31(8), 927934. 
León, B., Morales, A., Sancho-Bru, J., 2014. From Robot to Human Grasping Simulation, Cognitive Systems Monographs, Cognitive Systems Monographs. Springer International Publishing, Cham.

León, B., Rubert, C., Sancho-Bru, J., Morales, A., 2013. Evaluation of prosthetic hands prehension using grasp quality measures, in: 2013 IEEE/RSJ International Conference on Intelligent Robots and Systems, pp. 3501-3506.

León, B., Sancho-Bru, J.L., Jarque-Bou, N.J., Morales, A., Roa, M.A., 2012. Evaluation of human prehension using grasp quality measures. International Journal of Advanced Robotic Systems, 9.

Li, S., Ma, X., Liang, H., Gorner, M., Ruppel, P., Fang, B., Sun, F., Zhang, J., 2019. Vision-based Teleoperation of Shadow Dexterous Hand using End-toEnd Deep Neural Network, in: 2019 IEEE International Conference on Robotics and Automation (ICRA), pp. 416-422.

Liarokapis, M. V., Artemiadis, P.K., Kyriakopoulos, K.J., 2013. Quantifying anthropomorphism of robot hands, in: 2013 IEEE International Conference on Robotics and Automation, pp. 2041-2046.

Liarokapis, M. V., Artemiadis, P.K., Kyriakopoulos, K.J., 2012. Functional Anthropomorphism for human to robot motion mapping, in: Proceedings IEEE International Workshop on Robot and Human Interactive Communication, Paris, France, pp. 31-36.

Liu, Y., Yang, D., Jiang, L., Liu, H., 2015. A synthetic framework for evaluating the anthropomorphic characteristics of prosthetic hands, in: IEEE/ASME International Conference on Advanced Intelligent Mechatronics, AIM.

Mio, R., Sanchez, M., Valverde, Q., 2018. Mechanical Testing Methods for BodyPowered Upper-Limb Prostheses: A Review, in: 2018 IEEE 18th International Conference on Bioinformatics and Bioengineering (BIBE), pp. 170-176.

OpenAI, Andrychowicz, M., Baker, B., Chociej, M., Józefowicz, R., McGrew, B., Pachocki, J., Petron, A., Plappert, M., Powell, G., Ray, A., Schneider, J., Sidor, S., Tobin, J., Welinder, P., Weng, L., Zaremba, W., 2018. Learning Dexterous In-Hand Manipulation. CoRR.

Pérez-González, A., Vergara, M., Sancho-Bru, J.L., 2013. Stiffness map of the grasping contact areas of the human hand. Journal of Biomechanics, 46(15), 2644-2650.

Puig, J.E.P., Rodriguez, N.E.N., Ceccarelli, M., 2008. A Methodology for the Design of Robotic Hands with Multiple Fingers. International Journal of Advanced Robotic Systems, 5(2), 22.

Quispe, A.H., Amor, H. Ben, Christensen, H.I., 2018. A Taxonomy of Benchmark Tasks for Robot Manipulation, in: Springer Proceedings in Advanced Robotics. pp. 405-421.

Roa, M.A., Suárez, R., 2014. Grasp quality measures: review and performance. 
Autonomous Robots, 38(1), 65-88.

Rubert, C., León, B., Morales, A., Sancho-Bru, J., 2017. Characterisation of Grasp Quality Metrics. Journal of Intelligent \& Robotic Systems, 89(3-4), 319-342.

Rubert, C., Morales, A., 2016. Comparison between grasp quality metrics and the anthropomorphism index for the evaluation of artificial hands, in: 2016 6th IEEE International Conference on Biomedical Robotics and Biomechatronics (BioRob), pp. 1352-1357.

Sancho-Bru, J.L., 2000. Model biomecànic de la mà orientat al disseny d'eines manuals. Ph.D.

Sancho-Bru, J.L., Jarque-Bou, N.J., Vergara, M., Pérez-González, A., 2014. Validity of a simple videogrammetric method to measure the movement of all hand segments for clinical purposes. Proceedings of the Institution of Mechanical Engineers. Part H, Journal of engineering in medicine, 228(2), 182-189.

Sancho-Bru, J.L., Mora, M.C., León, B.E., Pérez-González, A., Iserte, J.L., Morales, A., 2012. Grasp modelling with a biomechanical model of the hand. Computer methods in biomechanics and biomedical engineering, 17(4), 297310.

Sancho-Bru, J.L., Pérez-González, A., Vergara, M., Giurintano, D.J., VergaraMonedero, M., 2003. A 3D biomechanical model of the hand for power grip. Journal of biomechanical engineering, 125(1), 78-83.

Simon, A.M., Turner, K.L., Miller, L.A., Hargrove, L.J., Kuiken, T.A., 2019. Pattern recognition and direct control home use of a multi-articulating hand prosthesis, in: IEEE International Conference on Rehabilitation Robotics. pp. 386-391.

ten Kate, J., Smit, G., Breedveld, P., 2017. 3D-printed upper limb prostheses: a review. Disability and Rehabilitation: Assistive Technology, 12(3), 300-314.

Vergara, M., Agost, M.J., Gracia-Ibáñez, V., 2018. Dorsal and palmar aspect dimensions of hand anthropometry for designing hand tools and protections. Human Factors and Ergonomics in Manufacturing \& Service Industries, 28(1), 17-28.

Vergara, M., Sancho-Bru, J.L., Gracia-Ibáñez, V., Pérez-González, A., 2014. An introductory study of common grasps used by adults during performance of activities of daily living. Journal of Hand Therapy, 27, 225-234.

Weiner, P., Starke, J., Hundhausen, F., Beil, J., Asfour, T., 2018. The KIT Prosthetic Hand: Design and Control, in: 2018 IEEE/RSJ International Conference on Intelligent Robots and Systems (IROS), pp. 3328-3334. 



\section{Chapter 1}

System for the experimental evaluation of anthropomorphic hands. Application to a new 3D-printed prosthetic hand prototype 

This chapter corresponds exactly to the published paper: System for the experimental evaluation of anthropomorphic hands. Application to a new 3D-printed prosthetic hand prototype Immaculada Llop-Harillo and Antonio Pérez-González International Biomechanics, 2017, 4(2), 50-59

\begin{abstract}
In the present study, we propose a new actuation device and protocol for testing the grasping performance of low-cost 3D-printed hand prototypes. The actuation device is connected to the forearm of a healthy user and allows him to use his thumb and fingers to control any prototype moved by up to six tendons attached to this device. The protocol includes grasping actions on 24 different objects using eight typical grasp types to obtain a grasping ability score and information about the coordination of motion among the fingers. This study also presents a new design for a low-cost 3D-printed prosthetic hand, called the IMMA hand. Preliminary tests were performed with the IMMA hand and the actuation device on two subjects, using the protocol, to assess the validity of the device for the experimental evaluation of hand prototypes in early design stages. The analysis of the results of these tests shows that the coordination of motions among fingers is quite similar for both subjects, indicating a similar control of the artificial hand. Index and ring finger motions are highly correlated in over half of the grasp actions performed for both subjects.
\end{abstract}

\title{
Keywords
}

Anthropomorphic hand, grasping evaluation, hand prostheses, design, 3D printing, finger coordination

\section{Introduction}

The evaluation of grasping ability is essential to improve current anthropomorphic hand designs in fields like prosthetics and robotics. In the robotics community, many grasp quality metrics have been proposed in the literature to evaluate the grasp quality of manipulators. A recent survey by Roa and Suárez (Roa and Suárez, 2015) identified up to 24 different grasp quality metrics. These metrics play a key role in the analytical approach to 


\section{2 | Chapter 1}

the grasp-planning problem in robotics, often referred to as grasp synthesis, and also help quantify desirable properties like dexterity, force-closure, stability and equilibrium (Sahbani et al., 2012; Shimoga, 1996). Comparison of the grasp metrics between artificial hands and the human hand has recently been proposed as a method to evaluate prosthetic hands (León et al., 2013).

However, the metrics cited above are analytical and focused on evaluating the stability of the object grasped in a particular grasping posture. They are mainly based on geometrical information such as the contact points and the contact normal between the hand and the object. These metrics do not evaluate the performance of the hand globally and they are obtained from simulations. Moreover, few analyses have been carried out to assess the ability of these hands to emulate the grasp modes of the human hand. In fact, evaluation of mechanical hands globally and not only for specific grasping postures has been dealt with only poorly in the literature. A recent empirical study by Belter et al. (Belter et al., 2013) compared the characteristics of different prosthetic hands, but without defining outcome measures. The recent study by Feix et al. (Feix et al., 2013) is, to our knowledge, the only such work that attempts to define an index which aims to compare the motion capability of robotic and prosthetic hands with that of the human hand, although it is somewhat limited, its computation being based only on the comparison of the position and orientation of the distal phalanges in different possible hand poses. Moreover, it is an analytical study, and important aspects for grasping such as friction, surface roughness and contact compliance are difficult to consider with analytical approaches.

Attention has also been paid to experimental approaches that aim to overcome the limitations observed with analytical approaches (Balasubramanian et al., 2012; Kim et al., 2012), considering effects such as friction, deformation, accuracy and sensitivity to positioning errors. In prosthetics and rehabilitation, several experimental protocols have been defined to evaluate the hand or the prosthetic hand function. Examples of these tests include the Sollerman Hand Function Test (Sollerman and Ejeskär, 1995), the Southampton Hand Assessment Procedure (SHAP) (Light et al., 2002) and the Action Arm Research Test (McDonnell, 2008). However, these tests are designed as clinical evaluation tools for application to subjects wearing the prosthesis or in the process of rehabilitation. The development of specific systems and protocols for the experimental evaluation of hand prototypes in the design phase is envisaged as a complementary tool to analytical metrics for improving anthropomorphic hand design. 
Currently, however, the design of anthropomorphic hands is not based on sound grasp metrics or standard experimental protocols. Orthopaedic companies base their designs mainly on previous experience and user feedback, with an emphasis on grip modes and aesthetics. Developments in robotic hands like the DLR hand (Grebenstein et al., 2012) are moving towards a greater number of degrees of freedom (DOF), but their weight and size are necessarily greater than that of the human hand. Furthermore, price is not among the main factors considered in any of the previous approaches. More recent open designs of anthropomorphic hands, such as the Open Hand Project (Gibbard, 2013), Flexy-Hand (Gyrobot, 2014) and others, were compiled and analysed by the authors on a website (Biomechanics and Ergonomics, 2016). These designs are moving towards low-cost models that enable self-manufacture using 3D printing technologies and customization.

Experimental tests and protocols for evaluating or comparing different mechanical hand designs globally in terms of their anthropomorphism or grasping ability and which are focused on improving the design of the artificial hands are scarce in the scientific literature. The development of simulation tools, benchmarks and protocols for evaluating hand designs numerically and experimentally is crucial for their future design. The prehensile ability of an artificial hand depends basically on three groups of factors: its mechanical design, the capacity of its actuators or motors and its control system. In this study we assumed that it is desirable to decouple these three groups of factors to achieve an effective improvement of the design of artificial hands. Thus, in a first stage it is desirable to have a good understanding of the effect of the mechanical design of the hand and therefore it is also advisable that the actuation and the control of the hand prototypes are performed manually by a human operator. Accordingly, in the present study we propose a new actuation device and protocol for testing the grasping ability of different hand prototypes experimentally. The actuation device has been designed to be placed on the forearm of a healthy subject, thus allowing him to use his thumb and fingers to control any hand prototype attached to this device moved by up to six tendons. The tendon-driven action is similar to that used in the human hand and intuitive for a human subject operating the hand. It also transmits some force-feedback information to the subject, which is very interesting to help achieve successful grasping. The use of a human controller eliminates the distorting effect that different control systems could have on the results when comparing different hands, thus allowing the hands to be compared only from the point of view of their mechanical design. If a human is in the loop actuating the device, we are 


\section{4 | Chapter 1}

close to having the best possible control (the human mind) and the best actuator for grasping (the human hand), although with the limitations imposed by the actuation device. Moreover, the actuation device designed in this study also permits the registration of the displacement of each driving cable during the tests, thereby allowing the coordination movements among the fingers to be studied. The information thus obtained can be used to consider the best option for the actuation of the artificial hand by motors, in a similar way to how it is performed by a human operator. The protocol to evaluate the hand prototypes includes grasping actions on 24 different objects using eight typical grasp types. In the present study, we also present a new design of a low-cost 3D-printed prosthetic hand with six DOF actuated by tendons, which was used to test the actuation device. Both the prosthetic hand and the actuation device are manufactured with 3D printing because this technology is typically used in low-cost hand prostheses and allows easy interchange of designs, which improves the comparability of the results. Preliminary tests were performed with this hand and the actuation device, using the proposed protocol, in order to assess the validity of the device for the experimental evaluation of hand prototypes in early design stages.

\section{Materials and methods}

\subsection{Actuation device}

A new device was designed that allowed a healthy subject to perform manual actuation of different low-cost 3D-printed models of artificial hands in order to test their grasping ability. Actuation by a healthy subject has the advantage that the control strategy for grasping is performed by the user's brain, thus allowing different artificial hand prototypes to be compared from the point of view of the mechanical configuration and design, without the interference of different control strategies. The actuation device was designed taking into account the following specifications: 1) easy, fast, safe and secure attachment of the device to the forearm of a healthy adult subject; 2) adaptable to different hand-arm sizes of the users; 3) simple attachment of different artificial hands to the device must be possible and strong enough to resist a moment of $3 \mathrm{Nm}$; 4) intuitive and comfortable actuation of the artificial hand attached to the device (with up to six DOF) using tendons connected to the subject's fingers and thumb; 5) the device must include sensors to measure and record the excursion of each actuating tendon during any grasping action; and 6) low weight and preferably manufactured by $3 \mathrm{D}$ printing technology. 
Considering these specifications, several conceptual solutions were proposed for the device and preliminary prototypes were built to evaluate these conceptual solutions, mainly in reference to the method of actuation for the tendons. One of the options was to use a glove with external tendons whose excursion depended on the motion of the hand of the subject wearing this glove. The external tendons of the glove must be connected to the corresponding tendons of the artificial hand. This concept would permit an intuitive actuation of the artificial hand, but after building a first prototype this option was discarded because of the problems encountered when it came to obtaining a reliable solution for the attachment of the tendons to the glove and because of the difficulty involved in adapting a single glove size to the different hand sizes of the subjects who have to actuate the device, which affected the excursion of the tendons and hence the motion of the artificial hand. Finally, a concept based on the use of rings for the actuation of the tendons of the artificial hand was considered more reliable. Attachment of the device to the forearm of the healthy user was solved with a 3D-printed part and Velcro ${ }^{\circledR}$ straps, which allowed it to be attached in less than two minutes and to remain in place without feelings of pain or discomfort throughout the whole test. The actuation device was manufactured using 3D printed technology. As mentioned above, this technology is common for low-cost 3D-printed hand prostheses and suitable for a low-weight solution. Moreover, it allows easy replication of the device by other research groups or developers, thereby promoting more accurate comparisons between hand designs. The connection of different artificial hands to the device is straightforward, requiring only the redesign of a customized connecting part at the end of the device. Our initial design is based on a press fit solution, which allows simple and sufficiently strong attachment of the low-cost prosthetic hand presented in this study. The solution adopted to measure the excursion of the tendons was the use of linear potentiometers connected to these tendons. These sensors allow the actuation of each finger to be compared during the tests and the analysis of synergies between the motion of the fingers, which can be used to reduce the number of motors for a prosthesis and simplify its control. An Arduino Uno board was used to collect the signal of the potentiometers and send it to a computer through a USB communication cable. This board was installed in a sensors box with the six linear potentiometers. The total weight of the actuation device including the sensors and the electronic board was $472 \mathrm{~g}$. Figure 1 shows the prototype of the device that was designed, with a hand prototype attached to it. Figure 2 shows the actuation device, in a different view, worn by a healthy subject during a grasping task. 


\section{6 | Chapter 1}

The actuation device allows the testing of hand prototypes actuated with up to six tendons by connecting these tendons to the corresponding rings of the device.

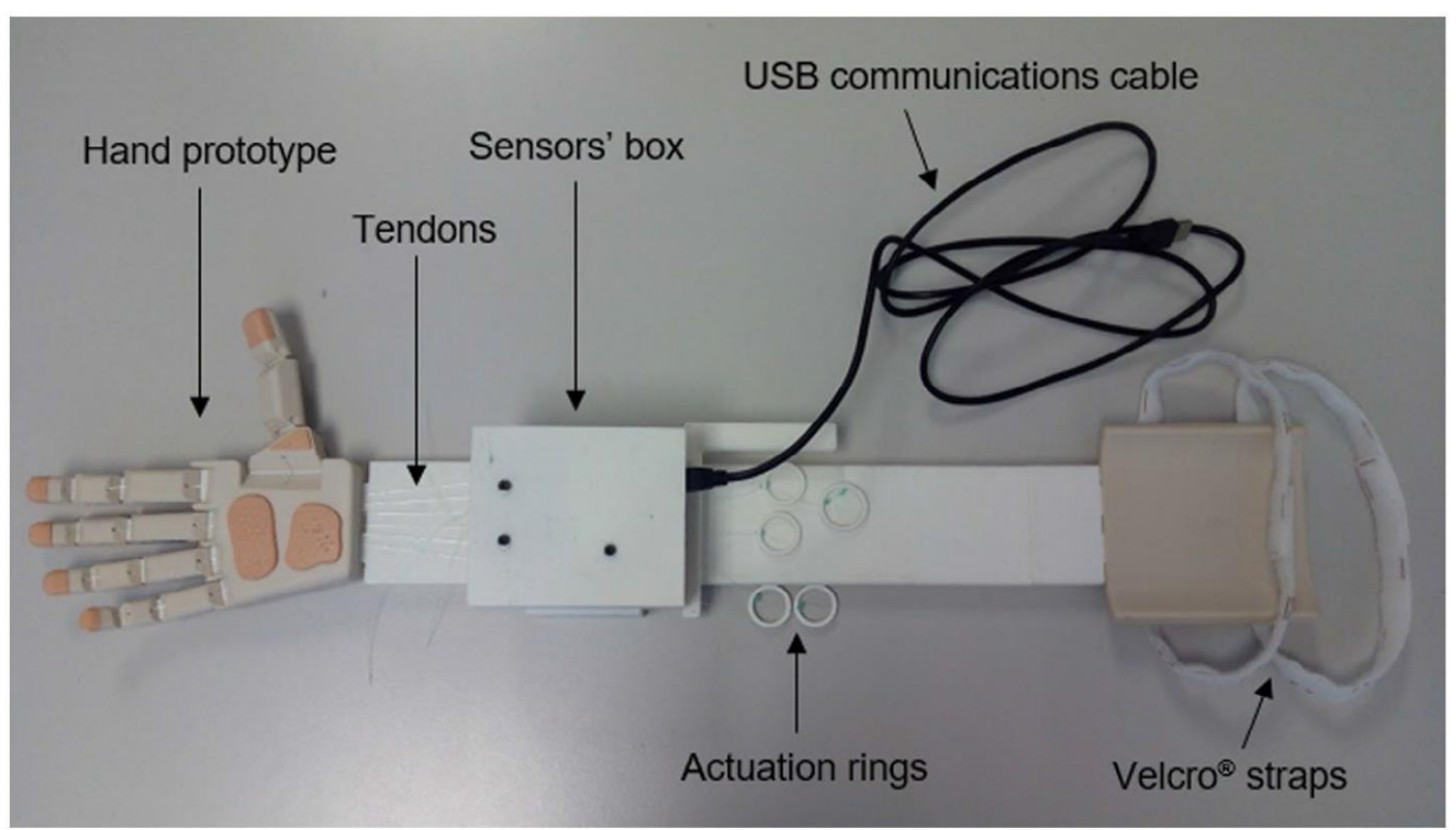

Figure 1. Actuation device with the IMMA hand prototype attached to it. The different components are shown: hand prototype, tendons, sensors box, actuation rings, USB communications cable and Velcro ${ }^{\circledR}$ straps for attachment to the forearm.

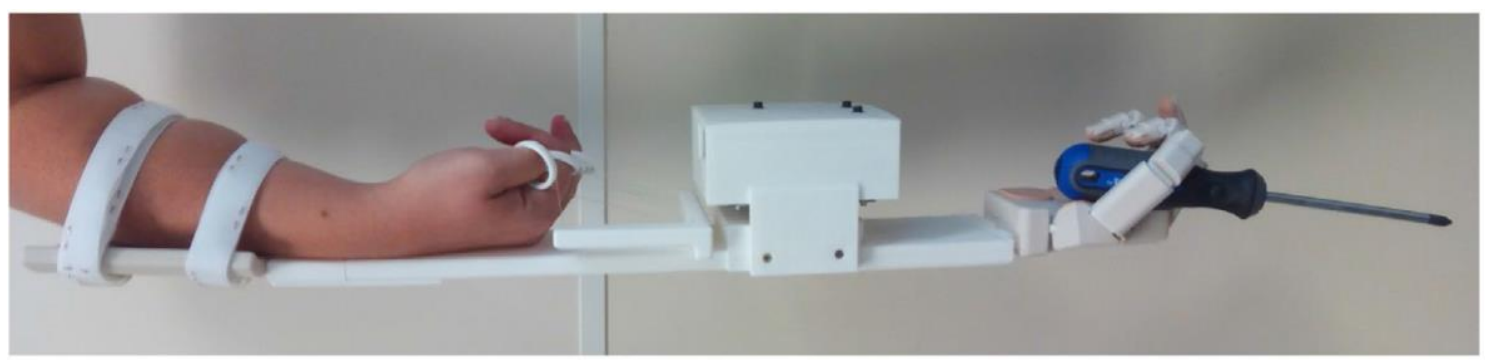

Figure 2. Device actuated by a subject during a grasping task.

\subsection{Prosthetic hand}

A new low-cost anthropomorphic prosthetic hand has been designed. The following specifications were considered for the design: 1) tendon-driven hand with six DOFs: independent flexion of the thumb and each finger and abduction motion of the thumb (the combination of flexion-extension and abduction-adduction movements of the thumb allows opposition of the thumb to orient the thumb distal phalanx to the distal phalanges of the fingers); 2) use of elastic elements to drive extension movements when the tendons are released; 3) natural rest position when not actuated; 4) simple 
and low-cost manufacturing and assembly, based on 3D printing technology; 5 ) main dimensions between the $50^{\text {th }}$ percentiles of the male and female human hands; 6) friction coefficient and stiffness in the main contact areas of the palm and fingers similar to those of the human hand; 7) total weight no greater than that of the human hand; and 8) aesthetical appearance.

Some ideas were taken from previous developments to design a new prosthetic hand under these specifications. The design of the finger joints was inspired by the Flexy-Hand model (Gyrobot, 2014), using elastic components, which connect the two phalanges of the corresponding joint (for interphalangeal joints) or the proximal phalange to the palm (for the metacarpophalangeal joints of the fingers and the carpometacarpal joint of the thumb). This solution simplifies the assembly of the hand and at the same time allows easy return to the rest position when the tendons are slack, without the need for additional springs. The orientation of the axes for the carpometacarpal joint of the thumb was taken as similar to that used in the ADA hand (Open Bionics, 2016), but in our design the flexion and abduction movements of the thumb are actuated separately, as in the Tact hand (Slade et al., 2015) or the Dextrus hand (Gibbard, 2013). The orientation of the fingers was taken as similar to that of the ADA hand (Open Bionics, 2016) and the K1 hand (Keuster, 2015).

Different materials based on different combinations of polylactic acid (PLA) and thermoplastic polyurethane (TPU) were used in the construction of the hand to obtain the proper stiffness and friction coefficient in each area in order to improve the grasping ability: PLA SOFT-Flexible (mixture of PLA and TPU using hexamethylene diisocyanate) was used for the palm and phalanges; NinjaFlex ${ }^{\circledR}$ (special formulation of TPU with high flexibility and durability) was used for the elastic joints; FilaFlex ${ }^{\circledR}$ (based on TPU with additives) was used for inserts located in the finger pads and some areas of the palm, which are the parts of the hand with more contact with the objects, because of its good compliance and greater friction coefficient. Different tests were performed using a dynamometer to characterize the mechanical behaviour of the different parts of the hand and validate the specifications. To obtain the approximate contact stiffness we used a cylindrical indentor with a flat end (3.8 $\mathrm{mm}$ in diameter) following a procedure similar to that described in a previous work (Pérez-González et al., 2013). The mean stiffness obtained for the different parts of the hand was, respectively, $5.1 \mathrm{~N} / \mathrm{mm}$ for FilaFlex ${ }^{\circledR}$ inserts used in the distal phalanges, $13.9 \mathrm{~N} / \mathrm{mm}$ for the main body of the hand and phalanges made of PLA SOFT-Flexible and $6.3 \mathrm{~N} / \mathrm{mm}$ for the FilaFlex ${ }^{\circledR}$ inserts used in the palm. The rotational stiffness 


\section{8 | Chapter 1}

of the joints was obtained by measuring the force necessary to rotate the joint until close to its limit and dividing the torque applied by the angle rotated. The stiffness obtained for the finger joints was dependent on the joint width, with an average value of $1.8 \mathrm{Nmm} / \mathrm{rad}$ per mm of width of the joint. The friction coefficient of the FilaFlex ${ }^{\circledR}$ inserts and that of the PLA SOFTFlexible surfaces of the hand against aluminium were obtained by measuring the force needed to slide a prismatic block of this material in contact with the corresponding part of the hand. An approximate friction coefficient of 0.63 was obtained for the FilaFlex ${ }^{\circledR}$ inserts, and 0.22 in the case of the PLA SOFTFlexible.

Four nylon fishing lines, with a diameter of $0.5 \mathrm{~mm}$ and a strength of $135 \mathrm{~N}$, were used as tendons for flexing the fingers and two more for flexing and abducting the thumb, with an appropriate routing through the phalanges. A knot was used to attach the tendon to the distal phalanges, this knot being hidden below the inserts used in the distal phalanges to improve the aesthetics. The dimensions of the fingers and palm of the hand were selected so to be between the $50^{\text {th }}$ percentiles of human male and female hands, based on data obtained in the authors' research group, with a hand length (from the most proximal palmar point to the tip of the middle finger) of $184.4 \mathrm{~mm}$ and a hand width (at the metacarpal heads) of $80 \mathrm{~mm}$. The total weight of the hand was $131.5 \mathrm{~g}$ and the cost of its 3D-printing material was less than $10 €$. The prototype of the hand, referred to as the IMMA hand, is shown in Figure 3.
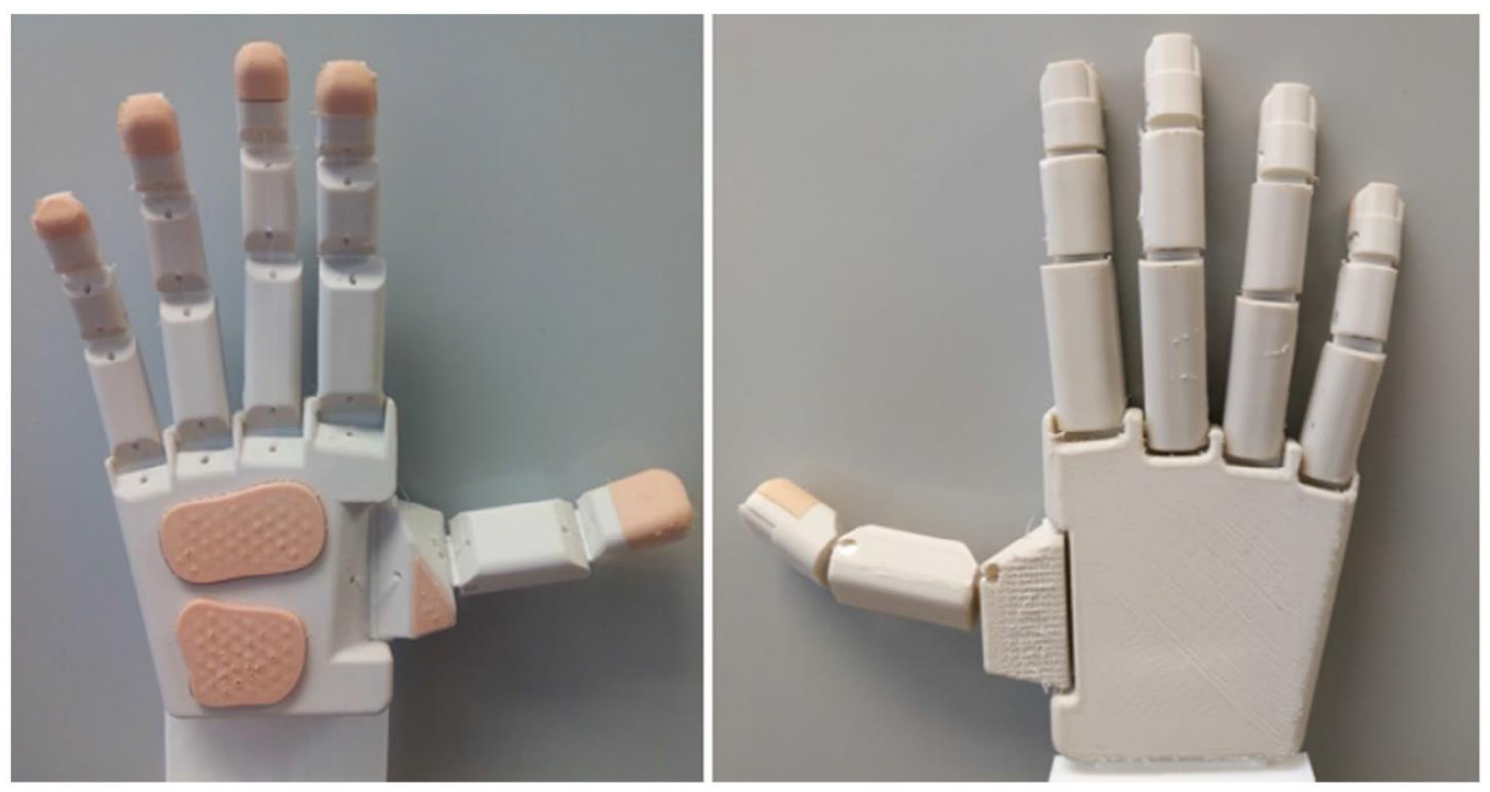

Figure 3. Prototype of the IMMA hand. 


\subsection{Protocol for testing hand prototypes}

A standard protocol for testing hand prototypes would help obtain experimental evidence with which to compare the grasping performance that can be reached with different hand designs. The use of the actuation device developed in the present study, connected to different hand prototypes, allows straightforward comparison of different hands. As a healthy operator is used to actuate the hand prototypes with this device by pulling the rings, the control issues are avoided and only the mechanical aspects of the design (materials, geometry, friction coefficients, mechanical advantage, efficiency, etc.) are considered in the evaluation and comparison of the artificial hands.

In order to establish the protocol, a group of objects and grasping tasks must be selected. We decided to choose the objects from a standard set of objects recently proposed for establishing benchmarks in manipulation research, the Yale-CMU-Berkeley Object and Model Set (Calli et al., 2015), because this set has been widely distributed among the robotics and biomechanics research community. The set is composed of 73 different objects including some elements typically found in activities of daily living (ADL). From this set we selected a subset of 24 objects, divided into eight groups of three objects, with each group corresponding to objects typically grasped with one of eight different grasping postures or grasp types (GT). We partially based the selection of these eight GT on the results of a previous field study conducted in the authors' research group about grasps used in ADL (Vergara et al., 2014) and on previous research by other authors in the area of rehabilitation and prosthetics (Light et al., 2002; Sollerman and Ejeskär, 1995). Figure 4 shows the eight different GT considered (pulp pinch, lateral pinch, diagonal volar grip, cylindrical grip, extension grip, tripod pinch, spherical grip and hook grip) and the three objects from the YCB set used for each of them. The three objects for each GT were selected with a view to varying the size, shape and weight. For the hook grasp a combination of two objects in the set, rope and coloured wood blocks, were selected as one of the objects, the rope being used as a handle for lifting the wood blocks container.

For the test, the healthy subject, wearing the actuation device with the hand prototype attached to it, was instructed to grasp different objects for close to three seconds. The subject was in a standing position during the test and the test operator held the objects close to the artificial hand, in the correct orientation for performing the desired GT. The subject was instructed to try to use, as much as possible, the GT corresponding to each object. During the 


\section{0 | Chapter 1}

test the operator registered the success or failure to keep the grasp without the object falling and scored the results, assigning 1 point if the grasp was completed successfully in the first trial, 0.6 points if completed successfully in the second trial, 0.4 if completed in the third trial, 0.2 points if completed with a grasping posture other than the one specified and 0 points if the grasp was unsuccessful. Scores for the three objects with each grasping posture were added to obtain the final score for this grasping posture. Scores for all the objects/tasks were then added to obtain the final score for the artificial hand. Normalized scores can be obtained by dividing by the maximum possible scores. The electronics of the actuation device also registered the excursion of the tendons during the test duration as well as the time spent on the task. Figure 5 shows some examples of task executions with different objects included in the protocol.

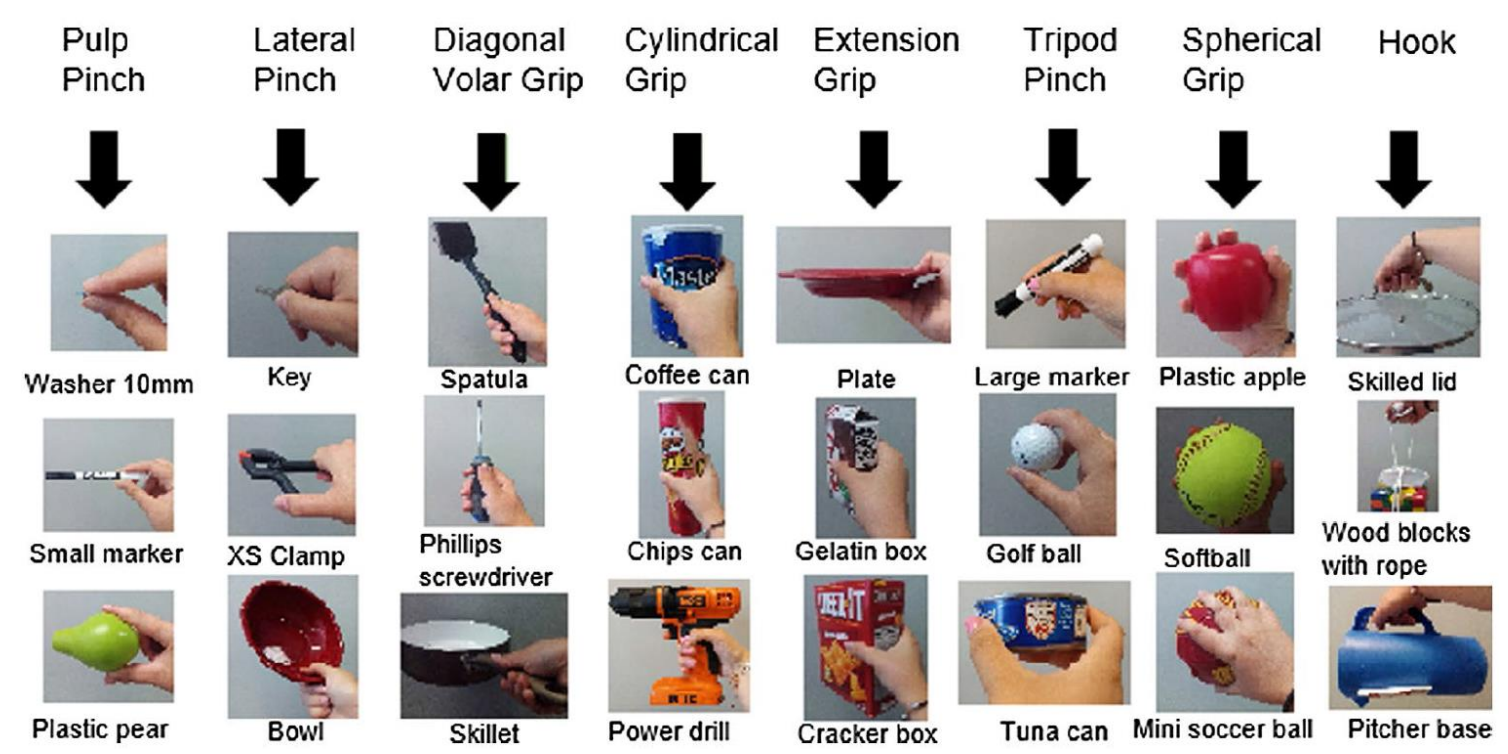

Figure 4. Grasping types and objects from the YCB set used in the protocol.

\subsection{Preliminary tests}

A preliminary experiment was undertaken with the aim of testing the proposed protocol, the performance of the actuation device and the performance of the IMMA hand. Two adult male subjects, members of the research group (age 50 and 43, hand length $18.5 \mathrm{~cm}$ and $20.0 \mathrm{~cm}$, hand width $9.5 \mathrm{~cm}$ and $9.0 \mathrm{~cm}$, respectively), without any previous hand pathology, participated in the experiment, which was approved by the Ethics Committee of the university. They followed the protocol as described in the previous section, performing the grasping actions with the 24 objects in random order, in the same session. The excursion of the actuation tendons registered by the electronic box of the device during the tests was analysed with the aim of 
finding correlations or synergies among the different actuating tendons. For this analysis only the motion of the tendons from the extended position to the final grasping posture was considered, but not the return to the extended position, which should be performed by the elastic joints because this is the relevant portion of the motion to analyse the possible effect of the synergies on the reduction of the number of actuators for the artificial hand. For the purposes of the analysis, a correlation coefficient between two tendons higher than a threshold of 0.9 was taken as high enough (within the limit of the possible experimental errors) to consider the possibility of using the same actuator for two different tendons.

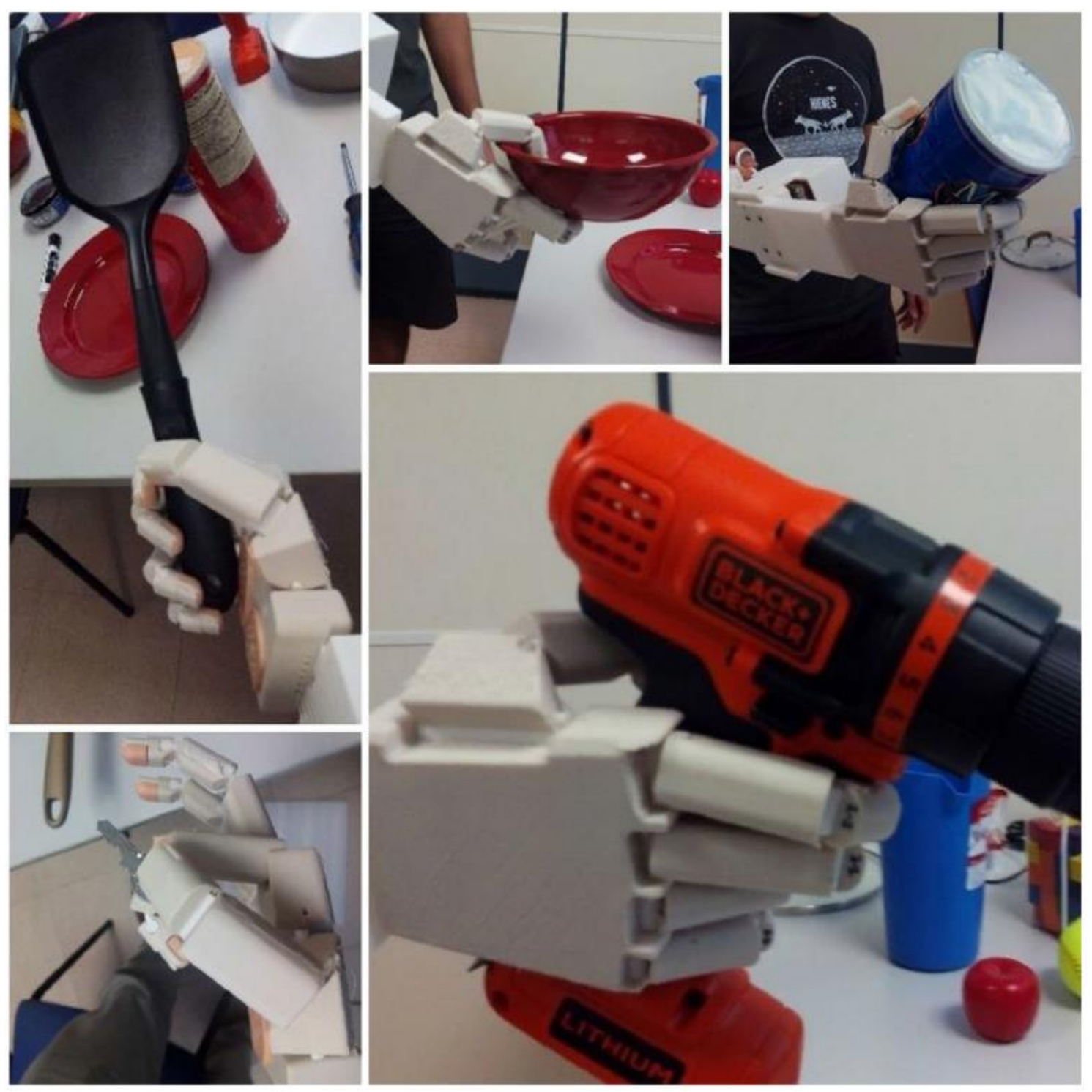

Figure 5. Examples of grasps performed by a subject wearing the actuation device with the IMMA hand. 


\section{2 | Chapter 1}

\section{Results}

The preliminary tests confirmed the validity of the actuation device for actuating the IMMA hand prototype. The device was easily attached to the forearm of both subjects in less than two minutes and the hand prototype was attached to the device simply with the press fit, which could resist the moment generated when lifting the heaviest object in the set (around $3 \mathrm{Nm}$ ). The connection of the tendons to the electronics box took around 30 minutes, and was the slowest action in this attachment process. Both subjects found it easy to control the artificial hand using the rings connected to their own fingers, although they found it somewhat difficult to control the thumb abduction. Even though the device was light, some fatigue was reported by the subjects at the end of the test.

Table 1 shows the objects for which each of the two subjects failed in the grasp, grouped by GT. Pulp pinch was the most difficult GT, followed by spherical grip. All the objects corresponding to lateral pinch, diagonal volar grip, cylindrical grip, tripod pinch and hook grip were grasped successfully by both subjects. Following the scoring method proposed in the protocol, the grasping ability obtained by the IMMA hand was $71 \%$ with the first subject and $75 \%$ with the second subject.

Table 1. Objects from the YCB set grouped by grasp type, for which any of the subjects failed in the grasping task.

\begin{tabular}{ccc}
\hline Grasp type (GT) & Subject 1 & Subject 2 \\
\hline Pulp pinch & Plastic pear*, small & Plastic pear*, small \\
& marker, washer & marker, washer \\
Extension grip & Plate & - \\
Spherical grip & Softball, & Softball, \\
& mini soccer ball & mini soccer ball \\
\hline
\end{tabular}

${ }^{*}$ The object was grasped but the GT used was considered a lateral pinch

Figure 6 shows an example of the evolution of the excursions for the six tendons used in the anthropomorphic hand. An excursion of $0 \mathrm{~mm}$ corresponds to the rest position with the hand extended by the flexible joints. The plateau in the central part of the curves corresponds to the stable grasp posture maintained around three seconds. The final part of the curves after releasing the object indicates that the hand prosthesis did not recover the initial extended posture autonomously because the extending moment introduced by the flexible joints of the IMMA hand is unable to overcome the friction force existing in the linear potentiometers of the actuation device. 


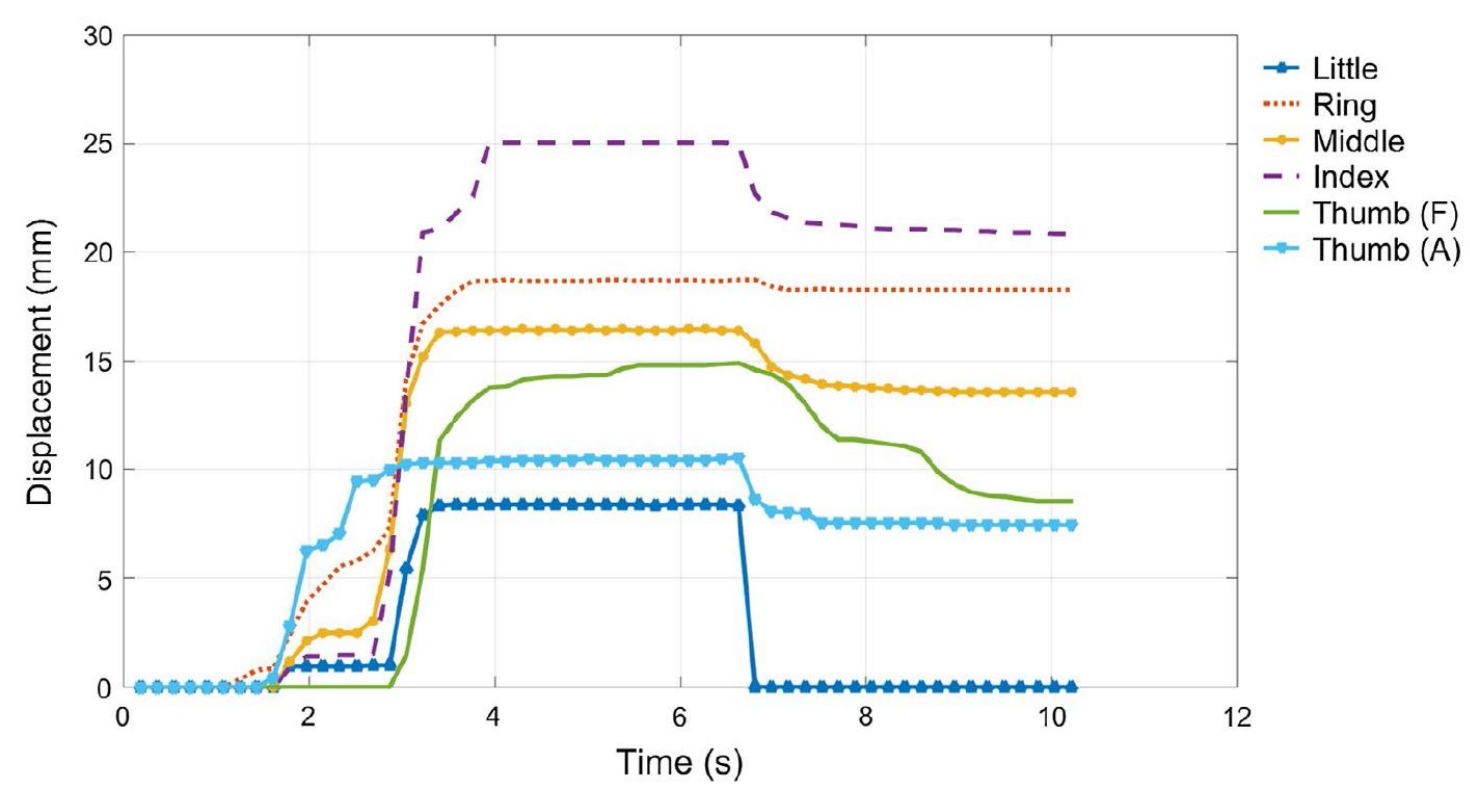

Figure 6. Example of excursion of the tendons during the grasp of the power drill with the IMMA hand operated by Subject 2 using the actuation device.

Table 2 shows the frequency of cases, among the successful grasps for each subject, for which the correlation coefficient between the excursions of each pair of tendons was higher than 0.9. The most frequently correlated motions corresponded to those of the index and ring fingers, for both subjects, followed by those of the middle and ring fingers. The correlation between both tendons (flexion and abduction) moving the thumb is also significant. The ring finger is the one presenting the highest motion correlation with other fingers. Thumb flexion is highly correlated for near one third of the cases with index finger flexion and near one quarter of the cases with ring finger flexion, the correlation with middle finger flexion not being frequent.

Table 2. Frequency of cases, among successful grasps for each subject, for which the correlation coefficient between the excursions of each pair of tendons was higher than 0.9 for a) Subject 1 and b) Subject 2. Darker colours correspond to higher correlations.

a) Subject 1

\begin{tabular}{ccccccc}
\hline & Little & Ring & Middle & Index & $\begin{array}{c}\text { Thumb } \\
\text { flexion }\end{array}$ & $\begin{array}{c}\text { Thumb } \\
\text { abduction }\end{array}$ \\
\hline Little & $100 \%$ & $0 \%$ & $0 \%$ & $0 \%$ & $0 \%$ & $0 \%$ \\
Ring & & $100 \%$ & $39 \%$ & $56 \%$ & $22 \%$ & $0 \%$ \\
Middle & & & $100 \%$ & $17 \%$ & $0 \%$ & $0 \%$ \\
Index & & & & $100 \%$ & $33 \%$ & $6 \%$ \\
Thumb flexion & & & & & $100 \%$ & $28 \%$ \\
Thumb abduction & & & & & $100 \%$ \\
\hline
\end{tabular}




\section{4 | Chapter 1}

b) Subject 2

\begin{tabular}{ccccccc}
\hline & Little & Ring & Middle & Index & $\begin{array}{c}\text { Thumb } \\
\text { flexion }\end{array}$ & $\begin{array}{c}\text { Thumb } \\
\text { abduction }\end{array}$ \\
\hline Little & $100 \%$ & $0 \%$ & $0 \%$ & $0 \%$ & $0 \%$ & $0 \%$ \\
Ring & & $100 \%$ & $60 \%$ & $65 \%$ & $25 \%$ & $35 \%$ \\
Middle & & & $100 \%$ & $35 \%$ & $10 \%$ & $10 \%$ \\
$\begin{array}{c}\text { Index } \\
\text { Thumb flexion } \\
\text { Thumb abduction }\end{array}$ & & & $100 \%$ & $35 \%$ & $15 \%$ \\
\hline
\end{tabular}

\section{Discussion}

In this study we have presented a new actuation device, which can be used for the experimental evaluation of artificial hands. This evaluation can be employed to assess the goodness of other analytical metrics to give an index of the grasping capabilities of an artificial hand design, but can also provide complementary information in early design stages, which is helpful for improving the design. The device can be actuated by a healthy subject in order to move the fingers and thumb of the artificial hand, through cables connected to up to six tendons of the artificial hand. As the control of the hand through the device is carried out by the human operator, the possible differences in control methods for the artificial hand are not included in the evaluation. Moreover, manual actuation is helpful to provide some feedback about the force exerted on the object. To our knowledge, this is the first development of a device to manually actuate artificial hand prototypes for assessing their grasping capabilities. Other studies used previously instrumented gloves or other devices for teleoperating robotic hands (Farry et al., 1996; Xu and Todorov, 2016), but in these methods the artificial hand is moved with motors and the devices used for teleoperation were used only to send signals to actuate the motors of the hand. Our solution of manual operation has the advantage of offering good feedback to the user in order to improve control while grasping. Additionally, the system provides information about the actuation coordination performed in the different DOFs of the artificial hand to grasp each object, which can be very useful for designing under-actuated hands, because those DOFs actuated in a very coordinated manner for most of the objects are candidates for being actuated with the same motor.

From the preliminary tests, some limitations have been observed in the current design of the actuation device. The friction introduced by the potentiometers of the device should be reduced because it prevents the extension of the fingers of the IMMA hand when the tendons are released. 
However, this fact does not breach any of the specifications proposed for the hand because it is able to return easily to the rest position when the tendons are slack if the hand is not connected to the actuation device. Moreover, this limitation does not invalidate the results of the experiments because only the motion from the initial extended position to the grasp posture is considered for the analysis of the correlations between the actuating tendons. The following improvements are envisaged for future versions of the device:

- A redesign of the device for a configuration in parallel to the arm of the user instead of as a prolongation of it, thereby allowing a final position of the artificial hand located at the same distance from the body as the user's hand but displaced towards the sagittal plane. This improvement would allow a more natural and ergonomic actuation of the artificial hand, especially for grasping objects resting on a table, and would also reduce the moment transferred by the device to the forearm when grasping heavy objects.

- A more comfortable and ergonomic method for connecting the cables to the user's fingers, especially for the thumb, and adaptable to different sizes of the user's hand.

- A change in the method of measurement of the excursions of the tendons to reduce the friction introduced by the linear potentiometers on the tendons.

- The use of wireless connection between the device and the computer to improve the portability of the device.

A protocol has been proposed for the experimental testing of artificial hands for use in robotics or prosthetics. The protocol is based on the manual actuation of the artificial hand with the actuation device in order to undertake grasping actions with different objects and grasp types. Three different objects from the YCB set (Calli et al., 2015) were selected for each of eight different GT, all of them characteristic of the main grasping postures used with the human hand. With this protocol we are able to obtain a score for the grasping ability of each prosthetic hand as well as information about the coordination of motion among the fingers that can be used for further analysis of the motors required to actuate the prosthesis and its control strategy. The protocol is centred on grasping actions, although it could be improved in the future to include other non-grasping postures, such as point and platform, which a multigrasp prosthetic hand should also be capable of achieving according to previous studies (Balasubramanian and Santos, 2014). 


\section{6 | Chapter 1}

A new design for a low-cost 3D-printed prosthetic hand, called the IMMA hand (Llop-Harillo and Pérez-González, 2017), has been presented in this study. The hand has six degrees of freedom, two of them for the thumb to allow opposition, actuated by tendons, and combines different materials to obtain an appropriate friction coefficient and compliance in the contact areas. The stiffness obtained in the finger and palm inserts is comparable to that measured in the distal phalanges of the fingers (Pérez-González et al., 2013). Moreover, a similar friction coefficient to that observed in the human hand (O’Meara and Smith, 2001) was obtained between the IMMA hand inserts and aluminium.

The preliminary tests performed with the actuation device indicate a fairly good grasping capability, except for pulp pinch and spherical grip. A change in the orientation of the thumb joints could improve these limitations and is going to be analysed in the next version of this hand design.

The analysis of the results of the preliminary tests performed on two subjects shows that the coordination of motions among fingers is quite similar for both subjects (Table 2), indicating a similar control of the artificial hand by both subjects. Index and ring finger motions are highly correlated in more than half of the grasp actions performed, thumb flexion being more correlated with these two fingers than with the middle finger. A recent study about coordination of motion among the joints of the human hand in dexterity tests and ADL has observed the highest correlations between index and middle metacarpophalangeal joints (Gonzalez-Sanchez et al., 2016). Our results also show significant coordination between the flexion of the index and middle fingers, but a higher coordination has been observed between the ring and index fingers, although the experiments are not totally comparable. Thumb flexion and abduction are correlated for near $30 \%-40 \%$ of the grasping tasks, but this correlation can be due to the fact that both motions are actuated with the same ring attached to the subject's thumb. This particularity of the actuation device is considered a limitation that makes the control of the thumb of the artificial hand difficult and should be improved for further developments of this device.

\section{Conclusion}

We have proposed a framework for the experimental evaluation of robotic and prosthetic hands in order to assess their grasping capabilities when controlled by a human operator. The actuation device developed can be used easily by healthy users to test the artificial hands, providing 
information about the control strategy employed during grasping because it registers the excursion of the actuating tendons during the grasp action. The control strategy followed by different users has been shown to be similar in a preliminary test with two users. Future work will focus on improving the design of the actuation device to make it more ergonomic and on testing and comparing different artificial hands using this framework to obtain conclusions for improving future designs of low-cost prosthetic hands.

\section{Funding}

This work was supported by the Spanish Ministry of Economy and Competitiveness and ESF [grant number BES-2015-076005]; Spanish Ministry of Economy and Competitiveness and ERDF [grant number DPI2014-60635-R].

\section{References}

Balasubramanian, R., Santos, V.J., 2014. The Human Hand as an Inspiration for Robot Hand Development. Springer Tracts in Advanced Robotics.

Balasubramanian, R., Xu, L., Brook, P.D., Smith, J.R., Matsuoka, Y., 2012. Physical Human Interactive Guidance: Identifying Grasping Principles From Human-Planned Grasps. IEEE Transactions on Robotics, 28(4), 899-910.

Belter, J.T., Segil, J.L., Dollar, A.M., Weir, R.F., 2013. Mechanical design and performance specifications of anthropomorphic prosthetic hands: A review. The Journal of Rehabilitation Research and Development, 50(5), 599-617.

Biomechanics and Ergonomics, 2016. DevalHand-Hands Info. https://sites.google.com/site/devalhandhandsinfo/

Calli, B., Walsman, A., Singh, A., Srinivasa, S., Abbeel, P., Dollar, A.M., 2015. Benchmarking in Manipulation Research: The YCB Object and Model Set and Benchmarking Protocols, http://arxiv.org/abs/1502.03143.

Farry, K.A., Walker, I.D., Baraniuk, R.G., 1996. Myoelectric teleoperation of a complex robotic hand. IEEE Transactions on Robotics and Automation, 12(5), 775-788.

Feix, T., Romero, J., Ek, C.H., Schmiedmayer, H.B., Kragic, D., 2013. A metric for comparing the anthropomorphic motion capability of artificial hands. IEEE Transactions on Robotics, 29(1), 82-93.

Gibbard, J., 2013. Open Hand Project. www.openhandproject.org

Gonzalez-Sanchez, V., Rowson, J., Yoxall, A., 2016. Analysis of finger movement coordination during the Variable Dexterity Test and comparative activities of daily living. International Journal of Therapy and Rehabilitation, 23(10), 


\section{8 | Chapter 1}

481-491.

Grebenstein, M., Chalon, M., Friedl, W., Haddadin, S., Wimbock, T., Hirzinger, G., Siegwart, R., 2012. The hand of the DLR Hand Arm System: Designed for interaction. The International Journal of Robotics Research, 31(13), 1531-1555.

Gyrobot, 2014. Flexy-Hand. http://www.thingiverse.com/thing:380665

Keuster, E., 2015. The K1 Hand. https://3dprint.nih.gov/discover/3dpx-001705

Kim, J., Iwamoto, K., Kuffner, J.J., Ota, Y., Pollard, N.S., 2012. Physically-based grasp quality evaluation under uncertainty, in: Proceedings - IEEE International Conference on Robotics and Automation, pp. 3258-3263.

León, B., Rubert, C., Sancho-Bru, J., Morales, A., 2013. Evaluation of prosthetic hands prehension using grasp quality measures, in: IEEE International Conference on Intelligent Robots and Systems. pp. 3501-3506.

Light, C.M., Chappell, P.H., Kyberd, P.J., 2002. Establishing a standardized clinical assessment tool of pathologic and prosthetic hand function: Normative data, reliability, and validity. Archives of Physical Medicine and Rehabilitation, 83(6), 776-783.

Llop-Harillo, I., Pérez-González, A., 2017. IMMA hand (Devalhand project). https://sites.google.com/a/uji.es/devalhand/imma-hand.

McDonnell, M., 2008. Action research arm test. The Australian journal of physiotherapy, 54(3), 220.

O’Meara, D.M., Smith, R.M., 2001. Static friction properties between human palmar skin and five grabrail materials. Ergonomics, 44(11), 973-988.

Open $\quad$ Bionics, 2016. Ada hand V1.1. https://www.openbionics.com/obtutorials/ada-v1-assembly

Pérez-González, A., Vergara, M., Sancho-Bru, J.L., 2013. Stiffness map of the grasping contact areas of the human hand. Journal of Biomechanics, 46(15), 2644-2650.

Roa, M.A., Suárez, R., 2015. Grasp quality measures: review and performance. Autonomous Robots, 38(1), 65-88.

Sahbani, A., El-Khoury, S., Bidaud, P., 2012. An overview of 3D object grasp synthesis algorithms. Robotics and Autonomous Systems, 60(3), 326-336.

Shimoga, K.B., 1996. Robot Grasp Synthesis Algorithms: A Survey. The International Journal of Robotics Research, 15(3), 230-266.

Slade, P., Akhtar, A., Nguyen, M., Bretl, T., 2015. Tact: Design and performance of an open-source, affordable, myoelectric prosthetic hand, in: Proceedings IEEE International Conference on Robotics and Automation. pp. 6451-6456.

Sollerman, C., Ejeskär, A., 1995. Sollerman Hand Function Test: A Standardised Method and its Use in Tetraplegic Patients. Scandinavian Journal of Plastic and Reconstructive Surgery and Hand Surgery, 29(2), 167-176. 
Vergara, M., Sancho-Bru, J.L., Gracia-Ibáñez, V., Pérez-González, A., 2014. An introductory study of common grasps used by adults during performance of activities of daily living. Journal of Hand Therapy, 27, 225-234.

Xu, Z., Todorov, E., 2016. Design of a highly biomimetic anthropomorphic robotic hand towards artificial limb regeneration, in: Proceedings - IEEE International Conference on Robotics and Automation. pp. 3485-3492. 



\section{Chapter 2}

The Anthropomorphic Hand Assessment Protocol (AHAP) 

This chapter corresponds exactly to the published paper:

The Anthropomorphic Hand Assessment Protocol (AHAP) Immaculada Llop-Harillo, Antonio Pérez-González, Julia Starke, and Tamim Asfour

Robotics and Autonomous Systems, 2019, 121, 103259

\begin{abstract}
The progress in the development of anthropomorphic hands for robotic and prosthetic applications has not been followed by a parallel development of objective methods to evaluate their performance. The need for benchmarking in grasping research has been recognized by the robotics community as an important topic. In this study we present the Anthropomorphic Hand Assessment Protocol (AHAP) to address this need by providing a measure for quantifying the grasping ability of artificial hands and comparing hand designs. To this end, the AHAP uses 25 objects from the publicly available Yale-CMU-Berkeley Object and Model Set thereby enabling replicability. It is composed of 26 postures/tasks involving grasping with the eight most relevant human grasp types and two non-grasping postures. The AHAP allows to quantify the anthropomorphism and functionality of artificial hands through a numerical Grasping Ability Score (GAS). The AHAP was tested with different hands, the first version of the hand of the humanoid robot ARMAR-6 with three different configurations resulting from attachment of pads to fingertips and palm as well as the two versions of the KIT Prosthetic Hand. The benchmark was used to demonstrate the improvements of these hands in aspects like the grasping surface, the grasp force and the finger kinematics. The reliability, consistency and responsiveness of the benchmark have been statistically analyzed, indicating that the AHAP is a powerful tool for evaluating and comparing different artificial hand designs.
\end{abstract}

\title{
Keywords
}

Assessment, benchmark, grasping, prosthetic hand, robotic hand, test protocol 


\section{4 | Chapter 2}

\section{Highlights}

- Benchmarking protocol to quantify the grasping ability of anthropomorphic hands.

- The Grasping Ability Score quantifies functionality and human-like grasping.

- The reliability, consistency and responsiveness have been statistically analyzed.

- The protocol has been applied successfully to humanoid and prosthetic hands.

\section{Introduction and related work}

Within the last years there has been considerable progress in the development of anthropomorphic artificial hands both for robotic applications (Controzzi et al., 2014; Puig et al., 2008) and prosthetic hands (Belter et al., 2013). 3D-printing technologies have facilitated the advancement of low-cost artificial hands (ten Kate et al., 2017). However, there has not been a parallel development of objective methods to evaluate or compare the performance of the different hand designs. The necessity of specific benchmarking in this field has been recognized by several standardization organizations as the National Institute of Standards and Technology (NIST) (Falco et al., 2015) and different researchers (Bonsignorio et al., 2014; Calli et al., 2015; Mio et al., 2018; Quispe et al., 2018). It has also been a recurrent topic for specific workshops in the last editions of international robotics conferences. In general, standardized performance testing or benchmarking is a fundamental tool that is crucial for the progress of any activity of research and development. It provides the ability to replicate and compare quantified results to enhance understanding of the effectiveness of an approach for improving product designs.

The development of anthropomorphic hands in the robotics community seeks to achieve highly dexterous end-effectors and a human like appearance, especially in service robots and human-robot cooperation (Kemp et al., 2007; Liarokapis et al., 2012; Puig et al., 2008). The complexity of these anthropomorphic hands challenges the design of the grasping performance benchmarks. Additionally, any benchmark applied to a physical hand evaluates the combination of the mechanical design and the applied control strategy. The wide range of developed hardware, following a variety of design objectives, as well as the different underlying control algorithms 
make a fair comparison hard. Moreover, the differences between robotic and prosthetic hands should be considered if we want to establish a common benchmark for hand design. In prosthetic hands the need for standalone hardware poses challenging restrictions on the actuation and embedded mechatronics. On the other hand, control parts, which are vital for the success of robotic grasping as for example the correct pre-grasp pose of the arm, are not part of the prosthetic control system as they are performed by the user of the prosthesis.

Early benchmarks in robotic manipulation were proposed for teleoperation tasks including Duplo blocks as standardized objects to grasp and manipulate (Yokokohji et al., 2003). A general metric for the grasping skill of planar grippers was later presented using cylindrical test objects of varying size (Kragten et al., 2010). As an initiative of the NIST, Falco et al. (Falco et al., 2015) proposed a framework for standardized benchmarking of robotic hands. They classified the performance tests into three levels: component tests, system tests and functional tests. Several benchmarks proposed for hand exoskeletons are also inspired by these robotic gripper evaluation methods (Bostelman et al., 2017). A metric comparing complex control algorithms on arbitrary hardware was presented by Van Wyk et al. applying a peg-in-hole-task evaluation (Van Wyk et al., 2018). Recently, Quispe et al. (Quispe et al., 2018) proposed a general taxonomy for benchmarking of manipulation tasks for service robotics and describe recommendations about how to define useful testing protocols.

In contrast, performance assessment of prosthetic hands has been based on specifically designed protocols or questionnaires. Lindner et al. (Lindner et al., 2010) compared the contents of outcome measures that have been developed to evaluate the functional performance among upper limb prosthesis users. The comparison was based on the International Classification of Functioning, Disability and Health (ICF) with an emphasis on the psychometric properties. Although many of the selected measures were based on interviews, the authors highlighted that other hand function measures "such as the Southampton Hand Assessment Procedure (SHAP) (Light et al., 2002), Box and Block test (Mathiowetz et al., 1985), Jebsen Taylor hand function test (Stern, 1992) and Assisting Hand Assessment (AHA) (Krumlinde-Sundholm et al., 2007), which are designed primarily for measuring hand function, are potentially useful measures for upper limb prosthetics” (Lindner et al., 2010). In the literature there are few studies that compare prostheses using the Box and Block test (Duong et al., 2017) and the SHAP (Belter et al., 2016). 


\section{6 | Chapter 2}

Some works have addressed the question of defining indices for measuring the anthropomorphism of artificial hands in robotics or prosthetics (Feix et al., 2013; Liarokapis et al., 2013; Liu et al., 2015). The comparison of the workspace of the fingertips or joints is used in (Feix et al., 2013; Liarokapis et al., 2013) in order to measure the ability to mimic the human hand. The study of Liu et al. (Liu et al., 2015) concentrates especially on the mechanical properties, splitting them into physical and actuation properties of the prosthetic hands. While these metrics give a good overview over the design specifications of prosthetic hands, they pay only little attention to the grasping capabilities and do not include grasping tests.

A key point for developing widely accepted benchmarks for grasping is to use a commonly available set of objects. Several sets of virtual objects have been proposed for grasp planning research in service robotics (Kasper et al., 2012; Singh et al., 2014), but the availability of the physical objects is sometimes limited (Calli et al., 2015). With the Yale-CMU-Berkeley Object and Model Set (YCB set) (Calli et al., 2015) - a collection of physically available objects of daily living for robotic grasping and manipulation benchmarks - the comparability of grasping experiments on robot hands was notably facilitated. In addition, the authors also proposed a structure for protocols and benchmarks and implemented several test procedures including the YCB Gripper Assessment Benchmark to assess the capabilities of robotic grippers using objects from the shape and tool categories of the YCB set. This protocol was adjusted by Jamone et al. (Jamone et al., 2016) to consider the physical grasping capabilities of the iCub hand, an anthropomorphic robotic hand applied by several research groups. This protocol offers a baseline to evaluate control algorithms on the iCub hand by assuming the human brain as the best possible controller.

The standardized performance tests proposed so far in the literature try to quantify dynamic and kinematic capabilities (finger and grasp forces, closing time, etc.) or very specific tasks, such as pick-and-place or pouring, among others. However, if we try to measure the anthropomorphism, it is necessary to replicate the most characteristic grasp types (GTs) in the human hand and to include a variety of objects used in activities of daily living (ADLs). In a previous work by the authors (Llop-Harillo and PérezGonzález, 2017) a preliminary protocol was proposed to test the grasping performance, including the most characteristic GTs and using different common objects. This protocol was used to obtain the coordination motion among the fingers of a new low-cost 3D-printed hand prototype, the IMMA hand. However, statistical validations are still needed for this work. Other 
studies seek to evaluate anthropomorphic prosthetic hands (Cipriani et al., 2008; Sun et al., 2014) and robotic hands (Konnaris et al., 2016; Tian et al., 2017) by applying the Cutkosky's Taxonomy (Cutkosky, 1989) or the GRASP Taxonomy proposed by Feix et al. (Feix et al., 2009). However, they do not use a common set of objects and are tailored to specific hand designs. Therefore, they do not present a repeatable or comparable index for measuring the grasp dexterity among arbitrary hands.

Pushing forward the approaches for benchmarking presented above, some indicatory questions arise: How to define a benchmark to evaluate the grasping capability of anthropomorphic artificial hands both for robotic and prosthetic applications? How to prove that an artificial hand is able to replicate the main types of human grasps? How to produce a benchmark easily replicable and able to compare different hand designs in order to foster future improvements of the grasping capabilities?

The objective of this paper is to establish a universal experimental benchmark to evaluate the ability of both robotic and prosthetic anthropomorphic hands to produce successful grasps in a human-like manner. The standardized protocol should be a functional test including the main GTs typical of human grasping in ADLs (Bullock et al., 2013; Feix et al., 2016; Light et al., 2002; Sollerman and Ejeskär, 1995; Vergara et al., 2014; Wang et al., 2018). Moreover, the objects used in the protocol should preferably be contained in a standardized set to enable the repeatability of the tests performed. In contrast to other standardized protocols such as SHAP (Light et al., 2002), the proposed protocol should evaluate both the functionality and the human-like execution of the different GTs, according to the human strategies. In addition, we aim to define a protocol, which can be used in different stages of the development cycle. Different alternatives for the mechanical design of the hand, the actuation method or the control algorithms should be comparable applying the proposed protocol.

\section{The Anthropomorphic Hand Assessment Protocol (AHAP)}

\subsection{Methodology}

The objective of this study is to propose a benchmark providing a reliable measure of the grasping ability of anthropomorphic hands. Grasping ability is understood here as the ability of the hand not only to effectively grasp a representative set of daily life objects, but also to maintain a stable grip under motion of the arm without external forces. We follow the terminology 


\section{Chapter 2}

proposed by Calli et al. (Calli et al., 2015) which defines a protocol as an experimental setup for a given manipulation task including the procedures to follow as well as a scoring scheme as a benchmark for the quantification of performance of the measured device or control algorithm. In order to define the benchmark, several steps were followed:

1. Selection of a representative set of grasp types (GTs) or grasp postures.

2. Selection of objects of different size, shape and weight, typically grasped with these GTs.

3. Definition of a preliminary protocol for testing the hand while grasping the selected objects.

4. Definition of a scoring system to obtain a numeric outcome measure from the test.

5. Test the preliminary protocol with different versions of an anthropomorphic robotic hand with distinct contact surface characteristics.

6. Statistical analysis of reliability, consistency and responsiveness of the protocol.

7. Modifications of the protocol and the scoring method in order to improve the reliability of the benchmark.

8. Test the improved protocol with the same robotic hand used in step 5 for the preliminary protocol.

9. Statistical analysis of reliability, consistency and responsiveness of this final protocol.

10.Application of the improved protocol to compare the grasping ability of two versions of an anthropomorphic prosthetic hand in order to validate the sensitivity of the protocol to fine-granular changes in the design of the hand.

The purpose of the protocol is to assess an anthropomorphic artificial hand's ability to firmly grasp a variety of objects and perform other manual tasks adopting different prototypical postures (GT), specified for each object/task and commonly used by the human hand in ADLs. The hand is operated by a human subject and the protocol is therefore applicable to prosthetic and robotic hands.

By applying this protocol, we obtain the following results:

- The total Grasping Ability Score (GAS) quantifying the proficiency of the hand to perform all the postures/tasks. 
- The partial GAS quantifying the proficiency to perform each specific posture/task.

- A qualitative impression of the advantages and disadvantages of the hand, its control method and its actuation device.

- A starting point to identify possible reasons for the failed grasps/tasks. The protocol allows an experimental identification of the difficulties in grasping and a classification of the limitations (e.g. finger orientation, friction between hand and object, finger coordination with the actuation device, feedback, force capability, etc.)

To be able to compare different artificial hands and to evaluate the possible influence of the actuation and control methods, the details of the tested setup including information on the artificial hand and the actuation device or actuation control used are also requested with the evaluation. A fair comparison of different artificial hand designs is possible if the same actuation or control method is used for all of them.

\subsection{Grasp types and objects}

The proposed protocol is divided in 26 tasks as shown in Table 1. It involves eight different GTs, coincident with those used in our previous study (Llop-Harillo and Pérez-González, 2017): pulp pinch (PP), lateral pinch (LP), diagonal volar grip (DVG), cylindrical grip (CG), extension grip (EG), tripod pinch (TP), spherical grip (SG) and hook grip (H). The selection was made based on the results of a previous field study about grasps applied in ADLs (Vergara et al., 2014) and on previous research in the area of human grasp analysis, prosthetics and rehabilitation (Bullock et al., 2013; Feix et al., 2016; Light et al., 2002; Sollerman and Ejeskär, 1995; Wang et al., 2018). The AHAP includes all the main GTs included in those works, accounting for more than $90 \%$ in grasp frequency. PP and TP account together for $29 \%-$ $48 \%$, LP for $9 \%-20 \%$, CG for $12 \%-25 \%$ and the rest of GTs for $18 \%-36 \%$.

We complemented our set of GTs with two non-grasping postures: platform (P) and index pointing/pressing (IP), given their importance for a multigrasp prosthetic hand (Varol et al., 2014).

Three different objects of the YCB set (Calli et al., 2015) have been selected for each GT in order to account for variations in size, shape, weight, texture and rigidity. This selection includes a representative subset of the possible variety of objects in ADLs within the limitation of objects available in the YCB set. For each non-grasping posture, one object of the YCB set 


\section{0 | Chapter 2}

was selected. In total the 25 objects shown in Table 1 are used for the benchmark. These objects include all different categories of the YCB set: 1) food items, 2) kitchen items, 3) tool items, 4) shape items and 5) task items. The main dimensions and weights of the objects can be found in (Calli et al., 2015).

Table 1. Grasp types and objects (YCB set) used in the protocol ( $T_{i}$ indicates the task order of the protocol).

\begin{tabular}{|c|c|c|c|}
\hline Grasp types (GTs) & & cts and tasks & \\
\hline $\operatorname{Hook}(\mathrm{H})$ & $\begin{array}{l}\text { Skillet lid } \\
\left(\mathrm{T}_{01}\right)\end{array}$ & $\begin{array}{c}\text { Pitcher base } \\
\left(\mathrm{T}_{10}\right)\end{array}$ & $\begin{array}{c}\text { Wood blocks with } \\
\text { rope }\left(T_{19}\right)\end{array}$ \\
\hline $\begin{array}{l}\text { Spherical } \\
\text { grip (SG) }\end{array}$ & $\begin{array}{l}\text { Plastic apple } \\
\left(\mathrm{T}_{02}\right)\end{array}$ & $\begin{array}{l}\text { Softball } \\
\left(\mathrm{T}_{11}\right)\end{array}$ & $\begin{array}{l}\text { Mini soccer ball } \\
\left(\mathrm{T}_{20}\right)\end{array}$ \\
\hline Tripod pinch (TP) & $\begin{array}{l}\text { Large marker } \\
\qquad\left(\mathrm{T}_{03}\right)\end{array}$ & $\begin{array}{l}\text { Tuna can } \\
\left(\mathrm{T}_{12}\right)\end{array}$ & $\begin{array}{l}\text { Golf ball } \\
\left(\mathrm{T}_{21}\right)\end{array}$ \\
\hline Extension grip (EG) & $\begin{array}{l}\text { Plate } \\
\left(\mathrm{T}_{04}\right)\end{array}$ & $\begin{array}{c}\text { Cracker box } \\
\left(\mathrm{T}_{13}\right)\end{array}$ & $\begin{array}{c}\text { Chocolate pudding } \\
\text { box }\left(\mathrm{T}_{22}\right)\end{array}$ \\
\hline Cylindrical grip (CG) & $\begin{array}{l}\text { Chips can } \\
\left(\mathrm{T}_{05}\right)\end{array}$ & $\begin{array}{l}\text { Coffee can } \\
\left(\mathrm{T}_{14}\right)\end{array}$ & $\begin{array}{c}\text { Power drill } \\
\left(\mathrm{T}_{23}\right)\end{array}$ \\
\hline
\end{tabular}


Diagonal volar grip (DVG)

Lateral pinch (LP)

Pulp pinch (PP)

Index pointing/pressing (IP)
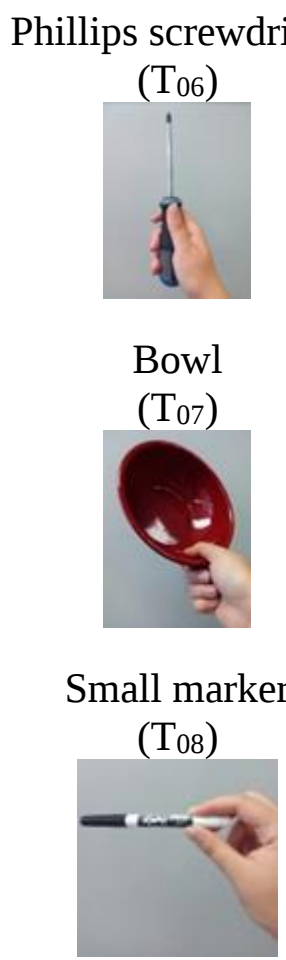

Spatula

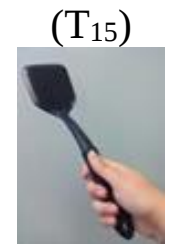

XS clamp

$\left(\mathrm{T}_{16}\right)$

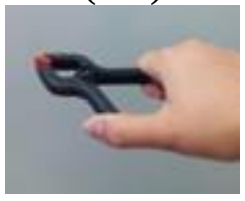

Plastic pear

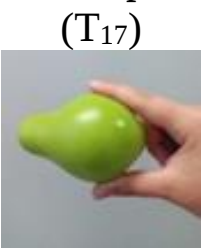

Timer $\left(\mathrm{T}_{09}\right)$

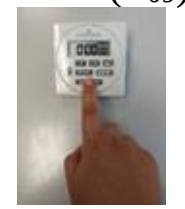

Plate $\left(\mathrm{T}_{18}\right)$

Platform (P)

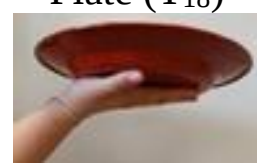

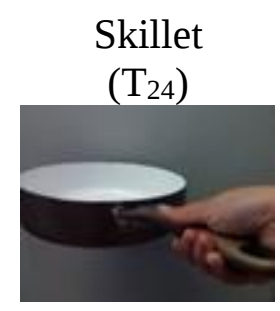

Key

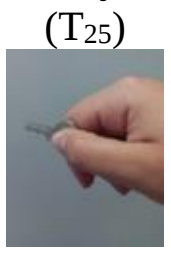

Washer $10 \mathrm{~mm}$

$\left(\mathrm{T}_{26}\right)$

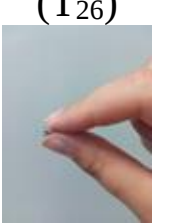

\subsection{Protocol and Benchmark}

The proposed protocol is applicable to anthropomorphic artificial hands which can be either robotic or prosthetic. The hand should be actuated by a human subject, either a disabled person wearing the prosthesis or an ablebodied person using an actuation or control device. For each task the objects are handed over to the subject by an operator holding them in the correct position for successful execution of the grasp. For different hand geometry, kinematics or control strategies, small variations in the orientation to present the object can be allowed, always pursuing the correct GT. Table 1 shows the approximate final position/orientation of the object with respect to the artificial hand in order to guide the operator and to increase the reproducibility. The operator releases the object once the grasp is performed by the artificial hand. The subject should be in a standing position during the 


\section{2 | Chapter 2}

test and located near a table. The subject will be instructed about the right grasping posture for each object/task and is allowed to practice with the object during a minute prior to the test. The correct GT is indicated by the operator and the subject should try to reproduce the demonstrated posture with the artificial hand as accurate as possible. Some damping material should be used on the floor and table near the subject to protect the objects in case of a grasp failure.

As explained above, several improvements were applied to the preliminary protocol according to an analysis of its reliability, consistency and responsiveness.

Table 2 shows the steps for the Anthropomorphic Hand Assessment Protocol (AHAP) and Table 3 explains its scoring system. The criteria for assessing GT correctness in the AHAP, taking into account some previous definition of the GTs (Sollerman and Ejeskär, 1995; Vergara et al., 2014), are listed in the appendix. The score of each grasp/task is provided by the operator. The test has a duration of approximately 80-100 min.

Scores for the three objects of each GT are added to obtain the final score for this grasping posture. Scores for all the objects/tasks are added to obtain the final score of the artificial hand. Normalized scores can be obtained by dividing by the maximum possible scores. Thus, the GAS can be expressed as a percentage of human grasping ability. The maximum GAS that an anthropomorphic artificial hand could achieve (100\%) corresponds to the healthy human hand. The minimum GAS of $0 \%$ describes an artificial hand unable to grasp any object.

Table 2. Steps of the Anthropomorphic Hand Assessment Protocol (AHAP) for each object.

\begin{tabular}{|c|c|}
\hline Step & Description \\
\hline 1 & $\begin{array}{l}\text { The operator shows the object and the correct grasping posture/task to the subject. } \\
\text { Detailed information about the posture/task for each object and the order to be } \\
\text { followed can be found in Table } 1 \text { (section } 2.2 \text { ). }\end{array}$ \\
\hline 2 & The operator helps the subject to practice the grasp/task for about one minute. \\
\hline 3 & $\begin{array}{l}\text { The operator hands the object over to the subject for the test. For index-pressing task } \\
\left(\mathrm{T}_{09}\right) \text { the timer is fixed to the table surface. }\end{array}$ \\
\hline 4 & $\begin{array}{l}\text { The subject actuates the artificial hand for grasping the object with the palm pointing } \\
\text { upwards. The operator releases the object as soon as the artificial hand has grasped } \\
\text { the object. The subject maintains the grasp for three seconds. For the index-pressing }\end{array}$ \\
\hline
\end{tabular}


task $\left(\mathrm{T}_{09}\right)$ the subject presses the button to start the timer and waits for three seconds. This step is followed immediately by step 5 and the sequence of steps $4-5$ is repeated three times.

5 While maintaining the grip, the subject rotates the hand in a natural way with low acceleration for the palm to point downwards $\left(180^{\circ}\right)$ and keeps the grip during three seconds in this position. For the index-pressing task $\left(\mathrm{T}_{09}\right)$ the subject presses the button again to stop the timer (maximum time to execute three seconds). For the platform position this step is not executed.

6 The subject releases the object, which is taken by the operator.

Table 3. Scoring system of the Anthropomorphic Hand Assessment Protocol (AHAP).

\begin{tabular}{|c|c|c|c|}
\hline Step & Task & $\begin{array}{l}\text { Score (for } \\
\text { each trial) }\end{array}$ & Scoring criteria \\
\hline \multirow[t]{3}{*}{4} & \multirow[t]{3}{*}{ All } & 1 & $\begin{array}{l}\text { The grasp is completed with the correct grasp type. } \\
\text { Detailed instructions to evaluate the grasping } \\
\text { posture can be found in the appendix. }\end{array}$ \\
\hline & & 0.5 & $\begin{array}{l}\text { The grasping posture is different to the one specified } \\
\text { in the appendix. }\end{array}$ \\
\hline & & 0 & The artificial hand cannot grasp the object. \\
\hline \multirow[t]{7}{*}{5} & \multirow[t]{3}{*}{$\begin{array}{l}\text { All the tasks } \\
\text { except } \mathrm{T}_{09} \text { and } \\
\mathrm{T}_{18}\end{array}$} & 1 & $\begin{array}{l}\text { No visible motion of the object with respect to the } \\
\text { hand is detected (for } \mathrm{T}_{19} \text { only the motion for the } \\
\text { portion of the rope located in the grasping area is } \\
\text { considered). }\end{array}$ \\
\hline & & 0.5 & $\begin{array}{l}\text { The object moves with respect to the hand but is not } \\
\text { dropped. }\end{array}$ \\
\hline & & 0 & The object is dropped. \\
\hline & \multirow[t]{3}{*}{$\mathrm{T}_{09}$} & 1 & Completed with the correct grasp type. \\
\hline & & 0.5 & $\begin{array}{l}\text { Completed with a grasp type different to the one } \\
\text { specified in the appendix. }\end{array}$ \\
\hline & & 0 & Not completed in less than three seconds. \\
\hline & $\mathrm{T}_{18}$ & - & Not additional point for this task. \\
\hline
\end{tabular}

The most significant changes of this improved protocol (AHAP) with respect to the preliminary version refer to the execution and scoring for step 


\section{4 | Chapter 2}

4. In the preliminary protocol this step was repeated for a maximum of three trials only in the event of failure in previous trials, with a decreasing score after each trial (1, 0.6, 0.4 points). The score given for a stable grasp with incorrect type was 0.2 points independent of the trial number it was achieved in. Moreover, in the preliminary protocol a detailed definition of requirements to fulfill for a GT to be considered correct (Appendix) was absent.

\section{Experimental evaluation}

\subsection{Tested Hands}

The presented protocol aims to evaluate the grasping ability of anthropomorphic artificial hands in robotic and prosthetic applications. We therefore validated it with a five-fingered robotic hand and a prosthesis to cover a wide range of use cases. These hand designs pursue different objectives in replication and augmentation of human grasping abilities. In the following paragraphs their individual specifications in design and control are described in detail.

\subsubsection{ARMAR-6 $v 1$ hand}

The ARMAR-6 v1 hand (ARMAR hand hereinafter), shown in Figure 1, is a prototypical robotic hand designed for the humanoid robot ARMAR-6 (Asfour et al., 2018). It is the first version of this hand, which has undergone significant design changes in the meantime.
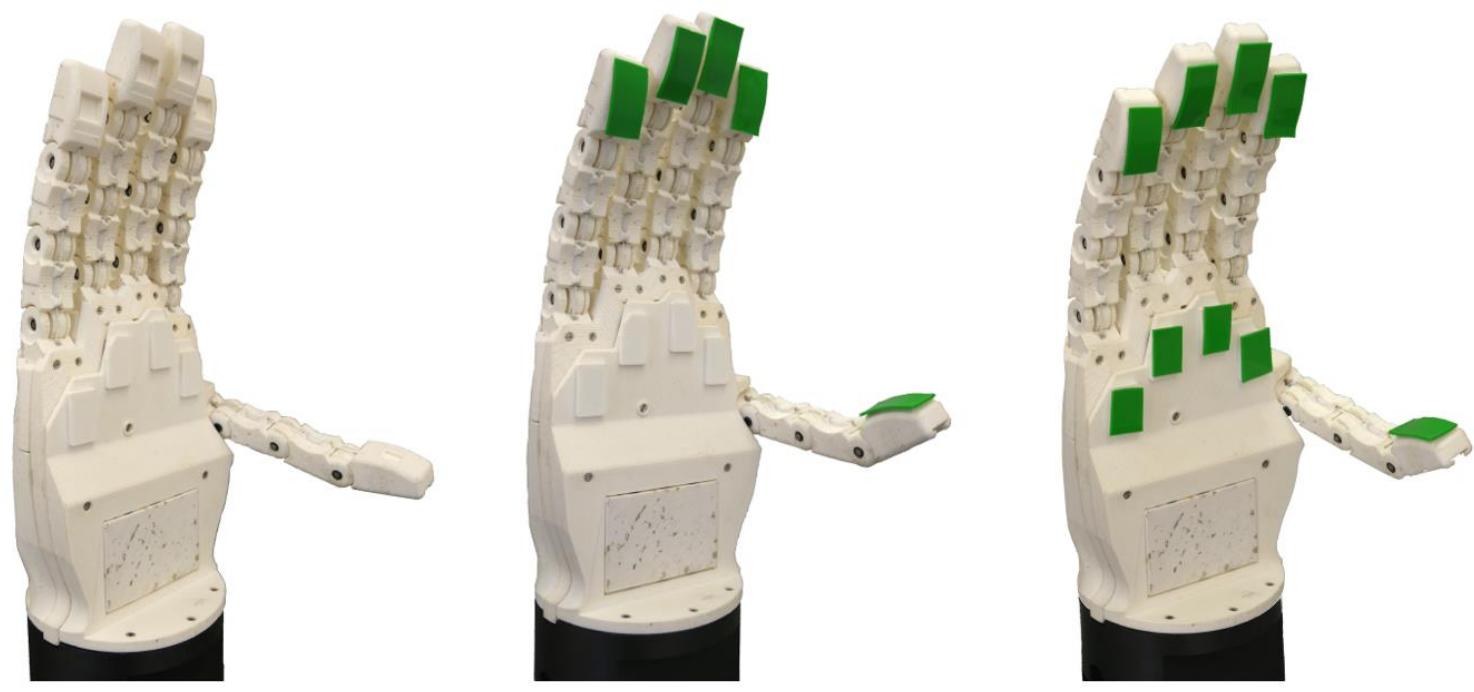

Figure 1. The three configurations of the ARMAR hand with different sets of friction pads; from left to right versions A1, A2 and A3. 
Altogether it has 15 degrees of freedom split up into three flexion joints per finger. Force transmission within the fingers is implemented with Dyneema tendon of $1 \mathrm{~mm}$ diameter. The hand is driven by two DC motors (1741U024CXR, Faulhaber GmbH) at $24 \mathrm{~V}$ with a 37:1 planetary gear (Faulhaber Series 17/1). While the thumb is actuated individually by one motor, all other fingers are actuated by a second motor via a force distributing lever mechanism adapted from the TUAT/Karlsruhe mechanism (Fukaya et al., 2013, 2000). It allows the fingers to close completely even when some of them are blocked, thereby enabling the hand to wrap around arbitrarily shaped objects. All finger joints are guided by sliding bearings and their passive reopening is ensured by extension springs. Although this movement is not supported by the springs, the finger mechanics are fully compliant and allow over-extension. This provides an inherent safety regarding self-collision and object contacts.

Matching to the size of the robot arm system, this hand is larger than the human model, as can be seen in Table 4.

Table 4. Sizing dimensions of the hands used for evaluation.

\begin{tabular}{lcc}
\hline Dimension (mm) & ARMAR hand & KIT Prosthetic Hand \\
\hline palm length & 144 & 111 \\
hand length (wrist to tip of the middle finger) & 253 & 189 \\
palm width & 100 & 87 \\
palm depth & 47 & 30 \\
\hline
\end{tabular}

All customized hand parts except for the lever of the mechanism are manufactured by fused deposition modeling from ABS plastic. The lever is made of high strength aluminum. The hand design includes pads amplifying the surface friction in fingers and palm. Throughout the experiments presented herein, the amount of applied friction pads was gradually increased including the bare plastic surface, five pads in the fingertips and an additional four pads in the palm as is shown in Figure 1. The pads are cut from an antislip foil (Kager Industrieprodukte $\mathrm{GmbH}$ ).

The hand is controlled with an Arduino board included in the palm. A serial interface including a comprehensive set of commands allows a simple velocity control of both motors as well as the approach of several dedicated finger positions. Control commands can be issued from any computational device providing serial communication. 


\section{6 | Chapter 2}

\subsubsection{KIT Prosthetic Hand}

The KIT Prosthetic Hand shown in Figure 2 is a 3D-printed prosthesis including an underactuated mechanism, sensors and an embedded control system (Weiner et al., 2018). It comprises 10 degrees of freedom with two flexion joints in each finger. The prosthesis is actuated by two DC motors (2224U012SR, Faulhaber GmbH) with an 86:1 transmission gear (Faulhaber Series 20/1R). It contains incremental encoders (Faulhaber IEH2-512). Similar to the ARMAR hand, a mechanical force distribution resembling the TUAT/Karlsruhe mechanism (Fukaya et al., 2013, 2000) is implemented to drive the four long fingers. Compared to the mechanism of the ARMAR hand, several improvements regarding the size and the amount of friction have been made.
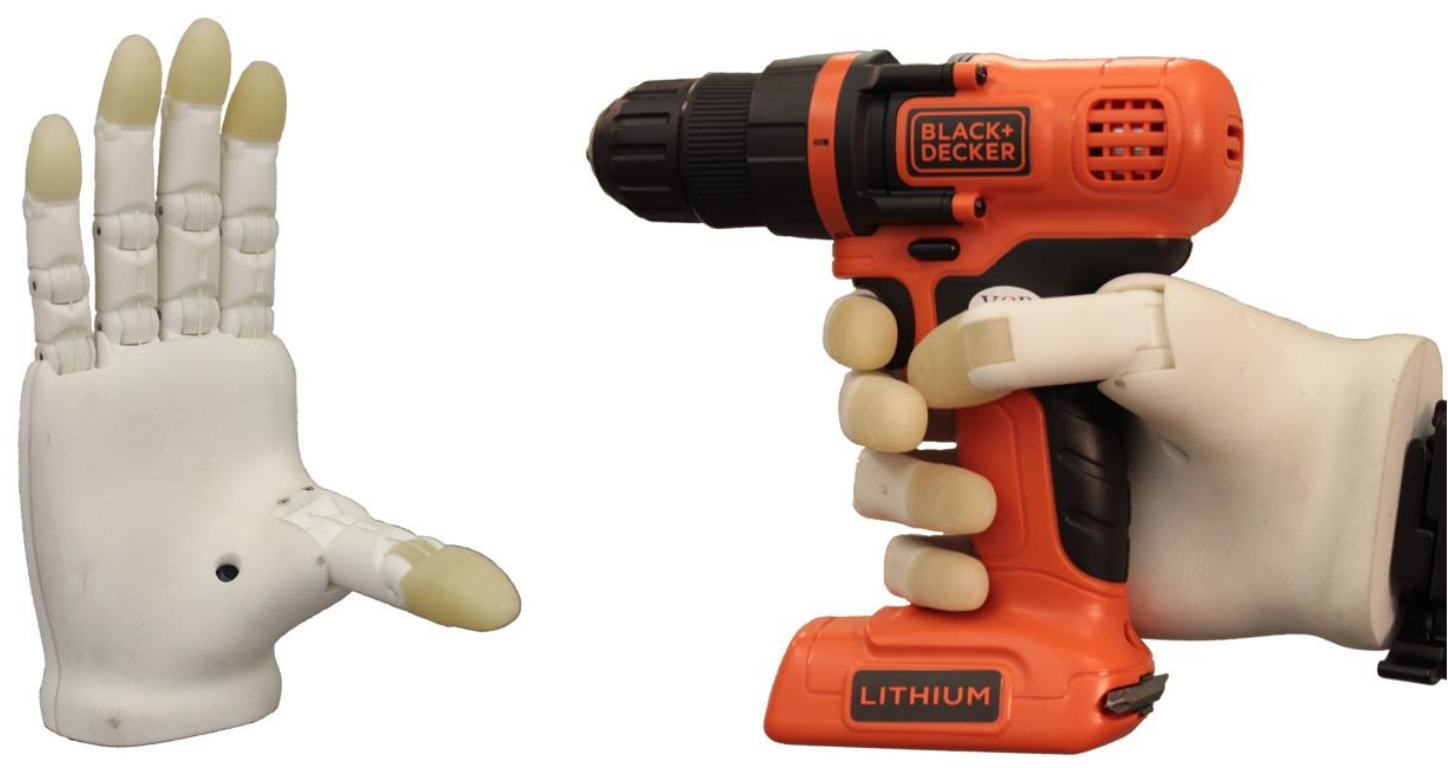

Figure 2. The final version of the KIT Prosthetic Hand (P2) fully opened (left) and while grasping the power drill (right).

The prosthesis is sized conforming a 50th percentile male human hand according to the German standard specification (DIN 33402-2). The resulting dimensions are noted in Table 4 . It has a fingertip force of up to $11.82 \mathrm{~N}$, a hook grasp force of $120 \mathrm{~N}$ and a hand closing time of $\sim 1.3 \mathrm{~s}$.

While the prosthetic hand supports various means of communication protocols, such as control including Bluetooth Low Energy and a direct serial interface, and control methods, we only employ a simple velocity control of the two motors by three buttons for the evaluations conducted herein.

Within this paper, we evaluate the final and published version of the KIT Prosthetic Hand (P2), depicted in Figure 2, and a preliminary version 
representing an earlier state of our development (P1). Both variants mainly differ in the placement of the mechanical parts within the finger joints and the lengths of the finger segments, which are not defined in the mentioned standard and were updated inspired by the human reference model of the Master Motor Map (Mandery et al., 2016) and studies from our previous work (Vergara et al., 2018).

\subsection{Benchmarking Tests}

To begin with, the preliminary protocol was executed with the three different configurations of the ARMAR hand with a gradually increasing number of friction pads attached to the hand as is shown in Figure 1. The first test performed with the preliminary protocol was on the hand without any pad (A1), the second was with five pads in the fingertips (A2) and for the third we applied four additional pads in the palm (A3).

Afterwards, these three configurations of the ARMAR hand were tested in the same order with the improved protocol (AHAP).

Finally, two versions of the KIT Prosthetic Hand (P1 and P2) were tested with the improved protocol (AHAP) in order to analyze the sensitivity to other changes in the design of a hand.

The motors of all the hands tested in this study were operated by different able-bodied subjects, members of the authors' research groups, with similar experience in using the hand to grasp objects, ensuring comparability between the tested anthropomorphic hand designs. The ARMAR hand was actuated from a laptop where velocity control commands were sent to the hand via a serial interface and the KIT Prosthetic Hand was controlled via a custom-made interface using velocity control with three buttons, one to close the four fingers, one to close the thumb and one to open all the fingers and thumb. The subject performing the test was responsible for actuating the motors in the best way to perform the grasp correctly, according to the different GTs. By using this method, the finger closing sequence and velocity are controlled by the human operating the hand. The hands' control did not include automatic motions based on preprogrammed grip patterns. As the hands evaluated are driven by an underactuated mechanism, the performed grip pattern is based on the object shape and the synergistic mechanical coupling of the adaptive fingers. 


\section{8 | Chapter 2}

\subsection{Validation of the benchmark}

The validity of the benchmark for measuring and comparing the grasping ability of anthropomorphic hands relies mainly on the following aspects, which are taken from psychometric outcome measures (Lindner et al., 2010):

- Intra- and inter-rater reliability: If the same hand is tested more than once by the same or by different raters, the results should be very similar.

- Internal consistency: The different tasks of the benchmark test should contribute to evaluate complementary aspects of the grasping ability without contradictory or inconsistent results.

- Responsiveness: The metric obtained from the benchmark is expected to vary under relevant changes of the grasping ability of the hand.

In order to evaluate these characteristics enabling also a comparison between the preliminary protocol and the improved one, several statistical analyses were conducted using SPSS statistical package (version 25, SPSS Inc, Chicago, USA).

In order to assess the inter-rater reliability, the tests were video-recorded and the videos were independently reviewed by five different raters from the authors' research groups to obtain the GAS.

Intra- and inter-rater reliability were assessed with the intraclass correlation coefficient (ICC) (Koo and Li, 2016). Inter-rater reliability was obtained for both the initial and improved protocols in order to get an indication of the improvement, whereas intra-rater reliability was only obtained for the improved protocol. Following the recommendations from Koo et al. (Koo and Li, 2016), inter-rater reliability was assessed with ICC based on a single-rated, absolute-agreement, two-way random-effects model. The data for each rater were the scores (from 0 to 2 for the initial protocol and from 0 to 6 for the improved protocol) corresponding to each of the 26 tasks and for all the 3 hand versions (26x3 cases). Intra-rater reliability for the improved protocol was assessed with ICC based on a single-rated, absolute-agreement, two-way mixed-effects model. For each of the three trials, the scores (from 0 to 2 ) corresponding to each of the 26 tasks for the 3 hand versions and for all the 5 raters were considered (26x3x5 cases). Values of ICC greater than 0.9 are considered as indicative of excellent reliability, values between 0.75 and 0.9 indicate good reliability and only moderate reliability can be claimed below 0.75 (Koo and Li, 2016).

The internal consistency of the benchmark to adequately reflect the grasping ability of the hands was assessed with the Cronbach's alpha through 
the scores for the different 26 tasks (from 0 to 2 for the initial protocol and from 0 to 6 for the improved protocol) corresponding to the three ARMAR hand versions for the five raters ( $3 \times 5=15$ cases). A good internal consistency is commonly considered if Cronbach's alpha is above 0.8 , whereas it can be considered excellent above 0.9 .

The responsiveness of the benchmark under changes in the hand design was assessed with the mean and standard deviation of the GAS obtained by each hand model. A significant difference in the means as compared to the standard deviation under several repetitions or raters is indicative of a good responsiveness. To quantify the responsiveness with a standard measure we used the standardized response mean (SRM) (Husted et al., 2000) of different hand model pairs. We computed SRM for a pair of hands by dividing the mean difference across raters of the GAS for those hands by the standard deviation of these differences. SRM is a standardized non-dimensional value. A value greater than 0.8 is considered indicative of a high responsiveness (Husted et al., 2000). Different values of SRM were obtained comparing firstly each combination of two versions of the ARMAR and secondly the two versions of the KIT Prosthetic Hand.

\section{Results}

\subsection{Grasping Ability Score (GAS)}

Mean value and standard deviation across raters of the GAS of the different hands using the protocols presented above, evaluated independently by five different raters according to the videos of the tests, are depicted in Figure 3. It shows the results for the three versions of the ARMAR hand (A1, A2 and A3) using the preliminary protocol and those for these three versions of the ARMAR hand and the two versions of the KIT Prosthetic Hand (P1 and P2) using the improved protocol (AHAP).

The results reflect the expected improvement in the GAS in both hands with the changes in the design (from A1 to A3 for ARMAR hand and from P1 to P2 for KIT Prosthetic Hand).

Moreover, the results highlight a significant reduction of the standard deviation across raters with the AHAP (ranging between 1.19\% - 2.15\%) with respect to the preliminary protocol $(9.14 \%-10.20 \%)$.

The GAS involves both the ability to replicate the human-like GTs and the effectiveness for maintaining these grasps under motion. Table 5 shows 


\section{0 | Chapter 2}

the normalized score obtained by each hand for both parts of the task (grasping and maintaining).

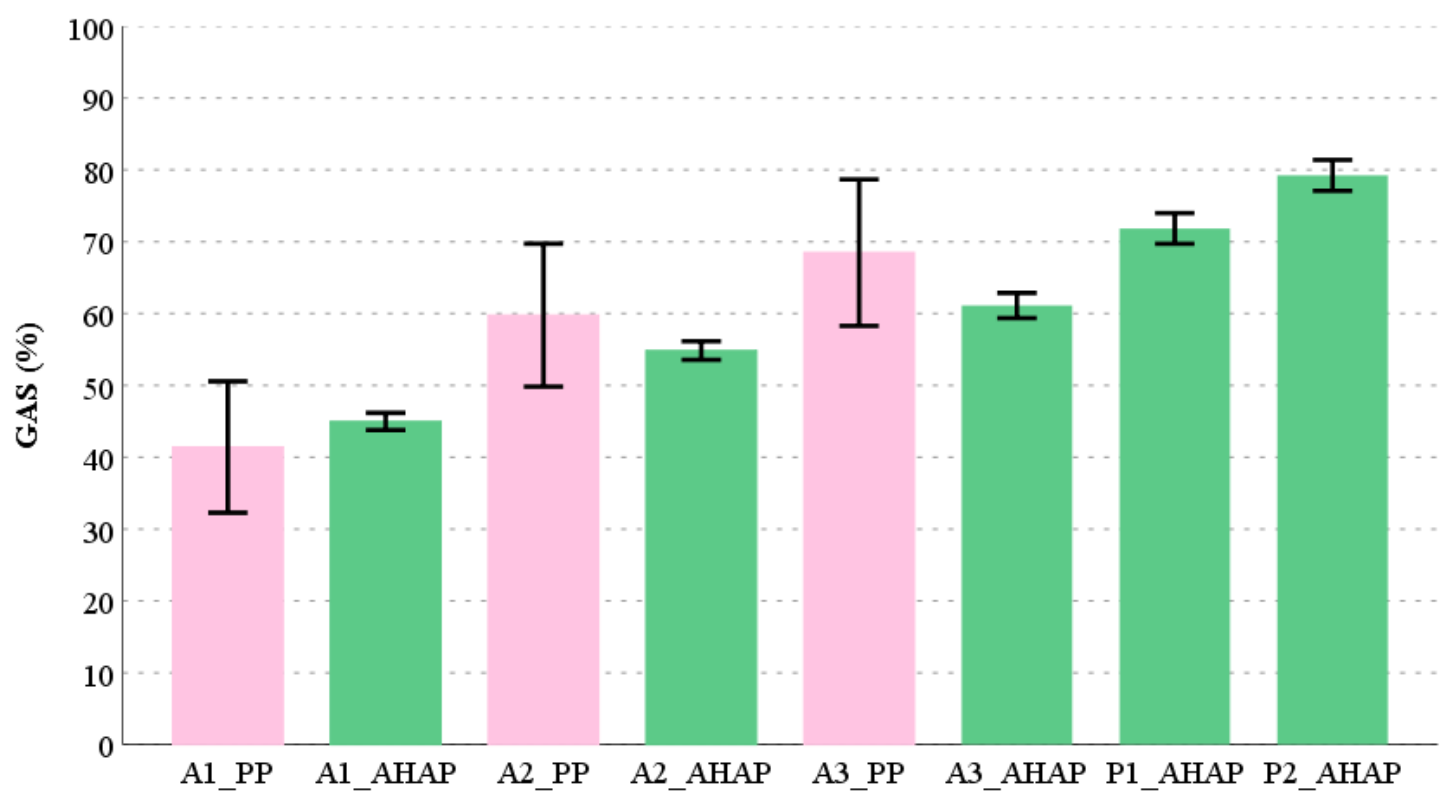

Figure 3. Mean value and standard deviation across raters of the GAS with the preliminary protocol (PP) and with the improved protocol (AHAP) for each hand model (A1: ARMAR-6 v1 robotic hand without any pad, A2: ARMAR-6 v1 robotic hand with five pads in the fingertips, A3: ARMAR-6 v1 robotic hand with five pads in the fingertips and four pads in the palm, P1: preliminary version of the KIT Prosthetic Hand, P2: final and published version of the KIT Prosthetic Hand).

Table 5. Mean GAS and mean score for each part of the task (grasping and maintaining) for each hand model with the AHAP.

\begin{tabular}{cccc}
\hline Hand & Grasping & Maintaining & GAS \\
\hline A1 & $52 \%$ & $37 \%$ & $45 \%$ \\
A2 & $59 \%$ & $50 \%$ & $55 \%$ \\
A3 & $62 \%$ & $60 \%$ & $61 \%$ \\
P1 & $65 \%$ & $79 \%$ & $72 \%$ \\
P2 & $68 \%$ & $91 \%$ & $79 \%$ \\
\hline
\end{tabular}

In addition, Figure 4 shows an analysis of the partial GAS for each GT (Table 1) obtained by the different tested hand models. This analysis could be interesting, for example for cases where the artificial hands have a specific purpose and the reproduction of some, but not all of the GTs is important. Index pointing (IP) and hook grasp (H) obtain the highest partial GAS for both hand types. The platform (P) posture was properly obtained with the ARMAR hand but not with the KIT Prosthetic Hand owing to the difference in hyperextension capabilities of both thumb designs. Pulp pinch (PP) and spherical grip (SG) are among the GTs with a higher scattering in the partial 
GAS depending on the hand design, as slight changes of the friction conditions have a high impact on the success of these GTs. A significant difference can be observed in the partial GAS obtained by the ARMAR hand configurations and the KIT Prosthetic Hand versions for extension grip (EG) and especially for cylindrical grip (CG) due to the achievable grasp force.

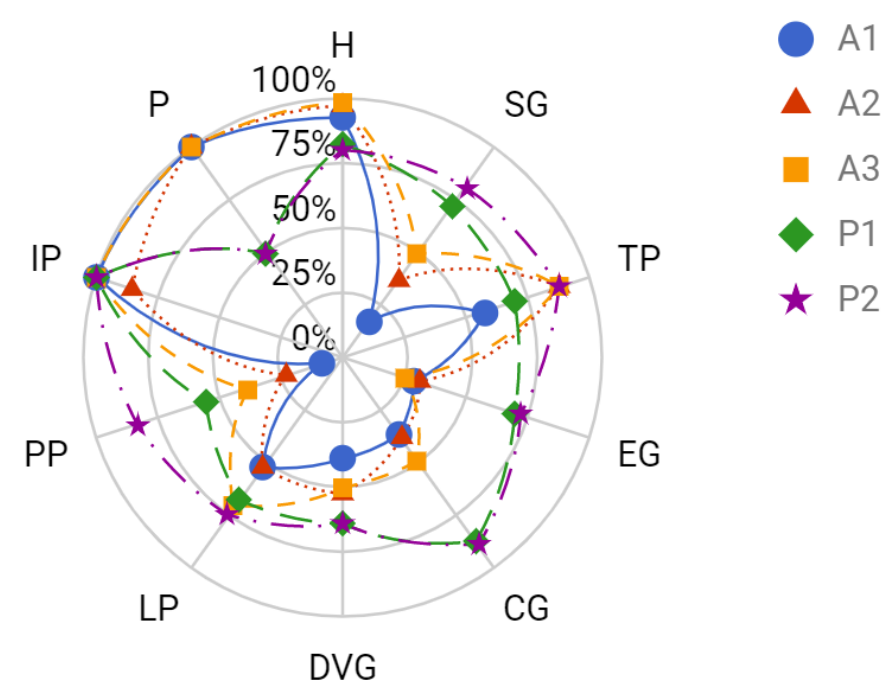

Figure 4. Mean Grasping Ability Score (GAS) grouped by grasp type (GT, Table 1) obtained for each hand model with the Improved Protocol (AHAP). H: hook, SG: spherical grip, TP: tripod pinch, EG: extension grip, CG: cylindrical grip,

DVG: diagonal volar grip, LP: lateral pinch, PP: pulp pinch, IP: index pointing/pressing, P: platform. A1: ARMAR-6 v1 robotic hand without any pad, A2: ARMAR-6 v1 robotic hand with five pads in the fingertips, A3: ARMAR-6 v1 robotic hand with five pads in the fingertips and four pads in the palm, P1: preliminary version of the KIT Prosthetic Hand, P2: final and published version of the KIT Prosthetic Hand.

\subsection{Validation of the benchmark}

Table 6 shows the results of the statistical analysis undertaken to evaluate the validity of the benchmark for quantitatively measuring and comparing the grasping ability of anthropomorphic hands. Additionally, the responsiveness can also be assessed in Figure 3.

The inter-rater reliability was clearly improved by the adaptations in the protocol from moderate/good for the preliminary protocol to excellent (greater than 0.9) for the improved protocol (AHAP). In addition, the results of the improved protocol indicate a good intra-rater reliability (above 0.8). The internal consistency of the test is good, with a Cronbach's alpha between 0.8 and 0.9 , and very similar for both protocols. This similarity seems reasonable provided that the items of the test (the different tasks) are the 


\section{2 | Chapter 2}

same in both cases. The responsiveness of the test to changes in the hand design is considered high, with values clearly above 0.8 for the SRM for all the comparisons of hand pairs in both the preliminary and improved protocols.

Table 6. Results of the validation of the benchmark.

\begin{tabular}{|c|c|c|c|c|c|}
\hline Validation aspects & $\begin{array}{l}\text { Statistical } \\
\text { parameters }\end{array}$ & \multicolumn{2}{|c|}{ Preliminary Protocol } & \multicolumn{2}{|c|}{$\begin{array}{l}\text { Improved Protocol } \\
\text { (AHAP) }\end{array}$} \\
\hline $\begin{array}{l}\text { Inter-rater reliability } \\
\text { [95\% confidence } \\
\text { interval] }\end{array}$ & ICC & \multicolumn{2}{|c|}{$0.771[0.670-0.846]$} & \multicolumn{2}{|c|}{$0.969[0.957-0.978]$} \\
\hline $\begin{array}{l}\text { Intra-rater reliability } \\
\text { [95\% confidence } \\
\text { interval] }\end{array}$ & ICC & \multicolumn{2}{|c|}{ - } & \multicolumn{2}{|c|}{0.839 [0.813-0.863] } \\
\hline Internal consistency & Cronbach's alpha & \multicolumn{2}{|c|}{0.897} & \multicolumn{2}{|c|}{0.846} \\
\hline \multirow[t]{4}{*}{ Responsiveness } & SRM & A1-A2 & 2.4 & A1-A2 & 8.2 \\
\hline & & A2-A3 & 4.4 & A2-A3 & 5.7 \\
\hline & & A1-A3 & 3.4 & A1-A3 & 21.5 \\
\hline & & - & - & P1-P2 & 7.8 \\
\hline
\end{tabular}

\subsection{Qualitative impressions from the grasp trials}

The protocol allows to record additional subjective results from the observations made during the grasp trials. These should be annotated by the operator and/or the subject. Although this information is not part of the quantitative comparison provided by the benchmark, it offers an additional possibility to note and discuss findings and insights gained throughout the grasping process, which can be helpful for later design improvements.

To demonstrate the merit of such comments for the further development of tested prototypes, some exemplary cases from the evaluations presented in this study are discussed in detail.

The surface properties of the hand parts in contact with the object are prominently reflected in grasp stability. While especially the influence of friction is well known and quantified in robotic simulations, the presented protocol is able to corroborate this coherence and numerically prove the 
benefit even with small improvements of the hand's characteristics. During the first evaluation of the ARMAR hand without any pads, the problem of objects slipping out of a grasp was clearly notable in seven objects. Amongst others especially the plastic fruits, the small marker and the tuna can were affected and could not be grasped at all. According to this observation, the hand was gradually equipped with high friction pads, as described in section 3.1.1. As a result, the grasping performance improved notably with all mentioned objects being successfully grasped at least once.

Especially for heavy objects and those with an uneven distribution of mass, a high force is critical for grasp success. This can be directly noted in the results with the ARMAR hand. Also with the pads on fingertips and palm applied, it has still difficulties with grasping the plate, the coffee can, the power drill and the skillet. Due to its improved motor control and reduced friction in the transmission, the KIT Prosthetic Hand is designed to be strikingly stronger than the ARMAR hand, which is reflected in the grasping results of the respective objects. While the latter is not able to hold the grip with the palm pointing downwards with any of those objects, the KIT Prosthetic Hand is able to perform a successful hand turning motion at least twice for all of them.

The influence of finger kinematics on the grasp quality is more difficult to identify. However, the evaluations of the two versions of the KIT Prosthetic Hand prove its visibility in the presented protocol. The original dimensioning of the finger phalanges included rather long proximal and short distal finger segments. In addition, the distal interphalangeal joints are fixed at an angle of $20^{\circ}$ for both prosthetic designs. The fingertips were therefore unable to touch the palm, as the total length of intermediate and distal finger phalanges was too short compared to the proximal phalanx. This caused motion when rotating objects held with a hook grasp and complicated the grasping of thin objects like the markers. For the second version the lengths were updated according to a thorough study of human kinematics. By these means the grasping behavior could be improved for both markers and the wood blocks with a rope.

The relevance of the thumb opposition has attracted attention during the tests using this benchmark. The thumb opposition is influenced by both the orientation of the thumb and the abduction/adduction degree of freedom. Limitations in this aspect imply difficulties to correctly reproduce the diagonal volar grip and the lateral pinch GTs. 


\section{4 | Chapter 2}

\section{Discussion}

Latest reviews on benchmarks and testing methods in the field of prosthetics (Mio et al., 2018) and robotics (Quispe et al., 2018) emphasize the relevance of assessing artificial hand functional abilities with standardized testing methodologies. In most of the previous studies assessing artificial hand prototypes some preliminary grasping tests are included, but the comparability among research groups is very limited as they differ in the used objects and evaluation metrics.

In this study we propose an experimental protocol and benchmark applicable to both robotic and prosthetic anthropomorphic hands: the Anthropomorphic Hand Assessment Protocol (AHAP). The AHAP defines a total Grasping Ability Score (GAS) that quantifies numerically the capability of performing everyday grasps. This benchmark does not only provide a basis of comparison, but also a way to recognize possible improvements to the designs of the hands analyzed. The AHAP is inspired by a proposed preliminary protocol (Llop-Harillo and Pérez-González, 2017) with major improvements based on the statistical validation. We analyzed the intra- and inter-rater reliability, internal consistency and responsiveness (Table 6) verifying the robustness of the AHAP across raters and proving the comparability of the results also across different hands and testing conditions. The improvements were additionally demonstrated by a significant decrease of the standard deviation of the GAS evaluated by five different raters (Figure 3). The variations between the results of the GAS for different configurations of the hands are higher than the standard deviation. It can therefore be concluded that the proposed benchmark is a powerful tool for evaluating and comparing different artificial hand designs.

The AHAP uses the YCB set of objects proposed by Calli et al. (Calli et al., 2015). The use of an internationally available set of objects facilitates the comparison among hand developers. We developed the protocol according to the recommendations included in (Calli et al., 2015) where some examples are proposed. Unlike the protocols included in that study, the AHAP covers a wide range of aspects of the manipulation problem using objects of different sizes, shapes, weights, textures and rigidities from all different categories of the YCB set (food, kitchen, tool, shape and task items). The GAS obtained from the AHAP considers the ability to produce correctly each important human GT, and also the ability to maintain it under motion of the arm. It is evident that forces acting on the object during the execution of real tasks can be higher than those acting when turning the hand with the grasped 
object, but the objective of the AHAP is not to obtain the force limits for each GT. The objective is more focused on the ability to reproduce the different human GTs, which mainly depends on the kinematic structure of the hand, its control scheme, and the properties of the materials in the contact areas, in particular stiffness and roughness. The force limits are also affected by these parameters, but these limits depends also on the dynamic capability of the actuators and they should be investigated with specific protocols and metrics. We hypothesize that separating the different design aspects in the evaluation can be more efficient for improving artificial hands design. Nevertheless, in the AHAP the force limits are implicitly considered through the use of objects of different weights ranging from 0.7 to $950 \mathrm{~g}$.

A common task both in robotics and prosthetics is to pick up an object from a table in a random position. However, in the proposed protocol we did not want to include the additional difficulties related to environmental grasp constraints and collision-free motion planning that arise if the hand is connected to a robotic arm or an able-bodied adaptor for a human operator. We instead focused the protocol on the ability of the hand for firmly grasping, using human-like GTs, when the object is presented in the correct position for the success in the grasp. By including simple grasping tasks, we avoid adverse influences possibly arising from complex tasks. We thereby provide a simple, transparent method to evaluate prehension. In addition, the benchmark tries to evaluate the anthropomorphism of the artificial hands based on their capability to perform the most frequently used human GTs in ADLs. Obtaining the highest GAS should be desirable for any anthropomorphic hand, taking into account that both humanoid robot's hands and prosthetic hands should behave human-like. Nevertheless, some specialized robotic hands may have specific requirements for their applications shifting importance to some of the GTs presented in the AHAP. In those cases, the results of the GAS can be individually evaluated for each GT (Figure 4), allowing a detailed and precise representation of the hand's abilities.

It is worth to note that the AHAP goes beyond previous studies (Cipriani et al., 2008; Jamone et al., 2016) by evaluating the grasping ability of artificial anthropomorphic hands independently of their actuation or control system. However, a fair comparison of one of the main aspects such as the mechanical design of the hands, the actuation method or the control algorithms, is only possible when the other ones are fixed. 


\section{6 | Chapter 2}

In this study we applied the AHAP to evaluate three different configurations (A1, A2, A3) of the ARMAR hand (a robotic hand from KIT) and two versions of the KIT Prosthetic Hand (P1, P2). The results show that the mean GAS increased from A1 to A3 due to the addition of friction pads, shifting from a value of $45 \%$ for A1 to $55 \%$ for A2 and $61 \%$ for A3. Furthermore, the improvement of the mean GAS from P1 (72\%) to P2 (79\%) proves the effectiveness of the improvement in the finger kinematics of the last version of the KIT Prosthetic Hand.

The proposed GAS assesses both the ability to replicate the human-like GTs and the effectivity of these grasps maintaining them under motion. It thereby evaluates the anthropomorphism and the functionality of robotic and prosthetic hands. For the hands tested, as shown in Table 5, the A3 has a similar score for replicating the GTs (62\%) as for maintaining them (60\%), while the P2 has a comparable score for replicating the GTs (68\%) but a significantly higher score for maintaining them (91\%). That means that the better GAS of the final version of the KIT Prosthetic Hand compared to the last configuration of the ARMAR hand is caused by the higher ability to maintain a grasp under motion. This result is due to an improvement in the grasping force, applying considerably stronger motors, a more intuitive control and reduced friction in the transmission.

According to the definition of Falco et al. (Falco et al., 2015) the AHAP is considered a functional test and could complement some other component and system tests proposed in the literature (Falco et al., 2015; Liu et al., 2015; Mio et al., 2018; Quispe et al., 2018). Additionally, it could be interesting to compare the assessment of different anthropomorphic hands using the AHAP and with analytical metrics proposed in the literature that give an index of the anthropomorphism of artificial hands (Feix et al., 2013; Liarokapis et al., 2013).

\section{Conclusions}

In this paper, we present a new experimental, standardized and reproducible benchmark that has been statistically validated. We propose an evaluation protocol (AHAP) that includes the most frequently used GTs in ADLs performed by humans and a wide range of objects included in an internationally available object set (YCB set). With the proposed benchmark a reliable measure of the grasping ability of anthropomorphic robotic and prosthetic hands can be obtained, evaluating the functionality and anthropomorphism of the achieved grasps. In a thorough study we analyzed 
the GAS of three configurations of a robotic hand (ARMAR hand) and two versions of the KIT Prosthetic Hand. We offer a validated tool to evaluate and compare the different aspects of artificial hands: the mechanical design, the actuation system and the control strategy. The results obtained with the benchmark could be used for comparison of several hand designs but also to foster future improvements of their grasping capabilities. We used the benchmark to demonstrate the improvements of the tested hands in aspects as the grasping surface, the grasp force and the finger kinematics. Nevertheless, in future works the AHAP could be used to compare different force transmission systems such as tendons and bar linkages or different control strategies.

\section{Acknowledgments}

This work was supported by the Spanish Ministry of Economy and Competitiveness and ESF [grant number BES-2015-076005]; Spanish Ministry of Economy and Competitiveness and ERDF [grant numbers DPI2014-60635-R, DPI2017-89910-R]; Universitat Jaume I [grant number UJI-B2017-70] and the German Federal Ministry of Education and Research (BMBF) under the project INOPRO (16SV7665).

\section{References}

Asfour, T., Kaul, L., Wachter, M., Ottenhaus, S., Weiner, P., Rader, S., Grimm, R., Zhou, Y., Grotz, M., Paus, F., Shingarey, D., Haubert, H., 2018. ARMAR6: A Collaborative Humanoid Robot for Industrial Environments, in: 2018 IEEE-RAS 18th International Conference on Humanoid Robots (Humanoids). IEEE, pp. 447-454.

Belter, J.T., Leddy, M.T., Gemmell, K.D., Dollar, A.M., 2016. Comparative clinical evaluation of the Yale Multigrasp Hand, in: 2016 6th IEEE International Conference on Biomedical Robotics and Biomechatronics (BioRob). IEEE, pp. 528-535.

Belter, J.T., Segil, J.L., Dollar, A.M., Weir, R.F., 2013. Mechanical design and performance specifications of anthropomorphic prosthetic hands: A review. The Journal of Rehabilitation Research and Development, 50(5), 599.

Bonsignorio, F., Del Pobil, A.P.D.P., Messina, E., 2014. Fostering Progress in Performance Evaluation and Benchmarking of Robotic and Automation Systems. IEEE Robotics \& Automation Magazine, 21(1), 22-25.

Bostelman, R., Messina, E., Foufou, S., 2017. Cross-industry standard test method developments: from manufacturing to wearable robots. Frontiers of 


\section{8 | Chapter 2}

Information Technology \& Electronic Engineering, 18(10), 1447-1457.

Bullock, I.M., Zheng, J.Z., Rosa, S.D. La, Guertler, C., Dollar, A.M., 2013. Grasp Frequency and Usage in Daily Household and Machine Shop Tasks. IEEE Transactions on Haptics, 6(3), 296-308.

Calli, B., Walsman, A., Singh, A., Srinivasa, S., Abbeel, P., Dollar, A.M., 2015. Benchmarking in Manipulation Research: Using the Yale-CMU-Berkeley Object and Model Set. IEEE Robotics \& Automation Magazine, 22(3), 36-52.

Cipriani, C., Zaccone, F., Micera, S., Carrozza, M.C., 2008. On the Shared Control of an EMG-Controlled Prosthetic Hand: Analysis of User-Prosthesis Interaction. IEEE Transactions on Robotics, 24(1), 170-184.

Controzzi, M., Cipriani, C., Carrozza, M.C., 2014. Design of Artificial Hands: A Review, in: Springer Tracts in Advanced Robotics. pp. 219-246.

Cutkosky, M.R., 1989. On grasp choice, grasp models, and the design of hands for manufacturing tasks. IEEE Transactions on Robotics and Automation, 5(3), 269-279.

Duong, T., Wagner, B., Abraham, T., Davidson, M., Bains, G., Daher, N., Friedrich, A., 2017. Comparative Study of Functional Grasp and Efficiency Between a 3D-Printed and Commercial Myoelectric Transradial Prosthesis Using Able-Bodied Subjects. Journal of Prosthetics and Orthotics, 29(3), 112-118.

Falco, J., Van Wyk, K., Liu, S., Carpin, S., 2015. Grasping the Performance: Facilitating Replicable Performance Measures via Benchmarking and Standardized Methodologies. IEEE Robotics \& Automation Magazine, 22(4), 125-136.

Feix, T., Pawlik, R., Schmiedmayer, H.-B., Romero, J., Kragic, D., 2009. A comprehensive grasp taxonomy. Robotics, Scince And Systems Conference: Wokshop On Understanding The Human Hand For Advancing Robotic Manipulation.

Feix, T., Romero, J., Ek, C.H., Schmiedmayer, H.-B., Kragic, D., 2013. A Metric for Comparing the Anthropomorphic Motion Capability of Artificial Hands. IEEE Transactions on Robotics, 29(1), 82-93.

Feix, T., Romero, J., Schmiedmayer, H.-B., Dollar, A.M., Kragic, D., 2016. The GRASP Taxonomy of Human Grasp Types. IEEE Transactions on HumanMachine Systems, 46(1), 66-77.

Fukaya, N., Asfour, T., Dillmann, R., Toyama, S., 2013. Development of a fivefinger dexterous hand without feedback control: The TUAT/Karlsruhe humanoid hand, in: 2013 IEEE/RSJ International Conference on Intelligent Robots and Systems. IEEE, pp. 4533-4540.

Fukaya, N., Toyama, S., Asfour, T., Dillmann, R., 2000. Design of the TUAT/Karlsruhe humanoid hand, in: Proceedings. 2000 IEEE/RSJ 
International Conference on Intelligent Robots and Systems (IROS 2000) (Cat. No.00CH37113). IEEE, pp. 1754-1759.

Husted, J.A., Cook, R.J., Farewell, V.T., Gladman, D.D., 2000. Methods for assessing responsiveness: A critical review and recommendations. Journal of Clinical Epidemiology, 53(5), 459-468.

Jamone, L., Bernardino, A., Santos-Victor, J., 2016. Benchmarking the Grasping Capabilities of the iCub Hand With the YCB Object and Model Set. IEEE Robotics and Automation Letters, 1(1), 288-294.

Kasper, A., Xue, Z., Dillmann, R., 2012. The KIT object models database: An object model database for object recognition, localization and manipulation in service robotics. The International Journal of Robotics Research, 31(8), 927934.

Kemp, C., Edsinger, A., Torres-Jara, E., 2007. Challenges for robot manipulation in human environments [Grand Challenges of Robotics]. IEEE Robotics \& Automation Magazine, 14(1), 20-29.

Konnaris, C., Gavriel, C., Thomik, A.A.C., Faisal, A.A., 2016. EthoHand: A dexterous robotic hand with ball-joint thumb enables complex in-hand object manipulation, in: 2016 6th IEEE International Conference on Biomedical Robotics and Biomechatronics (BioRob). IEEE, pp. 1154-1159.

Koo, T.K., Li, M.Y., 2016. A Guideline of Selecting and Reporting Intraclass Correlation Coefficients for Reliability Research. Journal of Chiropractic Medicine, 15(2), 155-163.

Kragten, G.A., Meijneke, C., Herder, J.L., 2010. A proposal for benchmark tests for underactuated or compliant hands. Mechanical Sciences, 1(1), 13-18.

Krumlinde-Sundholm, L., Holmefur, M., Kottorp, A., Eliasson, A.-C., 2007. The Assisting Hand Assessment: current evidence of validity, reliability, and responsiveness to change. Developmental Medicine \& Child Neurology, 49(4), 259-264.

Liarokapis, M. V., Artemiadis, P.K., Kyriakopoulos, K.J., 2013. Quantifying anthropomorphism of robot hands, in: 2013 IEEE International Conference on Robotics and Automation. IEEE, pp. 2041-2046.

Liarokapis, M. V., Artemiadis, P.K., Kyriakopoulos, K.J., 2012. Functional Anthropomorphism for human to robot motion mapping, in: 2012 IEEE ROMAN: The 21st IEEE International Symposium on Robot and Human Interactive Communication. IEEE, pp. 31-36.

Light, C.M., Chappell, P.H., Kyberd, P.J., 2002. Establishing a standardized clinical assessment tool of pathologic and prosthetic hand function: Normative data, reliability, and validity. Archives of Physical Medicine and Rehabilitation, 83(6), 776-783.

Lindner, H.Y.N., Nätterlund, B.S., Hermansson, L.M.N., 2010. Upper Limb 
Prosthetic Outcome Measures: Review and Content Comparison Based on International Classification of Functioning, Disability and Health. Prosthetics and Orthotics International, 34(2), 109-128.

Liu, Y., Yang, D., Jiang, L., Liu, H., 2015. A synthetic framework for evaluating the anthropomorphic characteristics of prosthetic hands, in: 2015 IEEE International Conference on Advanced Intelligent Mechatronics (AIM). IEEE, pp. 877-884.

Llop-Harillo, I., Pérez-González, A., 2017. System for the experimental evaluation of anthropomorphic hands. Application to a new 3D-printed prosthetic hand prototype. International Biomechanics, 4(2), 50-59.

Mandery, C., Terlemez, O., Do, M., Vahrenkamp, N., Asfour, T., 2016. Unifying Representations and Large-Scale Whole-Body Motion Databases for Studying Human Motion. IEEE Transactions on Robotics, 32(4), 796-809.

Mathiowetz, V., Volland, G., Kashman, N., Weber, K., 1985. Adult Norms for the Box and Block Test of Manual Dexterity. American Journal of Occupational Therapy, 39(6), 386-391.

Mio, R., Sanchez, M., Valverde, Q., 2018. Mechanical Testing Methods for BodyPowered Upper-Limb Prostheses: A Review, in: 2018 IEEE 18th International Conference on Bioinformatics and Bioengineering (BIBE). IEEE, pp. 170176.

Puig, J.E.P., Rodriguez, N.E.N., Ceccarelli, M., 2008. A Methodology for the Design of Robotic Hands with Multiple Fingers. International Journal of Advanced Robotic Systems, 5(2), 22.

Quispe, A.H., Amor, H. Ben, Christensen, H.I., 2018. A Taxonomy of Benchmark Tasks for Robot Manipulation, in: Springer Proceedings in Advanced Robotics. pp. 405-421.

Singh, A., Sha, J., Narayan, K.S., Achim, T., Abbeel, P., 2014. BigBIRD: A largescale 3D database of object instances, in: 2014 IEEE International Conference on Robotics and Automation (ICRA). IEEE, pp. 509-516.

Sollerman, C., Ejeskär, A., 1995. Sollerman Hand Function Test: A Standardised Method and its Use in Tetraplegic Patients. Scandinavian Journal of Plastic and Reconstructive Surgery and Hand Surgery, 29(2), 167-176.

Stern, E.B., 1992. Stability of the Jebsen-Taylor Hand Function Test across three test sessions. The American journal of occupational therapy.: official publication of the American Occupational Therapy Association.

Sun, B., Xiong, C., Chen, W., Zhang, Q., Mao, L., Zhang, Q., 2014. A novel design method of anthropomorphic prosthetic hands for reproducing human hand grasping, in: 2014 36th Annual International Conference of the IEEE Engineering in Medicine and Biology Society. IEEE, pp. 6215-6221.

ten Kate, J., Smit, G., Breedveld, P., 2017. 3D-printed upper limb prostheses: a 
review. Disability and Rehabilitation: Assistive Technology, 12(3), 300-314.

Tian, L., Magnenat Thalmann, N., Thalmann, D., Zheng, J., 2017. The Making of a 3D-Printed, Cable-Driven, Single-Model, Lightweight Humanoid Robotic Hand. Frontiers in Robotics and AI, 4, 65.

Van Wyk, K., Culleton, M., Falco, J., Kelly, K., 2018. Comparative Peg-in-Hole Testing of a Force-Based Manipulation Controlled Robotic Hand. IEEE Transactions on Robotics, 34(2), 542-549.

Varol, H.A., Dalley, S.A., Wiste, T.E., Goldfarb, M., 2014. Biomimicry and the Design of Multigrasp Transradial Prostheses, in: Springer Tracts in Advanced Robotics. pp. 431-451.

Vergara, M., Agost, M.J., Gracia-Ibáñez, V., 2018. Dorsal and palmar aspect dimensions of hand anthropometry for designing hand tools and protections. Human Factors and Ergonomics in Manufacturing \& Service Industries, 28(1), 17-28.

Vergara, M., Sancho-Bru, J.L., Gracia-Ibáñez, V., Pérez-González, A., 2014. An introductory study of common grasps used by adults during performance of activities of daily living. Journal of Hand Therapy, 27(3), 225-234.

Wang, S., Hsu, C.J., Trent, L., Ryan, T., Kearns, N.T., Civillico, E.F., Kontson, K.L., 2018. Evaluation of Performance-Based Outcome Measures for the Upper Limb: A Comprehensive Narrative Review. PM\&R, 10(9), 951962.e3.

Weiner, P., Starke, J., Hundhausen, F., Beil, J., Asfour, T., 2018. The KIT Prosthetic Hand: Design and Control, in: 2018 IEEE/RSJ International Conference on Intelligent Robots and Systems (IROS). IEEE, pp. 3328-3334.

Yokokohji, Y., Iida, Y., Yoshikawa, T., 2003. “Toy problem” as the benchmark test for teleoperation systems. Advanced Robotics, 17(3), 253-273. 


\section{Appendix}

To evaluate the grasp type (GT) correctness the following instructions have to be considered for each GT:

- Index pointing, pressing: The GT is considered correct if the palmar side or the tip of distal phalange of the index finger is contacting the object and starting the timer (stopping for maintaining grip score).

- Platform: The GT is considered correct if there is contact between the object and the palm and the angle between any phalange (long fingers and thumb) and the palm is less than $30^{\circ}$.

- Hook: The GT is considered correct if there is contact between the object and the palmar side of at least three long fingers.

- Spherical grip: The GT is considered correct if there is contact between the object and the palmar sides of the thumb, all the phalanges of at least three long fingers and the palm.

- Tripod pinch: The GT is considered correct if the object is contacted by the radial side of the middle finger and by the palmar side of the distal phalanges of the thumb and the index finger.

- Extension grip: The GT is considered correct if there is contact between the object and the palmar side of the distal phalange and the intermediate phalange (if exist) of at least three long fingers and the palmar side of the thumb. In any case, the angle between the distal phalange axes and the object side must be less than $30^{\circ}$. For the boxes the contact of the thumb and finger phalanges must be in the opposing sides of the box with bigger area.

- Cylindrical grip: The GT is considered correct if the angle between the main axis of the thumb and the main axis of the object's grip area is greater than $60^{\circ}$ and there is contact between the object and the palmar sides of the thumb, all the phalanges of at least three long fingers and the palm.

- Diagonal volar grip: The GT is considered correct if the angle between the plane defined by the thumb phalanges and the symmetry plane of the object is less than $30^{\circ}$ and there is contact between the object and the palmar sides of the thumb, the palm and at least three long fingers.

- Lateral pinch: The GT is considered correct if there is contact between the object and, at least, the palmar side of the distal phalange of the thumb and the radial side of the index finger.

- Pulp pinch: The GT is considered correct if the object contacts with the palmar sides of the distal phalange of the thumb and the distal phalange of only one long finger, without any contact of the object with the palm. 


\section{Chapter 3}

Grasping ability and motion synergies in affordable tendon-driven prosthetic hands controlled by able-bodied subjects 

This chapter corresponds exactly to the manuscript submitted to Frontiers in Neurorobotics:

Grasping ability and motion synergies in affordable tendon-driven prosthetic hands controlled by able-bodied subjects

Immaculada Llop-Harillo, Antonio Pérez-González, and F. Javier

Andrés

\begin{abstract}
Affordable 3D-printed tendon-driven prosthetic hands are a rising trend because of their availability and easy customization. Nevertheless, it exists a lack of comparative studies about the functionality of this kind of prostheses. The tradeoff between the number of actuators and the grasping ability of the prosthetic hands is a relevant issue for their design. The analysis of synergies among fingers is a common method used to reduce dimensionality without losing dexterity significantly. Therefore, the purpose of this study is the assessment of functionality and motion synergies of different tendon-driven hands using an able-bodied adaptor. The use of this adaptor to control the hands using the fingers of healthy subjects allows taking advantage of the human brain control while obtaining the synergies directly from the artificial hand. Four artificial hands (IMMA, Limbitless, Dextrus v2.0, InMoov) were confronted with the Anthropomorphic Hand Assessment Protocol, quantifying functionality and human-like grasping. Three subjects performed the tests by means of an especially designed able-bodied adaptor that allows controlling each tendon by a different human finger. The tendon motions were registered and correlation and principal component analyses were used to obtain the motion synergies. The grasping ability of the analyzed hands ranged between $48 \%$ and $57 \%$ with respect to that of the human hand, obtaining the IMMA hand the highest score. The subject's effect was found non-significant on the grasping ability score. For all the hands, the highest tendon pair synergies were obtained for pairs of long fingers, being greater for adjacent fingers. The principal component analysis showed that, for all the hands, two principal components explained near or more than $80 \%$ of the variance. Several factors such as the friction coefficient in the hand contact surfaces, limitations on the underactuation or for a correct thumb opposition, need to be improved in this type of prostheses to increase its grasping stability. The principal components obtained in this study provide useful information for the design of transmission or control systems to underactuate these hands.
\end{abstract}




\section{Keywords}

3D printing, anthropomorphic hand, experimental analysis, functional testing, grasping, prosthetic hand, synergies

\section{Introduction}

The advent of 3D-printing technology in the prosthetics or orthotics industries has led to the generation of affordable and customized designs. These designs attempt to meet the most basic needs in the shortest time and with the least money (typically less than $\$ 500$ for a hand prosthesis (ten Kate et al., 2017)). For the purpose of this research, being affordable refers primarily to prosthetic hands printed using fused deposition modeling (FDM) technology, which is becoming popular under the premise of do-ityourself (DIY) ("RepRap," 2017). Lots of these hand designs can be freely downloaded from Computer-Aided Design (CAD) repositories such as www.instructables.com and www.thingiverse.com, or from government institutions such as the NIH 3D print exchange ("U.S. Department of Health and Human Services - National Institutes of Health,” 2017). Non-profit initiatives such as Open Hand Project (Gibbard, 2013), e-NABLE ("eNABLE,” 2014), or Openbionics (Gibbard, 2018) are the main source for such repositories. Anyone can download a CAD model, typically a stereolithography file (.stl), and print it. Most designs require additional elements for final assembly, such as screws/bolts, elastic cords, nylon cords, and Velcro ${ }^{\circledR}$, which should be readily available (Burn et al., 2016). Evidently, there is some debate about the fact that these designs may not be recognized as medical devices since their manufacturers are not subject to controls by national Regulatory Authorities (Asanuma, 2012a, 2012b). Nevertheless, this undeniable trend of accessible production has motivated some revisions in recent years (Burn et al., 2016; Phillips et al., 2015; Tanaka and Lightdale-Miric, 2016; ten Kate et al., 2017) and continues to encourage deeper analysis. Owing to the lack of comparative studies on the functionality of this kind of prostheses, addressing this gap in the literature is one of the aims of this paper.

Controzzi et al. (Controzzi et al., 2014) specified six important issues to consider during the design and development phases of a prosthetic hand. Having a deeper insight into the affordable designs but taking these issues into account can help to establish links between the design process and the usage environment: 
(a) Kinematic architecture: refers to the mechanical concept in which degrees of freedom (DoFs) are related to the required degrees of actuation (DoAs), independently of questions (b) and (c). Underactuated mechanisms are those with fewer DoAs than DoFs, usually linking the movement among the joints of each finger.

(b) Actuation principle: all early affordable designs were body-powered. While the body-powered devices may perform multiple tasks, their fingers bend together and users have difficulty grasping the object as tightly as possible (Dally et al., 2015). The DIY has not eluded using the latest open-source developments to get electric prostheses, like the Arduino microcontrollers (www.arduino.cc) together with compact DC motors and compact batteries.

(c) Actuation transmission connects (a) with (b): the power transmission from the actuators to the fingers should be stiff during the flexion, but avoiding any damage due to haphazard impacts on the dorsum. The spontaneous adaptation to the shape of the grasped object is also desirable in terms of stability. These two reasons and the easiness of assembly make the use of nylon threads running through sheaths the most common transmission in low-cost designs.

(d) Sensors: for the scope of affordable devices, both assembly and maintenance are far easier when the hand is used as an open-loop device.

(e) Materials, and (f) Manufacturing method, are already strongly related with the FDM technology considered: acrylonitrile butadiene styrene (ABS) and the polylactic acid (PLA) are the same thermoplastic materials used in conventional orthotics, with similar biocompatibility, stability, durability, and mechanical properties (Burn et al., 2016; Ventola, 2014). Moreover, the use of compliant materials such as Ninjaflex ${ }^{\circledR}$ in the manufacturing of joints may avoid using an additional extension system for the fingers.

A compromise between the number of motors and the grasping ability of the prosthetic hands appears during the design process. Fewer motors allow reducing weight and cost, but at the expense of lesser motion versatility. Although underactuation, from issue (a), may suppose a loss of dexterity, it is the preferred architecture in a DIY context due to its ease of assembly and maintenance. As compensation and from the perspective of issue (b), the use of electric prostheses enhances the dexterity by controlling each finger independently. Moreover, the analogy of nylon threads acting like the tendons in the human hand, issue (c), also allows the actuators to be located 
remotely, in the palm or forearm space, reducing the dimensions and weight of the fingers.

In this study, the hands selected meet the common characteristics described above, with no-feedback from sensors, tendon transmissions as the preferred option, and being done with regular FDM materials. It will be denoted how their designers resolved the issues previously enumerated, also from our own experience in the assembly process.

The analysis of the motion synergies or eigenpostures among fingers for a hand with more DoFs than DoAs could be a method to aid in the decision making at the early steps of the design. In the literature (Santello et al., 2016), synergies have successfully applied to create novel design and control concepts for artificial hands. Roboticists have proposed synergies applicable to the pre-grasping phase to reduce the number of DoFs that can be controlled in an independent manner. The fingers of the affordable hands for which the motions of the tendons are highly correlated can be candidates to be moved by a single controller, or even a single actuator. The recent reviews of Salvietti (Salvietti, 2018) and Santello et al. (Santello et al., 2016) prove that the approach of replicating human hand synergies onto robotic hands has been the aim of some studies in the last decade. The review of Salvietti (Salvietti, 2018) shows the main solutions in the literature for the reduction of dimensionality by coupling some DoFs of the robotic hands, both at software and hardware levels. Most of them were based on the human hand postural synergies defined in Santello et al. (Santello et al., 1998). According to the software synergies, two main categories were highlighted by Salvietti: (i) mapping of synergies from humans to robots using the data collected from the human hand, and (ii) defining the synergies for robotic hands collecting data from grasps obtained directly with the robotic hand. From this study, the first category may allow benefiting from the highly evolved control model that the human brain is, but it has the difficulty of adapting the data to the kinematics of a robotic hand. The second category allows obtaining very specialized synergies for the specific hand kinematics, but may highly depend on the set of grasps selected. However, to our knowledge, no previous work, with the exception of a preliminary study by the authors (Llop-Harillo and Pérez-González, 2017a) on a hand prototype, have analyzed the motion synergies of tendon-driven prosthetic hands (TDPHs) with each DoA being controlled by a finger of a healthy human subject, that is, taking the advantage of the human brain control mechanisms while obtaining the data directly from the artificial hand. Such data would summarize the positive characteristics remarked by Salvietti (Salvietti, 
2018), therefore being specialized for the specific device kinematics. This approach is used in the present study and a comparison of the synergies obtained for different TDPHs will be analyzed in the paper.

In the research literature, most of the upper limb prostheses are still evaluated through subjective protocols or questionnaires on end-users (Lindner et al., 2010). However, the use of an able-bodied adaptor (ABA) to adapt the prosthesis to a healthy subject, has proven its usefulness in preliminary functional assessments (Bouwsema et al., 2014; Dalley et al., 2012; Fougner et al., 2014; Huinink et al., 2016; Kyberd, 2011; Rossi et al., 2017; Smit et al., 2015; Vasluian et al., 2014) prior to any test involving more sensitive potential users. The majority of these functional assessments used objective protocols as the Southampton Hand Assessment Procedure (SHAP) (Light et al., 2002), the Box and Block Test (BBT) (V. Mathiowetz et al., 1985) or the Nine Hole Peg Test (NHPT) (Virgil Mathiowetz et al., 1985). Notwithstanding, all these tests are designed and commonly used to assess the human hand function. Moreover, BBT and NHPT use a limited variety of grasping types and objects. In a recent study by the authors (LlopHarillo et al., 2019) a benchmark to assess the grasping ability of anthropomorphic artificial hands was presented. This benchmark (LlopHarillo et al., 2019) is composed of 26 tasks involving grasping with the eight most relevant human grasp types (GTs) during activities of daily living (ADLs) and two non-grasping postures. The set of grasps selected in this benchmark accounts for more than $90 \%$ in grasp frequency according to several previous studies found in the literature (Bullock et al., 2013; Feix et al., 2016; Light et al., 2002; Sollerman and Ejeskär, 1995; Vergara et al., 2014; Wang et al., 2018).

In this study we face some questions resulting from the approaches presented above: Are the 3D-printed publicly available prostheses functional to perform ADLs? Are there significant differences in functionality among existing models or among the GT intended? Which are the main limitations from a mechanical point of view for functional grasping with these prostheses? Is the subject effect significant on the grasping ability of the hands when using an ABA? Which are the motion synergies on TDPHs with a human control strategy?

The objective of this paper is to evaluate and compare the functionality and human-like grasping of several 3D-printed affordable TDPHs using a publicly available experimental protocol published by the authors (LlopHarillo et al., 2019). The main difficulties of these artificial hands for 


\section{0 | Chapter 3}

performing the most common grasps in ADLs are analyzed. A custom-made ABA is used in order to be able to control each of the hand tendons independently with the motion of one healthy subject finger. In this way, the control of the hand is performed by the subject and the specific solutions taken by the designers for controlling or underactuating the hand are excluded from the comparison. Thus, the hands are compared in terms of their kinematic chain, finger segments geometry and the characteristics of the contact surfaces: materials, stiffness, friction coefficients. Additionally, the ABA includes suitable electronics to measure and register the displacement of the tendons during the grasping process enabling the analysis of the motion synergies employed. It is also an objective of this paper to analyze the effect of the subject on the control of these TDPHs using the ABA.

\section{Materials and methods}

\subsection{Tendon-driven prosthetic hands}

Three of the most common 3D-printed electrical prosthetic hands for transradial amputees currently available online, with documented clinical usage, were chosen for the present study, namely (Andrés et al., 2019): the Dextrus v2.0, the InMoov, and the Limbitless hands. Together with the IMMA hand, designed by the authors (Llop-Harillo and Pérez-González, 2017a), Table 1 summarizes each of the mechanical design characteristics noted by Controzzi et al. (Controzzi et al., 2014) as described in the previous section. Note that the actuation transmission was made with nylon threads for all of them, none has any sensors, and the manufacturing method was FDM. As they try to mimic human hand anatomy, the joints of the fingers and thumb are named by analogy, from distal to proximal: distal interphalangeal (DIP), proximal interphalangeal (PIP), metacarpophalangeal (MCP), and carpometacarpal (CMC). Details regarding the actuation principles are those obtained from the download source together with the CAD model. All hands were printed using a Colido ${ }^{\circledR}$ mod. X3045 printer with Repetier-Host software. Prior to that, a wrist supplement was added by means of SolidWorks CAD software to ease the fixation to the ABA designed. Time and cost have been estimated on the basis of the model of printer and materials employed. Note that the same $0.8 \mathrm{~mm}$ nylon fishing line (ultimate tensile strength of $220.5 \mathrm{~N}$ ) was employed as a tendon for each finger of every hand. It pierces all phalanges up to the distal end of the finger, 
where it is to be knotted. Further details are provided in Table 1 and hereunder.

Table 1. Main design characteristics of the selected 3D-printed hand prostheses.

\begin{tabular}{|c|c|c|c|c|}
\hline $\begin{array}{l}\text { Hand model } \\
\text { (source) }\end{array}$ & $\begin{array}{l}\text { IMMA (Llop- } \\
\text { Harillo and } \\
\text { Pérez- } \\
\text { González, } \\
\text { 2017b) }\end{array}$ & $\begin{array}{l}\text { Limbitless } \\
\text { (UCFArmory and } \\
\text { Enablingthefuture, } \\
\text { 2014) }\end{array}$ & $\begin{array}{l}\text { Dextrus v2.0 } \\
\text { (Gibbard, } \\
\text { 2015) }\end{array}$ & $\begin{array}{l}\text { InMoov } \\
\text { (Langevin, } \\
\text { 2013) }\end{array}$ \\
\hline & \pm & & & \\
\hline $\begin{array}{c}\text { Kinematic } \\
\text { architecture* }\end{array}$ & $\begin{array}{c}\text { Underactuated } \\
\text { (15 DoF }>6 \\
\text { DoA })\end{array}$ & $\begin{array}{c}\text { Underactuated } \\
\text { (14 DoF > } 1 \text { DoA) }\end{array}$ & $\begin{array}{l}\text { Underactuated } \\
\text { (15 DoF > } 5 \\
\text { DoA) }\end{array}$ & $\begin{array}{l}\text { Underactuated } \\
\text { (17 DoF > } 5 \\
\text { DoA })\end{array}$ \\
\hline $\begin{array}{l}\text { Number of } \\
\text { joints }\end{array}$ & $\begin{array}{c}\text { 3f [DIP, PIP, } \\
\text { MCP }] \\
\text { 3th [DIP, MCP, } \\
\text { CMC }]\end{array}$ & $\begin{array}{c}3 f \text { [DIP, PIP, MCP] } \\
\text { 2th [DIP, MCP] }\end{array}$ & $\begin{array}{c}\text { 3f [DIP, PIP, } \\
\text { MCP] } \\
\text { 3th [DIP, MCP, } \\
\text { CMC] }\end{array}$ & $\begin{array}{c}\text { 3f [DIP, PIP, } \\
\text { MCP] }+1 \text { [CMC } \\
\text { at ring and little } \\
\text { fingers] } \\
\text { 3th [DIP, MCP, } \\
\text { CMC] }\end{array}$ \\
\hline $\begin{array}{l}\text { Actuation } \\
\text { principle }\end{array}$ & Not defined & $\begin{array}{c}1 \text { Servo motor } \\
\text { (Hitec HS-5645MG } \\
\text { - Digital High } \\
\text { Torque MG Servo) }\end{array}$ & $\begin{array}{c}5 \text { DC linear } \\
\text { actuators } \\
\text { (Actuonix } \\
\text { PQ12-63:1 } \\
\text { linear actuator) }\end{array}$ & $\begin{array}{c}5 \text { Servo motors } \\
\text { (either } \\
\text { HobbyKing } \\
\text { HK15298, } \\
\text { Tower Pro } \\
\text { MG995, or } \\
\text { equivalent) }\end{array}$ \\
\hline $\begin{array}{c}\text { Motors } \\
\text { location** }\end{array}$ & - & FArm & Palm & FArm \\
\hline $\begin{array}{l}\text { Materials } \\
\text { (infill) }\end{array}$ & $\begin{array}{c}\text { PLA-Soft }{ }^{\circledR}(20- \\
40 \%) / \\
\text { Filaflex }^{\circledR} \\
\text { (pads) }(20- \\
30 \%) / \\
\text { NinjaFlex }^{\circledR} \\
\text { (joints) }(40 \%)\end{array}$ & $\begin{array}{c}\text { PLA (25\%) / } \\
\text { Ninjaflex }{ }^{\circledR} \text { (joints) } \\
(25 \%)\end{array}$ & $\begin{array}{l}\text { Ninjaflex }^{\circledR} \\
(35 \%)\end{array}$ & PLA (30\%) \\
\hline Total weight (g) & 131.5 & 144.5 & 131 & 201.5 \\
\hline
\end{tabular}




\section{2 | Chapter 3}

\begin{tabular}{ccccc}
$\begin{array}{c}\text { Cost 3D-printing } \\
\text { materials } \\
\text { Printing time }\end{array}$ & $\$ 5 \mathrm{~h}$ & $16 \mathrm{~h}$ & $\$ 11$ & $\$ 6$ \\
\hline $\begin{array}{c}\text { Dimensions*** } \\
{[\mathrm{HL} / \mathrm{HB}](\mathrm{mm})}\end{array}$ & $184 / 80$ & $200 / 89$ & $185 / 87$ & $22 \mathrm{~h}$ \\
\hline Clinical usage & - & $\begin{array}{c}\text { (“Limbitless } \\
\text { solutions,” 2015; } \\
\text { Owen, 2018) }\end{array}$ & (Alec, 2015) & (Huchet, 2014) \\
\hline
\end{tabular}

(*) 3f: three joints at fingers; 3th: three joints at the thumb; 2th: two joints at the thumb. DoA as originally intended for each model.

(**) Palm: inside palm (or on palm dorsum for control board and battery); FArm: Actuators/control board/battery in forearm.

$(* * *)$ HL: hand length (from the most proximal palmar point to the tip of the middle finger); HB: hand width (at the metacarpal heads)

\subsubsection{IMMA Hand}

The IMMA hand is a tendon-driven anthropomorphic prosthetic hand prototype with six DoAs, designed at the Universitat Jaume I by the authors (Llop-Harillo and Pérez-González, 2017a). Flexion and circumduction movements of the thumb are actuated separately with two different nylon threads, allowing opposition of the thumb to orient its distal phalanx to the distal phalanges of the fingers. Therefore, it contains a total of six tendons: five for the flexion of the fingers and one additional for the circumduction of the thumb. For the sake of simplicity in the assembly, and similarly to the Limbitless Hand, it uses elastic elements at the joints to drive extension movements when the tendons are released. Its main dimensions are between the 50th percentiles of the male and female human hands, based on data obtained in the authors' research group (Vergara et al., 2018).

It was 3D-printed by FDM combining PLA SOFT-Flexible ${ }^{\circledR}$ : mixture of PLA and thermoplastic polyurethane (TPU) using hexamethylene diisocyanate, for the palm and phalanges; NinjaFlex ${ }^{\circledR}$ : special formulation of TPU with high flexibility and durability, for the elastic joints; and FilaFlex ${ }^{\circledR}$ : based on TPU with additives, for the finger and palm pads. The pads located in the main contact areas with objects aim to mimic the friction coefficient of the human hand skin. The IMMA hand is licensed under a Creative Commons (CC BY-NC-ND 4.0: Attribution-NonCommercialNoDerivatives) license. 


\subsubsection{Hand of the Limbitless arm}

Developed by the University of Central Florida Armory, the Limbitless Arm ("Limbitless solutions," 2015) is the first myoelectric design available from the e-NABLE site ("e-NABLE," 2014). It is based on the bodypowered Flexy-Hand (Steve Wood, 2014). It is available in two versions in the same download (UCFArmory and Enablingthefuture, 2014): with no palmar abduction of the thumb, and with the thumb with a palmar abduction of $45^{\circ}$. This last was the model used in the present research. It has five tendons, one for the flexion of each finger, and it is originally intended to work with one actuator, thus closing fingers and thumb altogether. Instead, for the scope of this research, this issue is surpassed to operate each finger independently, as done in the other models. The Limbitless arm is licensed under a Creative Commons (CC BY-NC 3.0: Attribution-NonCommercial) license.

\subsubsection{Dextrus v2.0}

The Dextrus hand, in its second version available at (Gibbard, 2015), showed significant changes with regard to the first version. It is completely made of flexible material (Ninjaflex ${ }^{\circledR}$ ) and so it makes flexible joints to be fully integrated within the design. It has five tendons, one for the flexion of each finger, and uses five linear actuators embedded in the palm. This initial advantage could difficult resizing for smaller hands, for example for children. Nevertheless, it was printed in its default size. For this research, the linear actuators are not included and the tendons are extended to the forearm for actuation with the ABA described below.

Its rubberized unibody design makes this hand very easy to assemble: the tendons only need to be routed to make the fingers mechanically compliant when being forced closed. It makes replacing individual fingers impossible if broken. Dextrus v2.0 is licensed under a Creative Commons (CC BY-SA 4.0: Attribution-ShareAlike) license.

\subsubsection{InMoov}

The InMoov prosthetic hand (Langevin, 2013) was originally launched in 2012, as a part of an Open Source 3D printed life-size robot. Each finger can be mounted in order to achieve an active two-way control, flexion and extension. For the sake of simplicity and to evaluate grasping capabilities, an elastic band was used here for finger extension. This hand has five tendons for the flexion of the fingers but with the singularity of additional joints in 


\section{4 | Chapter 3}

the little and ring fingers near to the center of the palm to reproduce the palmar arch. Cut bike spokes were used for thumb and finger joints, and a bolt for the palmar joint. The InMoov hand is licensed under a CC BY-NC 3.0 license.

\subsection{Experiments}

For the scope of this research, each affordable 3D-printed prosthesis was confronted with the Anthropomorphic Hand Assessment Protocol (AHAP) (Llop-Harillo et al., 2019), described briefly below. Three able-bodied subjects assessed the performance of the four hands by means of an ABA, described in a subsequent section. All subjects were right-handed and they adapted the prostheses to their right arm. All subjects had a similar user experience with the ABA and the artificial hands. Once removed the electrical actuation of the hands, the ABA allows the user to move each DoA of the artificial hand by pulling with their own fingers the individual cords (tendons) with a ring attached at the end of each one. This actuation method allows splitting any control and actuation issue from the evaluation of the mechanical performance of the artificial hand and transfers the control of the artificial hand to the human brain. To avoid fatigue effects, each subject tested each prosthesis in a different session on different days. Each of the twelve experiments ( 3 subjects and 4 prostheses) was video-recorded and lasted approximately 60-110 min. After attaching the prosthesis to the subject's forearm using the ABA, the subject was given some time to get familiarized with the hand actuation and then proceeded with the AHAP following the experimenter instructions. The Ethics Committee of the Universitat Jaume I (UJI) approved the study and written informed consent was obtained from all the participants. The three adult male subjects who participated in the experiment were free from hand pathologies or injuries (see characteristics in Table 2, the grip strength of their right hand was measured with a CAMRY ${ }^{\circledR}$ Digital Hand Dynamometer).

Table 2. Characteristics of the study sample.

\begin{tabular}{ccccc}
\hline Subject & Age & $\begin{array}{c}\text { Hand length } \\
(\mathrm{mm})\end{array}$ & $\begin{array}{c}\text { Hand width } \\
(\mathrm{mm})\end{array}$ & $\begin{array}{c}\text { Grip strength } \\
(\mathrm{kgf})\end{array}$ \\
\hline S1 & 53 & 190 & 91 & 44.2 \\
S2 & 24 & 194 & 94 & 39.3 \\
S3 & 39 & 181 & 86 & 48.7 \\
\hline
\end{tabular}




\subsubsection{Anthropomorphic Hand Assessment Protocol (AHAP)}

The AHAP (Llop-Harillo et al., 2019) is a validated benchmark to quantify the grasping ability of anthropomorphic hands. It is composed of 26 tasks (Figure 1) involving the eight most common human GTs in ADLs: pulp pinch (PP), lateral pinch (LP), diagonal volar grip (DVG), cylindrical grip (CG), extension grip (EG), tripod pinch (TP), spherical grip (SG), and hook $(\mathrm{H})$; and two non-grasping postures: platform (P), and index pointing/pressing (IP). Three objects per GT and one for each non-grasping posture are used. These objects were selected with different size, shape, weight, texture, and rigidity, from the publicly available Yale-CMUBerkeley Object and Model Set (Calli et al., 2015). For each task the object is handed over to the subject by an experimenter holding it in the correct position for the successful execution of the grasp and the experimenter releases it once the grasp has been performed by the artificial hand. Prior to the trials to be evaluated, the experimenter indicates the correct grasping posture for each object/task and the subject should try to reproduce it as accurately as possible after one minute of pre-practice. In the test, the subject has to actuate the artificial hand for grasping the object with the palm pointing upwards and maintain the grasp for three seconds. If the grasp is completed with the correct GT the score for that trial is 1 point, if the grasping posture is different from the one specified the score is 0.5 points, and if the artificial hand cannot grasp the object the score is 0 . In the first two cases and with the object still grasped, the next step of the protocol consists of rotating the wrist of the subject $180^{\circ}$ for the palm pointing downwards and holding the object for three additional seconds in this pronated position. For this step if there is no visible motion of the object with respect to the hand the score is 1 point, if the object moves with respect to the hand but is not dropped the score is 0.5 , and if the object is dropped 0 points. These steps must be repeated 3 times for each object. This benchmark allows quantifying the functionality and human-like grasping of artificial hands through a numerical total Grasping Ability Score (GAS). Its reliability, consistency and responsiveness have been statistically validated (LlopHarillo et al., 2019). To obtain the GAS for each hand, the AHAP considers both grasp correctness (human-like) and stability after rotating the artificial hand from supination to pronation. For a better analysis, the GAS score can be split into the two terms corresponding to the grasp correctness (grasping) and the stability under motion (maintaining). A partial GAS, for each of the ten GTs/postures, can also be obtained. For the GTs, the scores for the three objects are added to obtain the partial GAS for each GT. As the GAS is 


\section{6 | Chapter 3}

normalized, it can be expressed as a percentage of human grasping ability where $100 \%$ corresponds to the healthy human hand.

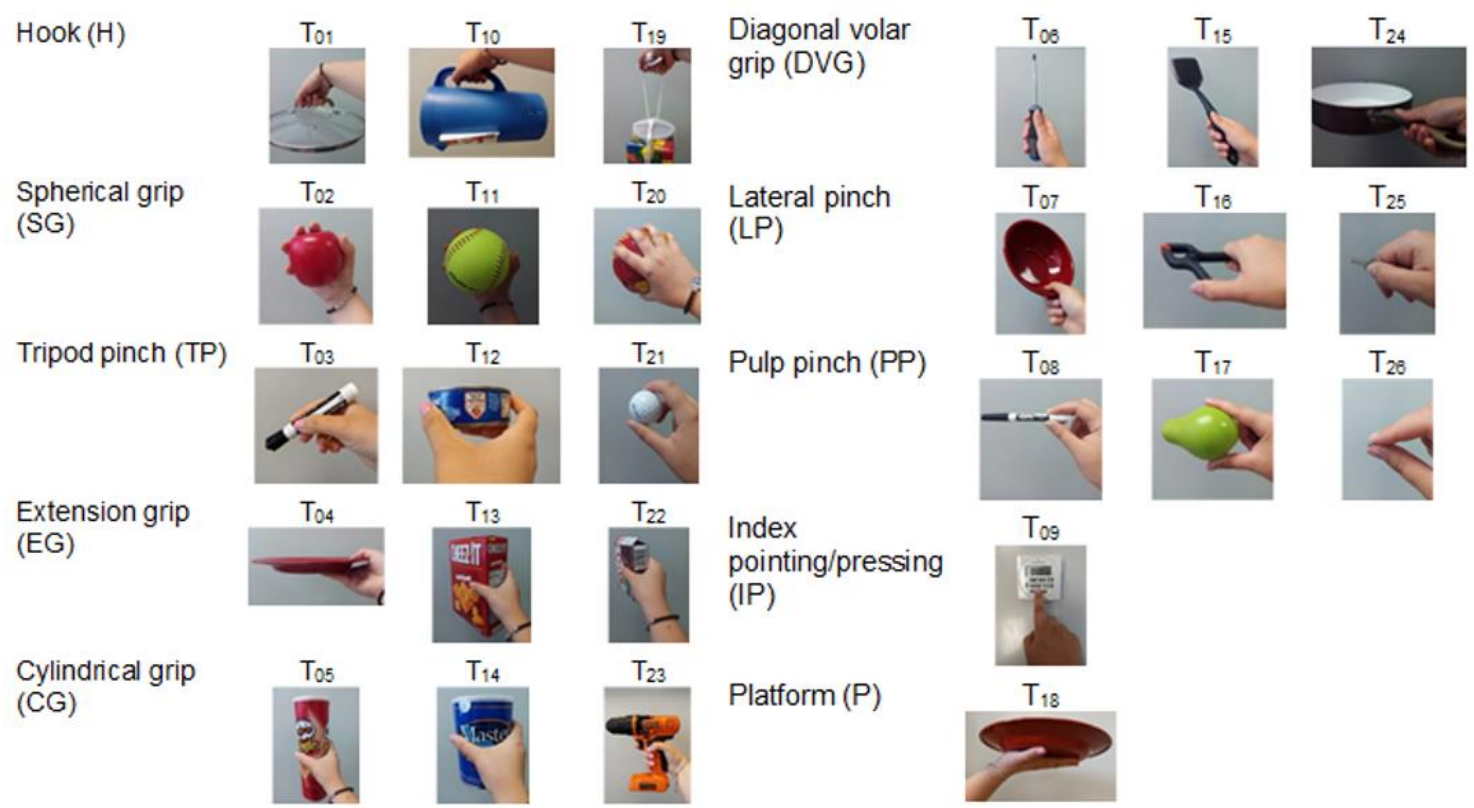

Figure 1. Tasks $\left(\mathrm{T}_{\mathrm{i}} ; \mathrm{i}\right.$ : indicates the task order in the protocol) of the Anthropomorphic Hand Assessment Protocol (AHAP) (Llop-Harillo et al., 2019)

\subsubsection{Able-bodied adaptor ( $A B A)$}

An adaptor specifically designed is needed to perform the AHAP with TDPHs controlled by healthy human subjects. In a previous preliminary study by the authors (Llop-Harillo and Pérez-González, 2017a), a first design of an ABA was developed to measure the cable excursion of TDPHs when they are controlled by a human subject. However, one of the problems of this first ABA was that the final position of the artificial hand was about $35 \mathrm{~cm}$ more distal than the sound hand of the user forcing to unnatural compensatory movements of the arm. In the present study that ABA was redesigned, leading to a lower distal separation of the artificial hand with respect to the own user's hand (Figure 2). In addition, the friction introduced in the tendon motions is lower than in the previous version. The ABA is connected to the forearm of an able-bodied subject and allows to control with their fingers any TDPH moved by up to six tendons. It is composed of a TRS prosthetics' Pro Cuff ${ }^{\circledR}$ and an own designed PLA 3D-printed structure. It registers the tendon excursions during the motion of the hand through six linear potentiometers BOURNS $^{\circledR}$ PTB6043-2010BPB103, with a measurement range of $60 \mathrm{~mm}$, connected to an Arduino ${ }^{\circledR}$ Mega 2560 board. The system also includes an HC-05 Bluetooth module, which enables 
wireless communication. The electronics is powered by a 9V battery, which is connected to the power jack of the Arduino board.

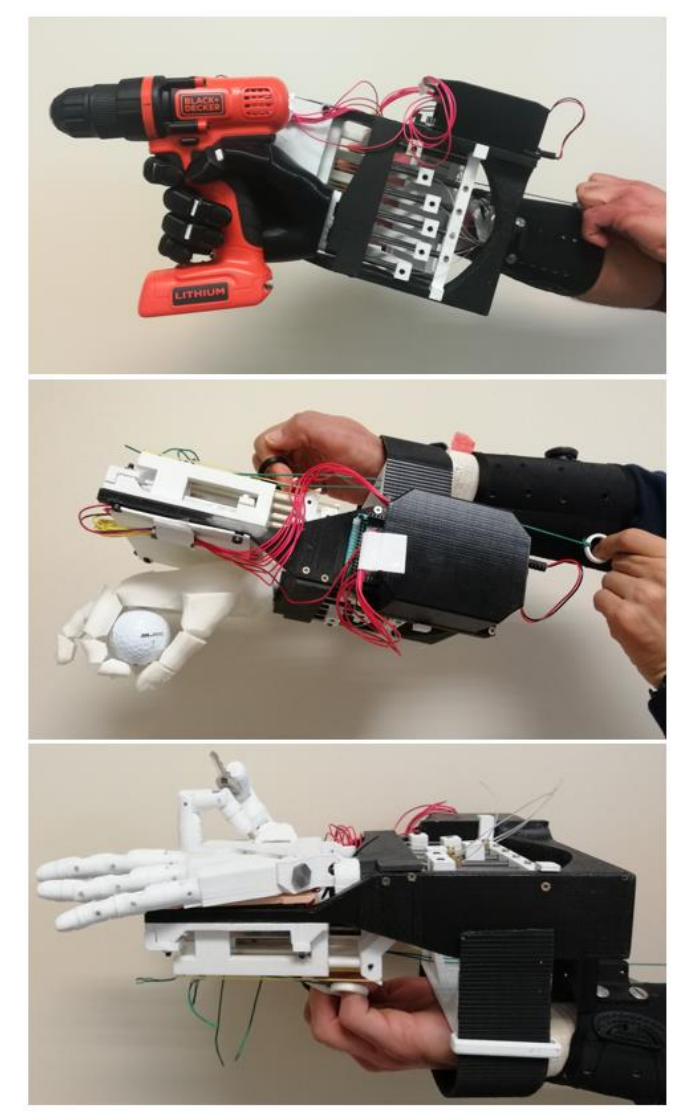

Figure 2. Different views of the able-bodied adaptor (ABA).

\subsubsection{Acquisition of grasping data}

The data acquisition software developed permits to register the displacement of the six linear potentiometers, measuring the displacement of the different tendons that actuate the artificial hand during the tests performed. For this purpose, the Arduino board reads the values of the six potentiometers using six analog inputs and sends these values by Bluetooth communication to a laptop computer. Using Matlab ${ }^{\circledR}$, these measures are stored in a matrix along with the time. The data recording process is divided into tasks and repetitions.

\subsection{Data analysis}

Firstly, the GAS obtained with the different artificial hands was analyzed and compared. For a better understanding of the results, the GAS was also analyzed splitting the score into the two parts corresponding to grasping and maintaining steps of the protocol (see section describing the AHAP above). 


\section{8 | Chapter 3}

The partial GAS corresponding to each GT/posture was also compared for the different hand prostheses.

Secondly, a two-way analysis of variance (ANOVA) on total GAS with factors 'subject' and 'hand' and a three-way ANOVA on partial GAS with factors 'subject', 'hand' and 'GT/posture' were conducted to ascertain the effect of the different factors involved in the total or partial GAS. A post-hoc analysis (HSD Tukey) was performed for a deeper analysis of the significant factors.

Thirdly, a group of analyses using Pearson Correlation Coefficient (CC) and Principal Component Analysis (PCA) among tendon displacements was undertaken to study the motion synergies among fingers in the different hands. For the analysis of the correlation between tendon displacements in a grasping task repetition, the instantaneous displacements recorded from the start of the task to the establishment of the grasp were stored in a data matrix with columns corresponding to the different potentiometers (tendons). To avoid unwanted effects derived from noise in the potentiometers signal, displacements lower than $0.2 \mathrm{~mm}$ were rounded to zero. The grasp was considered established once all the tendons reach their maximal displacement, excluding inactive tendons during the grasp. For the subsequent analyses, only the data of the grasps performed successfully (those with score 1 or 0.5 in the grasping step of the AHAP) were considered. For each successful repetition of each task, the CC among any pair of columns of the data matrix was computed. The correlation between a pair of tendons was considered high in a grasping task repetition if the CC of the corresponding columns is higher than 0.9. For each pair of tendons, the percentage of successful grasps for which the CC was greater than 0.9 is a measure of the tendon pair synergy (TPS). A TPS near to 1 indicates that this pair of tendons could be controlled with the same actuator, while a TPS near to 0 indicates a null correlation for this tendon pair. TPSs were compared for the different hands and subjects. A two-way ANOVA on TPS with factors 'subject' and 'tendon pair' was conducted for each hand. Moreover, the data matrices of tendon displacements obtained following the procedure above described and corresponding to all tasks and repetitions with successful grasping where stacked in a single matrix for each hand and subject. A PCA was conducted on these stacked data matrices of tendon displacements. The PCA could allow reducing the dimensionality corresponding to the tendon displacements in order to design underactuation systems for simpler control of the hands. The PCA was performed in two different ways: separately for each subject and including all the data for the three subjects together. 
Matlab $^{\circledR}$ was used to obtain the data matrices to compute TPSs and the built-in function corrcoef to compute the CC. SPSS statistical package (version 25, SPSS Inc, Chicago, USA) was used for the statistical analyses. The PCA extraction method was based on a fixed number of factors to explain more than $80 \%$ of the variance and the rotated component matrices were obtained using the Varimax with Kaiser Normalization as rotation method.

\section{Results}

\subsection{Grasping Ability Score (GAS)}

Table 3 shows the mean GAS and its standard deviation across subjects, obtained with the AHAP for each prosthetic hand analyzed. Moreover, it includes the detail of the score corresponding to the two parts of the protocol, grasping and maintaining.

Table 3. Grasping Ability Score (GAS $\pm S D$ ) obtained with the AHAP.

\begin{tabular}{cccc}
\hline Hand & Grasping (\%) & Maintaining (\%) & GAS (\%) \\
\hline IMMA & $77 \pm 1$ & $37 \pm 4$ & $57 \pm 2$ \\
Limbitless & $63 \pm 2$ & $37 \pm 4$ & $50 \pm 3$ \\
Dextrus & $61 \pm 3$ & $34 \pm 6$ & $48 \pm 4$ \\
InMoov & $57 \pm 1$ & $40 \pm 2$ & $49 \pm 1$ \\
\hline
\end{tabular}

These results state that the four hands are below $60 \%$ in terms of grasping ability with respect to the human hand (100\%). It is relevant that in the grasping part of the AHAP the scores are over $55 \%$ in all hands whereas, in the maintaining part, they are below $40 \%$. This result shows that holding the object securely under motion of the hand is more demanding than just configuring the hand for grasping in a human-like manner. IMMA is the hand performing better for grasping (77\%) and scores the highest GAS (57\%). Dextrus v2.0 is the hand performing worse the part of the score corresponding to the maintaining step (34\%) and scores the lowest GAS $(48 \%)$.

Figure 3 shows the partial GAS of the analyzed TDPHs for each common GT/posture in ADLs. It can be noticed that the P posture was perfectly performed (100\%) by all hands except the Limbitless, due to the orientation 


\section{0 | Chapter 3}

and position of its thumb. The IP posture, needed for example to type with a keyboard, is the one that has obtained the best results on average for the four hands analyzed, followed by $\mathrm{H}$ and TP, with scores over 70\%. However, the partial GAS for EG and PP are below 30\%. The low results for EG can be explained by the difficulty, according to the mechanical design of TDPHs, in keeping extended the distal segments of the fingers while flexing their MCP joints. In the case of the PP, the issues come up due to the limitations for the correct opposition between the thumb and the index finger, reliant on the orientation of the rotation axes of the thumb. The differences shown among the analyzed TDPHs in the LP are also attributed to the design of the thumb opposition, but in this case regarding the radial side instead of the palmar side of the index finger. The additional DoA of the thumb for the IMMA hand is reflected in a better performance for PP, TP and especially for LP. This problem of the orientation and mobility limitations of the thumb, together with the limitations imposed on the palm opposition by almost flat palm designs, also led to a discrete performance (around 50\% in partial GAS) in the DVG. Two additional problems appear in power grasps such as DVG, EG, SG and CG, especially with bigger or heavier objects: the limited grasping force that can be exerted due to the losses in mechanical advantage, and the low compliance and friction coefficient that is obtained in the analyzed TDPHs in the hand-object contact areas.

The ANOVA tests showed that the subject's effect was non-significant on the GAS $(p=.234)$ nor on the partial GAS $(p=.786)$. By contrast, the hand's effect was found significant on both the GAS $(\mathrm{p}=.010)$ and the partial GAS $(p=.037)$. The post-hoc analysis showed that the IMMA hand performed significantly better than the other three hands and that the differences among the other three hands were non-significant. In addition, the results on partial GAS were significantly different $(\mathrm{p}<.001)$ depending on the GT/posture. An analysis by GT/posture showed that the partial GAS changed significantly with the hand for CG ( $\mathrm{p}=.002)$, DVG ( $\mathrm{p}=.027)$ and LP $(p=.009)$. It is relevant to note that, for these three GTs, the partial GAS had intermediate values, namely: mean values of 38\% for CG, 50\% for DVG and $63 \%$ for LP. The partial GAS for the P posture was also significantly different for the Limbitless (0\%) as compared to the other hands (100\%). For CG, the InMoov obtained a significantly worse result; for DVG, the Limbitless obtained a better result than the other hands and the IMMA the worst one; for LP, the IMMA obtained a better result and the Dextrus v2.0 the worst one. In none of the GT/postures the subject had a significant effect on the partial GAS. 


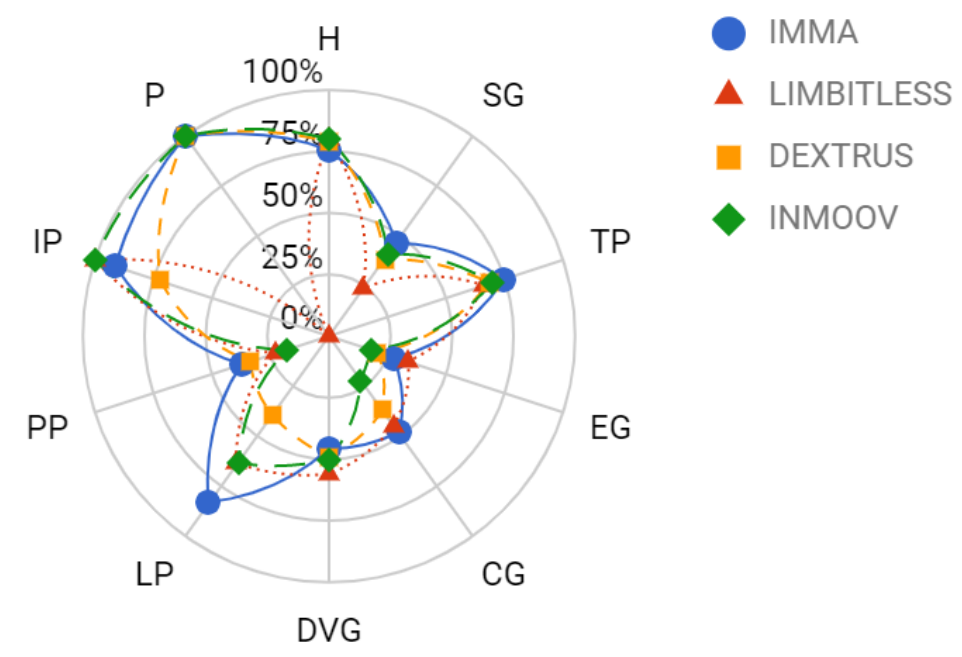

Figure 3. Mean partial GAS grouped by GT (PP: pulp pinch, LP: lateral pinch, DVG: diagonal volar grip, CG: cylindrical grip, EG: extension grip, TP: tripod pinch, SG: spherical grip, H: hook, P: platform, IP: index pointing/pressing) obtained by each prosthesis through the AHAP.

\subsection{Motion synergies}

The results of the ANOVA tests on TPS showed that the subject's effect was non-significant $(p=.280)$ for the IMMA hand, but it was significant for the other hands, with $\mathrm{p}=.017$ for the Limbitless, $\mathrm{p}=.049$ for the Dextrus v2.0 and $\mathrm{p}=.019$ for the InMoov models. Moreover, for all the hands, the tendon pair was a significant factor on TPS.

Figure 4 shows the TPSs for each tendon pair, per subject, for all the hands analyzed. For all the hands and subjects the highest TPSs were obtained for pairs of long fingers (around $50-70 \%$ on average), showing that the subjects moved the tendons for the long fingers in a coordinated manner (CC $>0.9)$ for an important fraction of the successful grasps. The coordination between index and little fingers is always lower than that between the rest of long finger pairs. The highest coordination is generally between middle and ring fingers. The TPS value between thumb and long fingers tendons (around $20-30 \%$ on average) is lower than that observed among long fingers. Generally, the coordination between thumb and little finger flexion is lower than that between thumb and index finger flexion. For the case of the IMMA hand, the TPS values for the pair of tendons of the thumb (mean value 47\%) are on average higher than for the pairs between thumb and long fingers tendons, but lower than for long fingers' tendon pairs. 

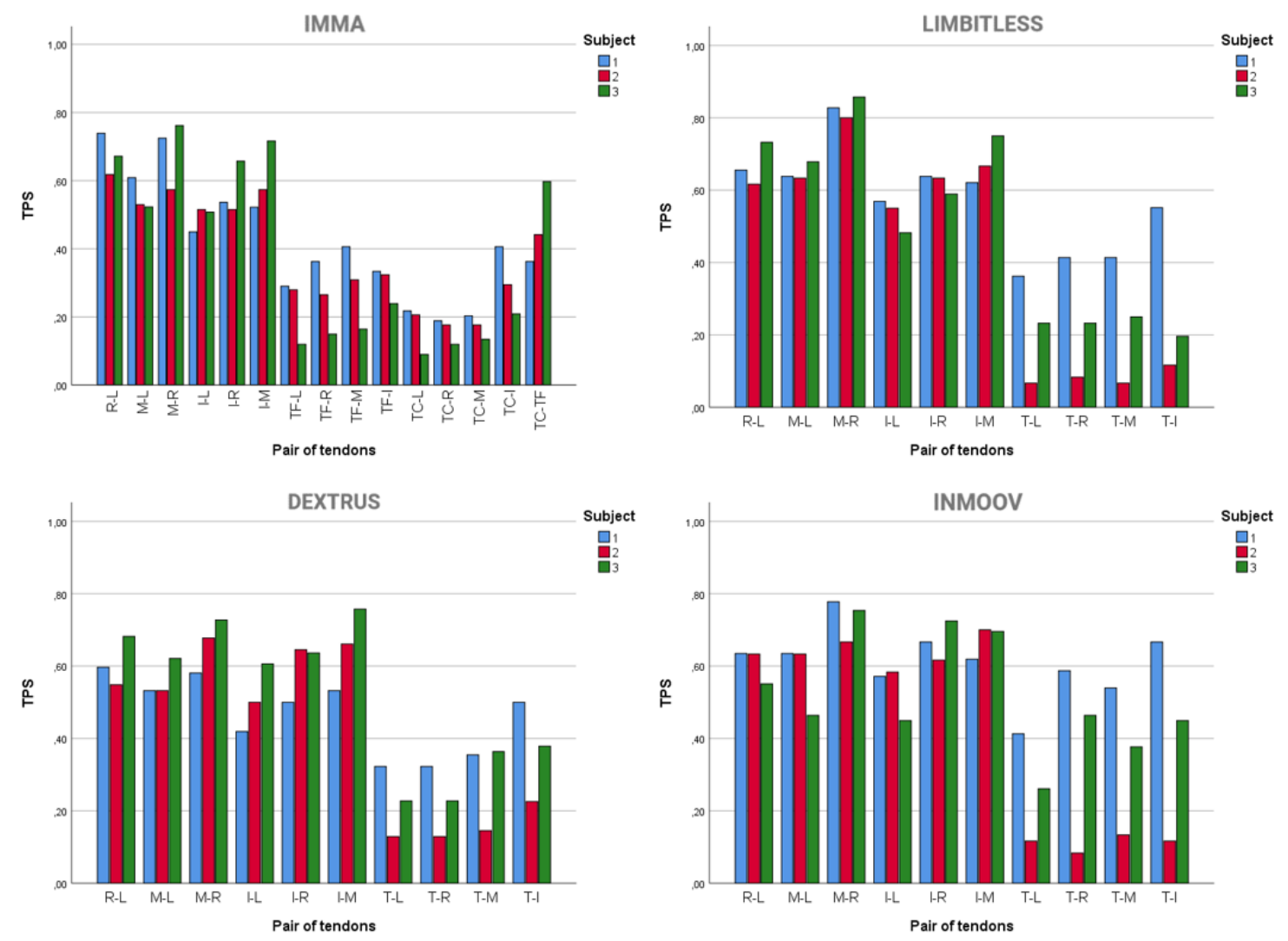

Figure 4. Tendon pair synergies (TPSs) for each pair of tendons (L: little, R: ring, M: middle, I: index, T: thumb, TF: thumb flexion, TC: thumb circumduction) during successful grasping tasks in the AHAP for the four tested hands.

The results of the PCA show that, for all the hands, two PCs explained near or more than $80 \%$ of the variance. Table 4 shows the cumulative variance explained with one or two PCs for the PCAs on the tendon displacement data matrices, both including the data for all the subjects together (Global) and for each subject independently (S1, S2, S3). Figure 5 shows the principal components (PCs) obtained. For the IMMA hand, a very similar pattern is obtained for all the subjects in coherence with the result that the subject effect on TPSs is non-significant for this hand. However, in the case of the other hands, as the subject was significant on TPSs, the PCs have small variations, especially for the index finger, depending on the subject. Generally, the first PC explains the movement of the four long fingers, where little and ring fingers have almost the same score and middle finger a little lower. The movement of the index finger is split between the first and second PCs, in different proportions depending on the hand. And the second PC represents the movement of the thumb and the remaining part of the index. 


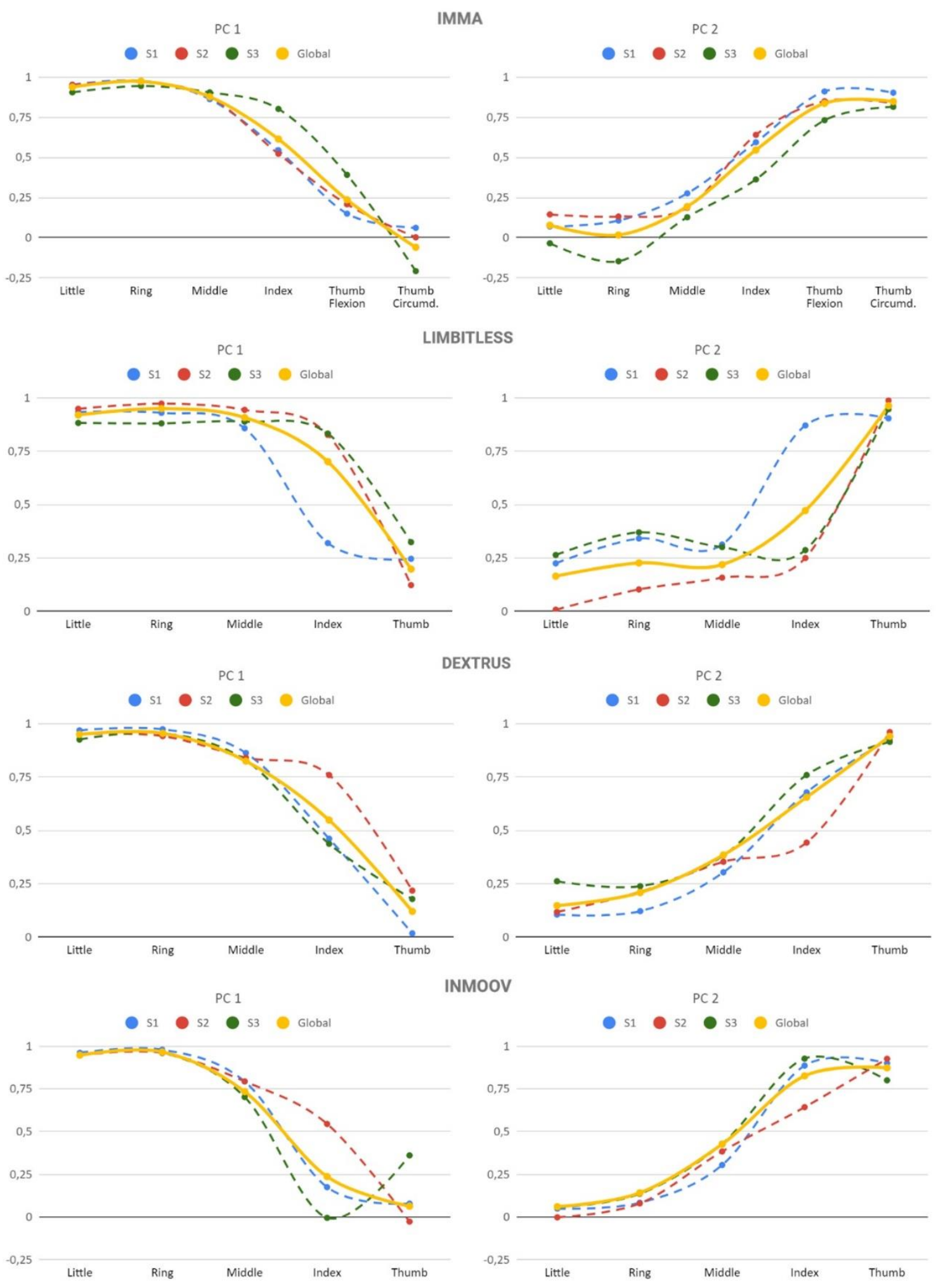

Figure 5. Eigenvectors (spline connected) for the Principal Components (PCs) of the data matrix of tendon displacements for the hands analyzed. The rotated component matrix was obtained from a PCA as extraction method and using the Varimax with Kaiser Normalization as rotation method. 


\section{4 | Chapter 3}

Table 4. Cumulative variance explained by the PCs obtained in the PCAs.

\begin{tabular}{ccccccccc}
\hline \multirow{2}{*}{ Hand } & \multicolumn{4}{c}{ PC1 (\%) } & \multicolumn{5}{c}{ PC2 (\%) } \\
& S1 & S2 & S3 & Global & S1 & S2 & S3 & Global \\
\hline IMMA & 48.8 & 48.8 & 56.1 & 50.5 & 83.6 & 80.5 & 78.9 & $\mathbf{7 9 . 8}$ \\
Limbitless & 52.6 & 68.6 & 62.8 & 62.0 & 89.4 & 90.0 & 88.2 & $\mathbf{8 7 . 5}$ \\
Dextrus & 56.7 & 62.4 & 53.5 & 56.1 & 85.5 & 88.4 & 87.1 & $\mathbf{8 6 . 6}$ \\
InMoov & 50.9 & 54.9 & 48.9 & 48.6 & 84.9 & 83.4 & 83.0 & $\mathbf{8 1 . 6}$ \\
\hline
\end{tabular}

An additional PCA with extraction method based on eigenvalue $>1$ and including the data of all the subjects (Global) resulted in just one PC only for the Limbitless and Dextrus v2.0. This PC explained $72.1 \%$ of the variance for Limbitless and 69.3\% for Dextrus v2.0. Figure 6 shows the similar pattern obtained for these two cases. Therefore, a common strategy for the transmission, actuation and control systems might be used in these hands.

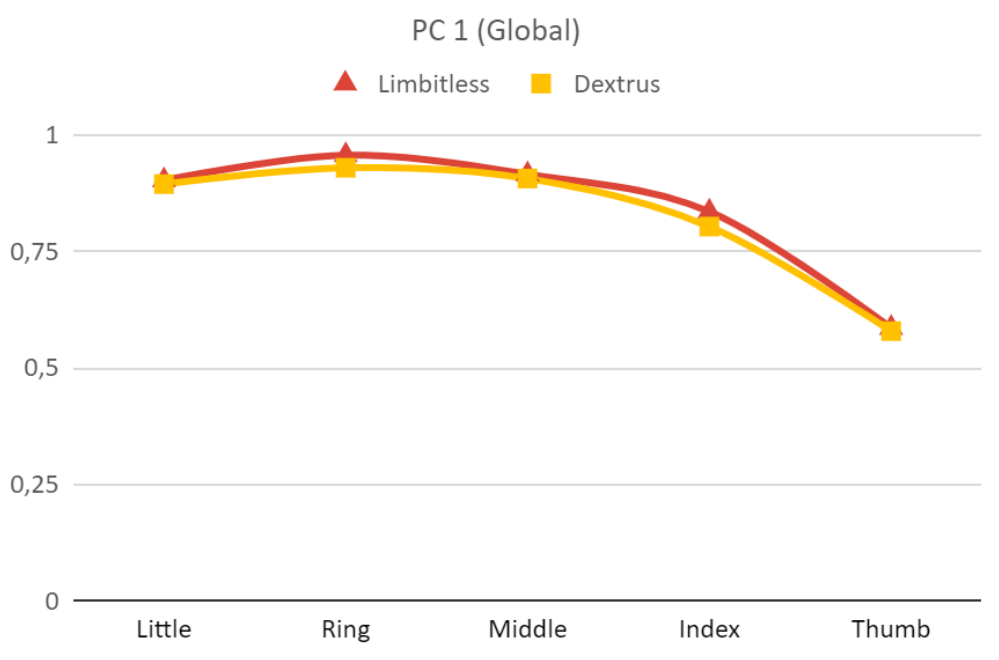

Figure 6. Eigenvectors (spline connected) for the Principal Component (PC) of the Limbitless and Dextrus v2.0 hands where the component matrix was obtained from a PCA as extraction method based on eigenvalue $>1$.

\section{Discussion}

In this study, the grasping ability of four TDPHs has been analyzed and compared using an ABA. All the tendons of the artificial hands have been actuated independently by healthy human subjects, that is, excluding from the comparison the specific control or actuation implementation with potential users with an amputation. For that reason, it is worth noting that a 
worse performance should be expected with these final users because of the less efficient feedback, control strategy, and actuation system. The objective of using this strategy was to compare the mechanical design of the hands. Thus, the differences observed in grasping ability with respect to the human hand are attributed to several factors such as a lower friction coefficient in the hand contact surfaces, the lack of dexterity because of the underactuation, or the difficulty in reaching some hand postures, including the limitations for a correct thumb opposition.

The fact that the IMMA hand scored the highest GAS is attributed mainly to two factors: it has an additional DoA for thumb circumduction and it uses selected materials for the different parts of the hand. This result seems reasonable because the IMMA hand was partially inspired by other versions of the Limbitless and Dextrus hands. The lowest GAS of the Dextrus v2.0 hand may be attributed to the use of a flexible material to generate a unibody rubberized hand, having two main consequences: the infill percentage used affects the maximum force exerted before the hand becomes warped, and the behaviour of the joints in every digit is established early in the printing process. For example, the excessive ease to bend the DIP joint on the printed model made some precision grasps cumbersome. In the other models, the replacement and customization of a joint with more infill is straightforward.

With regard to the mechanical design of the hands, several improvements should be implemented in order to increase their functionality to be able to perform the most common GTs in ADLs. They should be especially focused on improving the grasping stability because, as shown in the results, holding the object securely under motion of the hands was the most challenging matter. In the case of the EG, it would be useful to have extensor tendons acting in a complementary way or to design joints with different bending stiffness, higher for DIP than for MCP joints. These mechanical solutions may allow keeping extended the distal segments of the fingers while flexing their MCP joints. In the case of PP, LP, DVG and TP, the orientation of the thumb according to its opposition to the long fingers should be optimized. For this purpose, the authors (Pérez-González and Llop-Harillo, 2020) developed a computational method to optimize the thumb's kinematic chain (base placement, link lengths and joint orientation angles) of an artificial hand based on its performance in the Kapandji opposition test (Kapandji, 1986) used in functional evaluations of the human hand. For SG, CG and DVG, in addition to the thumb opposition, the palm opposition is also very relevant. Instead of a flat palm design, a more human-shaped one, by mimicking both thenar and hypothenar eminences, should improve the 


\section{6 | Chapter 3}

performance for these GTs. The reachable grasping force is limiting for power grasps such as DVG, EG, SG and CG, especially when grasping big and heavy objects. Decreasing the friction of the tendons along their path may improve the mechanical advantage (namely, the output to input force ratio) and thus this grasping force. Moreover, the selection of materials with good compliance and friction coefficient for the hand-objects contact areas is a key point to improve the stability of these grasps.

Regardless of the performance of the four TDPHs analyzed, the fact that the subject's effect was non-significant on both GAS and partial GAS evinces that a single subject can be used to evaluate the grasping ability of artificial hands using this ABA and the AHAP.

In this study, the motion synergies of the four TDPHs have also been analyzed and compared. The ANOVA tests on TPS showed that the subject's effect was non-significant for the IMMA hand, but it was significant for the other hands. It means that, for the case of the IMMA hand, the analyses on TPSs and PCA could be performed globally with all the data or according to one of the subjects. However, for the other hands, as the subject was found significant, differences appear, mainly in the coordination between thumb and long fingers tendons and in the scores of the PCs for the index finger.

It is relevant to highlight that the results obtained in this study on TPSs are in accordance with other studies in the literature performed with the human hand. Namely, Santello et al. (Santello et al., 1998) obtained in all subjects a high correlation between MCP angles of the long fingers, being greater for adjacent fingers. The same was also obtained for their PIP angles. Moreover, the PCA results obtained in the present paper, in which the first PC stands for the four long fingers movement and the second one mainly for the movement of the thumb, are in agreement with a recent study by GraciaIbáñez (Gracia Ibañez, 2016) where five PCs explained the synergies of the human hand performing ADLs. In that study, the first PC corresponds to the flexion of the interphalangeal joints of the fingers, and the last two PCs represent the movement of the thumb: the lateral opposition of the thumb to the index and the pad-to-pad opposition of the thumb to the little finger.

According to the information contained in Table 1 about the current design characteristics of the artificial hands, the Limbitless hand is actuated with only one motor. This actuation method is worthwhile, as one PC explains more than $70 \%$ of the variance observed for this hand. The other hands analyzed are actuated with five motors (Dextrus v2.0 and InMoov) or its actuation is not defined (IMMA). The PCs obtained in this study could be 
used for coupling the actuation for some DoFs for these hands. This underactuation is essential to simplify the control of the hand, especially for affordable designs. The actuation coupling can be implemented at the software level, with an algorithm to reproduce the PCs and/or at the hardware level, through the design of the mechanical transmission. The dimensionality reduction using only one or two motors to actuate these hands should not affect their dexterity significantly. Indeed, some of the currently existing prosthesis use only two motors, one to actuate the four long fingers and other to actuate the thumb (Weiner et al., 2018). In other cases, three motors are used (Huang et al., 2006): one for the thumb; one for the middle, ring and little finger; one for the index finger. The actuation of middle, ring and little fingers using the same motor is coherent with the results of Figure 5, as the scores corresponding to these fingers are quite similar. The use of an independent motor for the index makes sense because the score for this finger (Figure 5) has an intermediate value for the two PCs. The use of just one motor to operate all fingers carries the dimensionality reduction to its maximum (Catalano et al., 2014). The methods employed in this study could help with this dimensionality reduction and thus with the design of the underactuation in TDPHs.

\section{Conclusions}

In this study, an experimental comparison of the grasping ability of four different affordable anthropomorphic prosthetic hands has been undertaken using the AHAP benchmark. The grasping ability ranged between $48 \%$ and $57 \%$ with respect to that of the human hand, with the best result obtained by the IMMA hand, probably due to the additional DoA for circumduction of the thumb, not present in the other models, and also to the use of selected materials for the different parts of the hand. The hands exhibited better performance for non-grasping postures as index pointing or platform and for hook and tripod pinch grasps and worse performance for extension grip, pulp pinch, spherical grip, and cylindrical grip. Several factors such as the friction coefficient in the hand contact surfaces, limitations on the underactuation or for a correct thumb opposition, need to be improved in this type of prostheses, especially in relation to its grasping stability. The effect of the subject on the control of TDPHs when using an ABA has been analyzed. It has been shown that the subject's effect was non-significant on the GAS obtained, evidencing that a single subject could be used to evaluate hand prostheses using the ABA presented and the AHAP. However the motion synergies were different for some of the users and the hands analyzed, 


\section{8 | Chapter 3}

especially in the coordination between the thumb and long fingers. The analysis of the synergies in the motion of the tendons used for actuating the hands showed that the actuation and control systems could be designed in order to couple some DoFs, due to the important correlations observed. Two PCs are enough to explain more than $80 \%$ of the variability observed in the tendons motions for all the hands. The scores of these PCs can be useful for both the design of transmission systems to underactuate the hand and the design of the control system. Further research should address this underactuated design for the IMMA hand, designed by the authors, where the number of actuators could be limited to two motors while maintaining significantly the dexterity of the hand.

\section{Author Contributions}

IL designed the study and was the primary writer of the manuscript. JA and IL selected and adapted the hands to analyze. AP and IL redesigned the able-bodied adaptor. IL planned the experiments. All authors conducted the experiments. IL analyzed and interpreted the data. AP supervised the entire research process. All authors read, revised and approved the final manuscript.

\section{Funding}

This research was supported by the Spanish Ministry of Economy and Competitiveness and ESF [grant number BES-2015-076005]; the Spanish Ministry of Economy and Competitiveness, AEI and ERDF [grant numbers DPI2014-60635-R, DPI2017-89910-R]; and Universitat Jaume I, Spain [grant number UJI-B2017-70].

\section{Acknowledgements}

The authors would like to thank Jesús Cantero Ramis for his contribution in the experiments, in the electronics, and Matlab computations, and José Fuentes for his contribution in 3D printing and assembly.

\section{Data Availability Statement}

All the hands analyzed are available online under a Creative Commons license and the data can be made available from the corresponding author on reasonable request. 


\section{Ethics Statement}

The present study was approved by the Ethics Committee of the Universitat Jaume I (CD/006/2019) and written informed consent was obtained from all the participants.

\section{References}

Alec, 2015. Wounded war hero gets a 3D printed Dextrus bionic hand. https://www.3ders.org/articles/20150411-wounded-war-hero-has-beenprovided-with-a-3d-printed-dextrus-bionic-hand.html

Andrés, F.J., Pérez-González, A., Rubert, C., Fuentes, J., Sospedra, B., 2019. Comparison of Grasping Performance of Tendon and Linkage Transmission Systems in an Electric-Powered Low-Cost Hand Prosthesis. Journal of Mechanisms and Robotics, 11(1), 11018.

Asanuma, K., 2012a. Definition of the Terms "Medical Device" and "In Vitro Diagnostic (IVD) Medical Device," Global Harmonization Task Force.

Asanuma, K., 2012b. Principle of Medical Device Classification, Global Harmonization Task Force.

Bouwsema, H., van der Sluis, C.K., Bongers, R.M., 2014. Changes in performance over time while learning to use a myoelectric prosthesis. Journal of NeuroEngineering and Rehabilitation, 11(1), 16.

Bullock, I.M., Zheng, J.Z., Rosa, S.D. La, Guertler, C., Dollar, A.M., 2013. Grasp Frequency and Usage in Daily Household and Machine Shop Tasks. IEEE Transactions on Haptics, 6(3), 296-308.

Burn, M.B., Ta, A., Gogola, G.R., 2016. Three-Dimensional Printing of Prosthetic Hands for Children. The Journal of Hand Surgery, 41(5), e103-e109.

Calli, B., Walsman, A., Singh, A., Srinivasa, S., Abbeel, P., Dollar, A.M., 2015. Benchmarking in Manipulation Research: Using the Yale-CMU-Berkeley Object and Model Set. IEEE Robotics \& Automation Magazine, 22(3), 36-52.

Catalano, M.G., Grioli, G., Farnioli, E., Serio, A., Piazza, C., Bicchi, A., 2014. Adaptive synergies for the design and control of the Pisa/IIT SoftHand. The International Journal of Robotics Research, 33(5).

Controzzi, M., Cipriani, C., Carrozza, M., 2014. Design of Artificial Hands: A Review., in: Balasubramanian, R., Santos, V. (Eds.), The Human Hand as an Inspiration for Robot Hand Development. Springer, Cham, pp. 219-246.

Dalley, S.A., Bennett, D.A., Goldfarb, M., 2012. Preliminary functional assessment of a multigrasp myoelectric prosthesis, in: 2012 Annual International Conference of the IEEE Engineering in Medicine and Biology Society. IEEE, pp. 4172-4175. 
Dally, C., Johnson, D., Canon, M., Ritter, S., Mehta, K., 2015. Characteristics of a 3D-printed prosthetic hand for use in developing countries, in: Proceedings of the 5th IEEE Global Humanitarian Technology Conference, GHTC 2015. pp. 66-70.

e-NABLE, 2014. http://enablingthefuture.org/which-design/

Feix, T., Romero, J., Schmiedmayer, H.-B., Dollar, A.M., Kragic, D., 2016. The GRASP Taxonomy of Human Grasp Types. IEEE Transactions on HumanMachine Systems, 46(1), 66-77.

Fougner, A.L., Stavdahl, Ø., Kyberd, P.J., 2014. System training and assessment in simultaneous proportional myoelectric prosthesis control. Journal of NeuroEngineering and Rehabilitation, 11(1), 75.

Gibbard, J., 2013. Open Hand Project. www.openhandproject.org

Gibbard, J., 2018. Openbionics. https://www.openbionics.com

Gibbard, J., 2015. Open Hand Project: Downloads. http://www.openhandproject.org/downloads.php

Gracia Ibañez, V., 2016. Contribution to hand functional assessment based on its kinematics. Universitat Jaume I, Castelló de la Plana.

Huang, H., Jiang, L., Zhao, D.W., Zhao, J.D., Cai, H.G., Liu, H., Meusel, P., Willberg, B., Hirzinger, G., 2006. The development on a new biomechatronic prosthetic hand based on under-actuated mechanism, in: IEEE International Conference on Intelligent Robots and Systems. pp. 3791-3796.

Huchet, N., 2014. Bionicohand: Open Source Myohand. https://bionico.org/

Huinink, L.H.B., Bouwsema, H., Plettenburg, D.H., van der Sluis, C.K., Bongers, R.M., 2016. Learning to use a body-powered prosthesis: changes in functionality and kinematics. Journal of NeuroEngineering and Rehabilitation, 13(1), 90.

Kapandji, A., 1986. Clinical opposition and reposition test of the thumb [COTATION CLINIQUE DE L'OPPOSITION ET DE LA CONTREOPPOSITION DU POUCE]. Annales de Chirurgie de la Main, 5(1), 67-73.

Kyberd, P.J., 2011. The influence of control format and hand design in single axis myoelectric hands: assessment of functionality of prosthetic hands using the Southampton Hand Assessment Procedure. Prosthetics and Orthotics International, 35(3), 285-293.

Langevin, G., 2013. Inmoov Hand and Forearm. http://inmoov.fr/hand-andforarm/

Light, C.M., Chappell, P.H., Kyberd, P.J., 2002. Establishing a standardized clinical assessment tool of pathologic and prosthetic hand function: Normative data, reliability, and validity. Archives of Physical Medicine and Rehabilitation, 83(6), 776-783. 
Limbitless solutions, 2015. https://limbitless-solutions.org/

Lindner, H.Y.N., Nätterlund, B.S., Hermansson, L.M.N., 2010. Upper limb prosthetic outcome measures: review and content comparison based on International Classification of Functioning, Disability and Health. Prosthetics and orthotics international, 34(2), 109-128.

Llop-Harillo, I., Pérez-González, A., 2017a. System for the experimental evaluation of anthropomorphic hands. Application to a new 3D-printed prosthetic hand prototype. International Biomechanics, 4(2), 50-59.

Llop-Harillo, I., Pérez-González, A., 2017b. IMMA hand (Devalhand project). https://sites.google.com/a/uji.es/devalhand/imma-hand

Llop-Harillo, I., Pérez-González, A., Starke, J., Asfour, T., 2019. The Anthropomorphic Hand Assessment Protocol (AHAP). Robotics and Autonomous Systems, 121.

Mathiowetz, V., Volland, G., Kashman, N., Weber, K., 1985. Adult Norms for the Box and Block Test of Manual Dexterity. American Journal of Occupational Therapy, 39(6), 386-391.

Mathiowetz, V., Weber, K., Kashman, N., Volland, G., 1985. Adult norms for the Nine Hole Peg Test of finger dexterity. Occupational Therapy Journal of Research.

Owen, J., 2018. Limbitless Solutions Begin Clinical Trials For 3D Printed Arms. https://enablingthefuture.org/2018/05/30/limbitless-solutions-begin-clinicaltrials-for-3d-printed-arms/

Pérez-González, A., Llop-Harillo, I., 2020. Optimization of the Kinematic Chain of the Thumb for a Hand Prosthesis Based on the Kapandji Opposition Test. In: Ateshian G., Myers K., Tavares J. (eds) Computer Methods, Imaging and Visualization in Biomechanics and Biomedical Engineering. CMBBE 2019. Lecture Notes in Computational Vision and Biomechanics, vol 36. Springer, Cham.

Phillips, B., Zingalis, G., Ritter, S., Mehta, K., 2015. A review of current upperlimb prostheses for resource constrained settings, in: 2015 IEEE Global Humanitarian Technology Conference (GHTC). IEEE, pp. 52-58.

RepRap, 2017. http://reprap.org/

Rossi, M., Della Santina, C., Piazza, C., Grioli, G., Catalano, M., Bicchi, A., 2017. Preliminary results toward a naturally controlled multi-synergistic prosthetic hand, in: 2017 International Conference on Rehabilitation Robotics (ICORR). IEEE, pp. 1356-1363.

Salvietti, G., 2018. Replicating Human Hand Synergies Onto Robotic Hands: A Review on Software and Hardware Strategies. Frontiers in Neurorobotics, 12(JUN). 
Santello, M., Bianchi, M., Gabiccini, M., Ricciardi, E., Salvietti, G., Prattichizzo, D., Ernst, M., Moscatelli, A., Jörntell, H., Kappers, A.M.L., Kyriakopoulos, K., Albu-Schäffer, A., Castellini, C., Bicchi, A., 2016. Hand synergies: Integration of robotics and neuroscience for understanding the control of biological and artificial hands. Physics of Life Reviews, 17, 1-23.

Santello, M., Flanders, M., Soechting, J.F., 1998. Postural hand synergies for tool use. The Journal of neuroscience: the official journal of the Society for Neuroscience, 18(23), 10105-10115.

Smit, G., Plettenburg, D., Van der Helm, F., 2015. The Lightweight Delft Cylinder Hand, the First Multi-Articulating Hand That Meets the Basic User Requirements. IEEE Transactions on Neural Systems and Rehabilitation Engineering, 23(3), 431-440.

Sollerman, C., Ejeskär, A., 1995. Sollerman Hand Function Test: A Standardised Method and its Use in Tetraplegic Patients. Scandinavian Journal of Plastic and Reconstructive Surgery and Hand Surgery, 29(2), 167-176.

Steve Wood, 2014. The Flexy Hand and Flexy Hand 2. http://enablingthefuture.org/upper-limb-prosthetics/the-flexy-hand/ [9.20.17].

Tanaka, K.S., Lightdale-Miric, N., 2016. Advances in 3D-Printed Pediatric Prostheses for Upper Extremity Differences. The Journal of Bone and Joint Surgery, 98(15), 1320-1326.

ten Kate, J., Smit, G., Breedveld, P., 2017. 3D-printed upper limb prostheses: a review. Disability and Rehabilitation: Assistive Technology.

U.S. Department of Health and Human Services - National Institutes of Health, 2017. 3dprint.nih.gov

UCFArmory, Enablingthefuture, 2014. Limbitless arm. https://www.thingiverse.com/thing:408641

Vasluian, E., Bongers, R.M., Reinders-Messelink, H.A., Burgerhof, J.G.M., Dijkstra, P.U., Sluis, C., 2014. Learning effects of repetitive administration of the Southampton Hand Assessment Procedure in novice prosthetic users. Journal of Rehabilitation Medicine, 46(8), 788-797.

Ventola, C.L., 2014. Medical Applications for 3D Printing: Current and Projected Uses. P \& T: a peer-reviewed journal for formulary management, 39(10), 704-711.

Vergara, M., Agost, M.J., Gracia-Ibáñez, V., 2018. Dorsal and palmar aspect dimensions of hand anthropometry for designing hand tools and protections. Human Factors and Ergonomics in Manufacturing \& Service Industries.

Vergara, M., Sancho-Bru, J.L., Gracia-Ibáñez, V., Pérez-González, A., 2014. An introductory study of common grasps used by adults during performance of activities of daily living. Journal of Hand Therapy, 27(3), 225-234. 
Wang, S., Hsu, C.J., Trent, L., Ryan, T., Kearns, N.T., Civillico, E.F., Kontson, K.L., 2018. Evaluation of Performance-Based Outcome Measures for the Upper Limb: A Comprehensive Narrative Review. $P M \& R, 10(9), 951-$ 962.e3.

Weiner, P., Starke, J., Hundhausen, F., Beil, J., Asfour, T., 2018. The KIT Prosthetic Hand: Design and Control, in: 2018 IEEE/RSJ International Conference on Intelligent Robots and Systems (IROS). IEEE, pp. 3328-3334.

\section{Supplementary Material}

Supplementary Table 1. Scores for each step of each repetition of the different tasks of the AHAP obtained for each hand analyzed and each subject.

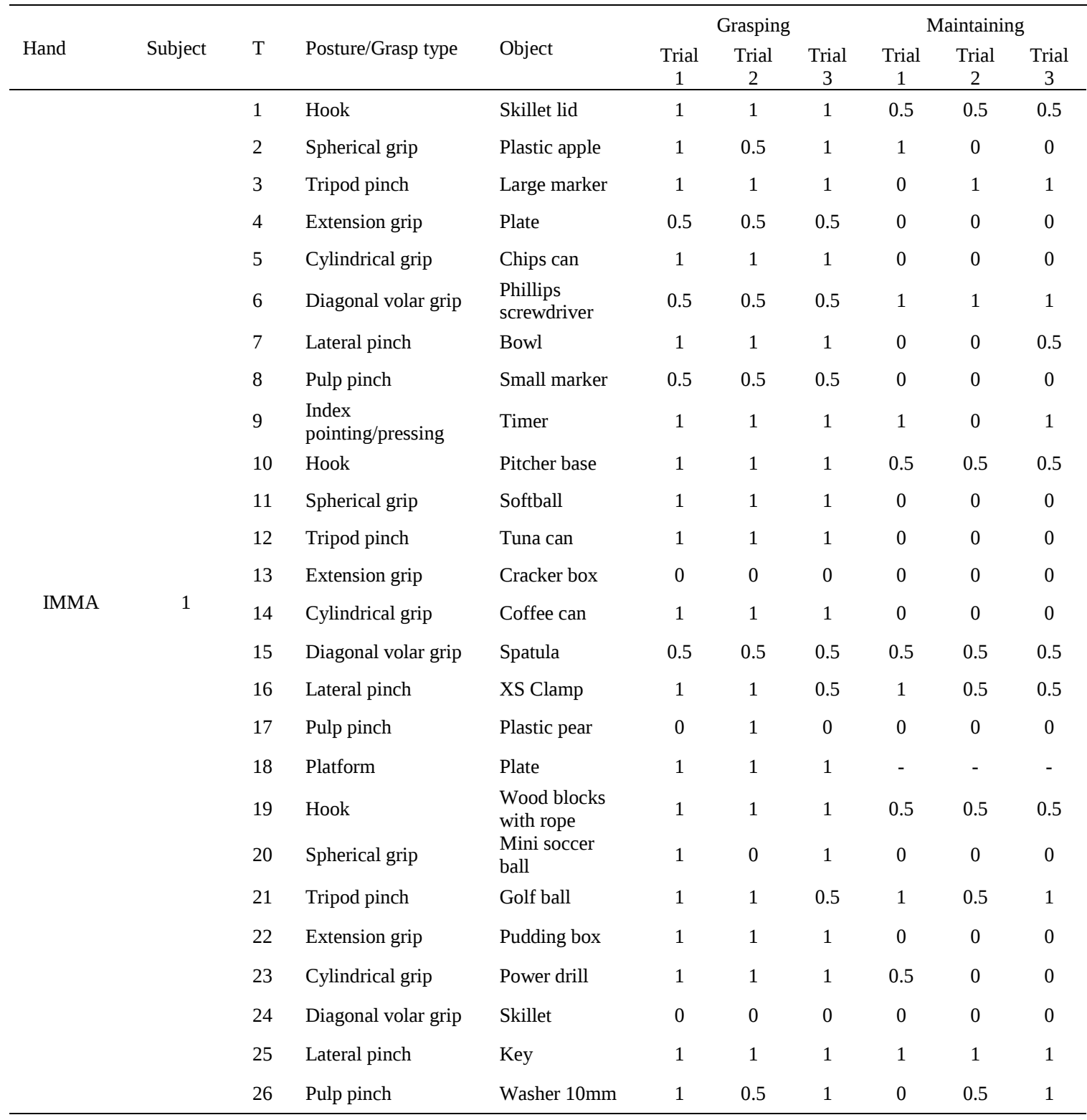




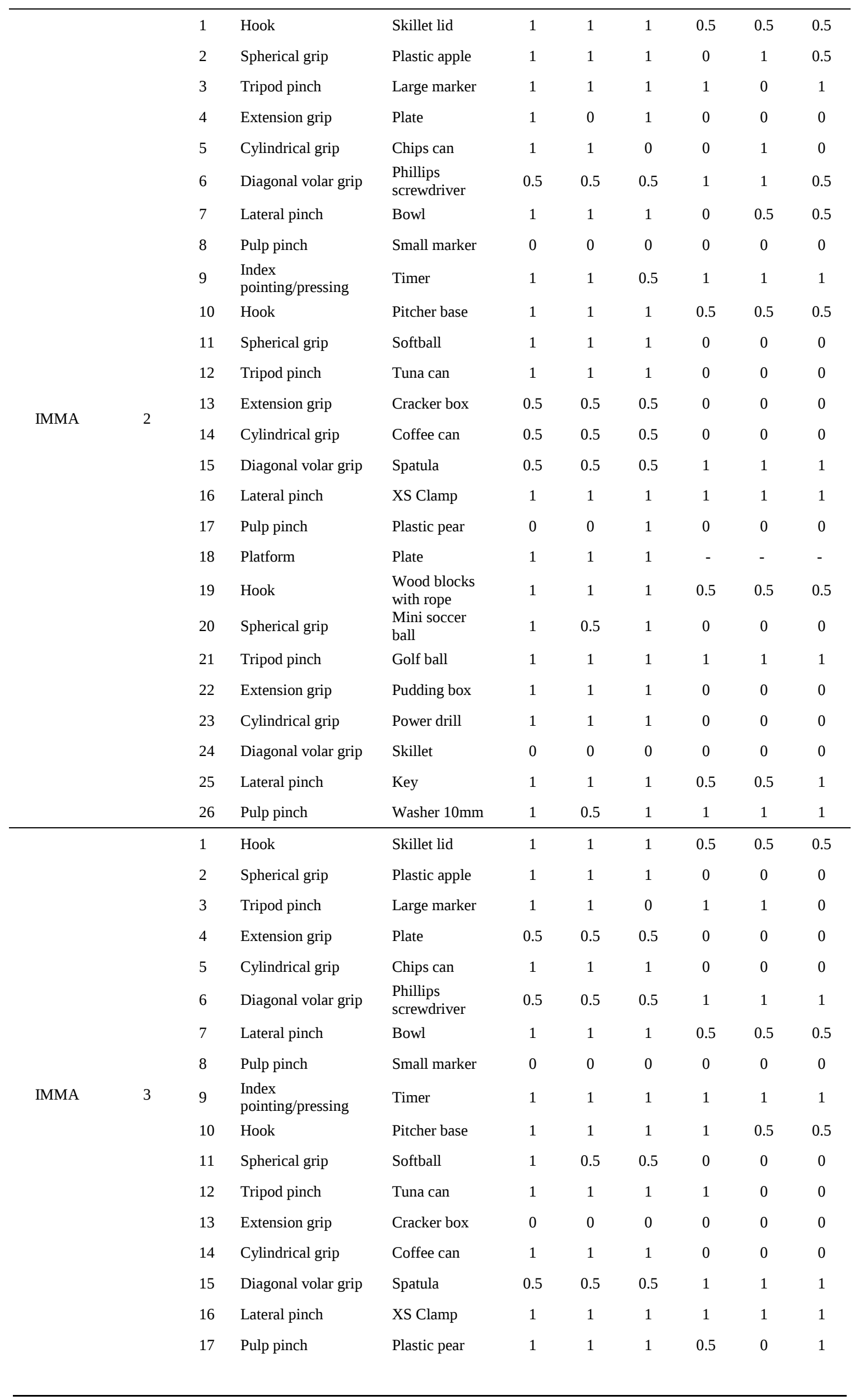




\begin{tabular}{|c|c|c|c|c|c|c|c|c|c|c|}
\hline & & 18 & Platform & Plate & 1 & 1 & 1 & - & - & - \\
\hline & & 19 & Hook & $\begin{array}{l}\text { Wood blocks } \\
\text { with rope }\end{array}$ & 1 & 1 & 1 & 0.5 & 0.5 & 0.5 \\
\hline & & 20 & Spherical grip & $\begin{array}{l}\text { Mini soccer } \\
\text { ball }\end{array}$ & 1 & 0.5 & 0.5 & 0 & 0 & 0 \\
\hline & & 21 & Tripod pinch & Golf ball & 1 & 1 & 0.5 & 1 & 1 & 1 \\
\hline & & 22 & Extension grip & Pudding box & 1 & 0.5 & 1 & 0 & 0 & 0 \\
\hline & & 23 & Cylindrical grip & Power drill & 1 & 1 & 1 & 0 & 0 & 0 \\
\hline & & 24 & Diagonal volar grip & Skillet & 0 & 0 & 0 & 0 & 0 & 0 \\
\hline & & 25 & Lateral pinch & Key & 1 & 1 & 1 & 0.5 & 1 & 1 \\
\hline & & 26 & Pulp pinch & Washer 10mm & 1 & 0 & 0.5 & 0 & 0 & 1 \\
\hline \multirow{26}{*}{ Limbitless } & \multirow{26}{*}{1} & 1 & Hook & Skillet lid & 1 & 1 & 1 & 0.5 & 0.5 & 0.5 \\
\hline & & 2 & Spherical grip & Plastic apple & 0 & 1 & 1 & 0 & 0 & 0 \\
\hline & & 3 & Tripod pinch & Large marker & 0 & 1 & 0 & 0 & 0 & 0 \\
\hline & & 4 & Extension grip & Plate & 1 & 1 & 1 & 0 & 0 & 0 \\
\hline & & 5 & Cylindrical grip & Chips can & 0.5 & 0.5 & 0.5 & 0 & 0 & 0 \\
\hline & & 6 & Diagonal volar grip & $\begin{array}{l}\text { Phillips } \\
\text { screwdriver }\end{array}$ & 0.5 & 0.5 & 0.5 & 1 & 1 & 1 \\
\hline & & 7 & Lateral pinch & Bowl & 1 & 1 & 1 & 0.5 & 0.5 & 0 \\
\hline & & 8 & Pulp pinch & Small marker & 0 & 0 & 0 & 0 & 0 & 0 \\
\hline & & 9 & $\begin{array}{l}\text { Index } \\
\text { pointing/pressing }\end{array}$ & Timer & 1 & 1 & 1 & 1 & 1 & 1 \\
\hline & & 10 & Hook & Pitcher base & 1 & 1 & 1 & 0.5 & 0.5 & 0.5 \\
\hline & & 11 & Spherical grip & Softball & 1 & 1 & 1 & 0 & 0 & 0 \\
\hline & & 12 & Tripod pinch & Tuna can & 1 & 1 & 1 & 0 & 0.5 & 0 \\
\hline & & 13 & Extension grip & Cracker box & 0 & 0 & 0 & 0 & 0 & 0 \\
\hline & & 14 & Cylindrical grip & Coffee can & 0.5 & 0.5 & 0.5 & 0 & 0 & 0 \\
\hline & & 15 & Diagonal volar grip & Spatula & 0.5 & 0.5 & 0.5 & 1 & 1 & 1 \\
\hline & & 16 & Lateral pinch & XS Clamp & 0.5 & 0.5 & 0.5 & 1 & 1 & 1 \\
\hline & & 17 & Pulp pinch & Plastic pear & 0 & 0.5 & 0 & 0 & 1 & 0 \\
\hline & & 18 & Platform & Plate & 0 & 0 & 0 & - & - & - \\
\hline & & 19 & Hook & $\begin{array}{l}\text { Wood blocks } \\
\text { with rope }\end{array}$ & 1 & 1 & 1 & 0.5 & 0.5 & 0.5 \\
\hline & & 20 & Spherical grip & $\begin{array}{l}\text { Mini soccer } \\
\text { ball }\end{array}$ & 0 & 0 & 0 & 0 & 0 & 0 \\
\hline & & 21 & Tripod pinch & Golf ball & 1 & 1 & 1 & 1 & 1 & 0 \\
\hline & & 22 & Extension grip & Pudding box & 1 & 1 & 1 & 0 & 0 & 0 \\
\hline & & 23 & Cylindrical grip & Power drill & 1 & 1 & 1 & 1 & 0.5 & 0.5 \\
\hline & & 24 & Diagonal volar grip & Skillet & 0.5 & 0.5 & 0.5 & 0 & 0 & 0 \\
\hline & & 25 & Lateral pinch & Key & 1 & 1 & 1 & 1 & 1 & 0.5 \\
\hline & & 26 & Pulp pinch & Washer 10mm & 0 & 0 & 0 & 0 & 0 & 0 \\
\hline \multirow{8}{*}{ Limbitless } & \multirow{8}{*}{2} & 1 & Hook & Skillet lid & 1 & 1 & 1 & 0.5 & 0.5 & 1 \\
\hline & & 2 & Spherical grip & Plastic apple & 0 & 0 & 0 & 0 & 0 & 0 \\
\hline & & 3 & Tripod pinch & Large marker & 0 & 1 & 1 & 0 & 1 & 1 \\
\hline & & 4 & Extension grip & Plate & 1 & 1 & 1 & 0 & 0 & 0 \\
\hline & & 5 & Cylindrical grip & Chips can & 0.5 & 0.5 & 0.5 & 0 & 0 & 0 \\
\hline & & 6 & Diagonal volar grip & $\begin{array}{l}\text { Phillips } \\
\text { screwdriver }\end{array}$ & 0.5 & 0.5 & 0.5 & 1 & 1 & 1 \\
\hline & & 7 & Lateral pinch & Bowl & 1 & 1 & 1 & 0.5 & 0.5 & 1 \\
\hline & & 8 & Pulp pinch & Small marker & 0 & 0 & 0 & 0 & 0 & 0 \\
\hline
\end{tabular}




\begin{tabular}{|c|c|c|c|c|c|c|c|c|c|c|}
\hline & & \multirow{2}{*}{$\begin{array}{l}9 \\
10\end{array}$} & \multirow{2}{*}{$\begin{array}{l}\text { Index } \\
\text { pointing/pressing } \\
\text { Hook }\end{array}$} & \multirow{2}{*}{$\begin{array}{l}\text { Timer } \\
\text { Pitcher base }\end{array}$} & \multirow{2}{*}{$\begin{array}{l}1 \\
1\end{array}$} & \multirow{2}{*}{$\begin{array}{l}1 \\
1\end{array}$} & \multirow{2}{*}{$\begin{array}{l}1 \\
1\end{array}$} & \multirow{2}{*}{$\begin{array}{l}1 \\
1\end{array}$} & \multirow{2}{*}{$\begin{array}{c}1 \\
0.5\end{array}$} & \multirow{2}{*}{$\begin{array}{c}1 \\
0.5\end{array}$} \\
\hline & & & & & & & & & & \\
\hline & & 11 & Spherical grip & Softball & 1 & 1 & 1 & 0 & 0 & 0 \\
\hline & & 12 & Tripod pinch & Tuna can & 1 & 1 & 1 & 0 & 0 & 0 \\
\hline & & 13 & Extension grip & Cracker box & 0 & 0 & 0 & 0 & 0 & 0 \\
\hline & & 14 & Cylindrical grip & Coffee can & 0.5 & 0.5 & 0.5 & 0 & 0 & 0 \\
\hline & & 15 & Diagonal volar grip & Spatula & 0.5 & 0.5 & 0.5 & 1 & 1 & 1 \\
\hline & & 16 & Lateral pinch & XS Clamp & 0 & 0 & 0.5 & 0 & 0 & 1 \\
\hline & & 17 & Pulp pinch & Plastic pear & 0.5 & 0.5 & 0.5 & 0.5 & 0 & 1 \\
\hline & & 18 & Platform & Plate & 0 & 0 & 0 & - & - & - \\
\hline & & 19 & Hook & $\begin{array}{l}\text { Wood blocks } \\
\text { with rope }\end{array}$ & 1 & 1 & 1 & 0.5 & 0.5 & 0.5 \\
\hline & & 20 & Spherical grip & $\begin{array}{l}\text { Mini soccer } \\
\text { ball }\end{array}$ & 0 & 0 & 0 & 0 & 0 & 0 \\
\hline & & 21 & Tripod pinch & Golf ball & 1 & 1 & 1 & 1 & 1 & 1 \\
\hline & & 22 & Extension grip & Pudding box & 1 & 1 & 1 & 0 & 0.5 & 0 \\
\hline & & 23 & Cylindrical grip & Power drill & 1 & 1 & 1 & 1 & 0.5 & 1 \\
\hline & & 24 & Diagonal volar grip & Skillet & 0.5 & 0.5 & 0.5 & 0 & 0 & 0 \\
\hline & & 25 & Lateral pinch & Key & 1 & 1 & 1 & 1 & 0.5 & 0.5 \\
\hline & & 26 & Pulp pinch & Washer 10mm & 1 & 1 & 1 & 1 & 1 & 0.5 \\
\hline \multirow{25}{*}{ Limbitless } & \multirow{25}{*}{3} & 1 & Hook & Skillet lid & 1 & 1 & 1 & 0.5 & 0.5 & 0.5 \\
\hline & & 2 & Spherical grip & Plastic apple & 0 & 1 & 1 & 0 & 0 & 0 \\
\hline & & 3 & Tripod pinch & Large marker & 1 & 0 & 1 & 1 & 0 & 1 \\
\hline & & 4 & Extension grip & Plate & 1 & 1 & 1 & 0 & 0 & 0 \\
\hline & & 5 & Cylindrical grip & Chips can & 0.5 & 0.5 & 0.5 & 0 & 0 & 0 \\
\hline & & 6 & Diagonal volar grip & $\begin{array}{l}\text { Phillips } \\
\text { screwdriver }\end{array}$ & 0.5 & 0.5 & 0.5 & 1 & 1 & 1 \\
\hline & & 7 & Lateral pinch & Bowl & 1 & 1 & 1 & 0 & 0 & 0 \\
\hline & & 8 & Pulp pinch & Small marker & 0 & 0 & 0 & 0 & 0 & 0 \\
\hline & & 9 & $\begin{array}{l}\text { Index } \\
\text { pointing/pressing }\end{array}$ & Timer & 1 & 1 & 1 & 1 & 1 & 1 \\
\hline & & 10 & Hook & Pitcher base & 1 & 1 & 1 & 1 & 0.5 & 1 \\
\hline & & 11 & Spherical grip & Softball & 1 & 1 & 1 & 0 & 0 & 0 \\
\hline & & 12 & Tripod pinch & Tuna can & 1 & 1 & 1 & 0 & 0 & 0.5 \\
\hline & & 13 & Extension grip & Cracker box & 0 & 0 & 0 & 0 & 0 & 0 \\
\hline & & 14 & Cylindrical grip & Coffee can & 0.5 & 0.5 & 0.5 & 0 & 0 & 0 \\
\hline & & 15 & Diagonal volar grip & Spatula & 0.5 & 0.5 & 0.5 & 1 & 1 & 1 \\
\hline & & 16 & Lateral pinch & XS Clamp & 0 & 0 & 0 & 0 & 0 & 0 \\
\hline & & 17 & Pulp pinch & Plastic pear & 0.5 & 0.5 & 0.5 & 1 & 0 & 0 \\
\hline & & 18 & Platform & Plate & 0 & 0 & 0 & - & - & - \\
\hline & & 19 & Hook & $\begin{array}{l}\text { Wood blocks } \\
\text { with rope }\end{array}$ & 1 & 1 & 1 & 0.5 & 0.5 & 1 \\
\hline & & 20 & Spherical grip & $\begin{array}{l}\text { Mini soccer } \\
\text { ball }\end{array}$ & 0 & 0 & 0 & 0 & 0 & 0 \\
\hline & & 21 & Tripod pinch & Golf ball & 1 & 1 & 1 & 1 & 1 & 1 \\
\hline & & 22 & Extension grip & Pudding box & 1 & 1 & 1 & 0 & 0 & 0 \\
\hline & & 23 & Cylindrical grip & Power drill & 1 & 1 & 1 & 0.5 & 0.5 & 1 \\
\hline & & 24 & Diagonal volar grip & Skillet & 0 & 0.5 & 0 & 0 & 0 & 0 \\
\hline & & 25 & Lateral pinch & Key & 1 & 1 & 1 & 1 & 1 & 1 \\
\hline
\end{tabular}


Chapter 3 | 137

\begin{tabular}{|c|c|c|c|c|c|c|c|c|c|c|}
\hline & & 26 & Pulp pinch & Washer 10mm & 0 & 0 & 0 & 0 & 0 & 0 \\
\hline \multirow{26}{*}{$\begin{array}{l}\text { Dextrus } \\
\text { v2.0 }\end{array}$} & \multirow{26}{*}{1} & 1 & Hook & Skillet lid & 1 & 1 & 1 & 0.5 & 0.5 & 0.5 \\
\hline & & 2 & Spherical grip & Plastic apple & 0.5 & 0.5 & 1 & 0 & 1 & 0 \\
\hline & & 3 & Tripod pinch & Large marker & 1 & 1 & 1 & 1 & 0 & 1 \\
\hline & & 4 & Extension grip & Plate & 0 & 0.5 & 0 & 0 & 0 & 0 \\
\hline & & 5 & Cylindrical grip & Chips can & 1 & 1 & 1 & 0 & 0 & 0 \\
\hline & & 6 & Diagonal volar grip & $\begin{array}{l}\text { Phillips } \\
\text { screwdriver }\end{array}$ & 0.5 & 0.5 & 0.5 & 1 & 1 & 1 \\
\hline & & 7 & Lateral pinch & Bowl & 0.5 & 1 & 0.5 & 0 & 0 & 0 \\
\hline & & 8 & Pulp pinch & Small marker & 0 & 0 & 0.5 & 0 & 0 & 0.5 \\
\hline & & 9 & $\begin{array}{l}\text { Index } \\
\text { pointing/pressing }\end{array}$ & Timer & 1 & 1 & 1 & 1 & 1 & 1 \\
\hline & & 10 & Hook & Pitcher base & 1 & 1 & 1 & 0.5 & 1 & 1 \\
\hline & & 11 & Spherical grip & Softball & 0.5 & 0.5 & 1 & 0 & 0 & 0 \\
\hline & & 12 & Tripod pinch & Tuna can & 1 & 0 & 0.5 & 0 & 0 & 0 \\
\hline & & 13 & Extension grip & Cracker box & 0 & 0 & 0 & 0 & 0 & 0 \\
\hline & & 14 & Cylindrical grip & Coffee can & 0.5 & 0.5 & 0.5 & 0 & 0 & 0 \\
\hline & & 15 & Diagonal volar grip & Spatula & 0.5 & 0.5 & 0.5 & 1 & 1 & 0.5 \\
\hline & & 16 & Lateral pinch & XS Clamp & 0.5 & 0.5 & 0.5 & 0.5 & 0.5 & 0.5 \\
\hline & & 17 & Pulp pinch & Plastic pear & 0 & 0 & 0.5 & 0 & 0 & 0 \\
\hline & & 18 & Platform & Plate & 1 & 1 & 1 & - & - & - \\
\hline & & 19 & Hook & $\begin{array}{l}\text { Wood blocks } \\
\text { with rope }\end{array}$ & 1 & 1 & 1 & 0.5 & 0.5 & 1 \\
\hline & & 20 & Spherical grip & $\begin{array}{l}\text { Mini soccer } \\
\text { ball }\end{array}$ & 0.5 & 0.5 & 0.5 & 0 & 0 & 0 \\
\hline & & 21 & Tripod pinch & Golf ball & 1 & 1 & 1 & 0.5 & 1 & 1 \\
\hline & & 22 & Extension grip & Pudding box & 0.5 & 0.5 & 0 & 0 & 0 & 0 \\
\hline & & 23 & Cylindrical grip & Power drill & 1 & 0.5 & 0.5 & 0 & 0 & 0 \\
\hline & & 24 & Diagonal volar grip & Skillet & 0 & 0 & 0 & 0 & 0 & 0 \\
\hline & & 25 & Lateral pinch & Key & 0 & 0 & 0.5 & 0 & 0 & 0 \\
\hline & & 26 & Pulp pinch & Washer 10mm & 0.5 & 0.5 & 0.5 & 0 & 0 & 0 \\
\hline \multirow{16}{*}{$\begin{array}{l}\text { Dextrus } \\
\text { v2.0 }\end{array}$} & \multirow{16}{*}{2} & 1 & Hook & Skillet lid & 1 & 1 & 1 & 0.5 & 0.5 & 0.5 \\
\hline & & 2 & Spherical grip & Plastic apple & 1 & 1 & 1 & 0 & 0 & 0 \\
\hline & & 3 & Tripod pinch & Large marker & 1 & 0 & 0.5 & 1 & 0 & 1 \\
\hline & & 4 & Extension grip & Plate & 0.5 & 1 & 0.5 & 0 & 0 & 0 \\
\hline & & 5 & Cylindrical grip & Chips can & 1 & 1 & 1 & 0 & 0 & 0 \\
\hline & & 6 & Diagonal volar grip & $\begin{array}{l}\text { Phillips } \\
\text { screwdriver }\end{array}$ & 0.5 & 0.5 & 0.5 & 1 & 1 & 1 \\
\hline & & 7 & Lateral pinch & Bowl & 1 & 1 & 1 & 0 & 0 & 0 \\
\hline & & 8 & Pulp pinch & Small marker & 0 & 0 & 0 & 0 & 0 & 0 \\
\hline & & 9 & $\begin{array}{l}\text { Index } \\
\text { pointing/pressing }\end{array}$ & Timer & 0 & 0 & 1 & 0 & 0 & 0 \\
\hline & & 10 & Hook & Pitcher base & 1 & 1 & 1 & 0.5 & 0.5 & 0.5 \\
\hline & & 11 & Spherical grip & Softball & 0.5 & 0 & 0.5 & 0 & 0 & 0 \\
\hline & & 12 & Tripod pinch & Tuna can & 1 & 1 & 1 & 0 & 0 & 0 \\
\hline & & 13 & Extension grip & Cracker box & 0 & 0 & 0 & 0 & 0 & 0 \\
\hline & & 14 & Cylindrical grip & Coffee can & 1 & 1 & 1 & 0 & 0 & 0 \\
\hline & & 15 & Diagonal volar grip & Spatula & 0.5 & 0.5 & 0.5 & 1 & 1 & 1 \\
\hline & & 16 & Lateral pinch & XS Clamp & 0.5 & 0.5 & 0.5 & 0 & 1 & 1 \\
\hline
\end{tabular}




\begin{tabular}{|c|c|c|c|c|c|c|c|c|c|c|}
\hline & & 17 & Pulp pinch & Plastic pear & 0.5 & 0.5 & 0.5 & 0 & 0 & 0 \\
\hline & & 18 & Platform & Plate & 1 & 1 & 1 & - & - & - \\
\hline & & 19 & Hook & $\begin{array}{l}\text { Wood blocks } \\
\text { with rope }\end{array}$ & 1 & 1 & 1 & 0.5 & 0.5 & 0.5 \\
\hline & & 20 & Spherical grip & $\begin{array}{l}\text { Mini soccer } \\
\text { ball }\end{array}$ & 0 & 0.5 & 0 & 0 & 0 & 0 \\
\hline & & 21 & Tripod pinch & Golf ball & 1 & 1 & 1 & 1 & 1 & 1 \\
\hline & & 22 & Extension grip & Pudding box & 0.5 & 0.5 & 0.5 & 1 & 1 & 1 \\
\hline & & 23 & Cylindrical grip & Power drill & 0.5 & 0.5 & 0.5 & 0 & 0 & 0 \\
\hline & & 24 & Diagonal volar grip & Skillet & 0 & 0 & 0 & 0 & 0 & 0 \\
\hline & & 25 & Lateral pinch & Key & 0 & 0.5 & 1 & 0 & 0.5 & 0 \\
\hline & & 26 & Pulp pinch & Washer 10mm & 0.5 & 0.5 & 1 & 1 & 1 & 1 \\
\hline \multirow{26}{*}{$\begin{array}{c}\text { Dextrus } \\
\text { v2.0 }\end{array}$} & \multirow{26}{*}{3} & 1 & Hook & Skillet lid & 1 & 1 & 1 & 0.5 & 0.5 & 0.5 \\
\hline & & 2 & Spherical grip & Plastic apple & 0.5 & 0.5 & 0.5 & 1 & 0 & 1 \\
\hline & & 3 & Tripod pinch & Large marker & 0 & 1 & 1 & 0 & 1 & 1 \\
\hline & & 4 & Extension grip & Plate & 0.5 & 0.5 & 0.5 & 0 & 0 & 0 \\
\hline & & 5 & Cylindrical grip & Chips can & 1 & 1 & 1 & 0 & 0 & 0 \\
\hline & & 6 & Diagonal volar grip & $\begin{array}{l}\text { Phillips } \\
\text { screwdriver }\end{array}$ & 0.5 & 0.5 & 0.5 & 1 & 1 & 1 \\
\hline & & 7 & Lateral pinch & Bowl & 1 & 1 & 1 & 0 & 0 & 0 \\
\hline & & 8 & Pulp pinch & Small marker & 0 & 0.5 & 0 & 0 & 1 & 0 \\
\hline & & 9 & $\begin{array}{l}\text { Index } \\
\text { pointing/pressing }\end{array}$ & Timer & 1 & 1 & 1 & 1 & 1 & 1 \\
\hline & & 10 & Hook & Pitcher base & 1 & 1 & 1 & 1 & 0.5 & 0.5 \\
\hline & & 11 & Spherical grip & Softball & 1 & 0.5 & 1 & 1 & 0 & 1 \\
\hline & & 12 & Tripod pinch & Tuna can & 1 & 1 & 1 & 0 & 0 & 0 \\
\hline & & 13 & Extension grip & Cracker box & 0 & 0 & 0 & 0 & 0 & 0 \\
\hline & & 14 & Cylindrical grip & Coffee can & 0.5 & 0.5 & 0.5 & 0 & 0 & 0 \\
\hline & & 15 & Diagonal volar grip & Spatula & 0.5 & 0.5 & 0.5 & 1 & 1 & 1 \\
\hline & & 16 & Lateral pinch & XS Clamp & 0 & 0.5 & 0.5 & 0 & 1 & 0.5 \\
\hline & & 17 & Pulp pinch & Plastic pear & 0 & 0.5 & 0.5 & 0 & 1 & 1 \\
\hline & & 18 & Platform & Plate & 1 & 1 & 1 & - & - & - \\
\hline & & 19 & Hook & $\begin{array}{l}\text { Wood blocks } \\
\text { with rope }\end{array}$ & 1 & 1 & 1 & 0.5 & 0.5 & 0.5 \\
\hline & & 20 & Spherical grip & $\begin{array}{l}\text { Mini soccer } \\
\text { ball }\end{array}$ & 0.5 & 0.5 & 0.5 & 0 & 0 & 0 \\
\hline & & 21 & Tripod pinch & Golf ball & 1 & 1 & 1 & 1 & 0.5 & 1 \\
\hline & & 22 & Extension grip & Pudding box & 0.5 & 0.5 & 0.5 & 0 & 0 & 0 \\
\hline & & 23 & Cylindrical grip & Power drill & 0.5 & 0.5 & 0.5 & 0 & 0 & 0 \\
\hline & & 24 & Diagonal volar grip & Skillet & 0 & 0 & 0 & 0 & 0 & 0 \\
\hline & & 25 & Lateral pinch & Key & 1 & 1 & 0 & 0 & 0 & 0 \\
\hline & & 26 & Pulp pinch & Washer 10mm & 0.5 & 0.5 & 0.5 & 1 & 1 & 1 \\
\hline \multirow{7}{*}{ InMoov } & \multirow{7}{*}{1} & 1 & Hook & Skillet lid & 1 & 1 & 1 & 0.5 & 0.5 & 0.5 \\
\hline & & 2 & Spherical grip & Plastic apple & 0.5 & 0.5 & 0.5 & 0.5 & 1 & 1 \\
\hline & & 3 & Tripod pinch & Large marker & 1 & 1 & 1 & 1 & 1 & 1 \\
\hline & & 4 & Extension grip & Plate & 0 & 0 & 0 & 0 & 0 & 0 \\
\hline & & 5 & Cylindrical grip & Chips can & 0.5 & 0.5 & 0.5 & 0 & 0 & 0 \\
\hline & & 6 & Diagonal volar grip & $\begin{array}{l}\text { Phillips } \\
\text { screwdriver }\end{array}$ & 0.5 & 0.5 & 0.5 & 1 & 1 & 1 \\
\hline & & 7 & Lateral pinch & Bowl & 1 & 1 & 1 & 0 & 0 & 0 \\
\hline
\end{tabular}




\begin{tabular}{|c|c|c|c|c|c|c|c|c|c|c|}
\hline & & 8 & Pulp pinch & Small marker & 0 & 0 & 0 & 0 & 0 & 0 \\
\hline & & 9 & $\begin{array}{l}\text { Index } \\
\text { pointing/pressing }\end{array}$ & Timer & 1 & 1 & 1 & 1 & 1 & 1 \\
\hline & & 10 & Hook & Pitcher base & 1 & 1 & 1 & 1 & 0.5 & 1 \\
\hline & & 11 & Spherical grip & Softball & 0.5 & 0.5 & 0.5 & 0 & 0 & 0 \\
\hline & & 12 & Tripod pinch & Tuna can & 1 & 1 & 1 & 0 & 0 & 0 \\
\hline & & 13 & Extension grip & Cracker box & 0.5 & 0 & 0 & 0 & 0 & 0 \\
\hline & & 14 & Cylindrical grip & Coffee can & 0.5 & 0.5 & 0.5 & 0 & 0 & 0 \\
\hline & & 15 & Diagonal volar grip & Spatula & 0.5 & 0.5 & 0.5 & 1 & 1 & 1 \\
\hline & & 16 & Lateral pinch & XS Clamp & 1 & 1 & 0 & 1 & 1 & 0 \\
\hline & & 17 & Pulp pinch & Plastic pear & 0 & 0.5 & 0 & 0 & 0 & 0 \\
\hline & & 18 & Platform & Plate & 1 & 1 & 1 & - & - & - \\
\hline & & 19 & Hook & $\begin{array}{l}\text { Wood blocks } \\
\text { with rope }\end{array}$ & 1 & 1 & 1 & 0.5 & 0.5 & 0.5 \\
\hline & & 20 & Spherical grip & $\begin{array}{l}\text { Mini soccer } \\
\text { ball }\end{array}$ & 0.5 & 0.5 & 0.5 & 0 & 0 & 0 \\
\hline & & 21 & Tripod pinch & Golf ball & 0.5 & 0.5 & 0.5 & 1 & 1 & 1 \\
\hline & & 22 & Extension grip & Pudding box & 0.5 & 0.5 & 0.5 & 0 & 0 & 0 \\
\hline & & 23 & Cylindrical grip & Power drill & 0.5 & 0.5 & 0.5 & 0 & 0 & 0 \\
\hline & & 24 & Diagonal volar grip & Skillet & 0 & 0 & 0 & 0 & 0 & 0 \\
\hline & & 25 & Lateral pinch & Key & 0.5 & 0.5 & 0.5 & 1 & 0.5 & 1 \\
\hline & & 26 & Pulp pinch & Washer 10mm & 0.5 & 0 & 0.5 & 1 & 0 & 1 \\
\hline \multirow{24}{*}{ InMoov } & \multirow{24}{*}{2} & 1 & Hook & Skillet lid & 1 & 1 & 1 & 0.5 & 0.5 & 0.5 \\
\hline & & 2 & Spherical grip & Plastic apple & 0.5 & 0.5 & 0.5 & 1 & 1 & 1 \\
\hline & & 3 & Tripod pinch & Large marker & 0.5 & 0.5 & 0.5 & 1 & 1 & 1 \\
\hline & & 4 & Extension grip & Plate & 0.5 & 0 & 0 & 0 & 0 & 0 \\
\hline & & 5 & Cylindrical grip & Chips can & 0.5 & 0 & 0.5 & 0 & 0 & 0 \\
\hline & & 6 & Diagonal volar grip & $\begin{array}{l}\text { Phillips } \\
\text { screwdriver }\end{array}$ & 0.5 & 0.5 & 0.5 & 1 & 1 & 1 \\
\hline & & 7 & Lateral pinch & Bowl & 1 & 1 & 1 & 0 & 0 & 0 \\
\hline & & 8 & Pulp pinch & Small marker & 0 & 0 & 0 & 0 & 0 & 0 \\
\hline & & 9 & $\begin{array}{l}\text { Index } \\
\text { pointing/pressing }\end{array}$ & Timer & 1 & 1 & 1 & 1 & 1 & 1 \\
\hline & & 10 & Hook & Pitcher base & 1 & 1 & 1 & 0.5 & 1 & 1 \\
\hline & & 11 & Spherical grip & Softball & 0.5 & 0.5 & 0.5 & 0 & 0 & 0 \\
\hline & & 12 & Tripod pinch & Tuna can & 1 & 1 & 1 & 0.5 & 0.5 & 0.5 \\
\hline & & 13 & Extension grip & Cracker box & 0.5 & 0.5 & 0 & 0 & 0 & 0 \\
\hline & & 14 & Cylindrical grip & Coffee can & 0 & 0.5 & 0 & 0 & 0 & 0 \\
\hline & & 15 & Diagonal volar grip & Spatula & 0.5 & 0.5 & 0.5 & 1 & 1 & 1 \\
\hline & & 16 & Lateral pinch & XS Clamp & 0.5 & 1 & 0 & 1 & 0 & 0 \\
\hline & & 17 & Pulp pinch & Plastic pear & 0 & 0 & 0 & 0 & 0 & 0 \\
\hline & & 18 & Platform & Plate & 1 & 1 & 1 & - & - & - \\
\hline & & 19 & Hook & $\begin{array}{l}\text { Wood blocks } \\
\text { with rope }\end{array}$ & 1 & 1 & 1 & 0.5 & 0.5 & 0.5 \\
\hline & & 20 & Spherical grip & $\begin{array}{l}\text { Mini soccer } \\
\text { ball }\end{array}$ & 0.5 & 0.5 & 0.5 & 0 & 0 & 0 \\
\hline & & 21 & Tripod pinch & Golf ball & 1 & 1 & 0 & 1 & 1 & 0 \\
\hline & & 22 & Extension grip & Pudding box & 0.5 & 0.5 & 0.5 & 0 & 0 & 0 \\
\hline & & 23 & Cylindrical grip & Power drill & 0.5 & 0.5 & 0.5 & 0 & 0 & 0 \\
\hline & & 24 & Diagonal volar grip & Skillet & 0 & 0 & 0 & 0 & 0 & 0 \\
\hline
\end{tabular}




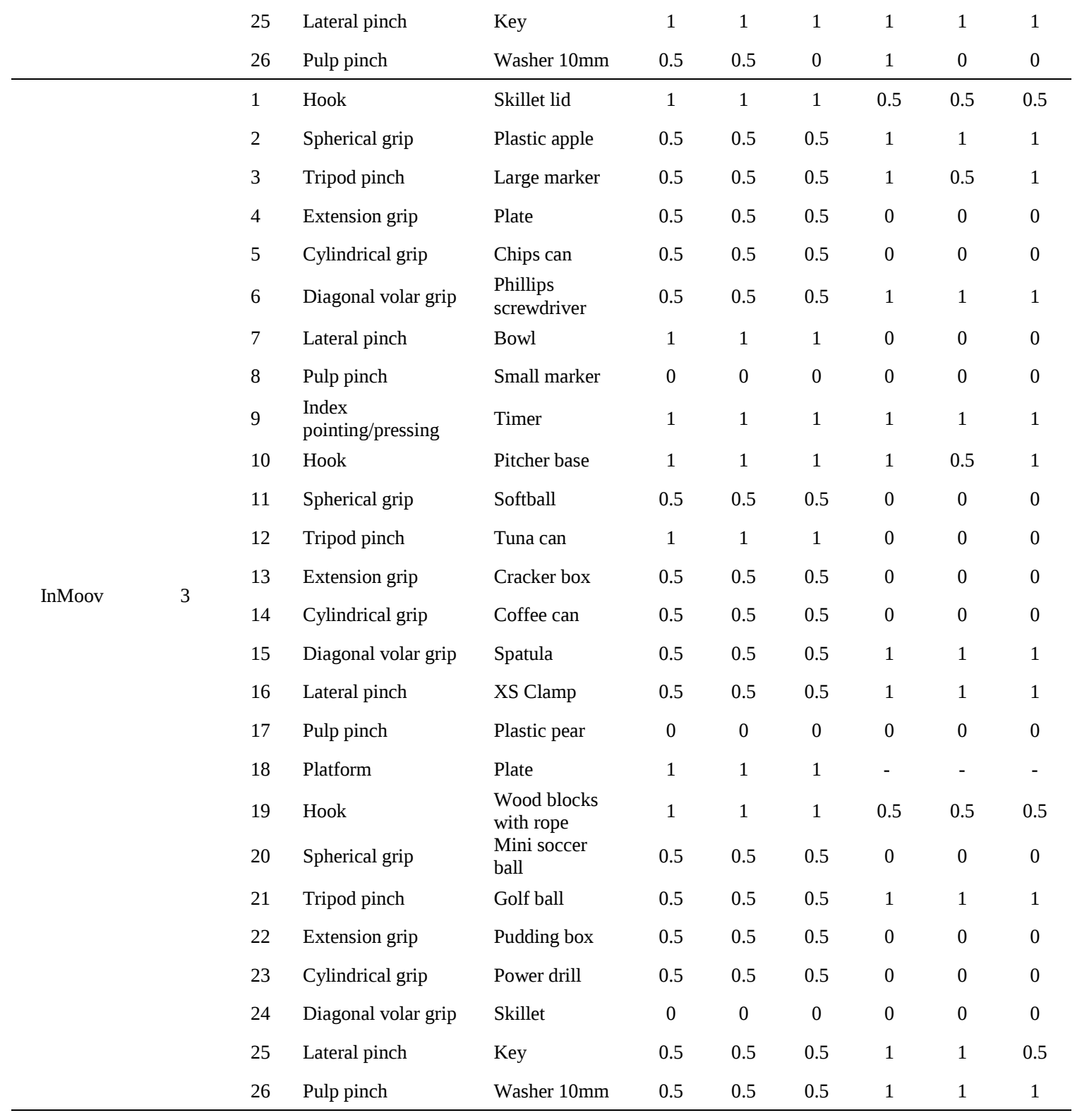




\section{Chapter 4}

Anthropomorphism Index of
Mobility for Artificial Hands 

This chapter corresponds exactly to the published paper:

Anthropomorphism Index of Mobility for Artificial Hands Immaculada Llop-Harillo, Antonio Pérez-González, and Verónica Gracia-Ibáñez

Applied Bionics and Biomechanics, 2019, 1-11, 7169034

\begin{abstract}
The increasing development of anthropomorphic artificial hands makes necessary quick metrics that analyze their anthropomorphism. In this study, a human grasp experiment on the most important grasp types was undertaken in order to obtain an Anthropomorphism Index of Mobility (AIM) for artificial hands. The AIM evaluates the topology of the whole hand, joints and degrees of freedom (DoFs), and the possibility to control these DoFs independently. It uses a set of weighting factors, obtained from analysis of human grasping, depending on the relevance of the different groups of DoFs of the hand. The computation of the index is straightforward, making it a useful tool for analyzing new artificial hands in early stages of the design process and for grading human-likeness of existing artificial hands. Thirteen artificial hands, both prosthetic and robotic, were evaluated and compared using the AIM, highlighting the reasons behind their differences. The AIM was also compared with other indexes in the literature with more cumbersome computation, ranking equally different artificial hands. As the index was primarily proposed for prosthetic hands, normally used as nondominant hands in unilateral amputees, the grasp types selected for the human grasp experiment were the most relevant for the human nondominant hand to reinforce bimanual grasping in activities of daily living. However, it was shown that the effect of using the grasping information from the dominant hand is small, indicating that the index is also valid for evaluating the artificial hand as dominant and so being valid for bilateral amputees or robotic hands.
\end{abstract}

\title{
Keywords
}

Anthropomorphism, artificial hand, index of function, metric, principal component analysis, prosthetic hand 


\section{$144 \mid$ Chapter 4}

\section{Introduction}

In recent years, it has been an increasing development of new affordable and anthropomorphic prosthetic hands (Belter et al., 2013; ten Kate et al., 2017) as a consequence of the improvements in 3D-printing technologies. The human hand is a complex and marvelous tool whose dexterity has not been achieved by any artificial hand. Evaluating the functional similarity of artificial hands with the human hand is essential for improving current anthropomorphic hand designs. Assessing the capability of the prostheses to perform the main grasp types (GTs) of human grasping could give an insight into the level of functionality restored in patients. Metrics or indexes that quantify numerically the level of anthropomorphism are the way to grade human-likeness and to provide specifications for maximizing the anthropomorphic functionality while designing new artificial hands.

Belter et al. (Belter et al., 2013) reviewed and compared the mechanical properties of different prosthetic hands, as their degrees of freedom (DoFs), range of motion, and weight and number of actuators, but an index to compare those properties with the human hand was not defined. Some other previous studies tried to quantify the anthropomorphism of artificial hands with a numerical index. Feix et al. (Feix et al., 2013) proposed a metric for comparing the anthropomorphic motion capability of robotic and prosthetic hands, the anthropomorphism index (AI), being its computation cumbersome and based only on the position and orientation of the distal phalanges in different GTs. Liarokapis et al. (Liarokapis et al., 2013) defined an anthropomorphism index to assess the robot's ability to mimic the human hand based on the comparison of the finger phalanx workspaces and also the workspaces of the fingers' base frames. Liu et al. (Liu et al., 2015) proposed twelve quantified prosthetic hand anthropomorphism evaluation indexes including physical and actuation properties, among which is included a DoF configuration evaluation. This index was based on a matrix of DoF configuration where the element of the matrix is set to 1 if there exist an artificial DoF in the corresponding position, otherwise is set to 0. However, this approach does not take into account the relevance of each DoF for grasping during activities of daily living (ADL) nor the underactuation in the joints. Underactuation in artificial hands (Birglen et al., 2008) allows to use less actuators than DoFs while keeping versatility to adapt GTs to different object shapes.

Prostheses design could be different depending on its use for a dominant or nondominant hand; however, in the case of a patient who still has a healthy 
hand, the most appropriate strategy would be to consider the remaining hand as dominant (Cornwall, 2007; Yancosek and Mullineaux, 2011). Thereby, the design of the prosthesis should be focused for a nondominant hand reinforcing bimanual grasping. The importance of the different GTs for personal autonomy of the patients in ADL has been studied previously by the authors (Gracia-Ibáñez et al., 2018), being pulp pinch (PP) (26\%), extension grip (EG) (20.8\%), tripod pinch (TP) (10.4\%), and transverse volar grip (TVG) (8.7\%), the most relevant GTs for a nondominant hand to reinforce bimanual grasping, representing together with the nonprehensile one, almost $90 \%$ of relevance for autonomy.

In the previous studies by the authors (Gracia Ibañez, 2016), the posture of the right hand from 24 healthy subjects performing 24 representative ADL was recorded with an instrumented glove. ADL were selected from the WHO's International Classification of Functioning, Disability and Health (World Health Organization, 2002). By applying principal component analysis (PCA), five factors explaining $73.7 \%$ of the variance were obtained. As shown in Figure 1, the five main principal components (PCs) of the DoFs of the human hand in ADL were "PC1: digit arching" (flexion of the interphalangeal joints), "PC2: closure" (combination of abduction of the fingers, except for the thumb, with flexion of the metacarpophalangeal joints), "PC3: palmar arching”, "PC4: lateral pinch" (represents the lateral opposition of the thumb to the index), and "PC5: opposition" (represents the pad-to-pad opposition of the thumb to the little finger).

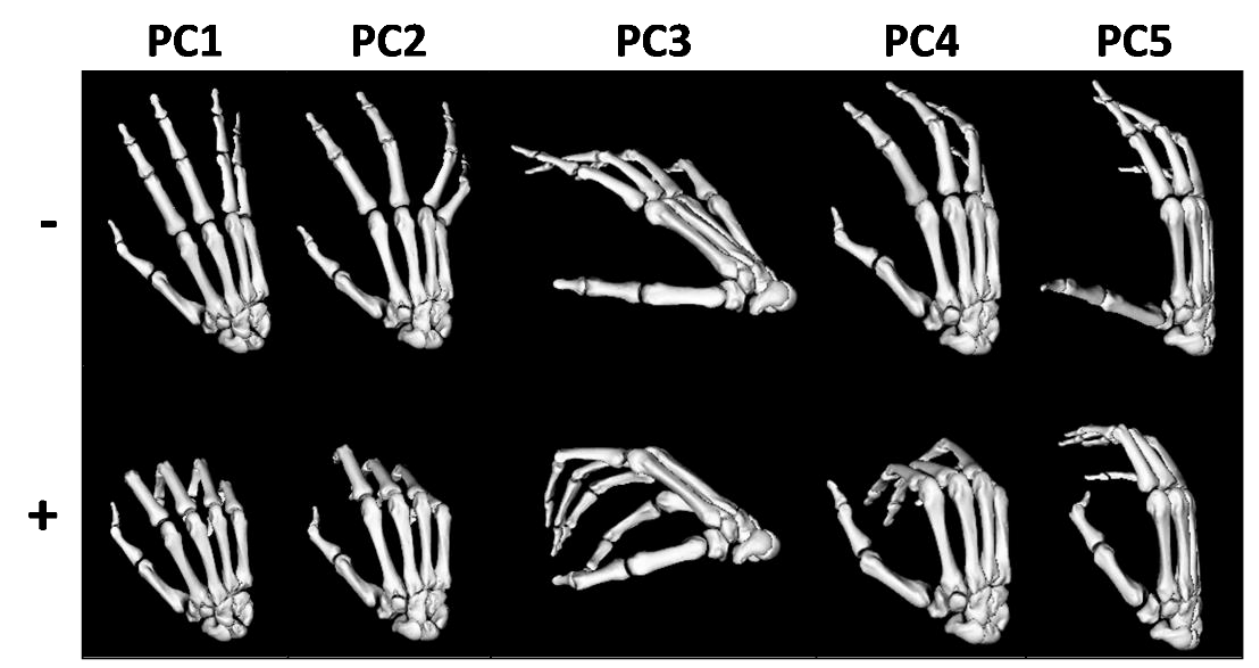

Figure 1. Principal components of the degrees of freedom of the human hand performing activities of daily living obtained in (Gracia Ibañez, 2016) (PC1: digit arching, PC2: closure, PC3: palmar arching, PC4: lateral pinch, PC5: opposition). 


\section{6 | Chapter 4}

The aim of this study is to propose an index to measure the anthropomorphism of prosthetic hands, based on the comparison of the topology of the whole hand (joints and DoFs) and on the possibility to control these DoFs independently. The computation of the index, referred to as Anthropomorphism Index of Mobility (AIM), should weight each DoF depending on its importance for grasping in ADL. To define this importance, we used the information from previous experimental tests performed in the group and specific tests developed in this study on the main GTs. Furthermore, a preliminary study (Llop-Harillo et al., 2017) carried out on four human healthy subjects encouraged us to go deep in the study by increasing the number of subjects, improving the definition of the index, and widening the analysis of its validity to the different types of artificial hands. The AIM is intended to be a quick computation index based on the biomechanics of the human hand and thus providing a way to compare their functional anthropomorphism. Moreover, the relevance of each DoF for functionality, obtained by tests on the human hand in this study, is intended to be useful for other applications in artificial hand design.

\section{Materials and Methods}

\subsection{Human Grasp Experiment}

With the purpose of taking into account in the AIM the relevance of each DoF according to its importance for functional grasping, an experiment to measure the kinematics of the human hand in functional grasps was carried out. Twenty subjects, ten males and ten females, all of whom were righthanded and free of hand pathologies or injuries, performed the most relevant GTs for a nondominant hand to reinforce bimanual grasping in ADL (PP, EG, TP, and TVG (Gracia-Ibáñez et al., 2018)). Although the grasps were selected for a nondominant hand (most common use of a hand prosthesis for unilateral amputees), subjects were asked to perform grasps with their dominant hand to get the most natural performance of human grasping. The study was approved by the Ethics Committee of the University, and all the subjects gave their written informed consent. The ages of the subjects ranged intentionally between 20 and 51 , being the average $35 \pm 8$, in order to prevent kinematic alterations due to joint degeneration from ageing. Subjects were selected so that the distribution of hand sizes was representative of the population (Vergara et al., 2018). The hand width ranged from 70 to $96 \mathrm{~mm}$ with an average of $83 \mathrm{~mm}$, and the hand length ranged from 170 to $210 \mathrm{~mm}$ with an average of $185 \mathrm{~mm}$. 
Twelve objects of different sizes were selected from the Yale-CMUBerkeley Object and Model Set (Calli et al., 2015), three for each of the four GTs (PP, EG, TP, and TVG), in order to cover most common requirements in ADL for each one (Figure 2). The subjects were sitting with the hands in the table in a comfortable way: the arms close to the body and parallel to the sagittal plane, the elbows flexed $90^{\circ}$, the wrist on the edge of the table, and the hands laying on the table palms down in a natural posture. This was the starting and ending posture for each grasping action. Subjects were instructed on the different GTs to perform with each object, and objects to be grasped were situated one by one by the researcher at a distance of $30 \mathrm{~cm}$ in front of the subjects. Subjects were free to practice the grasps to be sure that it is in the correct posture before starting the recordings. The steps to perform the grasps during the experiment consisted of the following: grasping the object from the table with the correct hand posture/GT, lift it up during two seconds, and finally, release the object again on the table and return the hand to the starting position. The sequence of the twelve objects to grasp during the experiment is shown in Figure 2 in the specified order. The experiment was repeated three times per subject.

The kinematics of the hand while performing the grasping postures was recorded $(100 \mathrm{~Hz})$ using an instrumented right hand glove with 18 sensors (CyberGlove Systems LLC; San Jose, CA). DoF kinematics corresponding to 16 joint angles (marked with an asterisk in Table 1) was obtained using a previously validated protocol (Gracia-Ibáñez et al., 2017a): metacarpophalangeal flexion (MCP1 to MCP5, 1 to 5 meaning thumb to little digits), interphalangeal flexion of the thumb (IP1), proximal interphalangeal flexion of the fingers (PIP2 to PIP5), flexion and abduction of the carpometacarpal joint of the thumb (CMC1), relative abduction between finger MCPs (index-middle, middle-ring, and ring-little), and palmar arching. Prior to the tests with objects, the CyberGlove was calibrated for each subject following the calibration procedure (Gracia-Ibáñez et al., 2017a). Starting and final positions while the hand is not moving were trimmed from the recordings. Then, they were filtered with a 2nd-order 2way low-pass Butterworth filter with cut-off frequency of $5 \mathrm{~Hz}$ (GraciaIbáñez et al., 2017b; Patel et al., 2017). The tests were video recorded. 

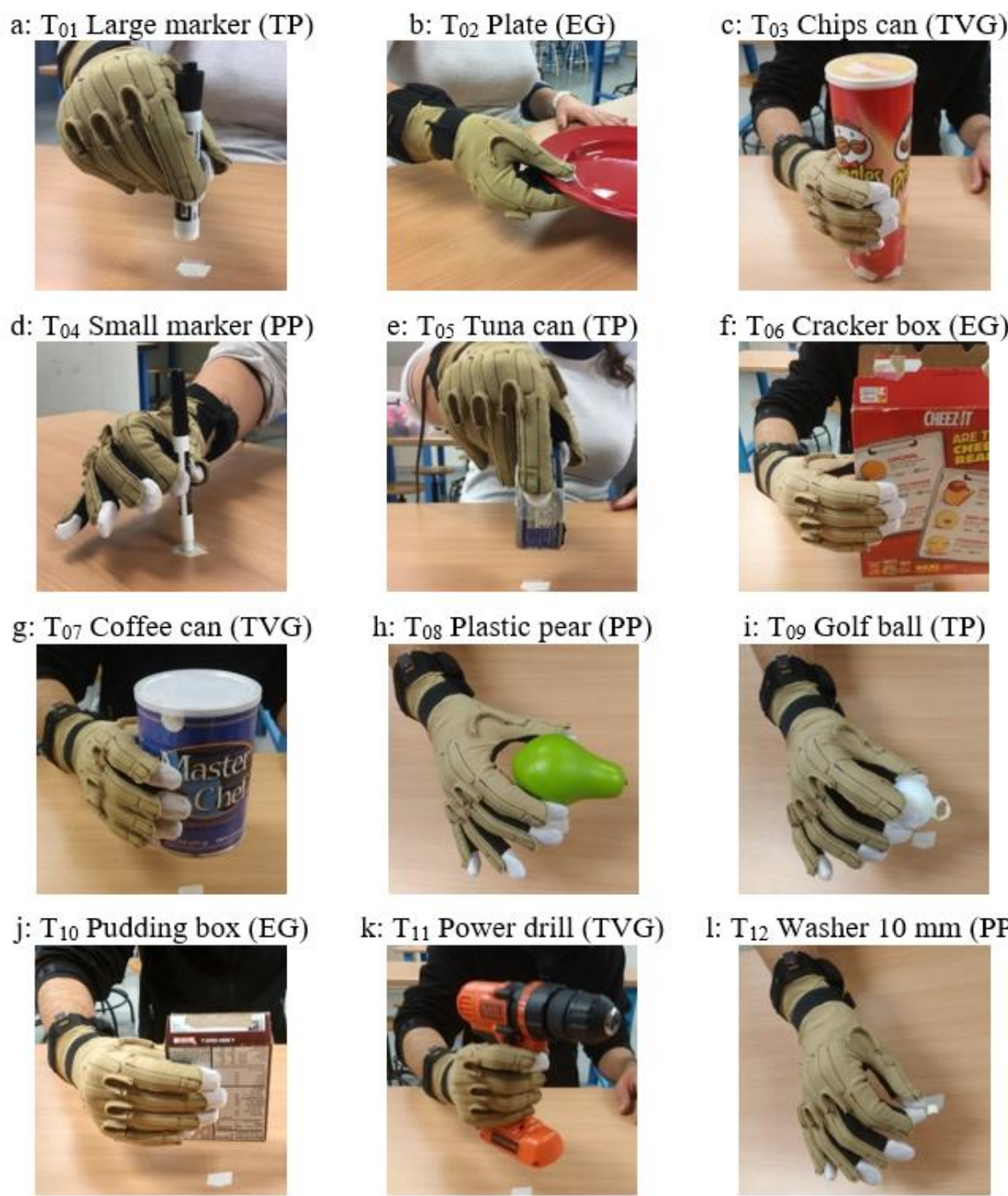

$\mathrm{k}: \mathrm{T}_{11}$ Power drill (TVG)

1: $\mathrm{T}_{12}$ Washer $10 \mathrm{~mm}(\mathrm{PP})$
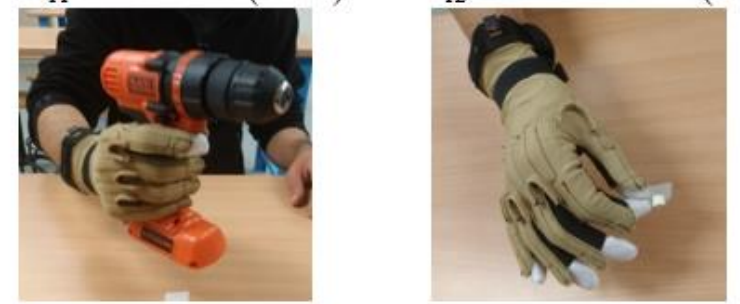

Figure 2. Grasping tasks of the experiment (a-l). $T_{g}$ are the tasks ordered (g: indicates the order) followed by the object of the Yale-CMU-Berkeley Object and Model Set (Calli et al., 2015) to grasp and in brackets the grasp type to be performed in each task (TP: tripod pinch, EG: extension grip, TVG: transverse volar grip, PP: pulp pinch).

\subsection{Index Definition}

The Anthropomorphism Index of Mobility (AIM) for an artificial hand was defined based on two main factors: (1) the DoFs present in the hand along with its method of actuation and (2) the relevance of these DoFs for grasping in ADL.

The DoFs of the human hand (HH) (Kapandji, 1982; Sancho-Bru, 2000) were classified into four different functional groups for defining the AIM (Table 1): finger flexion-extension (12 in $\mathrm{HH}$ ), finger abduction-adduction (4 in $\mathrm{HH}$ ), palmar arching (2 in $\mathrm{HH}$ ), and thumb opposition (5 in $\mathrm{HH}$ ). 
Table 1. Joints and degrees of freedom (DoFs) of the human hand corresponding to the four different groups of DoFs defined. (1: thumb, 2: index finger, 3: middle finger, 4: ring finger, 5: little finger; CMC: carpometacarpal joint, MCP: metacarpophalangeal joint, PIP: proximal interphalangeal joint, DIP: distal interphalangeal joint, IP: interphalangeal joint; *16 joint angles measured during the experiment with the CyberGlove).

\begin{tabular}{ll}
\hline Groups of DoFs & Joints and DoFs of the human hand \\
\hline Finger flexion-extension & MCP2_Flexion* \\
(F/E) & PIP2_Flexion* \\
& DIP2_Flexion \\
& MCP3_Flexion* \\
& PIP3_Flexion* \\
& DIP3_Flexion \\
& MCP4_Flexion* \\
& PIP4_Flexion* \\
& DIP4_Flexion \\
& MCP5_Flexion* \\
& PIP5_Flexion* \\
& DIP5_Flexion \\
\hline Finger abduction-adduction & MCP2_Abduction* \\
(AB/AD) & MCP3_Abduction \\
& MCP4_Abduction* \\
\hline Palmar arching & MCP5_Abduction* \\
(P.ARC) & CMC5_Flexion* \\
\hline Thumb opposition & CMC4_Flexion \\
(T.OPP) & CMC1_Flexion* \\
& CMC1_Abduction* \\
& MCP1_Flexion* \\
& MCP1_Abduction \\
\hline
\end{tabular}

The Anthropomorphism Index of Mobility (AIM) was defined with Equation 1:

$$
A I M=\sum_{i}\left(k_{i} \cdot w_{i}\right)
$$

where the summation extends for $i=1,2,3,4$, corresponding to each one of the four groups of DoFs (Table 1: F/E, AB/AD, P.ARC, and T.OPP), the factor $k_{i}$ accounts for the type of actuation of the DoFs included in this group, and the factor $w_{i}$ is a weighting coefficient depending on the relevance of this group of DoF for grasping in ADL. Both the term $k_{i}$ and the weighting factor $w_{i}$ were defined to have a range between 0 and 1 , and the sum of weighting coefficients $w_{i}$ for the four groups is unity, so that the AIM reach a maximum value of 1 for the human hand and a very low value for an artificial hand with very poor anthropomorphism. 
The factor $k_{i}$ for each group $i$ was defined to get a high value if the method of actuation for the DoFs in that group allows to control them independently, as in the human hand, and a lower value if the motions of these DoFs are highly coupled during motion. To this end, each DoF in the evaluated hand was classified according to the types included in Table 2.

Table 2. Classification of the DoF depending on the type of actuation and numeric coefficient associated.

\begin{tabular}{|c|c|c|}
\hline Class & Type of actuation of the DoF & $C$ \\
\hline$\overline{\mathrm{A}}$ & DoF actuated by one independent motor or actuator & 1 \\
\hline B & $\begin{array}{l}\text { DoF underactuated with other DoFs without a rigid coupling, allowing } \\
\text { adaptive grasps (tendons, elastic elements) }\end{array}$ & 0.75 \\
\hline $\mathrm{C}$ & $\begin{array}{l}\text { DoF underactuated with other DoFs with a rigid coupling, not allowing } \\
\text { adaptive grasp (linkages) }\end{array}$ & 0.5 \\
\hline $\mathrm{D}$ & No actuation on the DoF, but passive motion allowed & 0.25 \\
\hline $\mathrm{E}$ & DoF absent in the artificial hand & 0 \\
\hline
\end{tabular}

The independent mobility of a DoF can be ranked qualitatively from better to worse, depending on its class, as A $>$ B $>$ C $>$ D $>$ E. Note that B class was considered better than $\mathrm{C}$ because it allows mechanical adaptation of the finger to the shape of the object to be grasped and do not suffer from mechanical singular configurations (Birglen et al., 2008). Pugh's method used in concept design evaluation (Ullman, 2010) was employed to convert the ranked list of methods of actuation of the DoFs into a list of numerical coefficients $c$ (last column in Table 2). However, the independent mobility of a DoF is associated not only with the type of actuation in this particular DoF but also with that of the DoFs more proximal in the same serial chain of a digit, i.e., for a finger, the mobility for flexion in the PIP joint is dependent on the mobility for flexion in the MCP joint. Consequently, for that case, the coefficient $c_{i j}$ for the DoF $j$ of the group $i$ was obtained as the multiplication of the coefficient $c$ of this DoF and those located proximally in the same serial kinematic chain. In addition, for assigning the coefficient $c$ to several DoFs underactuated by the same motor or actuator, class A was considered for only one of them and class $\mathrm{B}$ or $\mathrm{C}$ for others. If a motor actuates several DoFs included in different groups $i$, the coefficient 1 corresponding to class A was divided among the number of groups and this fraction was assigned to only one of the DoFs in this group, being others classified as either B or C. Finally, the factor $k_{i}$ for each group $i$ was defined with Equation 2, by summing the terms $c_{i j}$ in the group $i$ and dividing by the number of DoFs of the human hand in this group $\left(n_{i}\right)$, which is, according to Table 1,12 for $i=1,4$ for $i=2,2$ for $i=3$, and 5 for $i=4$. 


$$
k_{i}=\frac{\sum_{j} c_{i j}}{n_{i}}
$$

The weighting factor $w_{i}$ in Equation 1, accounting for the relative relevance of the DoFs of the group $i$ for grasping in ADL, was defined with Equation 3.

$$
w_{i}=\sum_{k}\left(r_{i k} \cdot s_{k}\right)
$$

In Equation 3, $r_{i k}$ weights the relative contribution of the group of DoFs $i \quad(i=1,2,3,4)$ in human hand functionality represented through $\mathrm{PC}_{k}$ $(k=1,2,3,4,5)$, corresponding to each of the five kinematic functional synergies (see Figure 1) found in a previous study (Gracia Ibañez, 2016). These PCs account for $73.7 \%$ of the variance when performing a wide set of representative ADL. The loading matrix of the PCs obtained in that study, which can be found in Supplementary Materials, was used to calculate $r_{i k}$ as shown in Equations 4 and 5. For a $\mathrm{PC}_{\mathrm{k}}, r_{i k}$ was computed as the sum of absolute values of the loadings $l_{i j k}$ for the DoFs $j$ included in the group $i$ (according to Table 1) divided by the sum of the absolute value of all the loadings of that $\mathrm{PC}_{k}$.

$$
\begin{aligned}
& r_{i k}=\frac{\sum_{j}\left|l_{i j k}\right|}{a_{k}} \\
& a_{k}=\sum_{i} \sum_{j}\left|l_{i j k}\right|
\end{aligned}
$$

On the other hand, $s_{k}$ in Equation 3 contains the information about the importance of the $\mathrm{PC}_{k}$ in the most relevant GTs. To compute this term, first, the human hand kinematics was obtained from the human grasp experiment explained above, but to consider the relation with the functionality of the human hand during ADL, kinematics was transformed to be expressed as scores $f_{t k}$ referred to the five functional PCs (Figure 1) instead of being expressed in the original sixteen variables (joint angles). This information can be found in Supplementary Materials. A greater absolute value of the score of a $\mathrm{PC}_{\mathrm{k}}$ in one particular instant $t$ indicates that the position of the hand is better represented by this $\mathrm{PC}_{\mathrm{k}}$. Next, for each of the twelve grasping tasks $g$ (Figure 2), the absolute value of the scores $f_{t k}$ for each $\mathrm{PC}_{\mathrm{k}}$ was averaged during the task (Equation 6) and then (Equation 7) these means $v_{g b p}$ were averaged across subjects $b$ and repetitions $p$. The resulting means $v_{g}$ were normalized (Equation 8) with respect to their sum across PCs $h_{g}$ (Equation 9), providing the relative contribution of the five PCs to each grasping task $n_{g}$. Finally, these relative contributions were weight-averaged 


\section{2 | Chapter 4}

by the relative relevance of the GT for autonomy of each grasping task $z_{g}$ and divided by 3 because three objects were considered for each GT (Equation 10). The weight $z_{g}$ for averaging was obtained from the relative use of the four main GT for a nondominant hand in bimanual grasping (Gracia-Ibáñez et al., 2018): 39.5\% for PP, 31.6\% for EG, 15.8\% for TP, and $13.2 \%$ for TVG.

$$
\begin{aligned}
& \left(v_{g b p}\right)_{k}=\frac{\sum_{t=1}^{m}\left|f_{t k}\right|}{m} \\
& \left(v_{g}\right)_{k}=\frac{\sum_{b} \sum_{p}\left(v_{g b p}\right)_{k}}{b \cdot p} \\
& \left(n_{g}\right)_{k}=\frac{\left(v_{g}\right)_{k}}{h_{g}} \\
& h_{g}=\sum_{k}\left(v_{g}\right)_{k} \\
& s_{k}=\frac{\sum_{g}\left[\left(n_{g}\right)_{k} \cdot z_{g}\right]}{3}
\end{aligned}
$$

\subsection{Artificial Hands}

With the objective of exemplifying the use of the AIM and verifying its utility, it was computed for several artificial hands with different topologies and actuation systems. The AIM was obtained for different affordable 3Dprinted prosthetic hands, including the IMMA hand designed by the authors (Llop-Harillo and Pérez-González, 2017), some advanced commercial prosthetic hands, and other artificial hands. Some hands of these two later groups have been evaluated with other indexes of anthropomorphism in the literature, such as the anthropomorphism index (AI) (Feix et al., 2013) and the Total Score of Anthropomorphism ( $\left.A_{R}\right)$ (Liarokapis et al., 2013). The main characteristics of the hands analyzed are described below.

\subsubsection{Affordable 3D-printed prosthetic hands}

i. IMMA Hand (Llop-Harillo and Pérez-González, 2017): 3D-printed five-digit prosthetic hand, with 6 DoFs actuated by tendons: flexion in each finger and flexion and abduction in the thumb. It has three phalanges per finger and its joints are elastic elements. This hand is just a prototype and cannot be used as a prosthesis directly, it needs a socket with motors and a control system, but after a study of the authors (Llop-Harillo et al., 2019) it is being considered here to be 
actuated by two motors following the two actuation synergies obtained from experiments with human actuation.

ii. Cyborg Beast (Zuniga et al., 2015): five-digit low-cost 3D-printed prosthetic hand for children with upper-limb differences. It is bodypowered using the wrist of the amputee as the unique actuator to drive all the finger tendons. It has two phalanges per finger and 5 DoFs: flexion in each finger and flexion of the thumb. Finger flexion is driven by tendons along the palmar surface of each finger. Elastic cords placed inside the dorsal aspect of the fingers provide passive finger extension. Its joints are Chicago screws and the materials used to print the different parts of the hand are PLA and ABS.

iii. Flexy-Hand (Gyrobot, 2014): 3D-printed five-digit prosthetic hand, with 5 DoFs actuated by tendons: flexion in each finger and flexion in the thumb. It has three phalanges per finger and two phalanges in the thumb. The retraction is made through flexible 3D-printed joints. It is body-powered using the wrist of the amputee as the unique actuator to drive all the finger tendons.

iv. KIT Prosthetic Hand (Weiner et al., 2018): five-digit 3D-printed hand prosthesis with underactuated mechanism, sensors, and embedded control system, developed by the Institute for Anthropomatics and Robotics (Karlsruhe Institute of Technology). Two motors (one for the four long fingers and other for the thumb) actuate $10 \mathrm{DoFs}$ (flexion of two joints in each finger) by means of tendons. The four long fingers are simultaneously driven via a force-distributing transmission based on the TUAT/Karlsruhe mechanism providing shape adaptivity (all fingers keep closing until contact regardless of blocked movement in other joints). The passive reopening of the fingers is obtained through custom made springs.

v. ADA (Open Bionics, 2018): Ada Hand V1.1 by Open Bionics is a five-digit myoelectric prosthetic hand entirely 3D-printed with flexible material. It is tendon driven and has two phalanges in each finger and one linear actuator for each digit driving their flexion.

\subsubsection{Commercial prosthetic hands}

i. i-Limb: myoelectronically controlled, externally powered, tendon linking, multiarticulating prosthetic hand of Touch Bionics (Touch Bionics, 2019) with eleven joints (two joints in each long finger and 


\section{4 | Chapter 4}

three in the thumb). It has five individually powered digits and powered thumb rotation, with manual override.

ii. Bebionic (Ottobock, 2017): multiarticulating myoelectric prosthetic hand developed by RSL Steeper with eleven joints (two joints in each long finger and three in the thumb). It has five actuators, one for each finger, and the thumb has two positions manually placed by the user with an inbuilt sensor detecting the position. Folding links allow the fingers to flex. Among the 14 different grip patterns and hand positions that it can achieve, one of them is the finger adduction grip.

iii. SensorHand Speed: the Ottobock SensorHand Speed (Ottobock, 2017) is a myoelectronically controlled hand with three actuated fingers, which are driven by the same motor. It is covered with a cosmetic glove that emulates the ring and little finger using a metal bar within the glove, which couples these fingers to the movements of the middle finger.

iv. Michelangelo Hand: five-digit technologically advanced prosthetic hand of Ottobock (Ottobock, 2017). Actively driven elements are the thumb, index finger, and middle finger while the ring finger and little finger passively follow the other fingers. The six joints are controlled by two actuators (one for the flexion/extension of the five digits and the second one allows the thumb to be electronically positioned in an additional axis of movement being abducted/adducted). The fingers are slightly abducted when the MCP joints are extended, and when flexed, the fingertips adduct and touch each other, providing a finger abduction/adduction mode.

\subsubsection{Other artificial hands}

i. FRH-4 Hand: this is a robot hand built for the mobile-assisting robot ARMAR (Gaiser et al., 2008). It has eight independent fluidic actuators: one in the metacarpus that allows the palm to flex in the middle, the index and middle fingers have two each, the ring and little fingers have one for both, and the thumb has two actuators.

ii. Barrett (Barrett Technology, 2013): three-digit programmable grasper of Robotnik. It has four brushless motors and three multijointed fingers (two phalanges connected by belt transmission), two of them have an extra DoF with $180^{\circ}$ of lateral mobility. 
iii. DLR/HIT II (Liu et al., 2008): used on Space Justin (humanoid upper body) for telemanipulation is a multisensory robot hand from Harbin Institute of Technology and DLR Institute for Robotics and Mechatronic. It has 15 DoFs, five identical modular fingers with two flat BLDC motors placed in the base. Each finger has three active DoFs (2 DoFs of flexion and one of abduction) and four joints (the motions of distal and medial phalanges are coupled by a linkage). The thumb is fixed in an appropriate orientation of the palm.

iv. Shadow (Shadow Robot Company, 2019): the Shadow Dexterous Hand is a humanoid robot hand created by the Shadow Robot Company. The four fingers have 2 one-axis joints (DIP and PIP) and one universal MCP joint; the little finger has an extra one-axis joint on the metacarpal to provide arching. The thumb has a one-axis joint (IP) and two universal joints (MCP and CMC). It contains 20 motors in the forearm (3 DoFs per finger, 5 DoFs in the thumb, 1 DoF in the palm, and 2 DoFs in the wrist).

\section{Results and Discussion}

Figure 3 shows a representative plot of the mean value and standard deviation across all the subjects (20) and repetitions (3) of the 16 joint angles measured with the CyberGlove while performing a grasp of one object of the human grasp experiment ( $\mathrm{T}_{02}$ of Figure 2 ).

Table 3 shows the relative contribution of the five PCs to each grasping task of the human grasp experiment $\left(n_{g}\right)_{k}$ (Equation 8) together with the final value of the parameter $s_{k}$ for each PC (Equation 10), obtained from these relative contributions weight-averaged by their importance in ADL $z_{g}$ (Gracia-Ibáñez et al., 2018).

The contributions of the different PCs to each grasping task ranged between $9.6 \%$ and 39.1\%, indicating that all the five PCs have a nonnegligible importance in the twelve grasping tasks analyzed. It can be seen that PC1, corresponding to "digit arching”, is predominant in grasping tasks involving PP (g=\{4,8,12\}). Moreover, "palmar arching” (PC3) and "lateral pinch" (PC4) synergies are less represented in average in the four main GTs considered in the experiment, leading to lower values of the final parameter $s_{k}$ for these PCs, although the difference with "opposition" (PC5) is small. "Digit arching" (PC1) is the most significant synergy, as indicated by the higher value of $s_{k}$. 


\section{6 | Chapter 4}

Table 4 shows the matrix $r_{i k}$ (Equation 4) containing the influence of the different groups of DoFs of the human hand on the five kinematic functional synergies (Figure 1) and the resulting parameter $w_{i}$ after applying Equation 3 with this matrix $r_{i k}$ and the vector $s_{k}$ (Table 3).
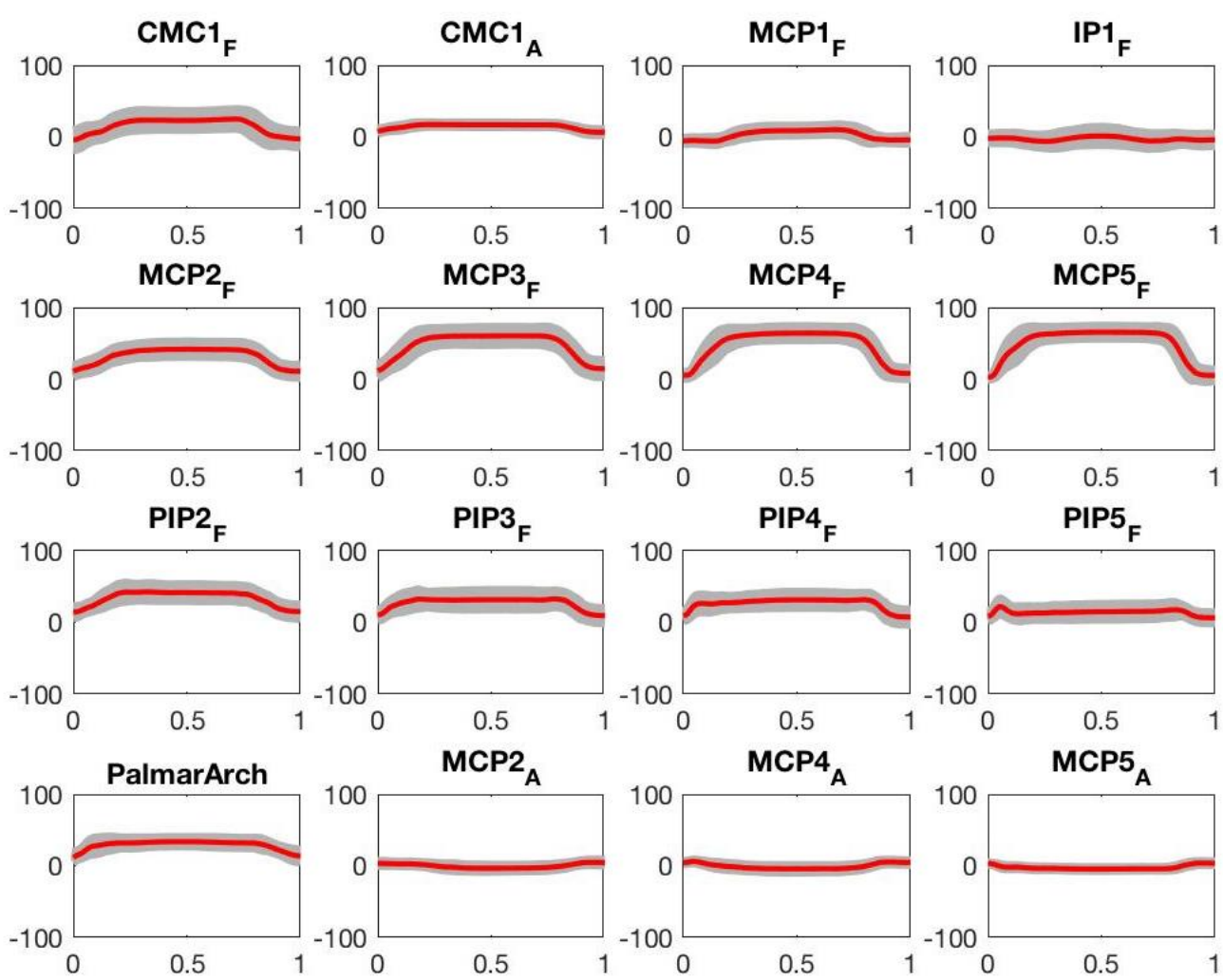

Figure 3. Mean and standard deviation of the 16 joint angles (in degrees) measured with the CyberGlove during the extension grip of the plate $\left(\mathrm{T}_{02}\right)$. The angles are normalized across time. (1: thumb, 2: index finger, 3: middle finger, 4: ring finger, 5: little finger; CMC: carpometacarpal joint, MCP:

metacarpophalangeal joint, PIP: proximal interphalangeal joint, DIP: distal interphalangeal joint, IP: interphalangeal joint; F: flexion/extension; A: abduction/adduction).

The parameter $w_{i}$ weights the relevance of the different groups of DoFs in the human grasps in ADL. These results indicate that finger flexionextension is by far the most relevant group of DoFs accounting for more than half of the functionality, followed by thumb opposition and finger abductionadduction. The palmar arching has a relevance of only 5\%. This result by itself is valuable for making decisions during the design of new artificial hand prototypes to maximize their functionality.

Table 5 shows the value of the parameter $k_{i}$ (Equation 2) for each group of DoFs for the artificial hands analyzed. The details about the computation for each hand $\left(c_{i j}\right)$ are supplied as Supplementary Materials. It can be seen 
that $\mathrm{F} / \mathrm{E}$ and T.OPP are the groups of DoFs mainly included and actively driven in the artificial hands, manifested by higher values of $k_{i}$. It is worth to note that this fact is coherent with the greater relevance of these groups of DoFs in ADL, as indicated by the parameter $w_{i}$ (Table 4). Notwithstanding, some hands as the SensorHand and Michelangelo showed low scores in F/E because of their rigid fingers without interphalangeal joints. The unique hand with the 5 DoFs in T.OPP actively driven is the Shadow hand. AB/AD is included actively in DLR/HIT II and Shadow hands and passively through the use of deformable joints in some 3D-printed hands. Finally, P.ARC is only present in FRH-4 and Shadow hands.

Table 3. Mean (SD) of the relative contribution $n_{g}$ of the five PCs in each grasping task $\mathrm{T}_{\mathrm{g}}$ (g: indicates the order of the tasks in Figure 2) and final value of the parameter $s_{k}$ for each PC.

\begin{tabular}{cccccc}
\hline & PC1 (\%) & PC2 (\%) & PC3(\%) & PC4 (\%) & PC5 (\%) \\
\hline $\mathrm{T}_{01}$ & $19.2(6.4)$ & $24.4(7.6)$ & $18.4(7.7)$ & $13.0(7.7)$ & $24.9(9.7)$ \\
$\mathrm{T}_{02}$ & $22.6(6.9)$ & $14.2(6.3)$ & $25.1(6.1)$ & $22.4(8.1)$ & $15.7(8.4)$ \\
$\mathrm{T}_{03}$ & $25.1(4.7)$ & $23.1(4.7)$ & $15.6(4.3)$ & $24.9(8.9)$ & $11.3(5.5)$ \\
$\mathrm{T}_{04}$ & $33.5(6.7)$ & $19.1(3.8)$ & $12.6(3.5)$ & $12.1(4.2)$ & $22.7(10.6)$ \\
$\mathrm{T}_{05}$ & $22.4(8.3)$ & $21.4(8.9)$ & $18.6(8.5)$ & $17.4(10.6)$ & $20.1(13.9)$ \\
$\mathrm{T}_{06}$ & $28.2(6.0)$ & $21.0(5.0)$ & $17.9(5.4)$ & $17.0(9.5)$ & $16.0(10.2)$ \\
$\mathrm{T}_{07}$ & $28.3(5.0)$ & $21.1(3.8)$ & $12.9(4.3)$ & $27.0(9.2)$ & $10.8(5.8)$ \\
$\mathrm{T}_{08}$ & $39.1(6.2)$ & $23.6(4.0)$ & $12.9(4.6)$ & $11.8(7.7)$ & $12.5(7.3)$ \\
$\mathrm{T}_{09}$ & $19.5(8.2)$ & $24.5(7.1)$ & $14.3(7.9)$ & $20.3(10.1)$ & $21.3(9.0)$ \\
$\mathrm{T}_{10}$ & $30.9(6.2)$ & $18.6(5.2)$ & $20.1(6.2)$ & $9.6(5.4)$ & $20.7(11.7)$ \\
$\mathrm{T}_{11}$ & $19.9(3.4)$ & $19.8(7.7)$ & $19.2(7.9)$ & $18.3(10.8)$ & $22.8(13.3)$ \\
$\mathrm{T}_{12}$ & $34.0(7.4)$ & $20.4(5.1)$ & $12.8(4.3)$ & $12.1(6.5)$ & $20.8(9.6)$ \\
\hline$S_{k}$ & 29.0 & 20.5 & 16.5 & 15.6 & 18.4 \\
\hline \multicolumn{5}{c}{}
\end{tabular}

Table 4. Matrix $r_{i k}$ and resulting $w_{i}$ (Equation 3).

\begin{tabular}{lccccc|c}
\hline Groups of DoFs & \multicolumn{5}{c|}{ Functional synergies } & \\
& PC1(\%) & PC2(\%) & PC3(\%) & PC4(\%) & PC5(\%) & $w_{i}(\%)$ \\
\hline Finger flexion-extension & 79.6 & 50.7 & 42.5 & 51.8 & 34.9 & 55 \\
Finger abduction-adduction & 8.8 & 37.8 & 6.9 & 20.7 & 5.4 & 16 \\
Palmar arching & 4.4 & 3.4 & 15.3 & 0.2 & 1.9 & 5 \\
Thumb opposition & 7.2 & 8.1 & 35.3 & 27.3 & 57.9 & 24 \\
\hline
\end{tabular}

Finally, Table 6 shows the AIM for the different artificial hands, obtained using Equation 1 and considering the parameters shown in Tables 4 and 5. Two factors affect the final AIM obtained by a hand (Equation 1): its mobility and type of actuation, represented by the number of DoF, the number of actuators, the number of digits and phalanges per digit, and the type of underactuation, affecting to the final parameters $k_{i}$; and how this 


\section{8 | Chapter 4}

mobility and actuation system is distributed among the different groups of DOFs, with regard to the human hand, affecting through the weighting factor $w_{i}$ (Table 4). The most advanced robotic hands (DLR/HIT II and Shadow) with a significant amount of motors and DoFs, and located in the important groups of DoFs, with higher weight $w_{i}(\mathrm{~F} / \mathrm{E}$, T.OPP, and AB/AD), obtained the highest AIM scores, above 75\%. The commercial prosthetic hands iLimb and Bebionic as well as some 3D-printed hands (ADA, IMMA) obtained AIM scores between $40 \%$ and $50 \%$. These hands include a reasonable number of motors and DoFs in the important groups (F/E and T.OPP). The rest of the hands obtained scores below $40 \%$ with the lowest score being for the SensorHand. The reason behind this lower AIM is an improvable number of DoFs, motors, or type of underactuation in the groups of F/E, T.OPP, or both.

Table 5. Parameter $k_{i}$ (Equation 2) for each group of DoFs for the different artificial hands. F/E: finger flexion-extension, AB/AD: finger abductionadduction, P.ARC: palmar arching, T.OPP: thumb opposition.

\begin{tabular}{lcccc}
\hline Artificial hand & F/E & AB/AD & P.ARC & T.OPP \\
\hline IMMA & 0.48 & 0.25 & 0 & 0.50 \\
Cyborg Beast & 0.29 & 0 & 0 & 0.18 \\
Flexy-Hand & 0.39 & 0.25 & 0 & 0.23 \\
KIT & 0.47 & 0 & 0 & 0.35 \\
ADA & 0.58 & 0.25 & 0 & 0.40 \\
i-Limb & 0.58 & 0 & 0 & 0.55 \\
Bebionic & 0.50 & 0 & 0 & 0.50 \\
SensorHand & 0.13 & 0 & 0 & 0.10 \\
Michelangelo & 0.13 & 0 & 0 & 0.30 \\
FRH-4 & 0.46 & 0 & 0.50 & 0.40 \\
Barrett & 0.25 & 0.38 & 0 & 0.30 \\
DLR/HIT II & 0.83 & 1 & 0 & 0.70 \\
Shadow & 0.83 & 1 & 0.50 & 1 \\
\hline
\end{tabular}

The results shown in Table 6 indicate that the artificial hands analyzed in the literature with other anthropomorphism indexes, such as AI (Feix et al., 2013) or $A_{R}$ (Liarokapis et al., 2013), are ranked equally by the AIM and the other metrics, although the scores are different. The method used to compute the indexes justify these different scores. The AI is obtained from the achievable workspace of positions and orientations of the fingers' distal segments and compares this with information obtained experimentally from human hand grasping. The $A_{R}$ is based on the computation of the finger phalanx workspace combined with that of the finger base frames, and the comparison with the human hand is made through a simplified model of their 
joints and geometry. It is worth to note that obtaining $\mathrm{AI}$ and $\mathrm{A}_{\mathrm{R}}$ involves using complex algorithms and detailed information of the hand design, not easily available, while obtaining the AIM just requires information about the number of DoFs and the possibility to control them independently. Despite these differences in the method used to obtain each index, the fact that they rank equally, the hands as the AIM can be seen as a kind of validation of our index. Two main points can justify the use of the AIM as a method for evaluating the anthropomorphism of an artificial hand:

- It is really quick to obtain: simply, the parameter $k_{i}$ has to be calculated, according to the DoFs and actuation methods of the artificial hand and Equation 1 has to be applied ( $w_{i}$ is provided above).

- It analyzes not only the topology but also the functionality of the artificial hand because it takes into account the results obtained in grasping tests and ADL with the human hand.

Table 6. Results of the Anthropomorphism Index of Mobility (AIM) for different artificial hands, and comparison with other indexes of the literature.

\begin{tabular}{lccc}
\hline Artificial Hand & AIM (\%) & $\begin{array}{c}\text { AI (\%) } \\
\text { (Feix et al., } \\
\text { 2013) }\end{array}$ & $\begin{array}{c}\mathrm{A}_{\mathrm{R}}(\%) \\
\text { (Liarokapis } \\
\text { et al., 2013) }\end{array}$ \\
\hline IMMA & 42 & & \\
Cyborg Beast & 20 & & \\
Flexy-Hand & 31 & & \\
KIT & 34 & & \\
ADA & 46 & & \\
i-Limb & 45 & & \\
Bebionic & 40 & & \\
SensorHand & 10 & 0.25 & \\
Michelangelo & 14 & 2.80 & \\
FRH-4 & 37 & 5.20 & \\
Barret & 27 & & 10.38 \\
DLR/HIT II & 78 & & 26.61 \\
Shadow & 88 & & 39.93 \\
\hline
\end{tabular}

Notwithstanding, some important aspects in the design of an artificial hand are not within the scope of the AIM: the orientation of the joint axes, the range of motion of the different hand joints, the dimension of the phalanges, the friction coefficient of the parts of the hand in contact with the objects, the grasping force exerted by the actuators, the efficiency of the driving linkages, the control system, etc. Some previous studies (Andrés et al., 2018; Ceccarelli et al., 2005; Controzzi et al., 2014) have shown the 
relevance of these aspects. In this sense, the AIM, involving mainly the topological structure, the number of actuators, and the type of underactuation, can be considered as an index especially useful in the concept design stage. The other design considerations cited above should be taken into account in later design stages: preliminary or detail design. Additional indexes that take into account these aspects could be interesting, and future works can go in this way. The index proposed by Liu et al. (Liu et al., 2015) considers some of these aspects, but it does not include their relevance for functionality according to human grasping tests. With respect to the phalanx dimensions and the joints' range of motion, the authors developed some studies (Gracia-Ibáñez et al., 2017b; Llop-Harillo et al., 2018; Vergara et al., 2018) helping to obtain anthropomorphic designs. However, the evaluation of some of the design aspects cited above is difficult to be performed with indexes, requiring experimentation, after detailed design of the artificial hand and manufacturing a prototype. The authors have proposed methods for this experimental evaluation considering the main GTs in ADL and a special device for actuating the hand prototype (Llop-Harillo and Pérez-González, 2017).

The ranges of motion of the hand joints obtained in the human grasp experiment undertaken in this study are shown in Table 7. A wide range of motion for the different joints was covered with the objects selected in comparison to the functional range of motion of the human hand joints in ADL (Gracia-Ibáñez et al., 2017b). These ranges could be considered as a minimum for prostheses with functional grasping for the main GTs, although general manipulation would recommend using larger ranges if possible.

Table 7. Range of motion of the hand joints (in degrees) obtained in the human grasp experiment (CMC: carpometacarpal joint, MCP: metacarpophalangeal joint, PIP: proximal interphalangeal joint, DIP: distal interphalangeal joint, IP: interphalangeal joint, F: flexion (+)/extension (-), A: abduction (+)/adduction (-), $\mathrm{P}$ : percentile).

\begin{tabular}{|c|c|c|c|c|c|c|c|c|c|c|c|c|c|c|c|}
\hline & \multicolumn{4}{|c|}{ Thumb $\left(^{\circ}\right)$} & \multicolumn{3}{|c|}{ Index $\left(^{\circ}\right)$} & \multicolumn{2}{|c|}{ Middle $\left(^{\circ}\right)$} & \multicolumn{3}{|c|}{ Ring $\left(^{\circ}\right)$} & \multicolumn{3}{|c|}{ Little $\left(^{\circ}\right)$} \\
\hline & F & A & F & $\mathrm{F}$ & $\mathrm{F}$ & A & $\mathrm{F}$ & F & F & $\mathrm{F}$ & A & $\mathrm{F}$ & $\mathrm{F}$ & A & $\mathrm{F}$ \\
\hline & CMC & CMC & МСР & IP & MCP & MCP & PIP & MCP & PIP & МCP & MCP & PIP & MCP & MCP & PIP \\
\hline Min & -27 & 0 & -24 & -32 & -22 & -9 & 0 & -16 & 0 & -13 & -7 & 1 & -13 & -7 & -2 \\
\hline Max & 32 & 28 & 13 & 42 & 51 & 24 & 62 & 65 & 66 & 68 & 16 & 76 & 69 & 12 & 68 \\
\hline P5 & -6 & 0 & -10 & -5 & -4 & -3 & 1 & -1 & 1 & -1 & -1 & 1 & -3 & -1 & 0 \\
\hline P95 & 15 & 19 & 2 & 17 & 30 & 9 & 36 & 40 & 42 & 30 & 8 & 49 & 26 & 7 & 40 \\
\hline
\end{tabular}

This study was primarily focused on prosthetic hands, and therefore, the scoring system takes into account the capability of the hand to perform the 
most important GTs for a nondominant hand to reinforce bimanual grasping (through parameter $z_{g}$ ). For the case of a dominant hand reinforcing bimanual grasping, the parameter $z_{g}$ for the four GTs considered in this study changes to (Gracia-Ibáñez et al., 2018) PP (58.0\%), EG (16.6\%), TP (9.5\%), and TVG (16.0\%). The effect of this change on the resulting $w_{i}$ is negligible and implies a disparity of the AIM obtained for the artificial hands analyzed (Table 6) of a maximum of $1 \%$. Therefore, the AIM is considered useful to evaluate the anthropomorphism of both dominant and nondominant hands. With this result and the result obtained from the comparison of the AIM with other indexes of the literature (Feix et al., 2013; Liarokapis et al., 2013) we can conclude that the index proposed can be valid for artificial both robotic and prosthetic hands, regardless of whether they are dominant or nondominant hands.

\section{Conclusion}

In this study, we have presented an anthropomorphism index (AIM) that can be used to evaluate and compare the mobility of artificial hands in relation to the human hand functionality, especially in concept design. The AIM evaluates the topology of the whole hand (joints and DoFs) and the possibility to control these DoFs independently according to their functionality. We have shown that the index can be valid for both prosthetic and robotic hands, dominant and nondominant hands. To define the index, the functionality of the different groups of DoFs of the hand $(\mathrm{F} / \mathrm{E}, \mathrm{AB} / \mathrm{AD}$, P.ARC, and T.OPP) was analyzed according to a human grasp experiment on twenty subjects with the four main GTs for personal autonomy in ADL. It was concluded that the relevance of the different groups of DoFs $\left(w_{i}\right)$ was $55 \%$ for F/E, $16 \%$ for AB/AD, 5\% for P.ARC and 24\% for T.OPP. Thirteen artificial hands, including affordable 3D-printed prosthetic hands, advanced commercial prosthetic hands, and robotic hands, were evaluated and compared with the AIM, and the reason for their differences was discussed. The results obtained in this study should be taken into account in the concept design stage of new prototypes in order to obtain new designs that maximize their functionality. Further research will focus on new metrics for later design stages considering other design aspects (range of motion of the joints, relative length of the phalanges, orientation of the joints axes, etc.) and on experimental benchmarks to measure the grasping capability of artificial hands. 


\section{Data Availability}

The human hand kinematics expressed as joint angles and scores referred to the five PCs, the loading matrix corresponding to these PCs, and the $c_{i j}$ values for the joints of the artificial hands analyzed; the data used to support the findings of this study are included within the supplementary information files.

\section{Acknowledgments}

This work was supported by the Spanish Ministry of Economy and Competitiveness and ESF (grant number BES-2015-076005); the Spanish Ministry of Economy and Competitiveness, AEI, and ERDF (grant numbers DPI2014-60635-R, DPI2017-89910-R); and Universitat Jaume I (grant numbers UJI-B2017-70, UJI-B2017-51).

\section{References}

Andrés, F.J., Pérez-González, A., Rubert, C., Fuentes, J., Sospedra, B., 2018. Comparison of Grasping Performance of Tendon and Linkage Transmission Systems in an Electric-Powered Low-Cost Hand Prosthesis. Journal of Mechanisms and Robotics, 11(1), 11018.

Barrett Technology, 2013. BarrettHand. https://www.barrett.com/aboutbarretthand [3.01.19].

Belter, J.T., Segil, J.L., Dollar, A.M., Weir, R.F., 2013. Mechanical design and performance specifications of anthropomorphic prosthetic hands: A review. The Journal of Rehabilitation Research and Development, 50(5), 599-617.

Birglen, L., Gosselin, C., Laliberté, T., 2008. Underactuated Robotic Hands. Springer.

Calli, B., Walsman, A., Singh, A., Srinivasa, S., Abbeel, P., Dollar, A.M., 2015. Benchmarking in Manipulation Research: Using the Yale-CMU-Berkeley Object and Model Set. IEEE Robotics \& Automation Magazine, 22(3), 36-52.

Ceccarelli, M., Rodríguez, N.E.N., Carbone, G., Lopez-Cajùn, C., 2005. An Optimal Design of Driving Mechanism in a 1 Degree of Freedom (d.o.f.) Anthropomorphic Finger. Applied Bionics and Biomechanics, 2(2), 103-110.

Controzzi, M., Peccia, C., Oddo, C.M., Carrozza, M.C., Cipriani, C., 2014. Bioinspired fingertip for anthropomorphic robotic hands. Applied Bionics and Biomechanics, 11(1-2), 25-38.

Cornwall, M.W., 2007. Prosthetics and Patient Management: A Comprehensive Clinical Approach. Physical Therapy, 87(4), 482-482. 
Feix, T., Romero, J., Ek, C.H., Schmiedmayer, H.B., Kragic, D., 2013. A metric for comparing the anthropomorphic motion capability of artificial hands. IEEE Transactions on Robotics, 29(1), 82-93.

Gaiser, I., Schulz, S., Kargov, A., Klosek, H., Bierbaum, A., Pylatiuk, C., Oberle, R., Werner, T., Asfour, T., Bretthauer, G., Dillmann, R., 2008. A new anthropomorphic robotic hand, in: Humanoids 2008 - 8th IEEE-RAS International Conference on Humanoid Robots. IEEE, pp. 418-422.

Gracia-Ibáñez, V., Sancho-Bru, J.L., Vergara, M., 2018. Relevance of grasp types to assess functionality for personal autonomy. Journal of Hand Therapy, 31(1), 102-110.

Gracia-Ibáñez, V., Vergara, M., Buffi, J.H., Murray, W.M., Sancho-Bru, J.L., 2017a. Across-subject calibration of an instrumented glove to measure hand movement for clinical purposes. Computer Methods in Biomechanics and Biomedical Engineering, 20(6), 587-597.

Gracia-Ibáñez, V., Vergara, M., Sancho-Bru, J.L., Mora, M.C., Piqueras, C., 2017b. Functional range of motion of the hand joints in activities of the International Classification of Functioning, Disability and Health. Journal of Hand Therapy, 30(3), 337-347.

Gracia Ibañez, V., 2016. Contribution to hand functional assessment based on its kinematics. Universitat Jaume I, Castelló de la Plana.

Gyrobot, 2014. Flexy-Hand 2. https://www.thingiverse.com/thing:380665 [3.01.19].

Kapandji, I.A., 1982. The Physiology of the Joints: Upper limb. Churchill Livingstone.

Liarokapis, M. V., Artemiadis, P.K., Kyriakopoulos, K.J., 2013. Quantifying anthropomorphism of robot hands, in: 2013 IEEE International Conference on Robotics and Automation. IEEE, pp. 2041-2046.

Liu, H., Wu, K., Meusel, P., Seitz, N., Hirzinger, G., Jin, M.H., Liu, Y.W., Fan, S.W., Lan, T., Chen, Z.P., 2008. Multisensory five-finger dexterous hand: The DLR/HIT hand II, in: 2008 IEEE/RSJ International Conference on Intelligent Robots and Systems, IROS.

Liu, Y., Yang, D., Jiang, L., Liu, H., 2015. A synthetic framework for evaluating the anthropomorphic characteristics of prosthetic hands, in: 2015 IEEE International Conference on Advanced Intelligent Mechatronics (AIM). IEEE, pp. 877-884.

Llop-Harillo, I., Gracia-Ibáñez, V., Pérez-González, A., 2018. Analysis of anthropometric dimensions and joints range of motion of the human hand for application to the design of hand prostheses, in: 8th World Congress on Biomechanics. Dublin.

Llop-Harillo, I., Pérez-González, A., 2017. System for the experimental evaluation 


\section{4 | Chapter 4}

of anthropomorphic hands. Application to a new 3D-printed prosthetic hand prototype. International Biomechanics, 4(2), 50-59.

Llop-Harillo, I., Pérez-González, A., Cantero-Ramis, J., 2019. Analysis of motion synergies in grasping tasks for a 6-DOF tendon-driven prosthetic hand, in: 16th International Symposium on Computer Methods in Biomechanics and Biomedical Engineering and 4th Conference on Imaging and Visualization.

Llop-Harillo, I., Pérez-González, A., Gracia-Ibáñez, V., 2017. Anthropomorphism Index of Mobility for hand prostheses, in: XXVI Congress of the International Society of Biomechanics. Brisbane.

Open Bionics, 2018. Open Bionics Hands. https://openbionicslabs.com/downloads [3.01.19].

Ottobock, 2017. Ottobock myoelectric prosthetics. https://www.ottobockus.com/prosthetics/upper-limb-prosthetics/solutionoverview/myoelectric-prosthetics/ [3.01.19].

Patel, V., Burns, M., Chandramouli, R., Vinjamuri, R., 2017. Biometrics Based on Hand Synergies and Their Neural Representations. IEEE Access, 5, 1342213429.

Sancho-Bru, J.L., 2000. Model biomecànic de la mà orientat al disseny d'eines manuals. Ph.D.

Shadow Robot Company, 2019. Shadow Dexterous Hand. https://www.shadowrobot.com/products/dexterous-hand/ [3.01.19].

ten Kate, J., Smit, G., Breedveld, P., 2017. 3D-printed upper limb prostheses: a review. Disability and Rehabilitation: Assistive Technology, 12(3), 300-314.

Touch Bionics, 2019. Touch Bionics products. http://touchbionics.com/products [3.01.19].

Ullman, D.G., 2010. The Mechanical Design Process, 4th Edition, McGraw-Hill.

Vergara, M., Agost, M.J., Gracia-Ibáñez, V., 2018. Dorsal and palmar aspect dimensions of hand anthropometry for designing hand tools and protections. Human Factors and Ergonomics in Manufacturing \& Service Industries.

Weiner, P., Starke, J., Hundhausen, F., Beil, J., Asfour, T., 2018. The KIT Prosthetic Hand: Design and Control, in: IEEE/RSJ International Conference on Intelligent Robots and Systems (IROS).

World Health Organization, 2002. Towards a Common Language for Functioning, Disability and Health ICF The International Classification of Functioning, Disability and Health. World Health Organization Geneva.

Yancosek, K.E., Mullineaux, D.R., 2011. Stability of handwriting performance following injury-induced hand-dominance transfer in adults: A pilot study. The Journal of Rehabilitation Research and Development. 
Zuniga, J., Katsavelis, D., Peck, J., Stollberg, J., Petrykowski, M., Carson, A., Fernandez, C., 2015. Cyborg beast: A low-cost 3d-printed prosthetic hand for children with upper-limb differences. BMC Research Notes.

\section{Supplementary Materials}

The excel file "JointAngles" contains the joint angles for each instant for the twelve tasks repeated three times by twenty subjects during the human grasp experiment.

The excel file "Scores" contains the human hand kinematics in the human grasp experiment transformed to scores $f_{t k}$ referred to five functional synergies for the twelve tasks repeated three times by twenty subjects.

The excel file "LoadingsMatrix" contains the loadings $l_{i j k}$ of the five functional synergies during ADL.

The excel file "ArtificialHands" contains the value $c_{i j}$ according to the method of actuation for each DoF in the artificial hands analyzed and the corresponding $k_{i}$ of each hand.

\section{Available at:}

https://www.hindawi.com/journals/abb/2019/7169034/\#supplementarymaterials

Loadings Matrix:

\begin{tabular}{cccccc}
\hline & PC1 & PC2 & PC3 & PC4 & PC5 \\
\hline CMC1_F & 0.033 & 0.110 & 0.127 & -0.162 & -0.873 \\
CMC1_A & 0.166 & 0.019 & 0.782 & 0.024 & -0.021 \\
MCP1_F & 0.184 & -0.134 & -0.328 & 0.658 & 0.135 \\
IP1_F & 0.044 & 0.186 & 0.310 & -0.224 & 0.516 \\
MCP2_F & 0.336 & 0.311 & 0.298 & 0.739 & -0.021 \\
MCP2_A & 0.170 & -0.311 & -0.026 & -0.661 & 0.118 \\
PIP2_F & 0.352 & 0.264 & 0.443 & -0.098 & 0.389 \\
MCP3_F & 0.362 & 0.554 & 0.424 & 0.496 & 0.138 \\
PIP3_F & 0.898 & 0.107 & 0.085 & 0.002 & 0.116 \\
MCP4_F & 0.424 & 0.684 & 0.335 & 0.310 & 0.118 \\
MCP4_A & -0.118 & 0.869 & -0.125 & 0.095 & -0.011 \\
PIP4_F & 0.950 & 0.130 & -0.019 & 0.065 & 0.004 \\
CMC5_F & -0.260 & -0.187 & 0.668 & 0.006 & 0.050 \\
MCP5_F & 0.520 & 0.660 & 0.198 & 0.169 & 0.082 \\
MCP5_A & 0.230 & 0.909 & -0.152 & 0.055 & -0.014 \\
PIP5_F & 0.852 & 0.089 & -0.057 & 0.146 & -0.064 \\
\hline
\end{tabular}


Artificial Hands:

\begin{tabular}{|c|c|c|c|c|c|c|c|c|c|c|c|c|c|}
\hline \multicolumn{14}{|c|}{$\mathrm{C}_{\mathrm{ii}}$} \\
\hline & IMMA & CYBORG & FLEXY & KIT & ADA & ILIMB & BEBIONIC & SENSOR & MICHELANGELO & FRH4 & BARRETT & DLR & SHADOW \\
\hline MCP2_Flexion & 1 & 0.5 & 0.5 & 1 & 1 & 1 & 1 & 0.5 & 0.5 & 1 & 1 & 1 & 1 \\
\hline PIP2_Flexion & 0.75 & 0.375 & 0.375 & 0.75 & 0.75 & 0.75 & 0.5 & 0 & 0 & 1 & 0.5 & 1 & 1 \\
\hline DIP2_Flexion & 0.5625 & 0 & 0.28125 & 0 & 0 & 0 & 0 & 0 & 0 & 0 & 0 & 0.5 & 0.5 \\
\hline MCP3_Flexion & 0.5 & 0.5 & 0.5 & 0.75 & 1 & 1 & 1 & 0.5 & 0.5 & 1 & 1 & 1 & 1 \\
\hline PIP3_Flexion & 0.375 & 0.375 & 0.375 & 0.5625 & 0.75 & 0.75 & 0.5 & 0 & 0 & 1 & 0.5 & 1 & 1 \\
\hline DIP3_Flexion & 0.28125 & 0 & 0.28125 & 0 & 0 & 0 & 0 & 0 & 0 & 0 & 0 & 0.5 & 0.5 \\
\hline MCP4_Flexion & 0.5 & 0.5 & 0.5 & 0.75 & 1 & 1 & 1 & 0.25 & 0.25 & 1 & 0 & 1 & 1 \\
\hline PIP4_Flexion & 0.375 & 0.375 & 0.375 & 0.5625 & 0.75 & 0.75 & 0.5 & 0 & 0 & 0 & 0 & 1 & 1 \\
\hline DIP4_Flexion & 0.28125 & 0 & 0.28125 & 0 & 0 & 0 & 0 & 0 & 0 & 0 & 0 & 0.5 & 0.5 \\
\hline MCP5_Flexion & 0.5 & 0.5 & 0.5 & 0.75 & 1 & 1 & 1 & 0.25 & 0.25 & 0.5 & 0 & 1 & 1 \\
\hline PIP5_Flexion & 0.375 & 0.375 & 0.375 & 0.5625 & 0.75 & 0.75 & 0.5 & 0 & 0 & 0 & 0 & 1 & 1 \\
\hline DIP5_Flexion & 0.28125 & 0 & 0.28125 & 0 & 0 & 0 & 0 & 0 & 0 & 0 & 0 & 0.5 & 0.5 \\
\hline MCP2_Abduction & 0.25 & 0 & 0.25 & 0 & 0.25 & 0 & 0 & 0 & 0 & 0 & 1 & 1 & 1 \\
\hline MCP3_Abduction & 0.25 & 0 & 0.25 & 0 & 0.25 & 0 & 0 & 0 & 0 & 0 & 0.5 & 1 & 1 \\
\hline MCP4_Abduction & 0.25 & 0 & 0.25 & 0 & 0.25 & 0 & 0 & 0 & 0 & 0 & 0 & 1 & 1 \\
\hline MCP5_Abduction & 0.25 & 0 & 0.25 & 0 & 0.25 & 0 & 0 & 0 & 0 & 0 & 0 & 1 & 1 \\
\hline CMC5_Flexion & 0 & 0 & 0 & 0 & 0 & 0 & 0 & 0 & 0 & 1 & 0 & 0 & 1 \\
\hline CMC4_Flexion & 0 & 0 & 0 & 0 & 0 & 0 & 0 & 0 & 0 & 0 & 0 & 0 & 0 \\
\hline CMC1_Flexion & 0 & 0 & 0 & 0 & 0 & 0 & 0 & 0 & 0 & 0 & 0 & 1 & 1 \\
\hline CMC1_Abduction & 0.5 & 0 & 0 & 0 & 0 & 0 & 0 & 0 & 0 & 0 & 0 & 1 & 1 \\
\hline MCP1_Flexion & 1 & 0.5 & 0.5 & 1 & 1 & 1 & 1 & 0.5 & 0.5 & 1 & 1 & 1 & 1 \\
\hline MCP1_Abduction & 0.25 & 0 & 0.25 & 0 & 0.25 & 1 & 1 & 0 & 1 & 1 & 0 & 0 & 1 \\
\hline IP1_Flexion & 0.75 & 0.375 & 0.375 & 0.75 & 0.75 & 0.75 & 0.5 & 0 & 0 & 0 & 0.5 & 0.5 & 1 \\
\hline \multicolumn{14}{|c|}{$\mathrm{k}_{\mathrm{i}}$} \\
\hline F/E & 0.48 & 0.29 & 0.39 & 0.47 & 0.58 & 0.58 & 0.50 & 0.13 & 0.13 & 0.46 & 0.25 & 0.83 & 0.83 \\
\hline $\mathrm{AB} / \mathrm{AD}$ & 0.25 & 0.00 & 0.25 & 0.00 & 0.25 & 0.00 & 0.00 & 0.00 & 0.00 & 0.00 & 0.38 & 1.00 & 1.00 \\
\hline P.ARC & 0.00 & 0.00 & 0.00 & 0.00 & 0.00 & 0.00 & 0.00 & 0.00 & 0.00 & 0.50 & 0.00 & 0.00 & 0.50 \\
\hline T.OPP & 0.50 & 0.18 & 0.23 & 0.35 & 0.40 & 0.55 & 0.50 & 0.10 & 0.30 & 0.40 & 0.30 & 0.70 & 1.00 \\
\hline
\end{tabular}




\section{Chapter 5}

Anthropomorphism Indexes of the Kinematic Chain for Artificial Hands 

This chapter corresponds exactly to the published paper: Anthropomorphism Indexes of the Kinematic Chain for Artificial Hands Immaculada Llop-Harillo, Antonio Pérez-González, and Javier AndrésEsperanza Journal of Bionic Engineering, 2020, 17, 501-511

\begin{abstract}
Anthropomorphic hands have received increasing research interest in the fields of robotics and prosthetics. But it is not yet clear how to evaluate their anthropomorphism. Similarity in the kinematic chain is essential to achieve both functionality and cosmesis. A few previous works have addressed the definition of anthropomorphism indexes, although they have some limitations in its definition. In this study, three different anthropomorphism indexes have been defined to compare the kinematic chain of artificial hands with that of the human hand. These indexes are based on the comparison of: (1) the parameters of the kinematic chain (dimensions, type of joints, orientations and ranges of motion), (2) the reachable workspace, and (3) common grasping postures. Five artificial hands with different degrees of anthropomorphism have been compared using the three Anthropomorphism Indexes of the Kinematic Chain (AIKC). The results show a high correlation between the first and third AIKC for the hands compared. The second AIKC presents much lower values than the other two, although they are higher for hands that combine abduction/adduction and flexion/extension movements in the kinematic chain of each finger. These indexes can be useful during the initial stage of designing artificial hands or evaluating their anthropomorphism.
\end{abstract}

\title{
Keywords
}

Anthropomorphism, grasping, indexes, kinematics, multifingered hands

\section{Introduction}

Research interest in anthropomorphic hands has increased recently, fostered by the needs imposed by humanoid robotics and human-robot cooperation (Kemp et al., 2007; Liarokapis et al., 2012; Parada Puig et al., 


\section{0 | Chapter 5}

2008) and also by hand prosthetics (Belter et al., 2013). The popularization of additive manufacturing has also boosted the number of low-cost proposals for prosthetic applications (ten Kate et al., 2017), promoted by initiatives such as e-NABLE (http://enablingthefuture.org/), the Open Hand Project (Gibbard, 2013) and Open Bionics (Gibbard and Payne, 2018). However, according to several studies (Duong et al., 2017; Li et al., 2019; OpenAI et al., 2018; Simon et al., 2019), the degree of functionality of the human hand $(\mathrm{HH})$ is far from being achieved even by expensive commercial prostheses, such as i-Limb (David Gow, n.d.), or sophisticated robotic hands such as Shadow Dexterous Hand (Greenhill and Elias, 2015).

At the same time, there are no methods to assess this anthropomorphism and even an agreement on the meaning of this concept is lacking. Anthropomorphism can be understood as similarity to the $\mathrm{HH}$ in terms of size, weight, shape, appearance, temperature, etc., i.e. cosmesis (ten Kate et al., 2017), or as similarity in terms of functionality or dexterity (Liarokapis et al., 2012). The kinematic chain (KC) of the artificial hand (AH) is of primary importance to achieve anthropomorphism in both senses: cosmesis and functionality. The whole $\mathrm{KC}$ of a hand is composed of as many open $\mathrm{KCs}$ as there are fingers. The length of the finger segments or links and the position and orientation of their joints determine the postures that the hand can adopt, or in robotic terminology, the reachable workspace (Craig, 2004). The maximization of the degree of anthropomorphism of the $\mathrm{KC}$ of an $\mathrm{AH}$ during the design process depends, primarily, on having adequate metrics or indexes that allow its quantification.

Some previous works have attempted to quantify hand anthropomorphism. In (Biagiotti et al., 2003) a method for calculating an anthropomorphism index (AI) was proposed, based on weighting the qualitative evaluation of three aspects of the $\mathrm{AH}$ in comparison to the $\mathrm{HH}$ : the KC, the contact surfaces, and the dimensions. This method was used, together with a potential dexterity index considering the control system and sensors, to compare different anthropomorphic hands. The main limitation of this approach is the dependence on qualitative assessments. A similar approach was proposed in (Jiang et al., 2018) based on the evaluation of twelve aspects related to mechanics, rehabilitation and anatomy. The attainable grasp gestures and the distribution of the rotation axes were considered relevant aspects for anthropomorphism in that study. Recently, the authors also defined an Anthropomorphism Index of Mobility (AIM) (Llop-Harillo et al., 2019) based on the topology of the whole hand, joints and degrees of freedom (DoFs), and the possibility of controlling these DoFs 
independently (number of actuators and type of underactuation). The weighting factors to compute the AIM depend on the relevance of the different groups of DoFs of the hand according to a human grasp experiment on the most important grasp types. The computation of AIM is straightforward and useful in the concept design stage, but it does not consider some aspects like the orientation of the joint axes, the range of motion of the joints or the dimension of the phalanges. Feix et al. (Feix et al., 2013) proposed an AI based on comparing the space of positions and orientations attainable by the fingertips of the $\mathrm{AH}$ and $\mathrm{HH}$. Given the high number of parameters involved in the comparison, they used dimensional reduction techniques to define the index. Despite pioneering the methods for comparing $\mathrm{KC}$ among hands, it has the limitation of considering only the fingertips to define the workspace of the hand. In a similar approach, Liarokapis et al. (Liarokapis et al., 2013) included more points of the hand in the comparison and also the orientations of the finger base frame. However, they used convex-hulls to compare the workspaces and Euler angles to compare the orientations, which can introduce some errors in the comparison, as explained later in this paper.

The aim of the present study is to define and compare new indexes to measure the anthropomorphism of the $\mathrm{KC}$ of an $\mathrm{AH}$, while attempting to solve some of the limitations observed in AIs defined in the literature. The new indexes are intended to be quantitative and calculable from the basic information about the KC. They will be defined to range between 0 (low anthropomorphism) and 1 (HH, or a perfect mimicry of it). The KC will be compared here in terms of the length of the links and the position, orientation, and mobility of the joints.

\section{Kinematic chain description}

\subsection{Kinematic chain of the human hand}

The KC of each digit of the HH (Figure 1) is composed of four segments, in proximal to distal order: metacarpal (MC), proximal phalanx (PP), medial phalanx (MP) and distal phalanx (DP), except for the thumb, which lacks the MP. The MCs of the long fingers constitute the palm of the hand and have scarce mobility between them, although the carpometacarpal joints (CMC) have increasing mobility from the index to the little finger, thereby providing the palmar arching (Kapandji, 1982). The CMC joint of the thumb has the highest mobility, with two rotational DoFs around two nearly perpendicular 


\section{2 | Chapter 5}

axes, which enable the opposition of the thumb to the long fingers (Kapandji, 1982; Santos and Valero-Cuevas, 2006). The metacarpophalangeal joints (MCP) also have two rotational DoFs with nearly perpendicular axes: flexion/extension (F/E) in the sagittal plane and abduction/adduction (Ab/Ad) in the frontal plane (Kapandji, 1982). Finally, the proximal interphalangeal joints (PIP), the distal interphalangeal joints (DIP) of the long fingers, and the interphalangeal joint (IP) of the thumb have one DoF in the sagittal plane that enables their F/E. Altogether, the $\mathrm{KC}$ of the hand exhibits 25 DoFs, five per finger, or 23 DoFs if the mobility of the CMC joints of the index and middle fingers is neglected, as in some previous models (Sancho-Bru, 2000).

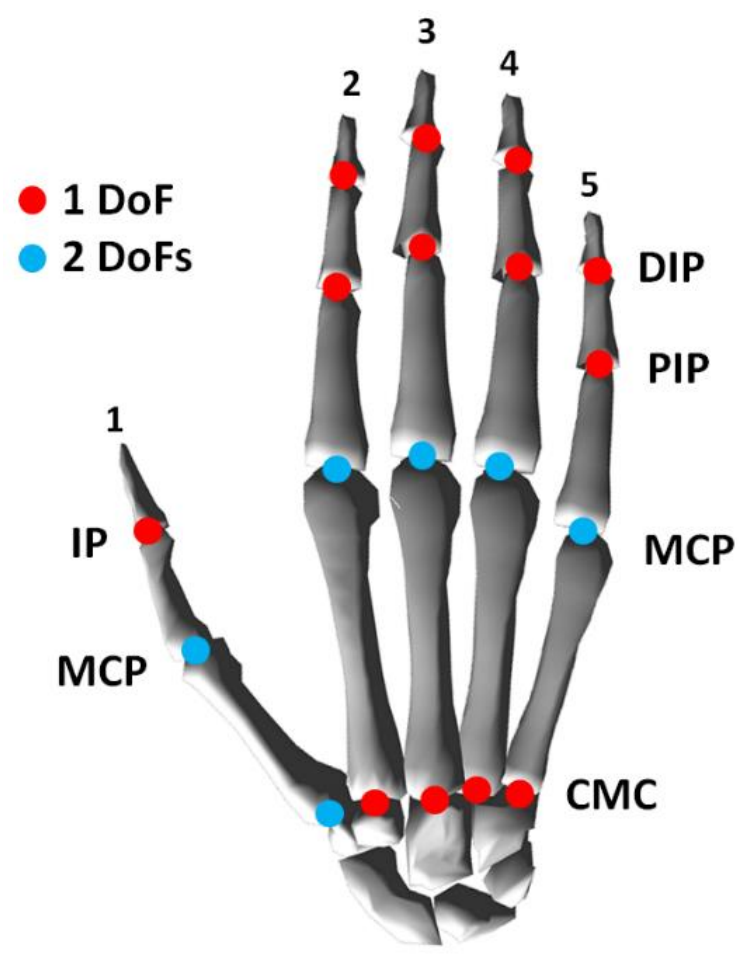

Figure 1. Kinematic chain of the human hand. The abbreviations of the joints and their DoFs are indicated.

In this work, we assume a fixed position of the rotational axes with respect to the bones. Moreover, the joints with two DoFs are considered as universal joints (perpendicular axes that intersect), although according to some studies, the axes of the joints with two DoFs are neither exactly perpendicular to each other nor do they intersect at the same point, with variations from one person to another (Santos and Valero-Cuevas, 2006). These simplifications are common for most biomechanical models of the hand (Cobos et al., 2008; León et al., 2014; Peña-Pitarch et al., 2014; Sancho-Bru, 2000) and imply a small error in the reachable positions. 


\subsection{Definition of the kinematic chain}

In order to define the $\mathrm{KC}$ of a hand, some simplifications are considered: the links are rigid and their transversal dimensions neglected; joint mobility is independent of the finger posture; and motion coupling among joints imposed by the actuation systems is obviated. A local coordinate system (LCS) is associated with each segment (bone in the case of the $\mathrm{HH}$ ) and the position and orientation of the joints are related to these LCSs. In robotics, the Denavit-Hartenberg (DH) convention (Hartenberg and Denavit, 1955) is commonly used to define the $\mathrm{KC}$ of a serial robot. It allows the $\mathrm{KC}$ to be defined with a minimum of four parameters for each segment of the chain: two rotations and two translations. However, the convention is ambiguous if the precise way of locating the positive direction of the axes is not defined and presents more than one possible solution in the case of parallel axes. Additionally, the use of this convention in the case of consecutive joints with coplanar and almost parallel rotation axes causes the location of the origin of each LCS not to match the center of the real joints. For example, in the $\mathrm{HH}$, if we assume the flexion axis of the wrist and the flexion axis of the CMC joint of the little finger as two consecutive Z-axes of the serial chain, the DH convention will locate the LCS of the latter far from the metacarpal bone axis. In sum, although the DH convention may define the chain with a minimum number of parameters, it has the disadvantage that some of them are not very intuitive from an anatomical or topological point of view.

Therefore, in this study we propose to locate the LCS for each segment of the $\mathrm{KC}$ in the center of the joint with the previous segment. Figure 2 shows the orientation of the LCSs associated with the KC of the index finger and thumb for a right hand. Specifically, those LCSs were defined with the following criteria: Z-axis coincident with the F/E axis of the joint, and directed so that flexion corresponds to a positive rotation around Z; X-axis pointing in the palmar-dorsal direction, thus indicating the $\mathrm{Ab} / \mathrm{Ad}$ axis of the joint; and Y-axis defining a right-handed trihedron, pointing towards the distal end of the fingers. In this way, an LCS exhibits positive Y-values with respect to another preceding (more proximal) LCS. This criterion is similar to that proposed by the International Society of Biomechanics (Wu and Cavanagh, 1995), with the difference that $\mathrm{X}$ and $\mathrm{Y}$ axes have opposite directions.

The fixed LCS for all the digits is located at the center of the wrist, see $\left\{\mathrm{LCS}_{0}\right\}$ in Figure 2. The reference posture (RP) of the hand is defined as that, within the range of mobility of the hand, corresponding to the smallest 


\section{4 | Chapter 5}

possible absolute $\mathrm{X}$ coordinate (expressed in $\left\{\mathrm{LCS}_{0}\right\}$ ) for the origin of every LCS. The KC of each digit is defined by means of three displacements and three rotations of each LCS with respect to its immediate proximal in the chain in the RP. Specifically, the LCS of segment $j$ (1: MC, 2: PP, 3: MP, 4: DP, 5: Fingertip) of digit $i$ (1:thumb, 2:index, 3:middle, 4:ring, 5:little) is defined with a six-element vector:

$$
\boldsymbol{v}_{i, j}=\left[\begin{array}{llllll}
x_{i, j} & y_{i, j} & z_{i, j} & \theta_{x_{i, j}} & \theta_{y_{i, j}} & \theta_{z_{i, j}}
\end{array}\right], \quad i=1,2, \ldots, 5 ; j=1,2, \ldots, 5
$$

where the first three elements of the vector correspond to the translation of $L C S_{i, j}$ with respect to $L C S_{i, j-1}$ and the last three correspond to the Euler angles necessary to orient $L C S_{i, j-1}$ as $L C S_{i, j}$, with the order of rotations XZY. For convenience, one additional LCS is associated to each fingertip ( $j=5)$; see Figure 2. For digits with four segments (such as the thumb of the $\mathrm{HH}$ ), the MP is considered inexistent, the $L C S_{i, 3}$ corresponds to the DP and the $L C S_{i, 4}$ corresponds to the fingertip.

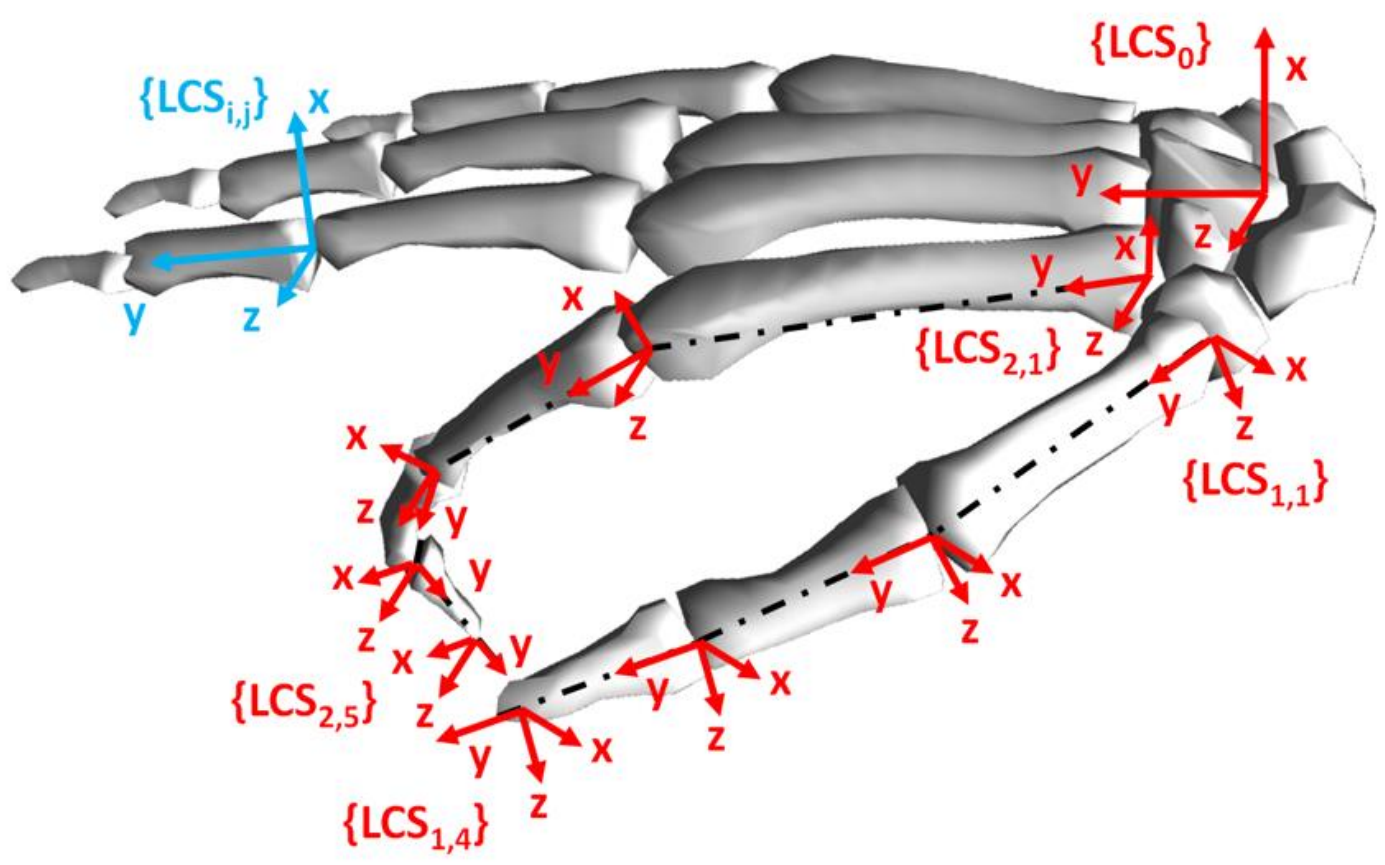

Figure 2. Proposal for the location of the $L C S_{i, j}$ in each significant point ( $\mathrm{j}$ ) of each digit (i) of the hand.

The homogeneous transformation matrix between two consecutive LCSs $(j-1$ and $j$ ) in a digit $i$ can be easily obtained from Equation 1 in the RP. For a different posture $(p)$, this transformation (Equation 2) will also be affected by the angles rotated in the joint between the two segments $\left(\beta_{i j} \mathrm{~F} / \mathrm{E}, \alpha_{i j}\right.$ $\mathrm{Ab} / \mathrm{Ad})$ : 


$$
T_{i, j}^{p}=\left[\begin{array}{cc}
R_{i, j}^{p} & t_{i, j} \\
\mathbf{0} & 1
\end{array}\right]
$$

where:

$$
\begin{gathered}
\boldsymbol{R}_{\boldsymbol{i}, j}^{\boldsymbol{p}}=\boldsymbol{R}_{\boldsymbol{x}}\left(\theta_{x_{i, j}}\right) \cdot \boldsymbol{R}_{\boldsymbol{z}}\left(\theta_{z_{i, j}}\right) \cdot \boldsymbol{R}_{\boldsymbol{y}}\left(\theta_{y_{i, j}}\right) \cdot \boldsymbol{R}_{\boldsymbol{x}}\left(\alpha_{i j}\right) \cdot \boldsymbol{R}_{\mathbf{z}}\left(\beta_{i j}\right) \\
\boldsymbol{t}_{\boldsymbol{i}, \boldsymbol{j}}=\left[\begin{array}{lll}
x_{i, j} & y_{i, j} & z_{i, j}
\end{array}\right]^{T} \\
\boldsymbol{R}_{\boldsymbol{x}}(\theta)=\left[\begin{array}{ccc}
1 & 0 & 0 \\
0 & \cos \theta & -\sin \theta \\
0 & \sin \theta & \cos \theta
\end{array}\right], \boldsymbol{R}_{\boldsymbol{y}}(\theta)=\left[\begin{array}{ccc}
\cos \theta & 0 & \sin \theta \\
0 & 1 & 0 \\
-\sin \theta & 0 & \cos \theta
\end{array}\right], \boldsymbol{R}_{\boldsymbol{z}}(\theta)=\left[\begin{array}{ccc}
\cos \theta & -\sin \theta & 0 \\
\sin \theta & \cos \theta & 0 \\
0 & 0 & 1
\end{array}\right]
\end{gathered}
$$

The absolute position of the $L C S_{i, j}$, associated to segment $j$ of digit $i$, with respect to the fixed system $\left\{\mathrm{LCS}_{0}\right\}$ located on the wrist, is obtained by successive multiplication of the transformation matrices of the consecutive segments:

$$
T_{o_{-} i, j}^{p}=T_{i, 1}^{p} \cdot \ldots \cdot T_{i, j}^{p}
$$

\section{Anthropomorphism index of the kinematic chain (AIKC)}

To define an AIKC it is necessary to compare the $\mathrm{KC}$ of the $\mathrm{AH}$ with that of the $\mathrm{HH}$. The features involved in this comparison are the dimensions and the topology of the KC. The latter includes not only the type of joints and their orientation, but also their ranges of motion. These features determine the postures that the hand can achieve, and therefore influence its grasping ability and manipulability. Different alternatives to define this index are proposed below. In all of them the index is defined so that it ranges from 0 (low anthropomorphism) to 1 ( $\mathrm{HH}$, or a perfect mimicry of it).

\section{1. $A I K C_{1}$ : based on the parameters of the kinematic chain}

A first strategy for comparing hands consists in defining a vector of characteristic parameters of the kinematic structure of the $\mathrm{AH}$ and comparing it with its corresponding vector of the $\mathrm{HH}$. For each segment of the hand it is possible to define three characteristic vectors: one with the information about the relative position of the LCS of the segment with respect to its proximal segment (defined by Equation 4); a second vector with the orientation of the LCS with respect to its proximal segment (defined by the three Euler angles from Equation 1); and a third vector defining the limits of the rotation angles, $\beta_{i j}$ for $\mathrm{F} / \mathrm{E}$ and $\alpha_{i j}$ for $\mathrm{Ab} / \mathrm{Ad}$, that are allowed in the joints around the RP. The overall difference between these vectors in the human and the artificial models could be considered an error in the achievement of 


\section{6 | Chapter 5}

anthropomorphism. This error can be used to define a metric for the anthropomorphism of the KC.

The normalized error in the translation vectors between the $\mathrm{AH}$ and $\mathrm{HH}$ in each segment can be defined (Equation 7) as the Euclidean norm of the difference between the translation vector defined in Equation 4 for the $\mathrm{AH}$ and its corresponding one for the $\mathrm{HH}, \boldsymbol{t}_{\boldsymbol{h}, \boldsymbol{j}}$, divided by a reference value for the lengths, $L_{\text {ref. }}$ The length of the longest segment of the HH (MC bone of the index) has been taken as the reference in this study. This error value is limited to a maximum of 1 .

$$
e_{t_{i, j}}=\min \left(1,\left\|\boldsymbol{t}_{i, j}-\boldsymbol{t}_{\boldsymbol{h i}, j}\right\| / L_{r e f}\right)
$$

The error in the orientation of the LCS requires a deeper analysis, as it is not correct to obtain it from the Euclidean norm of the difference between vectors containing Euler angles (Huynh, 2009). According to (Huynh, 2009) the use of quaternions is the most correct and computationally efficient method to compare 3D rotations. Any rotation of a solid can be expressed as a rotation of angle $\theta$ around a unit vector $u_{x} \mathbf{i}+u_{y} \mathbf{j}+u_{z} \boldsymbol{k}$, and it is possible to express this rotation through the unit quaternion:

$$
\boldsymbol{q}=\cos \frac{\theta}{2}+\left(u_{x} \boldsymbol{i}+u_{y} \boldsymbol{j}+u_{z} \boldsymbol{k}\right) \sin \frac{\theta}{2}
$$

A scalar product between two unit quaternions measures the difference between the two orientations (Huynh, 2009) and has the advantage of giving a result in the range $0-1$, where 1 corresponds to two significantly different orientations and 0 corresponds to two identical orientations. Therefore, the orientation error in each segment is defined as:

$$
e_{o_{i, j}}=1-\left|\boldsymbol{q}_{\boldsymbol{i}, j} \cdot \boldsymbol{q}_{\boldsymbol{h i}, \boldsymbol{j}}\right|
$$

where $\boldsymbol{q}_{i, j}$ is the quaternion corresponding to the relative orientation between the $L C S_{i, j}$ and its preceding $L C S_{i, j-1}$ in the analyzed hand, and $\boldsymbol{q}_{\boldsymbol{h}, \boldsymbol{j}}$ is that of the $\mathrm{HH}$. These quaternions have been obtained from the rotation matrix in the RP using the Matlab function rotm2quat. By default this function always selects the quaternion with the positive scalar part between the two opposites that represent the same rotation.

Finally, the error in the mobility range of each joint $j$ for digit $i$ is obtained by averaging the normalized absolute value of the difference between the limit ranges of the $\mathrm{AH}$ and $\mathrm{HH}$ :

$$
e_{r_{i, j}}=\frac{1}{2}\left[\min \left(1, \frac{\left|r_{i, j}-r_{h i, j}\right|^{+}}{\frac{\pi}{2}}\right)+\min \left(1, \frac{\left|r_{i, j}-r_{h i, j}\right|^{-}}{\frac{\pi}{2}}\right)\right]
$$


where $r_{i, j}$ is the mobility range limit of joint $j$ of digit $i$ of the AH and $r_{h i, j}$ is that of the $\mathrm{HH}$, and the two addends correspond to the positive motion (flexion, abduction) around the RP and the negative one (extension, adduction). In order to normalize the error to a value between 0 and 1 , it is divided by $\pi / 2$ as a characteristic angle of the approximate maximum range of the hand flexion joints, limiting the maximum value of the error to 1.

Using the translation, orientation and range error values obtained with Eqs. (7), (9) and (10), the $A I K C_{1}$ of a hand is defined as:

$$
A I K C_{1}=1-\sum_{i=1}^{5} w_{i} \cdot\left[\frac{1}{n_{i}} \sum_{j=1}^{n_{i}} e_{t_{i, j}}+\frac{1}{n_{i}} \sum_{j=1}^{n_{i}} e_{o_{i, j}}+\frac{1}{m_{i}} \sum_{j=1}^{m_{i}} e_{r_{i, j}}\right] / 3
$$

where $n_{i}$ is the number of segments of digit $i$ (4 for the thumb, and 5 for the long fingers), $m_{i}$ is the number of DoFs of digit $i$ ( 5 for all the digits), and the coefficient $w_{i}$ allows a different weighting of the error in each digit. Specifically in this study, it is proposed to use $w_{1}=0.5$ for the thumb and $w_{2}=w_{3}=w_{4}=w_{5}=0.125$ for each long finger, due to the predominant weight of the thumb in manipulation (Soucacos, 2001). In cases where an AH has fewer fingers or segments than the $\mathrm{HH}$, a value of 1 should be assigned to the error associated with the missing segment in the preceding equations.

\subsection{AIKC $_{2}$ : based on the workspace}

The change in the length of the segments, the orientations of the axes or the range of mobility of the joints causes changes in the reachable workspace or, in other words, in the postures that the hand can adopt. It is possible to base the comparison of the KC on this workspace. Some previous works have used this strategy (Dermitzakis et al., 2013; Feix et al., 2013; Liarokapis et al., 2013), but with the limitations of including only the position of the fingertips (Feix et al., 2013) or considering the differences in orientation only in the MCP joints (Liarokapis et al., 2013).

The space of the positions reached by a certain point of the hand can be obtained by moving all the DoFs of the $\mathrm{KC}$ of the fingers within their range of mobility. One way to quantify this workspace is to use the concepts of convex-hull (Barber et al., 1996) or alpha-shape (Edelsbrunner, 2010). Both concepts attempt to quantify the space covered by a point cloud. To generate the alpha-shape, a spherical tool with radius alpha is moved to try to penetrate the cloud of points, which represents constraints on the movement of the tool. The space delimited by the points that impede the pass of the tool is the alpha-shape. The convex-hull is a particular case where the radius of the tool is infinite. 


\section{8 | Chapter 5}

It is possible to compare hands by comparing the alpha-shape or convexhull of the cloud of positions reachable by the tip and the centers of the joints of the digits. In this study, the use of the alpha-shape is proposed, as the convex-hull may include interior regions or other areas that do not strictly pertain to the reachable workspace. Before obtaining the alpha-shape, it is necessary to generate the space of reachable positions by moving all the DoFs of each finger within their range of mobility. In order to limit the magnitude of the problem to a reasonable number of points, we decided to generate random hand positions, moving each DoF simultaneously within its range of mobility. Through preliminary analyses, we observed that $n_{p}=10^{5}$ random postures covered the workspace reachable by the hand reasonably well, the error obtained in the value of the AI being lower than $0.1 \%$.

For the computation of $A I K C_{2}$, the following procedure was used:

- Computation of the 3D alpha-shapes of the point cloud corresponding to the origin of each $L C S_{i, j}$.

- Computation of $n_{i, j}$ as the number of points of the workspace generated by each $L C S_{i, j}$ of the $\mathrm{HH}$ that also lay within the alpha-shape of its corresponding $L C S_{i, j}$ of the $\mathrm{AH}$.

- Computation of an index for each digit $i$ as the average of the fraction $n_{i, j} / n_{p}$, across all the LCSs of that digit.

$$
i_{i}=\frac{1}{5 \cdot n_{p}} \sum_{j=1}^{5} n_{i, j}
$$

- The $A I K C_{2}$ was obtained by weighting the index of each digit with the same coefficients as in Equation 11:

$$
A I K C_{2}=\sum_{i=1}^{5} w_{i} \cdot i_{i}
$$

Matlab was used to compute $A I K C_{2}$ with the predefined functions alphaShape, inShape. For the computation of the alpha-shape for each LCS, the alpha radius was selected using the Matlab function criticalAlpha, under the condition that the alpha-shape should be defined with only one region.

\section{3. $A I K C_{3}$ : based on the comparison of common grasping postures}

A third option proposed here to define anthropomorphism is based on comparing the positions and orientations adopted by the LCSs of the AH and $\mathrm{HH}$ when grasping a set of objects that are representative of typical grasping postures employed during daily life. In this study, we propose to obtain the 
position and orientation of the LCSs by simulation using the kinematic models.

Three objects are initially proposed: a cylinder, a sphere and a prism, representative of cylindrical, spherical, and extension grips. In order to simulate each grasp, an optimization process was undertaken, with the joint angles as variables, in order to minimize an objective function that was representative of the intended type of grasp. Figure 3 shows the $\mathrm{KC}$ of the $\mathrm{HH}$ in the optimized grasping posture for each object.
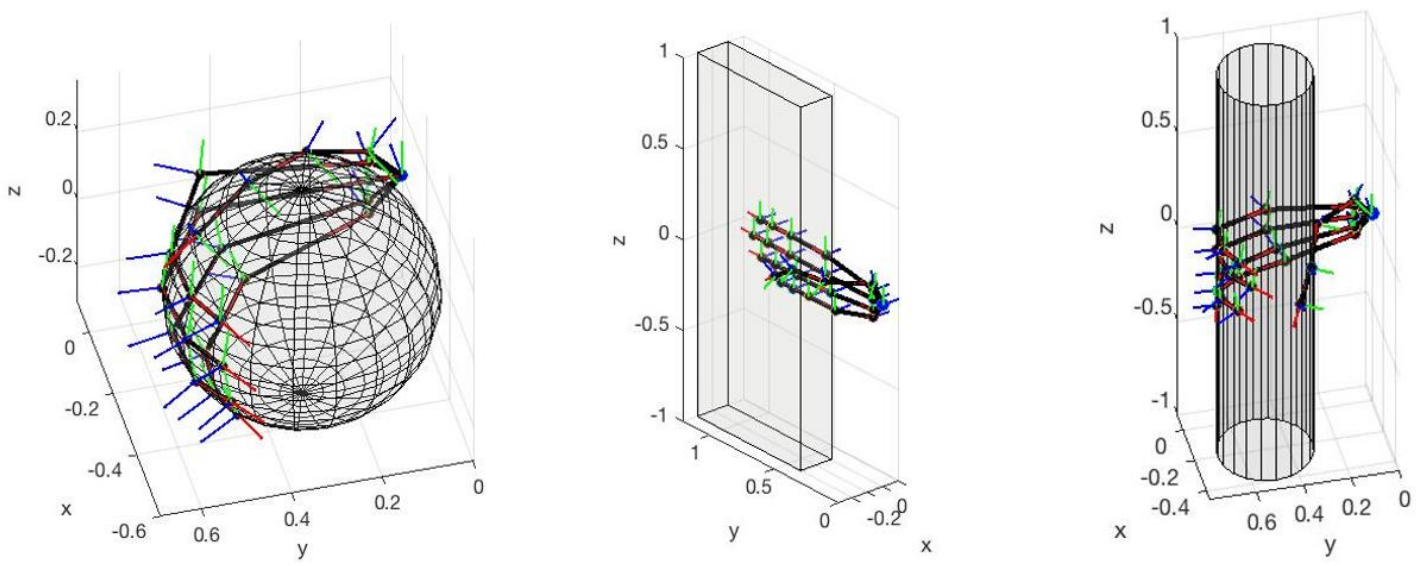

Figure 3. Grasping postures of the human hand on the three selected objects placed in the fixed $\left\{\mathrm{LCS}_{0}\right\}$ of the wrist. The LCS associated with each joint is shown: blue $\mathrm{X}$-axis, red $\mathrm{Y}$-axis, green $\mathrm{Z}$-axis.

The computation of $A I K C_{3}$ is performed following these steps:

- Simulation of the grasping postures with both HH and AH on each of the $k$ objects ( $k=1$ to $k=3$ in this study). This implies the minimization of an objective function $f_{k}$ that is different for each object (see Supplementary Materials), using the angles of $\mathrm{F} / \mathrm{E}$ and $\mathrm{Ab} / \mathrm{Ad}$ of the joints, $\beta_{i j}$ and $\alpha_{i j}$, as variables.

- Computation of the transformation matrices of each LCS with respect to the fixed one ( $\left\{\mathrm{LCS}_{0}\right\}$ in the wrist), in each grasping posture using Equation 6.

- Computation of the AI for the posture corresponding to each object $k$ using Equation 14. This is similar to Equation 11, but here the translation and orientation errors are computed from the transformations of each LCS with respect to $\left\{\mathrm{LCS}_{0}\right\}$ instead of its immediately proximal LCS in the KC, and the error in the mobility range does not apply, because one specific posture is being compared. 


$$
A I K C_{3 k}=1-\sum_{i=1}^{5} w_{i} \cdot\left[\frac{1}{n_{i}} \sum_{j=1}^{n_{i}} e_{t_{i, j}}+\frac{1}{n_{i}} \sum_{j=1}^{n_{i}} e_{o_{i, j}}\right] / 2
$$

- Computation of $\mathrm{AIKC}_{3}$ as the average across objects, namely:

$$
A I K C_{3}=\frac{1}{k} \sum_{k=1}^{k} A I K C_{3 k}
$$

\section{Evaluation of artificial hands}

In order to evaluate and compare the usefulness and effectiveness of the indexes defined here, they were computed for various AHs, using Matlab. The AHs were (Figure 4): a) Barrett (Townsend, 2013), which is a threefinger robotic gripper; b) BruJa (Andrés et al., 2019) and c) IMMA (LlopHarillo and Pérez-González, 2017), both low-cost anthropomorphic prosthetic hands designed by the authors' research group; d) Shadow hand, claimed to be the most anthropomorphic robotic hand (Greenhill and Elias, 2015); and e) i-Limb hand (David Gow, n.d.), one of the most advanced commercial prosthetic hands.

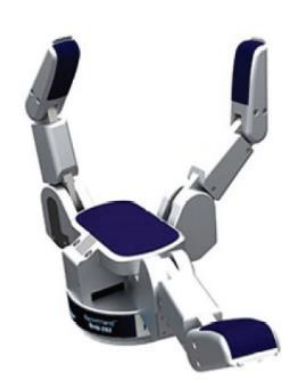

(a) Barrett $\subset$ Barrett Technology

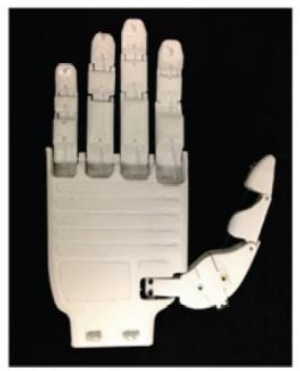

(b) BruJa

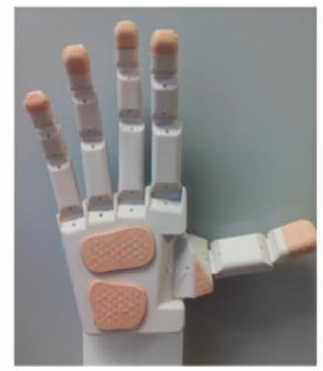

(c) IMMA

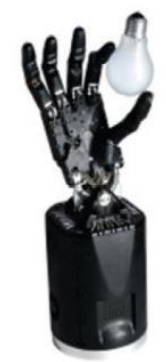

(d) Shadow CShadow Robot Company

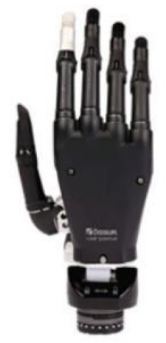

(e) i-Limb $\odot$ Össur

Figure 4. Artificial hands analyzed and compared in this study.

The data about the kinematic structure of each hand (Equation 1) and the mobility ranges in its joints are shown in the Supplementary Materials. $\mathrm{HH}$ data were obtained from previous works (León et al., 2014; Sancho-Bru, 2000), and the geometric information about the AHs was taken from the data supplied by the manufacturers or designers.

\section{Results}

Table 1 shows the AIKCs $(s=1,2,3)$ for the hands analyzed, in which value 1.0 means a perfect mimicry of the $\mathrm{HH}$ model. The Shadow hand is the most anthropomorphic and the Barrett the least anthropomorphic according to any of the indexes, although the $A I K C_{2}$ of the Barrett hand is comparable to that of the BruJa and IMMA hands. $A I K C_{1}$ and $A I K C_{3}$ results 
are much higher than those of $A I K C_{2}$. According to $A I K C_{2}$, the degree of anthropomorphism of all the hands is low, in the order of $15 \%$ for the Shadow hand, and in the order of 1-2\% for the Barrett, BruJa, IMMA, and iLimb hands. This is because the workspace of each finger is planar under the premise of considering the absence of Ab/Ad DoF in the MCP joints of these AHs. In fact, to avoid obtaining null volumes of the alpha-shape, the simulation was performed by setting an Ab/Ad range of $\pm 1^{\circ}$ in their MCP joints. This mobility could be assumed due to the flexibility of the joints themselves. As an example, Figure 5 shows the alpha-shape of the workspace associated with the LCS of every joint in the human and the IMMA hands.

Table 1. Anthropomorphism indexes obtained for each artificial hand.

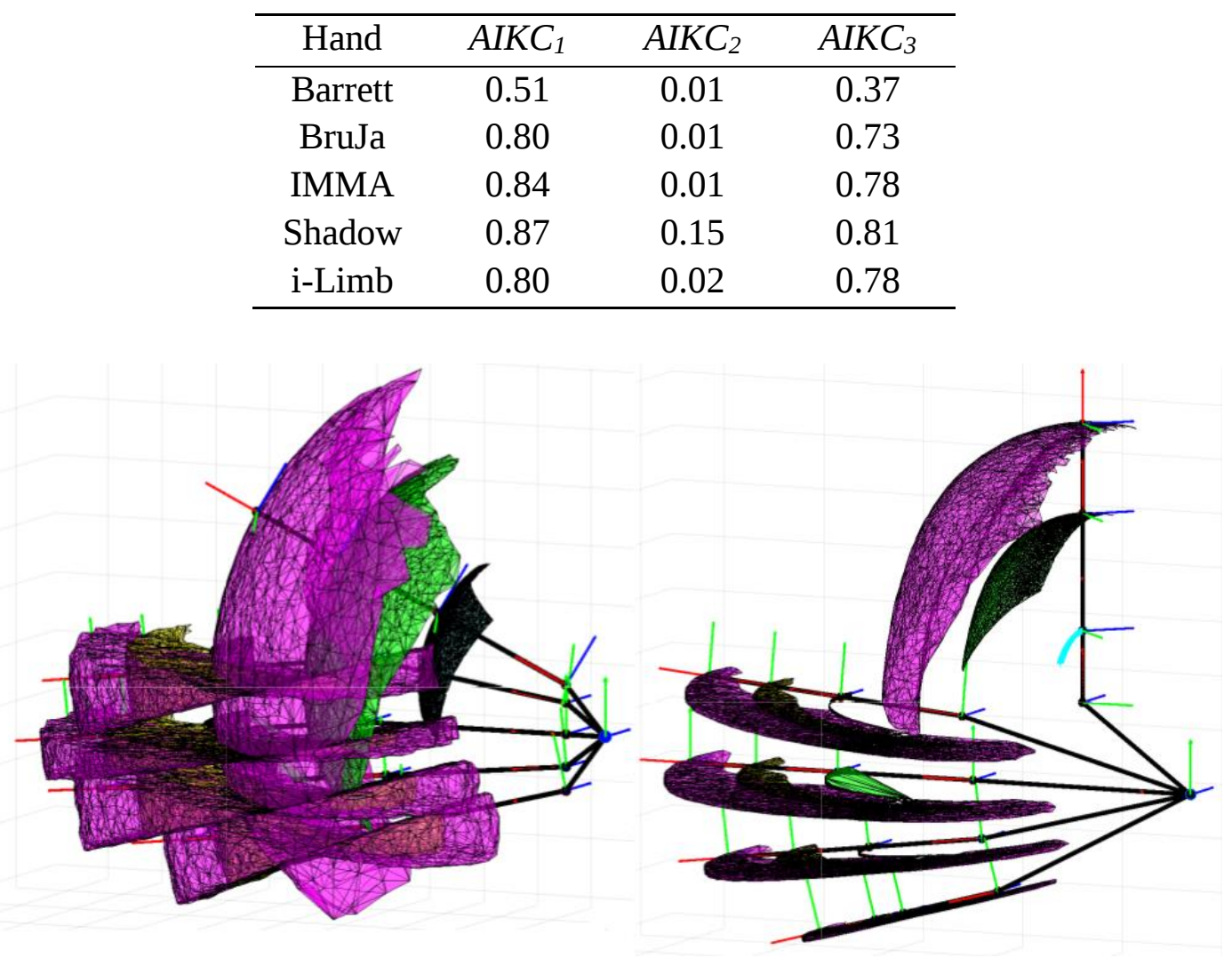

Figure 5. Alpha-shape of the human hand (left) and IMMA hand (right) workspaces for each joint (fingertip: pink, DIP: yellow, PIP and IP: pink, MCP: green).

The results shown in Table 1 also indicate a clear correlation between $A I K C_{1}$ and $A I K C_{3}$. The correlation coefficient between these indexes is higher than $99 \%$, despite the fact that $A I K C_{1}$ compares the kinematic structure and the mobility ranges, and $A I K C_{3}$ compares the $\mathrm{KC}$ but with the positions and orientations of the LCSs in grasping postures. 


\section{2 | Chapter 5}

The differences in $\mathrm{AIKC}_{3}$ across objects were small for all hands, ranging from 0.34 to 0.38 for Barrett, from 0.67 to 0.78 for BruJa, from 0.74 to 0.85 for IMMA, from 0.76 to 0.84 for Shadow, and from 0.77 to 0.81 for i-Limb.

\section{Discussion}

In this study, three indexes have been defined and compared to quantify the anthropomorphism of the KC of AHs. These indexes are straightforward computed and can be useful during the initial design stages of AHs, or to evaluate them, as performed in this study with five different AHs. They evaluate important aspects of anthropomorphism in accordance with previous studies (Feix et al., 2013; Jiang et al., 2018; Liarokapis et al., 2013), and overcome some of their limitations. Here, we considered all the parameters for defining the $\mathrm{KC}$, that is, the position and orientation of all the joints including all the finger segments. In (Feix et al., 2013) only the fingertips were considered to define the workspace of the hand and in (Liarokapis et al., 2013) the orientation differences only were considered in the MCP joints. Moreover, the orientations are compared while computing the indexes by means of quaternions (Huynh, 2009), as a more accurate alternative to Euler angles (Liarokapis et al., 2013) or rotation matrices (Feix et al., 2013). The use of the alpha-shape proposed here as an alternative to the convex-hull to compare the workspace avoids the inclusion of interior regions or other areas that do not strictly pertain to the reachable workspace (Liarokapis et al., 2013).

The low values obtained in $A I K C_{2}$ for all the AHs analyzed are explained by the much smaller workspace reachable by their finger joints as compared with the $\mathrm{HH}$ (see Figure 5). To confirm this, we analyzed a hand with the same kinematic structure as the $\mathrm{HH}$ but with the mobility range of $\mathrm{Ab} / \mathrm{Ad}$ in the MCP joints of all fingers reduced to $\pm 1^{\circ}$, resulting in an $A I K C_{2}$ of 0.07 . The combination of Ab/Ad together with $\mathrm{F} / \mathrm{E}$ movements in the same finger is what allows the $\mathrm{HH}$ to increase the volume of its workspace. Indeed, the Shadow hand achieves a higher $A I K C_{2}$ because its MCP joints have two DoFs (F/E and Ab/Ad). In the Barrett hand, the CMC joint allowing Ab/Ad in the index and middle fingers, combined with the flexion of the MCP and interphalangeal joints, compensates for the fact that the hand consists of only three fingers and achieves an $A I K C_{2}$ comparable to that of apparently more anthropomorphic hands.

Anthropomorphism estimations obtained in previous works maintain a certain correlation with those obtained in this study, despite being based on 
different definitions. For example, in Liarokapis et al. (Liarokapis et al., 2013) the total anthropomorphism score was around 10\% for Barrett and close to $40 \%$ for Shadow. These values are higher than those obtained in $A I K C_{2}$ because they are based on the comparison of workspace volumes using convex-hulls instead of alpha-shapes. Our results are not directly comparable to those of Feix et al. (Feix et al., 2013), since different hands were analyzed, although the orders of magnitude were similar to those obtained here with $A I K C_{2}$. In (Feix et al., 2013) a value of 5.2\%-9.2\% (depending on the sampling method) was obtained for the hand with the highest number of DoFs analyzed (FRH-4) and $0.25 \%$ for the prosthetic hand SensorHand ${ }^{\odot}$ (Otto Bock) with three fingers, which is comparable to the Barrett hand. In (Llop-Harillo et al., 2019) the results obtained for the AIM were: 27\% Barrett, 42\% IMMA, 45\% i-Limb, and 88\% Shadow. The results are comparable to those of the present study, in the sense that in both studies the Shadow and the Barrett hands are the most and the least anthropomorphic, respectively. However, AIKC $_{1}$ and AIM rank the IMMA and i-Limb differently. Furthermore, the Shadow hand exhibits higher AIM values (almost double) compared to the previous ones, while for the $A I K C_{1}$ and $A I K C_{3}$ these differences are lower than $10 \%$. The differences between the results of the two studies may be caused by the fact that the number of actuators and type of underactuation, considered in the AIM definition, were not taken into account in this study.

The high correlation obtained between $A I K C_{1}$ and $A I K C_{3}$ is unexpected. Although both indexes are calculated in a similar way, $A I K C_{3}$ does not include information about the mobility ranges, but only about the positions and orientations of the LCSs, with a different reference and in different postures. The correlation between the two indexes would probably decrease if the number of grasping postures increases. This point should be investigated in future works. If the level of correlation between the indexes remains high after increasing the grasping postures, this would suggest that the information they provide is redundant and therefore the use of $A I K C_{1}$ would be preferable since its calculation is more straightforward. In contrast, the information provided by indexes $A I K C_{1}$ and $A I K C_{2}$ is complementary, since hands with different kinematic structures, with a different number of fingers and/or phalanges, and therefore very different in terms of the parameters of the KC, such as the Barrett and IMMA or BruJa hands, obtain a similar degree of anthropomorphism in terms of workspace.

The AIs defined in this study could help to define optimal solutions that maximize the degree of anthropomorphism. Nevertheless, this study has 


\section{4 | Chapter 5}

some limitations. The definition of the AIKCs is based on a comparison with a kinematic model of the $\mathrm{HH}$ that is not perfect because, although it considers all its main DoFs, it assumes universal joint models in the joints with 2 DoFs, which is questionable (Santos and Valero-Cuevas, 2006; Stillfried et al., 2014). Moreover, the assumption that a single kinematic model of the $\mathrm{HH}$ allows representation of the hands of different subjects seems to be dubious (Santos and Valero-Cuevas, 2006). In any case, the model considered is representative of the most advanced and recent biomechanical models of the HH (León et al., 2014; Peña-Pitarch et al., 2014; Sancho-Bru, 2000). Even if new improved hand models are developed in the near future, it would always be possible to maintain, with minor changes, the way in which our indexes are calculated. The mobility range of the different joints of the HH used here could also be revised in the future without major changes in the general methodology. Despite the fact that some recent works have analyzed this last point, they are partial and with little agreement among them (Coupier et al., 2016; Gracia-Ibáñez et al., 2017). In addition, $A I K C_{2}$ and $A I K C_{3}$ could be improved with experimental data measured on the $\mathrm{HH}$, as in (Feix et al., 2013), to obtain a more realistic comparison. Finally, another limitation of the approach followed here is that synergies between the actuation of the various DoFs are not considered in the $\mathrm{HH}$ or in the $\mathrm{AH}$. In fact, in the $\mathrm{HH}$, the mobility ranges of the joints are variable depending on the posture, and in the AHs, which are usually underactuated, each actuator moves more than one DoF, thus affecting the mobility of the different joints. In this sense, we can say that the AIs defined in this study only consider the structure of the $\mathrm{KC}$, but not its relation with the method of actuation. Including the actuation synergies would allow more complete AIs to be defined and is a further development of this study.

\section{Conclusion}

Three alternatives for the definition of the degree of anthropomorphism of the $\mathrm{KC}$ of an $\mathrm{AH}$ have been defined and compared. As a first conclusion, $A I K C_{1}$, based on the comparison of the defining parameters of the $\mathrm{KC}$ (position and orientation vectors, and mobility ranges) and $A I K C_{3}$, based on the comparison of the postures performed during the grasping of characteristic objects, show a high correlation for the hands compared, with the degree of anthropomorphism being around $70-90 \%$ for the five-finger hands and around $40-50 \%$ for the three-finger Barrett hand. As a second conclusion, the AI based on the comparison of the workspace reachable by the joints of the $\mathrm{KC}, A I K C_{2}$, presents much lower values than the previous 
ones (ranging between 1\% and 15\%), and are higher for hands that, like Shadow, combine Ab/Ad and F/E movements in the KC of each finger. As a final conclusion, among the hands compared, the Shadow robotic hand is the one that overall presents the highest values with the defined indexes. The advanced commercial prosthesis i-Limb presents AIKCs comparable to other low-cost AHs. The AIKCs for the three-finger Barrett robotic hand are lower, although the $A I K C_{2}$ value is comparable to that of other five-finger hands.

\section{Acknowledgments}

This work was supported by the Spanish Ministry of Economy and Competitiveness and ESF [grant number BES-2015-076005]; the Spanish Ministry of Economy and Competitiveness, AEI and ERDF [grant numbers DPI2014-60635-R, DPI2017-89910-R]; and Universitat Jaume I, Spain [grant number UJI-B2017-70].

\section{References}

Andrés, F.J., Pérez-González, A., Rubert, C., Fuentes, J., Sospedra, B., 2019. Comparison of Grasping Performance of Tendon and Linkage Transmission Systems in an Electric-Powered Low-Cost Hand Prosthesis. Journal of Mechanisms and Robotics, 11(1), 11018.

Barber, C.B., Dobkin, D.P., Huhdanpaa, H., 1996. The quickhull algorithm for convex hulls. ACM Transactions on Mathematical Software, 22(4), 469-483.

Belter, J.T., Segil, J.L., Dollar, A.M., Weir, R.F., 2013. Mechanical design and performance specifications of anthropomorphic prosthetic hands: A review. The Journal of Rehabilitation Research and Development, 50(5), 599.

Biagiotti, L., Lotti, F., Melchiorri, C., Vassura, G., 2003. How Far Is the Human Hand? A Review on Anthropomorphic Robotic End-effectors, University of Bologna, Italy.

Cobos, S., Ferre, M., Sanchez Uran, M.A., Ortego, J., Pena, C., 2008. Efficient human hand kinematics for manipulation tasks, in: 2008 IEEE/RSJ International Conference on Intelligent Robots and Systems. IEEE, Affiliation: Group of Intellingent Machines, Universidad Politécnica de Madrid, C/José Gutiérrez Abascal, 2, 28006, Madrid, Spain; Correspondence Address: Cobos, S.; Group of Intellingent Machines, Universidad Politécnica de Madrid, C/José Gutiérrez Abasc, pp. 2246-2251.

Coupier, J., Hamoudi, S., Telese-Izzi, S., Feipel, V., Rooze, M., Van Sint Jan, S., 2016. A novel method for in-vivo evaluation of finger kinematics including 
definition of healthy motion patterns. Clinical Biomechanics, 31, 47-58.

Craig, J.J., 2004. Introduction to Robotics: Mechanics and Control 3rd. Prentice Hall, 1(3), 408.

David Gow, n.d. i-Limb. https://www.ossur.com/en-us/prosthetics/arms/i-limbultra

Dermitzakis, K., Ioannides, A., Hwai-ting Lin, 2013. Robotic thumb grasp-based range of motion optimisation, in: 2013 35th Annual International Conference of the IEEE Engineering in Medicine and Biology Society (EMBC). IEEE, pp. 3163-3166.

Duong, T., Wagner, B., Abraham, T., Davidson, M., Bains, G., Daher, N., Friedrich, A., 2017. Comparative Study of Functional Grasp and Efficiency between a 3D-Printed and Commercial Myoelectric Transradial Prosthesis Using Able-Bodied Subjects: A Pilot Study. Journal of Prosthetics and Orthotics, 29(3), 112-118.

Edelsbrunner, H., 2010. Alpha Shapes - a Survey. Tessellations in the Sciences, 125.

Feix, T., Romero, J., Ek, C.H., Schmiedmayer, H.-B., Kragic, D., 2013. A Metric for Comparing the Anthropomorphic Motion Capability of Artificial Hands. IEEE Transactions on Robotics, 29(1), 82-93.

Gibbard, J., 2013. Open Hand Project. www.openhandproject.org

Gibbard, J., Payne, S., 2018. Open Bionics. www.openbionics.com

Gracia-Ibáñez, V., Vergara, M., Sancho-Bru, J.L., Mora, M.C., Piqueras, C., 2017. Functional range of motion of the hand joints in activities of the International Classification of Functioning, Disability and Health. Journal of Hand Therapy, 30(3), 337-347.

Greenhill, R., Elias, H., 2015. Shadow Dexterous Hand. https://www.shadowrobot.com/wp-

content/uploads/shadow_dexterous_hand_technical_specification_E_201508 27.pdf

Hartenberg, R.S., Denavit, J., 1955. A kinematic notation for lower-pair mechanisms based on matrices. Transactions of the ASME. Journal of Applied Mechanics.

Huynh, D.Q., 2009. Metrics for 3D rotations: Comparison and analysis. Journal of Mathematical Imaging and Vision, 35(2), 155-164.

Jiang, L., Liu, Y., Yang, D., Liu, H., 2018. A synthetic framework for evaluating and designing an anthropomorphic prosthetic hand. Journal of Bionic Engineering, 15(1), 69-82.

Kapandji, I.A., 1982. The Physiology of the Joints: Upper limb. Churchill Livingstone. 
Kemp, C., Edsinger, A., Torres-Jara, E., 2007. Challenges for robot manipulation in human environments [Grand Challenges of Robotics]. IEEE Robotics \& Automation Magazine, 14(1), 20-29.

León, B., Morales, A., Sancho-Bru, J.J., León, B., Morales, A., Sancho-Bru, J.J., 2014. From Robot to Human Grasping Simulation, Cognitive Systems Monographs. Springer International Publishing, Cham.

Li, S., Ma, X., Liang, H., Gorner, M., Ruppel, P., Fang, B., Sun, F., Zhang, J., 2019. Vision-based Teleoperation of Shadow Dexterous Hand using End-toEnd Deep Neural Network, in: 2019 International Conference on Robotics and Automation (ICRA). IEEE, pp. 416-422.

Liarokapis, M. V., Artemiadis, P.K., Kyriakopoulos, K.J., 2013. Quantifying anthropomorphism of robot hands, in: 2013 IEEE International Conference on Robotics and Automation. IEEE, pp. 2041-2046.

Liarokapis, M. V., Artemiadis, P.K., Kyriakopoulos, K.J., 2012. Functional Anthropomorphism for human to robot motion mapping, in: Proceedings IEEE International Workshop on Robot and Human Interactive Communication. IEEE, Paris, France, pp. 31-36.

Llop-Harillo, I., Pérez-González, A., 2017. System for the experimental evaluation of anthropomorphic hands. Application to a new 3D-printed prosthetic hand prototype. International Biomechanics, 4(2), 50-59.

Llop-Harillo, I., Pérez-González, A., Gracia-Ibáñez, V., 2019. Anthropomorphism Index of Mobility for Artificial Hands. Applied Bionics and Biomechanics, 2019, 1-11.

OpenAI, Andrychowicz, M., Baker, B., Chociej, M., Józefowicz, R., McGrew, B., Pachocki, J., Petron, A., Plappert, M., Powell, G., Ray, A., Schneider, J., Sidor, S., Tobin, J., Welinder, P., Weng, L., Zaremba, W., 2018. Learning Dexterous In-Hand Manipulation. CoRR.

Parada Puig, J.E., Rodriguez, N.E.N., Ceccarelli, M., 2008. A methodology for the design of robotic hands with multiple fingers. International Journal of Advanced Robotic Systems, 5(2), 177-184.

Peña-Pitarch, E., Falguera, N.T., Yang, J. (James), 2014. Virtual human hand: model and kinematics. Computer Methods in Biomechanics and Biomedical Engineering, 17(5), 568-579.

Sancho-Bru, J.L., 2000. Model biomecànic de la mà orientat al disseny d'eines manuals. Ph.D.

Santos, V.J., Valero-Cuevas, F.J., 2006. Reported anatomical variability naturally leads to multimodal distributions of denavit-hartenberg parameters for the human thumb. IEEE Transactions on Biomedical Engineering, 53(2), 155163.

Simon, A.M., Turner, K.L., Miller, L.A., Hargrove, L.J., Kuiken, T.A., 2019. 
Pattern recognition and direct control home use of a multi-articulating hand prosthesis, in: IEEE International Conference on Rehabilitation Robotics. pp. 386-391.

Soucacos, P.N., 2001. Indications and selection for digital amputation and replantation. Journal of Hand Surgery.

Stillfried, G., Hillenbrand, U., Settles, M., van der Smagt, P., 2014. MRI-Based skeletal hand movement model, in: Springer Tracts in Advanced Robotics. pp. 49-75.

ten Kate, J., Smit, G., Breedveld, P., 2017. 3D-printed upper limb prostheses: a review. Disability and Rehabilitation: Assistive Technology.

Townsend, W., 2013. Barrett Hand. https://advanced.barrett.com/barretthand

Wu, G., Cavanagh, P.R., 1995. ISB recommendations for standardization in the reporting of kinematic data. Journal of Biomechanics, 28(10), 1257-1261.

\section{Supplementary Materials}

The spreadsheet file "DataKinematicChain.xlsx" contains, for the $\mathrm{HH}$ and each $\mathrm{AH}$ analyzed in this study, the elements of the vectors $\boldsymbol{v}_{i, j}$ in Equation 1 that define their KCs. For each segment, the vector of its LCS with respect to its immediate proximal LCS in the chain is shown. Note that $x, y, z$ are non-dimensional values normalized to the hand length (distance from the wrist to the fingertip of the middle finger at the RP), and $\theta_{x}, \theta_{y}, \theta_{z}$ are the Euler angles around the $\mathrm{X}, \mathrm{Y}$ and $\mathrm{Z}$ axes respectively, with order of rotations XZY, expressed in radians. For the digits with four segments, the row labeled medial phalanx actually corresponds to the distal one and the row labeled distal phalanx corresponds to the fingertip of the digit. The spreadsheet also contains the minimum and maximum range of mobility of each joint around the reference posture for the $\mathrm{HH}$ and each $\mathrm{AH}$ analyzed.

The document "GraspingPostures.pdf" contains the optimization functions $f_{k}$ used to obtain the grasping postures of the different objects and all their geometrical dimensions and their positions for computing $\mathrm{AIKC}_{3}$.

Data Kinematic Chain:

\begin{tabular}{llccccc}
\hline Human Hand & $\boldsymbol{v}_{\mathbf{i} . \mathbf{j}}$ & Thumb & Index & Middle & Ring & Little \\
\hline \multirow{4}{*}{ Metacarpal } & $x$ & 0 & 0 & 0 & 0 & 0 \\
& $y$ & 0.073 & 0.073 & 0.073 & 0.073 & 0.073 \\
& $z$ & 0.0842 & 0.0541 & 0 & -0.0554 & -0.0958 \\
& $\theta_{x}$ & 0.5686 & 0.1438 & 0 & -0.0981 & -0.2427 \\
& $\theta_{y}$ & -1.5355 & 0 & 0 & 0 & 0 \\
& $\theta_{z}$ & 0.4044 & 0 & 0 & 0 & 0 \\
\hline
\end{tabular}




\begin{tabular}{ccccccc} 
& $x$ & 0 & 0 & 0 & 0 & 0 \\
& $y$ & 0.251 & 0.3779 & 0.373 & 0.3376 & 0.3049 \\
Proximal phalanx & $z$ & 0 & 0 & 0 & 0 & 0 \\
& $\theta_{x}$ & 0 & -0.2618 & -0.1047 & 0 & 0.1222 \\
& $\theta_{y}$ & 0 & 0 & 0 & 0 & 0 \\
& $\theta_{z}$ & 0 & 0 & 0 & 0 & 0 \\
\hline \multirow{5}{*}{ Medial phalanx } & $x$ & 0 & 0 & 0 & 0 & 0 \\
& $y$ & 0.196 & 0.2649 & 0.2773 & 0.2591 & 0.2059 \\
& $z$ & 0 & 0 & 0 & 0 & 0 \\
& $\theta_{x}$ & 0 & 0 & 0 & 0 & 0 \\
& $\theta_{y}$ & 0 & 0 & 0 & 0 & 0 \\
& $\theta_{z}$ & 0 & 0 & 0 & 0 & 0 \\
\hline \multirow{5}{*}{ Distal phalanx } & $x$ & 0 & 0 & 0 & 0 & 0 \\
& $y$ & 0.158 & 0.143 & 0.17 & 0.165 & 0.117 \\
& $z$ & 0 & 0 & 0 & 0 & 0 \\
& $\theta_{x}$ & 0 & 0 & 0 & 0 & 0 \\
& $\theta_{y}$ & 0 & 0 & 0 & 0 & 0 \\
& $\theta_{z}$ & 0 & 0 & 0 & 0 & 0 \\
\hline \multirow{6}{*}{ Fingertip } & $x$ & 0 & 0 & 0 & 0 & 0 \\
& $y$ & 0 & 0.097 & 0.108 & 0.107 & 0.093 \\
& $z$ & 0 & 0 & 0 & 0 & 0 \\
& $\theta_{x}$ & 0 & 0 & 0 & 0 & 0 \\
& $\theta_{y}$ & 0 & 0 & 0 & 0 & 0 \\
& $\theta_{z}$ & 0 & 0 & 0 & 0 & 0 \\
\hline \multirow{6}{*}{} & & & & & &
\end{tabular}

\begin{tabular}{llccccc}
\hline Human Hand & & Thumb & Index & Middle & Ring & Little \\
\hline \multirow{5}{*}{ Minimum angle $\left(^{\circ}\right.$ ) } & CMC add & -30 & 0 & 0 & 0 & 0 \\
& CMC ext & -25 & 0 & 0 & 0 & 0 \\
& MCP add & -30 & -15 & -8 & -20 & -40 \\
& MCP ext & -10 & 0 & 0 & 0 & 0 \\
& PIP ext & -15 & 0 & 0 & 0 & 0 \\
& DIP ext & 0 & -10 & -10 & -20 & -30 \\
\hline \multirow{5}{*}{ Maximum angle $\left({ }^{\circ}\right)$} & CMC abd & 60 & 0 & 0 & 0 & 0 \\
& CMC flex & 35 & 0 & 0 & 15 & 30 \\
& MCP abd & 60 & 42 & 35 & 14 & 19 \\
& MCP flex & 80 & 90 & 90 & 90 & 90 \\
& PIP flex & 80 & 100 & 100 & 100 & 100 \\
& DIP flex & 0 & 90 & 90 & 90 & 90 \\
\hline
\end{tabular}

\begin{tabular}{llccc}
\hline Barrett & $\boldsymbol{v}_{\mathbf{i} . \mathbf{j}}$ & Thumb & Index & Middle \\
\hline \multirow{4}{*}{ Metacarpal } & $x$ & 0 & 0 & 0 \\
& $y$ & 0 & 0 & 0 \\
& $z$ & 0 & 0.1493 & -0.1493 \\
& $\theta_{x}$ & 3.1416 & 0 & 0 \\
& $\theta_{y}$ & 0 & 0 & 0 \\
& $\theta_{z}$ & 0 & 0 & 0 \\
\hline \multirow{5}{*}{ Proximal phalanx } & $x$ & 0 & 0 & 0 \\
& $y$ & 0.2985 & 0.2985 & 0.2985 \\
& $z$ & 0 & 0 & 0 \\
& $\theta_{x}$ & 0 & 0 & 0 \\
\multirow{2}{*}{ Medial phalanx } & $\theta_{y}$ & 0 & 0 & 0 \\
& $\theta_{z}$ & 0 & 0 & 0 \\
\hline & $x$ & -0.0179 & -0.0239 & -0.0239 \\
& $y$ & 0.4179 & 0.4179 & 0.4179
\end{tabular}




\begin{tabular}{ccccc} 
& $z$ & 0 & 0 & 0 \\
& $\theta_{x}$ & 0 & 0 & 0 \\
& $\theta_{y}$ & 0 & 0 & 0 \\
& $\theta_{z}$ & 0.6912 & 0.6912 & 0.6912 \\
\hline \multirow{5}{*}{ Distal phalanx } & $x$ & 0 & 0 & 0 \\
& $y$ & 0.3463 & 0.3463 & 0.3463 \\
& $z$ & 0 & 0 & 0 \\
& $\theta_{x}$ & 0 & 0 & 0 \\
& $\theta_{y}$ & 0 & 0 & 0 \\
& $\theta_{z}$ & 0 & 0 & 0 \\
\hline \multirow{5}{*}{ Fingertip } & $x$ & 0 & 0 & 0 \\
& $y$ & 0 & 0 & 0 \\
& $z$ & 0 & 0 & 0 \\
& $\theta_{x}$ & 0 & 0 & 0 \\
& $\theta_{y}$ & 0 & 0 & 0 \\
& $\theta_{z}$ & 0 & 0 & 0 \\
\hline
\end{tabular}

\begin{tabular}{llccccc}
\hline Barrett & & Thumb & Index & Middle & Ring & Little \\
\hline \multirow{5}{*}{ Minimum angle $\left(^{\circ}\right.$ ) } & CMC add & 0 & 0 & -180 & 0 & 0 \\
& CMC ext & 0 & 0 & 0 & 0 & 0 \\
& MCP add & 0 & 0 & 0 & 0 & 0 \\
& MCP ext & 0 & 0 & 0 & 0 & 0 \\
& PIP ext & 0 & 0 & 0 & 0 & 0 \\
& DIP ext & 0 & 0 & 0 & 0 & 0 \\
\hline \multirow{5}{*}{ Maximum angle $\left(^{\circ}\right)$} & CMC abd & 0 & 180 & 0 & 0 & 0 \\
& CMC flex & 0 & 0 & 0 & 0 & 0 \\
& MCP abd & 0 & 0 & 0 & 0 & 0 \\
& MCP flex & 140 & 140 & 140 & 0 & 0 \\
& PIP flex & 45 & 45 & 45 & 0 & 0 \\
& DIP flex & 0 & 0 & 0 & 0 & 0 \\
\hline
\end{tabular}

\begin{tabular}{|c|c|c|c|c|c|c|}
\hline BruJa & $v_{i . j}$ & Thumb & Index & Middle & Ring & Little \\
\hline \multirow{6}{*}{ Metacarpal } & $x$ & 0.01 & 0 & 0 & 0 & 0 \\
\hline & $y$ & 0.1775 & 0 & 0 & 0 & 0 \\
\hline & $Z$ & 0.1271 & 0 & 0 & 0 & 0 \\
\hline & $\theta_{x}$ & 1.5708 & 0 & 0 & 0 & 0 \\
\hline & $\theta_{y}$ & 0 & 0 & 0 & 0 & 0 \\
\hline & $\theta_{z}$ & 0 & 0 & 0 & 0 & 0 \\
\hline \multirow{6}{*}{ Proximal phalanx } & $x$ & 0.0215 & 0.0412 & 0.0412 & 0.0412 & 0.0412 \\
\hline & $y$ & 0.314 & 0.5673 & 0.5673 & 0.5673 & 0.5673 \\
\hline & $z$ & -0.125 & 0.1817 & 0.0718 & -0.0412 & -0.1483 \\
\hline & $\theta_{x}$ & -1.1847 & 0 & 0 & 0 & 0 \\
\hline & $\theta_{y}$ & -1.5708 & 0 & 0 & 0 & 0 \\
\hline & $\theta_{z}$ & 0 & 0 & 0 & 0 & 0 \\
\hline \multirow{6}{*}{ Medial phalanx } & $x$ & 0 & 0 & 0 & 0 & 0 \\
\hline & $y$ & 0.1581 & 0.186 & 0.2093 & 0.1953 & 0.1395 \\
\hline & $z$ & 0 & 0 & 0 & 0 & 0 \\
\hline & $\theta_{x}$ & 0 & 0 & 0 & 0 & 0 \\
\hline & $\theta_{y}$ & 0 & 0 & 0 & 0 & 0 \\
\hline & $\theta_{z}$ & 0 & 0 & 0 & 0 & 0 \\
\hline \multirow{4}{*}{ Distal phalanx } & $x$ & 0 & 0 & 0 & 0 & 0 \\
\hline & $y$ & 0.1349 & 0.093 & 0.1163 & 0.1023 & 0.0651 \\
\hline & $Z$ & 0 & 0 & 0 & 0 & 0 \\
\hline & $\theta_{x}$ & 0 & 0 & 0 & 0 & 0 \\
\hline
\end{tabular}




\begin{tabular}{ccccccc} 
& $\theta_{y}$ & 0 & 0 & 0 & 0 & 0 \\
\hline \multirow{4}{*}{ Fingertip } & $\theta_{z}$ & 0 & 0 & 0 & 0 & 0 \\
\hline \multirow{6}{*}{} & $x$ & 0 & 0 & 0 & 0 & 0 \\
& $y$ & 0 & 0.1023 & 0.1116 & 0.107 & 0.093 \\
& $z$ & 0 & 0 & 0 & 0 & 0 \\
& $\theta_{x}$ & 0 & 0 & 0 & 0 & 0 \\
& $\theta_{y}$ & 0 & 0 & 0 & 0 & 0 \\
$\theta_{z}$ & 0 & 0 & 0 & 0 & 0 \\
\hline
\end{tabular}

\begin{tabular}{llccccc}
\hline BruJa & & Thumb & Index & Middle & Ring & Little \\
\hline \multirow{5}{*}{ Minimum angle $\left({ }^{\circ}\right.$ ) } & CMC add & 0 & 0 & 0 & 0 & 0 \\
& CMC ext & 0 & 0 & 0 & 0 & 0 \\
& MCP add & -1 & -1 & -1 & -1 & -1 \\
& MCP ext & 0 & 0 & 0 & 0 & 0 \\
& PIP ext & 5 & 5 & 5 & 5 & 5 \\
& DIP ext & 0 & 5 & 5 & 5 & 5 \\
\hline \multirow{5}{*}{ Maximum angle $\left({ }^{\circ}\right)$} & CMC abd & 0 & 0 & 0 & 0 & 0 \\
& CMC flex & 90 & 0 & 0 & 0 & 0 \\
& MCP abd & 1 & 1 & 1 & 1 & 1 \\
& MCP flex & 42 & 90 & 90 & 90 & 90 \\
& PIP flex & 70 & 77 & 69 & 67 & 60 \\
& DIP flex & 0 & 51 & 62 & 65 & 55 \\
\hline
\end{tabular}

\begin{tabular}{|c|c|c|c|c|c|c|}
\hline IMMA & $v_{i . j}$ & Thumb & Index & Middle & Ring & Little \\
\hline \multirow{6}{*}{ Metacarpal } & $x$ & 0 & 0 & 0 & 0 & 0 \\
\hline & $y$ & 0.2169 & 0 & 0 & 0 & 0 \\
\hline & $z$ & 0.1577 & 0 & 0 & 0 & 0 \\
\hline & $\theta_{x}$ & 1.5708 & 0 & 0 & 0 & 0 \\
\hline & $\theta_{y}$ & 0 & 0 & 0 & 0 & 0 \\
\hline & $\theta_{z}$ & 0 & 0 & 0 & 0 & 0 \\
\hline \multirow{6}{*}{ Proximal phalanx } & $x$ & 0 & 0 & 0 & 0 & 0 \\
\hline & $y$ & 0.132 & 0.4588 & 0.437 & 0.4192 & 0.3874 \\
\hline & $z$ & 0 & 0.1188 & 0.0016 & -0.1054 & -0.2004 \\
\hline & $\theta_{x}$ & 0 & 0.0873 & 0 & -0.125 & -0.2618 \\
\hline & $\theta_{y}$ & -0.7854 & 0 & 0 & 0 & 0 \\
\hline & $\theta_{z}$ & 0 & 0 & 0 & 0 & 0 \\
\hline \multirow{6}{*}{ Medial phalanx } & $x$ & 0 & 0 & 0 & 0 & 0 \\
\hline & $y$ & 0.2158 & 0.2456 & 0.2725 & 0.2291 & 0.1968 \\
\hline & $z$ & 0 & 0 & 0 & 0 & 0 \\
\hline & $\theta_{x}$ & 0 & 0 & 0 & 0 & 0 \\
\hline & $\theta_{y}$ & 0 & 0 & 0 & 0 & 0 \\
\hline & $\theta_{z}$ & 0 & 0 & 0 & 0 & 0 \\
\hline \multirow{6}{*}{ Distal phalanx } & $x$ & 0 & 0 & 0 & 0 & 0 \\
\hline & $y$ & 0.1659 & 0.1399 & 0.1623 & 0.1509 & 0.059 \\
\hline & $z$ & 0 & 0 & 0 & 0 & 0 \\
\hline & $\theta_{x}$ & 0 & 0 & 0 & 0 & 0 \\
\hline & $\theta_{y}$ & 0 & 0 & 0 & 0 & 0 \\
\hline & $\theta_{z}$ & 0 & 0 & 0 & 0 & 0 \\
\hline \multirow{6}{*}{ Fingertip } & $x$ & 0 & 0 & 0 & 0 & 0 \\
\hline & $y$ & 0 & 0.1243 & 0.1324 & 0.1324 & 0.1135 \\
\hline & $z$ & 0 & 0 & 0 & 0 & 0 \\
\hline & $\theta_{x}$ & 0 & 0 & 0 & 0 & 0 \\
\hline & $\theta_{y}$ & 0 & 0 & 0 & 0 & 0 \\
\hline & $\theta_{z}$ & 0 & 0 & 0 & 0 & 0 \\
\hline
\end{tabular}




\begin{tabular}{llccccc}
\hline IMMA & & Thumb & Index & Middle & Ring & Little \\
\hline \multirow{5}{*}{ Minimum angle $\left({ }^{\circ}\right)$} & CMC add & 0 & 0 & 0 & 0 & 0 \\
& CMC ext & -10 & 0 & 0 & 0 & 0 \\
& MCP add & -1 & -1 & -1 & -1 & -1 \\
& MCP ext & -11 & -20 & -17 & -15 & -20 \\
& PIP ext & -13 & -20 & -17 & -20 & -20 \\
& DIP ext & 0 & -15 & -20 & -20 & -20 \\
\hline \multirow{5}{*}{ Maximum angle $\left(^{\circ}\right)$ ) } & CMC abd & 0 & 0 & 0 & 0 & 0 \\
& CMC flex & 70 & 0 & 0 & 0 & 0 \\
& MCP flex & 55 & 85 & 86.3 & 70 & 65 \\
& PIP flex & 55 & 60 & 75 & 75 & 65 \\
& DIP flex & 0 & 50 & 75 & 70 & 75 \\
\hline
\end{tabular}

\begin{tabular}{|c|c|c|c|c|c|c|}
\hline Shadow & $v_{i . j}$ & Thumb & Index & Middle & Ring & Little \\
\hline \multirow{6}{*}{ Metacarpal } & $x$ & -0.0436 & 0 & 0 & 0 & 0 \\
\hline & $y$ & 0.1487 & 0 & 0 & 0 & 0.1538 \\
\hline & $Z$ & 0.1744 & 0 & 0 & 0 & -0.1231 \\
\hline & $\theta_{x}$ & 0.7854 & 0 & 0 & 0 & -0.9599 \\
\hline & $\theta_{y}$ & 0 & 0 & 0 & 0 & 0 \\
\hline & $\theta_{z}$ & 0 & 0 & 0 & 0 & 0 \\
\hline \multirow{6}{*}{ Proximal phalanx } & $x$ & 0 & 0 & 0 & 0 & 0 \\
\hline & $y$ & 0.1949 & 0.4872 & 0.5077 & 0.4872 & 0.2043 \\
\hline & $z$ & 0 & 0.1744 & 0.0564 & -0.0564 & 0.2113 \\
\hline & $\theta_{x}$ & 0 & 0 & 0 & 0 & 0.9599 \\
\hline & $\theta_{y}$ & -1.5708 & 0 & 0 & 0 & 0 \\
\hline & $\theta_{z}$ & 0 & 0 & 0 & 0 & 0 \\
\hline \multirow{6}{*}{ Medial phalanx } & $x$ & 0 & 0 & 0 & 0 & 0 \\
\hline & $y$ & 0.1641 & 0.2308 & 0.2308 & 0.2308 & 0.2308 \\
\hline & $z$ & 0 & 0 & 0 & 0 & 0 \\
\hline & $\theta_{x}$ & 0 & 0 & 0 & 0 & 0 \\
\hline & $\theta_{y}$ & 0 & 0 & 0 & 0 & 0 \\
\hline & $\theta_{z}$ & 0 & 0 & 0 & 0 & 0 \\
\hline \multirow{6}{*}{ Distal phalanx } & $x$ & 0 & 0 & 0 & 0 & 0 \\
\hline & $y$ & 0.141 & 0.1282 & 0.1282 & 0.1282 & 0.1282 \\
\hline & $z$ & 0 & 0 & 0 & 0 & 0 \\
\hline & $\theta_{x}$ & 0 & 0 & 0 & 0 & 0 \\
\hline & $\theta_{y}$ & 0 & 0 & 0 & 0 & 0 \\
\hline & $\theta_{z}$ & 0 & 0 & 0 & 0 & 0 \\
\hline \multirow{6}{*}{ Fingertip } & $x$ & 0 & 0 & 0 & 0 & 0 \\
\hline & $y$ & 0 & 0.1333 & 0.1333 & 0.1333 & 0.1333 \\
\hline & $Z$ & 0 & 0 & 0 & 0 & 0 \\
\hline & $\theta_{x}$ & 0 & 0 & 0 & 0 & 0 \\
\hline & $\theta_{y}$ & 0 & 0 & 0 & 0 & 0 \\
\hline & $\theta_{z}$ & 0 & 0 & 0 & 0 & 0 \\
\hline
\end{tabular}

\begin{tabular}{llccccc}
\hline Shadow & & Thumb & Index & Middle & Ring & Little \\
\hline \multirow{5}{*}{ Minimum angle $\left(^{\circ}\right)$} & CMC add & -60 & 0 & 0 & 0 & 0 \\
& CMC ext & 0 & 0 & 0 & 0 & 0 \\
& MCP add & -30 & -20 & -20 & -20 & -20 \\
& MCP ext & -12 & 0 & 0 & 0 & 0 \\
& PIP ext & 0 & 0 & 0 & 0 & 0 \\
& DIP ext & 0 & 0 & 0 & 0 & 0 \\
\hline
\end{tabular}




$\begin{array}{clccccc} & \text { CMC abd } & 60 & 0 & 0 & 0 & 0 \\ \text { Maximum angle }\left({ }^{\circ}\right) & \text { CMC flex } & 70 & 0 & 0 & 0 & 45 \\ & \text { MCP abd } & 30 & 20 & 20 & 20 & 20 \\ & \text { MCP flex } & 12 & 90 & 90 & 90 & 90 \\ & \text { PIP flex } & 90 & 90 & 90 & 90 & 90 \\ & \text { DIP flex } & 0 & 90 & 90 & 90 & 90\end{array}$

*The CMC add/abd DoF of the thumb actually corresponds to an external/internal rotation around the $\mathrm{Y}$ axis

\begin{tabular}{|c|c|c|c|c|c|c|}
\hline i-Limb & $v_{\text {i.j }}$ & Thumb & Index & Middle & Ring & Little \\
\hline \multirow{6}{*}{ Metacarpal } & $x$ & -0.0666 & 0 & 0 & 0 & 0 \\
\hline & $y$ & 0.2164 & 0 & 0 & 0 & 0 \\
\hline & $Z$ & 0.0971 & 0 & 0 & 0 & 0 \\
\hline & $\theta_{x}$ & 1.5708 & 0 & 0 & 0 & 0 \\
\hline & $\theta_{y}$ & 0 & 0 & 0 & 0 & 0 \\
\hline & $\theta_{z}$ & 0 & 0 & 0 & 0 & 0 \\
\hline \multirow{6}{*}{ Proximal phalanx } & $x$ & 0 & 0.0633 & 0.0633 & 0.0633 & 0.0633 \\
\hline & $y$ & 0.1387 & 0.5316 & 0.5339 & 0.505 & 0.4856 \\
\hline & $Z$ & 0.0555 & 0.157 & 0.0555 & -0.0555 & -0.1609 \\
\hline & $\theta_{x}$ & -1.1868 & 0 & 0 & 0 & 0 \\
\hline & $\theta_{y}$ & -1.5708 & 0 & 0 & 0 & 0 \\
\hline & $\theta_{z}$ & 0 & 0.4363 & 0.4363 & 0.4363 & 0.4363 \\
\hline \multirow{6}{*}{ Medial phalanx } & $x$ & 0 & 0 & 0 & 0 & 0 \\
\hline & $y$ & 0.3147 & 0.2497 & 0.2442 & 0.2403 & 0.2081 \\
\hline & $Z$ & 0 & 0 & 0 & 0 & 0 \\
\hline & $\theta_{x}$ & 0 & 0 & 0 & 0 & 0 \\
\hline & $\theta_{y}$ & 0 & 0 & 0 & 0 & 0 \\
\hline & $\theta_{z}$ & 0 & 0 & 0 & 0 & 0 \\
\hline \multirow{6}{*}{ Distal phalanx } & $x$ & 0 & 0 & 0 & 0 & 0 \\
\hline & $y$ & 0.1665 & 0.1343 & 0.1387 & 0.1387 & 0.1249 \\
\hline & $Z$ & 0 & 0 & 0 & 0 & 0 \\
\hline & $\theta_{x}$ & 0 & 0 & 0 & 0 & 0 \\
\hline & $\theta_{y}$ & 0 & 0 & 0 & 0 & 0 \\
\hline & $\theta_{z}$ & 0 & 0.1396 & 0.1396 & 0.1396 & 0.1396 \\
\hline \multirow{6}{*}{ Fingertip } & $x$ & 0 & 0 & 0 & 0 & 0 \\
\hline & $y$ & 0 & 0.0694 & 0.0832 & 0.0832 & 0.0555 \\
\hline & $Z$ & 0 & 0 & 0 & 0 & 0 \\
\hline & $\theta_{x}$ & 0 & 0 & 0 & 0 & 0 \\
\hline & $\theta_{y}$ & 0 & 0 & 0 & 0 & 0 \\
\hline & $\theta_{z}$ & 0 & 0 & 0 & 0 & 0 \\
\hline
\end{tabular}

\begin{tabular}{llccccc}
\hline i-Limb & & Thumb & Index & Middle & Ring & Little \\
\hline \multirow{5}{*}{ Minimum angle $\left(^{\circ}{ }^{\circ}\right)$} & CMC add & 0 & 0 & 0 & 0 & 0 \\
& CMC ext & 0 & 0 & 0 & 0 & 0 \\
& MCP add & -1 & -1 & -1 & -1 & -1 \\
& MCP ext & -10 & -1 & -1 & -1 & -1 \\
& PIP ext & 0 & 0 & 0 & 0 & 0 \\
& DIP ext & 0 & 0 & 0 & 0 & 0 \\
\hline \multirow{5}{*}{ Maximum angle $\left(^{\circ}\right.$ ) } & CMC abd & 0 & 0 & 0 & 0 & 0 \\
& CMC flex & 90 & 0 & 0 & 0 & 0 \\
& MCP abd & 1 & 1 & 1 & 1 & 1 \\
& MCP flex & 50 & 90 & 90 & 90 & 90 \\
& PIP flex & 90 & 90 & 90 & 90 & 90 \\
& DIP flex & 0 & 0 & 0 & 0 & 0 \\
\hline
\end{tabular}




\section{4 | Chapter 5}

Grasping Postures:

\section{Optimization functions for grasping postures}

\section{Spherical object}

For the spherical object $(k=1)$, the optimization function $f_{k}$ used to obtain the grasping posture was:

$$
f_{1}=\sum_{i j}\left(R-\left\|\boldsymbol{r}_{\boldsymbol{i j}}-\boldsymbol{p}_{\boldsymbol{c}}\right\|\right) \quad i, j=1, \ldots 5
$$

where $\boldsymbol{p}_{\boldsymbol{c}}$ is the position vector of the center of the sphere with respect to $\left\{\mathrm{LCS}_{0}\right\}, R$ is its radius, and $\boldsymbol{r}_{i j}$ is the position vector of $L C S_{i, j}$, located at joint $j$ of digit $i$. The definition of $f_{1}$ comprises the sum of all the distances to be minimized, namely that the distance of each LCS to the center of the sphere approximates $R$, except for those corresponding to the CMC joints.

\section{Prismatic object}

For the prismatic object $(k=2)$, the two main dimensions were placed on a plane parallel to ZY (see Fig. 3). The optimization function $f_{2}$ used was:

$$
f_{2}=\sum_{i}\left|x_{f}-x_{i}\right|+\sum_{j}\left|x_{t}-x_{j}\right|
$$

The first summation of Eq. (A2) evaluates and accumulates the distances of all the LCSs of the long fingers, except those corresponding to the CMC joints, to the prism face on which the long fingers make contact, at $x_{f}$. Likewise, the second summation evaluates and accumulates the distances of the LCSs of the thumb, except for the CMC joint, up to the opposed prism face, at $x_{t}$. The function to be minimized, $f_{2}$, resumes both evaluations.

\section{Cylindrical object}

The cylindrical object ( $k=3$ ) was placed parallel to the $Z$ axis (see Fig. 3). The optimization function used for $f_{3}$ was:

$$
f_{3}=\sum_{i j}\left|R-d_{i j}\right|
$$

where $R$ is the radius of the cylinder and $d_{i j}$ is the distance from the $L C S_{i, j}$ of the hand to the axis of the cylinder, extending the summation to all the LCSs of the hand except those corresponding to the CMC joints.

\section{Object dimensions}

All the geometrical dimensions used to define the objects and their positions were normalized by dividing by the hand length (distance from the 
wrist to the end of the middle finger) and were expressed in the fixed $\left\{\mathrm{LCS}_{0}\right\}$ of the wrist:

- Spherical object: $R=0.3, \boldsymbol{p}_{\boldsymbol{c}}=[-0.3,0.3,0]$

- Prismatic object: $x_{f}=-0.2, x_{t}=0$

- Cylindrical object: $R=0.2$, center coordinates $\boldsymbol{p}_{\boldsymbol{c}}=[-0.2,0.5,0]$, axis parallel to Z-axis 



\section{Chapter 6}

Optimization of the Kinematic

Chain of the Thumb for a Hand Prosthesis Based on the Kapandji Opposition Test 

This chapter corresponds exactly to the published book chapter:

Optimization of the Kinematic Chain of the Thumb for a Hand Prosthesis Based on the Kapandji Opposition Test Antonio Pérez-González and Immaculada Llop-Harillo In: Computer Methods, Imaging and Visualization in Biomechanics and Biomedical Engineering, Springer, 2020, 36, 271-287

\begin{abstract}
The thumb plays a key role in the performance of the hand for grasping and manipulating objects. In artificial hands the complex thumb's kinematic chain (TKC) is simplified and its five degrees of freedom are reduced to only one or two with the consequent loss of dexterity of the hand. The Kapandji opposition test (KOT) has been clinically used in pathological human hands for evaluating the thumb opposition and it has also been employed in some previous studies as reference for the design of the TKC in artificial hands, but without a clearly stated methodology. Based on this approaches, in this study we present a computational method to optimize the whole TKC (base placement, link lengths and joint orientation angles) of an artificial hand based on its performance in the KOT. The cost function defined for the optimization (MPE) is a weighted mean position error when trying to reproduce the KOT postures and can be used also as a metric to quantify thumb opposition in the hand. As a case study, the method was applied to the improvement of the TKC of an artificial hand developed by the authors and the MPE was reduced to near one third of that of the original design, increasing significantly the number of reachable positions in the KOT. The metric proposed based on the KOT can be used directly or in combination with other to improve the kinematic chain of artificial hands.
\end{abstract}

\title{
Keywords
}

Artificial hand, kinematic chain, optimization

\section{Introduction}

The human hand is a marvelous tool optimized in an evolutionary process since our ancestors (Bardo et al., 2018; Feix et al., 2015). Thumb opposition is said to be one distinctive feature of the human hand. Interestingly, this 


\section{0 | Chapter 6}

dexterity can be obtained even with an important variability in the thumb anatomy among individuals (Santos and Valero-cuevas, 2006). The human thumb is composed of three bones (Figure 1) (Giurintano et al., 1995): the distal phalanx, the proximal phalanx and the first metacarpal bone, connected to the wrist. The interphalangeal joint (IP) is a hinge joint with one degree of freedom (DoF) whereas the metacarpo-phalangeal joint (MCP) is condylar and the carpo-metacarpal joint (CMC) is of saddle type, both with two DoFs. Therefore, the thumb's kinematic chain (TKC) can be considered as an open chain connected to the wrist with 5 DoFs, allowing a high range of positions and orientations of the thumb tip. It has been shown that the assumption of universal joints (two perpendicular and intersecting axes of rotation) for the CMC and MCP joints is not realistic and that a biomechanical model with five links (Giurintano et al., 1995), including two virtual links in these joints and considering non-orthogonal and non-intersecting axes in the joints is more realistic and represents better the anatomical evidences (Santos and Valero-cuevas, 2006).

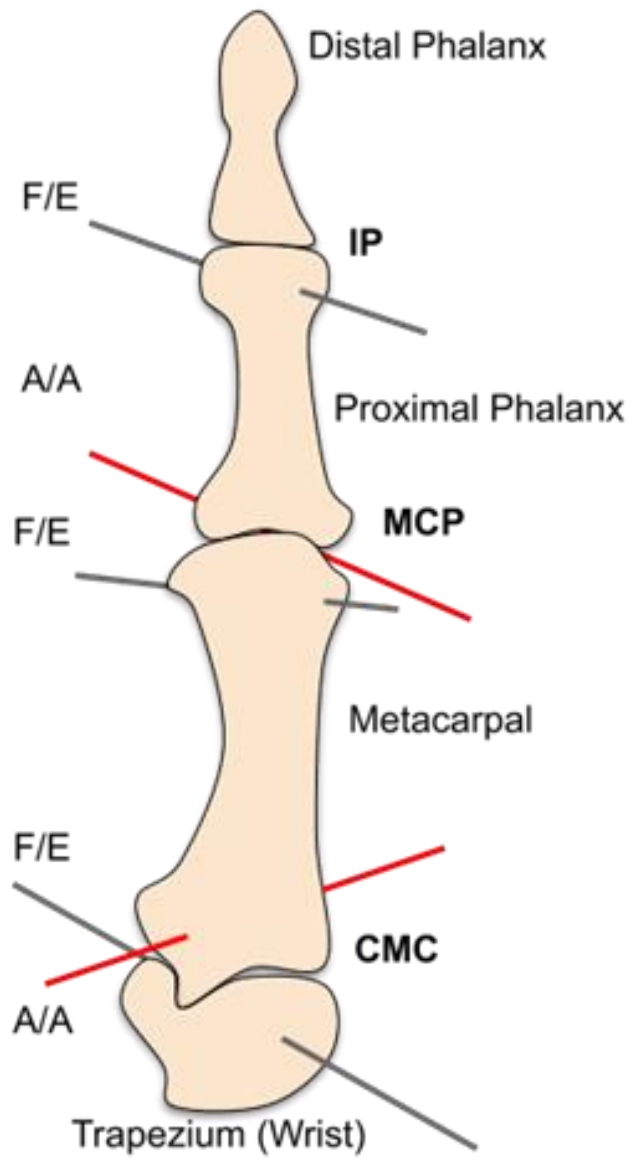

Figure 1. Bones and joints of the human thumb (Giurintano et al., 1995). 
The design of artificial hands, both prosthetic and robotic, is moving in last decades towards devices more anthropomorphic, to improve the functionality and the cosmetic appearance. Given the complexity of the TKC above explained, the designers of artificial hands need to introduce simplifications for this digit. These simplifications are mainly guided by the difficulty of obtaining adequate mechanical solutions for reproducing the geometry and mobility of the CMC and MCP joints, but also by the attempt to simplify the artificial hand control. Generally, the five DoFs of the human thumb are simplified in mechanical hands to achieve two basic motions: flexion/extension and circumduction. The circumduction rotation of the thumb is the movement requested to change the type of opposition of the thumb with respect to the long fingers, it allows to alternate between a lateral grasp and a power or precision grasp. In the human hand, the circumduction motion is achieved through a combination of 3 joints at the base of the thumb (Coert et al., 2003). Belter et al. (Belter et al., 2013) reviewed the thumb design and position for different prosthetic hands. They highlighted the relevance of the relationship between the circumduction rotation axis of the thumb and the main axis of the wrist for functional grasps. In most of the prosthetic hands that Belter et al. analyzed, the thumb is actuated with a simple closing or opening (flexion/extension) and along the circumduction rotation axis, that is not always oriented parallel with the wrist rotation axis. They recommended to jointly approximate in a single DoF the thumb flexion and circumduction rotation for keeping complexity low. Ten Kate et al. (ten Kate et al., 2017) reviewed the kinematic specifications of 3D-printed hand prostheses and specified the range of motion for the thumb flexion and thumb circumduction of 58 devices. Three of the hands analyzed lack a thumb, 7\% of the thumbs of the other hands did not perform flexion movement and 62\% did not perform circumduction movement. Grebenstein et al. (Grebenstein et al., 2010) analyzed anatomy, surgery and rehabilitation data for defining some guidelines to be used for the design of a robotic thumb for the DLR hand. They proposed a minimum of 3 DoF to allow proper manipulation.

From the literature of both robotic and prosthetic fields, we can find thumbs with different mechanical configurations, changing the number of links and DoFs. Figure 2 shows several representative examples.

The thumb of the SensorHand Speed (Ottobock, 2017) (Figure 2a) is a rigid bar with only 1 DoF. The thumb of the FRH-4 Hand of the mobileassisting robot ARMAR (Gaiser et al., 2008) (Figure 2b) has 2 DoFs actuated by two independent fluidic actuators that produce flexion motion of the MCP 


\section{2 | Chapter 6}

and IP joints, respectively. The thumb of the Bebionic hand (Ottobock, 2017) (Figure 2c) has 3 DoFs, one actuator produce the flexion of the MCP and IP joints and the MCP circumduction has two selectable fixed positions, manually placed by the user of the prosthesis. The thumb of the DLR/HIT Hand II (Liu et al., 2008) (Figure 2d) has 4 DoFs and 3 actuators, one for the CMC flexion, other for the MCP and IP flexion and other for the CMC abduction. The thumb of the Shadow Dexterous Hand (Shadow Robot Company, 2019) (Figure 2e) has 5 independently actuated DoFs, as the human hand, but the MCP and CMC are universal joints.

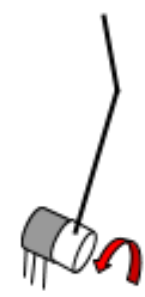

(a)

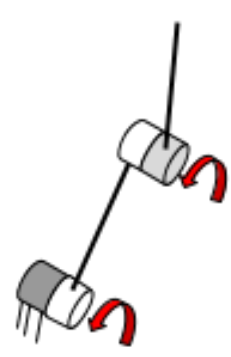

(b)

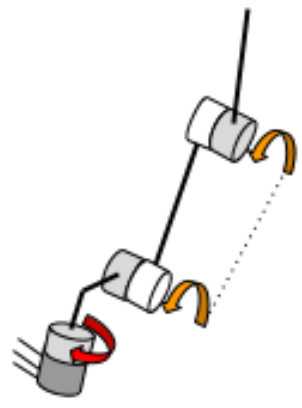

(c)

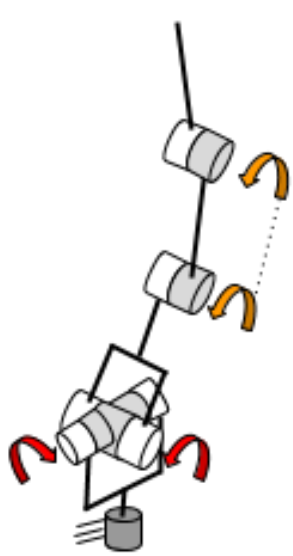

(d)

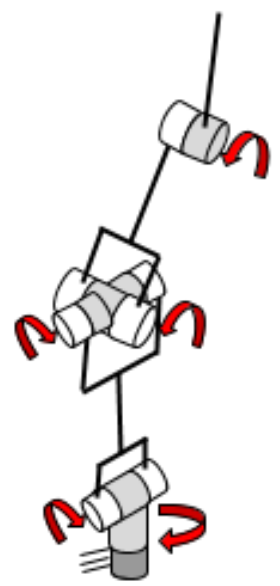

(e)

Figure 2. Thumb's kinematic chain (TKC) for several artificial hands. a: SensorHand Speed (Ottobock, 2017), b: FRH-4 Hand (Gaiser et al., 2008), c:

Bebionic hand (Ottobock, 2017), d: DLR/HIT Hand II (Liu et al., 2008), e: Shadow Dexterous Hand (Shadow Robot Company, 2019). Red arrow: actuated by an independent actuator; yellow arrow: several DoFs actuated by the same actuator.

It could be interesting to have objective methods to evaluate the impact of simplifications made in the thumb of artificial hands in the loss of ability to grasp in real life applications. These objective methods could help designers to obtain hand designs with improved grasping abilities. The Kapandji opposition test (KOT) (Kapandji, 1986), also called total opposition test, can be of interest for this goal. The KOT was proposed as a simple method for assessing the opposition of the thumb in pathological hands and is used in current clinical practice. It involves touching different areas of the four long fingers with the tip of the thumb (Figure 3). The score obtained in the test ranges from 1 to 10 depending on the last reached area, being the test performed in the order indicated in Table 1 . 
Table 1. Scores according to the Kapandji opposition test (KOT).

\begin{tabular}{ccl}
\hline Score & Finger & Area of contact \\
\hline 1 & Index & Lateral side of the second phalanx \\
2 & Index & Lateral side of the third phalanx \\
3 & Index & Tip \\
4 & Middle & Tip \\
5 & Ring & Tip \\
6 & Little & Tip \\
7 & Little & DIP crease \\
8 & Little & PIP crease \\
9 & Little & Proximal crease \\
10 & - & Distal volar crease of the hand \\
\hline
\end{tabular}

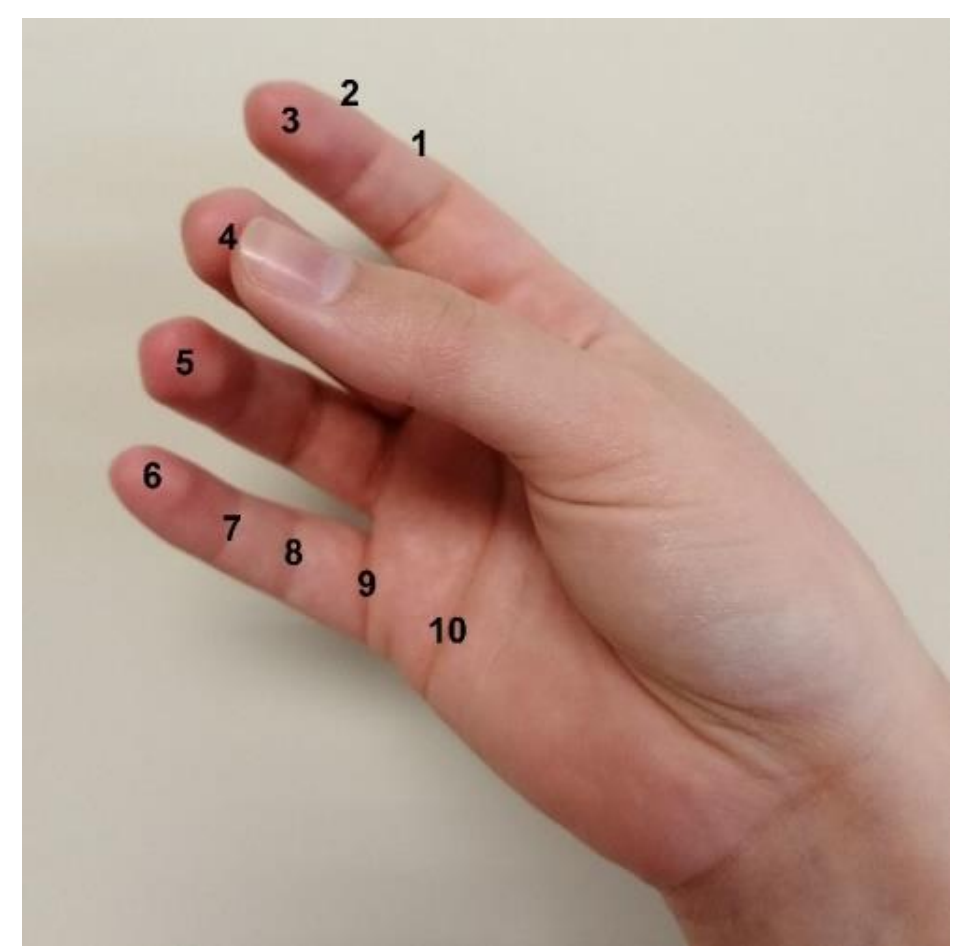

Figure 3. Areas to touch with the thumb tip in the Kapandji opposition test (KOT).

Grebenstein et al. (Grebenstein et al., 2010) considered that the KOT includes motion of the fingers and the thumb sufficient to evaluate the manipulation abilities. Other authors used the KOT to evaluate the functionality and anthropomorphism of artificial hands. Shin et al. (Shin et al., 2012) used the KOT to analytically analyze a new dexterous robot hand for delicate object grasping. Chalon et al. (Chalon et al., 2014) used the KOT to optimize the thumb of the Awiwi Hand obtaining the maximum score at KOT. Roa et al. (Roa et al., 2014) explored the relationship between kinematic design and manipulation performance of robotic hands, to analyze it they performed the KOT on seven thumb placements of a modular robotic 


\section{4 | Chapter 6}

hand. Deimel et al. (Deimel and Brock, 2016) also assessed the dexterity of the opposable thumb of a soft robotic hand using the KOT. Cerruti et al. (Cerruti et al., 2015) used the KOT to validate the thumb base placement of a kinematic model of an anthropomorphic robotic hand used for gesturing and grasping.

Some of the above mentioned studies that used the KOT made some adaptation of the test. For example, in some studies the authors did not consider the positions 1 and 2 corresponding to contact of the thumb with the lateral side of the index (Deimel and Brock, 2016; Roa et al., 2014) or removed some positions in the little finger (Deimel and Brock, 2016). Contrary, in some cases additional positions in the middle and ring fingers are included (Deimel and Brock, 2016; Roa et al., 2014). In most of these studies the KOT is only used to evaluate different hand designs. In some of them the authors used the KOT to optimize the thumb base placement (Cerruti et al., 2015; Chalon et al., 2014; Roa et al., 2014). In (Chalon et al., 2014) the optimization of the thumb included also as parameters the orientation of the joints, but the details about the cost function are not provided. To our knowledge, no previous study made an optimization of the TKC including simultaneously base placement, link lengths and all the joint orientation angles.

Following these approaches, in this study the objective was to define a computational method to optimize the whole TKC (base placement, link lengths and joint orientation angles) of an artificial hand based on its performance in the KOT. This method could be useful to improve the design of prosthetic and robotic hands regarding thumb opposition, leading to a better object grasping and manipulation. The application of the method involves defining an index, used in the cost function for optimization, which provides a metric for rating thumb opposition in artificial hands. To test the method we applied it to a 3D-printed prosthetic hand developed by the authors: the IMMA hand (Llop-Harillo and Pérez-González, 2017).

\section{Materials and Methods}

\subsection{IMMA Hand}

The IMMA hand (Llop-Harillo and Pérez-González, 2017) is a low-cost tendon-driven anthropomorphic prosthetic hand designed by the authors. It has five fingers with three phalanges per finger and 6 DoFs in total: 
independent flexion/extension in each of the four long fingers, and two independent DoFs for the thumb. The MCP and IP joints of the thumb are actuated both with the same tendon for flexion and the CMC joint is actuated by a separate tendon for circumduction. Figure 4 shows the TKC of the IMMA hand. Figure 5 shows the achievable target areas of the KOT by the right IMMA hand prototype. As is shown, its score is 4, because the positions 5 to 10 (see Table 1) cannot be reached.

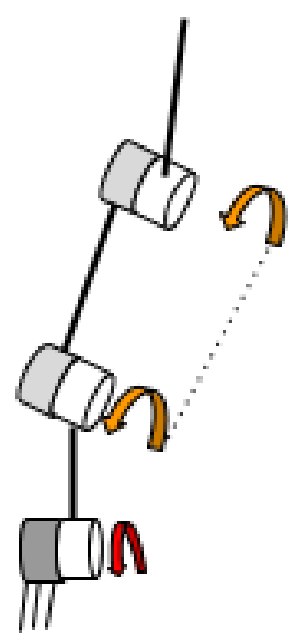

Figure 4. Thumb's kinematic chain (TKC) of the IMMA hand.
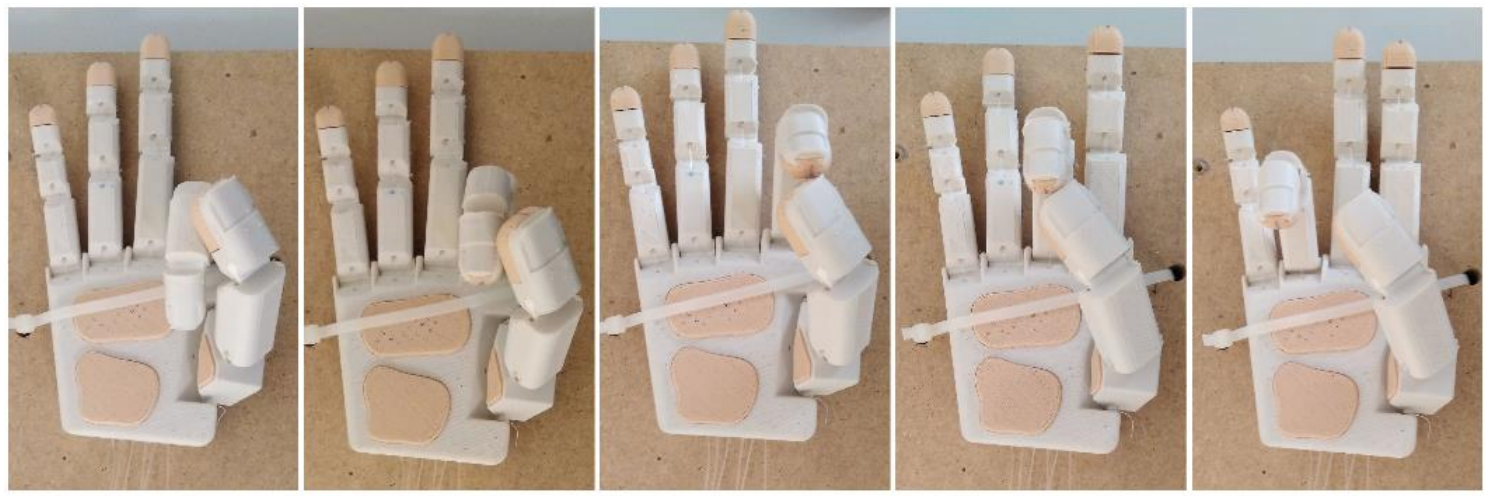

Figure 5. IMMA hand performing the Kapandji opposition test (KOT) in positions 1 (left) to 5 (right).

\subsection{Computational Model}

\subsubsection{Hand Model}

The model of the hand used in this study has a maximum of four straight links for each digit and a maximum of five digits. To define the kinematic chain of the hand, a local coordinate system (LCS) for each link has to be defined. In this study the LCS of each link is located in the middle of the 


\section{6 | Chapter 6}

joint with the proximal link. The LCSs were defined under the following criteria: Z-axis coincident with the flexion/extension axis of the joint, and oriented so that the flexion motion corresponds to a positive rotation around $\mathrm{Z}, \mathrm{X}$-axis aligned in palmar-dorsal direction pointing dorsally, thus indicating the abduction/adduction axis in the joint and Y-axis defining a right-handed coordinate system with the previous ones, resulting in a distal direction, in other words, pointing in the direction towards the tip of the fingers. This convention for the orientation of the axes is similar to that proposed by the ISB (Wu and Cavanagh, 1995), with the difference that the $\mathrm{X}$ and $\mathrm{Y}$ axes have opposite positive directions. With this selection the position of each LCS relative to the proximal one in the kinematic chain presents positive values in the translation along the $\mathrm{Y}$ axis.

Therefore, the kinematic chain of the hand is defined in the reference position with the three displacements and rotations of each LCS with respect to the immediate proximal in the chain. The wrist is taken as the fixed LCS for all the digits. Specifically, the LCS of link $j$ (1: metacarpus, 2: proximal phalanx, 3: intermediate phalanx, 4: distal phalanx) of the digit $i$ (1: thumb, 2: index, 3: middle, 4: ring, 5: little) is defined with the Equation 1.

$$
v_{i, j}=\left[\begin{array}{llllll}
x_{i, j} & y_{i, j} & z_{i, j} & \theta_{x_{i, j}} & \theta_{y_{i, j}} & \theta_{z_{i, j}}
\end{array}\right], \quad i=1,2 \ldots, 5, \quad j=1,2 \ldots, 5
$$

where the first three elements of the vector correspond to the translation vector of $L C S_{i, j}$ with respect to $L C S_{i, j-1}$ and the last three to the Euler angles with sequence of rotations XZY to orient $\operatorname{LCS}_{\mathrm{i}, \mathrm{j}-1}$ as $L C S_{\mathrm{i}, \mathrm{j}}$. At each finger, a last LCS ( $\mathrm{j}=5$ ) is added, positioned at its end (fingertip), with its $\mathrm{Y}$ axis in the proximal-distal direction and its $\mathrm{X}$ axis in the palmar-dorsal direction.

For each digit a maximum of six DoFs can be included in the hand model, two in CMC and MCP joints and one in the IP joints. Universal joints are considered in those with two DoFs. The hand position can be obtained straightforward by direct kinematics using the Equation 1 and the rotation angles in the joints.

\subsubsection{Cost Function}

To optimize the TKC we defined a cost function based on the KOT postures. We defined the position error $\varepsilon_{i}$ for each posture $i$ of the KOT as the minimum possible distance between the thumb tip and the corresponding target point of the test while the hand is moved within its workspace (Equation 2).

$$
\varepsilon_{i}=\min \left(\operatorname{dist}\left(p_{i}, p_{t}\right)\right)
$$


where $p_{i}$ is the target point, $p_{t}$ the thumb tip point, dist is a function defining the distance between two points and min is a function obtaining the minimum possible value of dist when moving the hand within its workspace.

Given a hand geometry and the range of motion of their joints, the calculation of the $\varepsilon_{i}$ involves an optimization. The variables for this optimization are the joint rotation angles of the hand. If these angles are coupled with a linkage, the number of variables for the optimization can be reduced, because the coupled rotation angles can be obtained from the coupling equations.

The final cost function for the optimization of the TKC was defined with Equation 3 as a relative mean position error (MPE) for the different positions of the KOT.

$$
M P E=\sum_{i} \varepsilon_{i} w_{i}
$$

where $w_{i}$ is a weighting coefficient for the posture $i$ of the KOT. Dividing the lengths of the kinematic chain by the hand length is convenient for having a non-dimensional index and making the evaluation independent of the hand size.

\subsubsection{Optimization algorithm}

For optimizing the TKC based on the KOT, the MPE above defined (Equation 3) has to be minimized, being the optimization variables the parameters defining the TKC: base placement, joint angles orientation and links length. Depending on the designer interest, it is also possible to limit the variables to only some of those defining the TKC. As the calculation of the minimum MPE requires the previous computation of the position errors $\varepsilon_{i}$ (Equation 2), the computational model involves two nested optimizations. Matlab was used in this study for the computation. The built-in Genetic Algorithm ('ga') was used for the optimization of the TKC whereas 'fmincon' function for non-linear optimization was used for the nested optimization corresponding to each position of the KOT. Table 2 shows the pseudocode used in the computation model. 
Table 2. Pseudocode for optimizing the TKC.

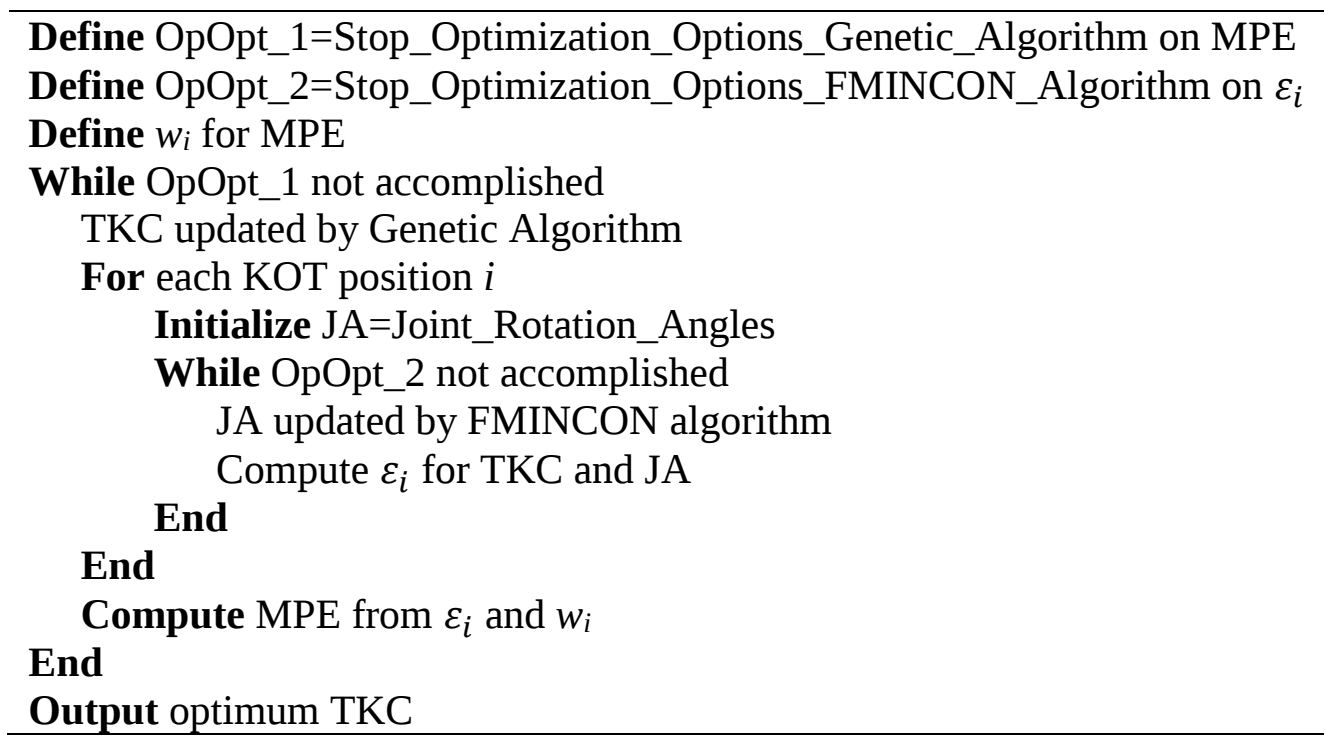

\subsection{Case study: Optimization of the TKC of the IMMA hand}

A simplified model of the IMMA hand (Llop-Harillo and PérezGonzález, 2017) was created in Matlab (Figure 6). Table 3 shows the components of the translation-rotation vectors that define the kinematic chain of the hand (vectors $v_{i, j}$, Equation 1 ), where $x, y, z$ are non-dimensional values related to the hand length (distance from the wrist to the end of the middle finger) and $\theta_{x}, \theta_{y}, \theta_{z}$ angles are the Euler rotations around the $\mathrm{X}, \mathrm{Y}$, $\mathrm{Z}$ axes, respectively, with rotation order $\mathrm{XZY}$, expressed in radians. The joints range of motion (ROM) were defined based on the hand prototype (Figure 5) and are shown in Table 4. For the abduction/adduction movement in the MCP joints we included a small ROM accounting for the flexibility of the joints in the prototype. 
Table 3. Data for the kinematic chain of the IMMA hand according to Equation 1 (lengths are non-dimensional values related to the hand length and Euler angles are in radians).

\begin{tabular}{|c|c|c|c|c|c|c|}
\hline Links & $v_{i, j}$ & Thumb & Index & Middle & Ring & Little \\
\hline \multirow[t]{6}{*}{ Metacarpal } & $x$ & 0 & 0 & 0 & 0 & 0 \\
\hline & $y$ & 0.2169 & 0 & 0 & 0 & 0 \\
\hline & $z$ & 0.1577 & 0 & 0 & 0 & 0 \\
\hline & $\theta x$ & 1.5708 & 0 & 0 & 0 & 0 \\
\hline & $\theta y$ & 0 & 0 & 0 & 0 & 0 \\
\hline & $\theta z$ & 0 & 0 & 0 & 0 & 0 \\
\hline \multirow[t]{6}{*}{ Proximal phalanx } & $x$ & 0 & 0 & 0 & 0 & 0 \\
\hline & $y$ & 0.1320 & 0.4588 & 0.4370 & 0.4192 & 0.3874 \\
\hline & $z$ & 0 & 0.1188 & 0.0016 & -0.1054 & -0.2004 \\
\hline & $\theta x$ & 0 & 0.0873 & 0 & -0.1250 & -0.2618 \\
\hline & $\theta y$ & -0.7854 & 0 & 0 & 0 & 0 \\
\hline & $\theta z$ & 0 & 0 & 0 & 0 & 0 \\
\hline \multirow[t]{6}{*}{ Intermediate phalanx } & $x$ & 0 & 0 & 0 & 0 & 0 \\
\hline & $y$ & 0.2158 & 0.2456 & 0.2725 & 0.2291 & 0.1968 \\
\hline & $z$ & 0 & 0 & 0 & 0 & 0 \\
\hline & $\theta x$ & 0 & 0 & 0 & 0 & 0 \\
\hline & $\theta y$ & 0 & 0 & 0 & 0 & 0 \\
\hline & $\theta z$ & 0 & 0 & 0 & 0 & 0 \\
\hline \multirow[t]{6}{*}{ Distal phalanx } & $x$ & 0 & 0 & 0 & 0 & 0 \\
\hline & $y$ & 0.1659 & 0.1399 & 0.1623 & 0.1509 & 0.0590 \\
\hline & $z$ & 0 & 0 & 0 & 0 & 0 \\
\hline & $\theta x$ & 0 & 0 & 0 & 0 & 0 \\
\hline & $\theta y$ & 0 & 0 & 0 & 0 & 0 \\
\hline & $\theta z$ & 0 & 0 & 0 & 0 & 0 \\
\hline \multirow[t]{6}{*}{ Fingertip } & $x$ & 0 & 0 & 0 & 0 & 0 \\
\hline & $y$ & 0 & 0.1243 & 0.1324 & 0.1324 & 0.1135 \\
\hline & $z$ & 0 & 0 & 0 & 0 & 0 \\
\hline & $\theta x$ & 0 & 0 & 0 & 0 & 0 \\
\hline & $\theta y$ & 0 & 0 & 0 & 0 & 0 \\
\hline & $\theta z$ & 0 & 0 & 0 & 0 & 0 \\
\hline
\end{tabular}

Table 4. Joints range of motion (minimum angle/maximum angle) in degrees for the IMMA hand (add/abd: adduction/abduction, ext/flex: extension/flexion).

\begin{tabular}{cccccc}
\hline Joint & Thumb & Index & Middle & Ring & Little \\
\hline CMC add/abd & $0 / 0$ & $0 / 0$ & $0 / 0$ & $0 / 0$ & $0 / 0$ \\
CMC ext/flex & $-10 / 70$ & $0 / 0$ & $0 / 0$ & $0 / 0$ & $0 / 0$ \\
MCP add/abd & $-1 / 1$ & $-1 / 1$ & $-1 / 1$ & $-1 / 1$ & $-1 / 1$ \\
MCP ext/flex & $-11 / 55$ & $-20 / 85$ & $-17 / 86.3$ & $-15 / 70$ & $-20 / 65$ \\
PIP ext/flex & $-13 / 55$ & $-20 / 60$ & $-17 / 75$ & $-20 / 75$ & $-20 / 65$ \\
DIP ext/flex & $0 / 0$ & $-15 / 50$ & $-20 / 75$ & $-20 / 70$ & $-20 / 75$ \\
\hline
\end{tabular}




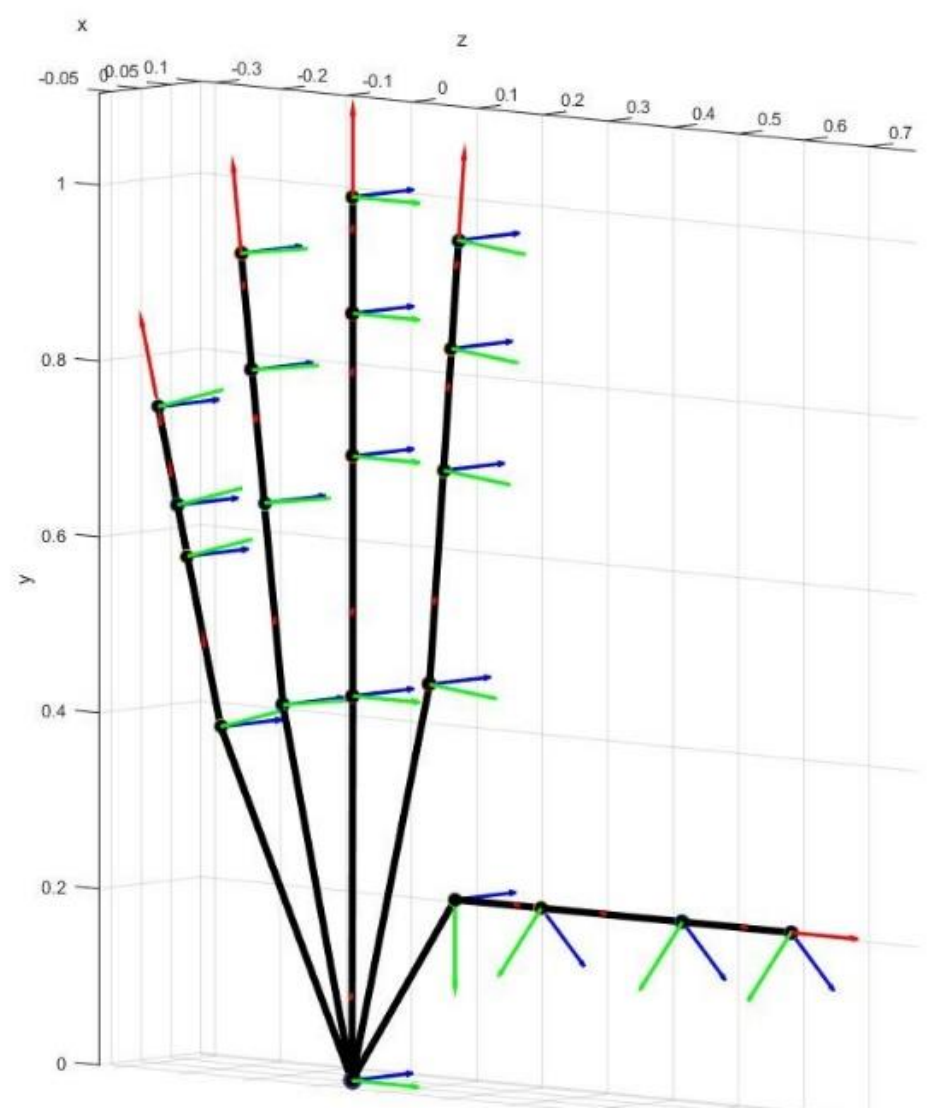

Figure 6. Simplified model of the right IMMA hand. Colored arrows in the joints indicate local coordinate systems (LCSs): green for Z-axis, red for Y-axis, blue for X-axis.

For the optimization of the TKC in this case study the position 10 of the KOT (see Figure 3) was not considered because it was difficult to locate in a simplified model of the hand. The positions 1 and 2 where considered in the more proximal point of the corresponding index phalanx. In Equation 3 positions considered were weighted equally, so we used $w_{i}=1 / 9$ for $i=1$ to 9 and $w_{10}=0$. Moreover, in this case the joint rotation angles of the hand were considered independent, without taking into account the coupling equations resulting from the actuation of several joints with a same tendon. The variables for the optimization were the orientation of the CMC and MCP joints, the thumb's links length and the position of the CMC joint. The feasible range of these variables, upper and lower bounds for the optimization, is shown in Table 5. The default optimization options were considered for the genetic algorithm of Matlab (' $g a$ ') except 'FitnessLimit' and 'FunctionTolerance' both set to 0.001 and 'PopulationSize' set to 50. For the non-linear optimization with Matlab built-in function ('fmincon') the default optimization options were also considered except 'MaxFunctionEvaluations' set to 10000 and 'StepTolerance' set to 0.0001 . 
Table 5. Lower and upper bounds (Min/Max) of the optimization variables in this case study (lengths are non-dimensional values related to the hand length and Euler angles are in radians).

\begin{tabular}{lccl}
\hline$v_{i, j}$ component & Min & Max & Anatomical meaning \\
\hline$x_{1,1}$ & -0.3 & 0 & CMC position \\
$y_{1,1}$ & 0.1 & 0.3 & \\
$z_{1,1}$ & 0.1 & 0.3 & \\
$\theta x_{1,1}$ & $\pi / 2$ & $\pi$ & CMC orientation \\
$y_{1,2}$ & 0.1 & 0.3 & Metacarpal length \\
$\theta y_{1,2}$ & $-\pi / 2$ & $\pi / 2$ & MCP orientation \\
$y_{1,3}$ & 0.1 & 0.3 & Proximal phalanx length \\
$y_{1,4}$ & 0.1 & 0.3 & Distal phalanx length \\
\hline
\end{tabular}

\section{Results}

Figure 7 shows the position error $\left(\varepsilon_{i}\right.$, Equation 2$)$ for the the initial IMMA hand and for the IMMA hand after optimizing the TKC following the method indicated in Table 2. According to the simplified model the position 1 of the KOT is not reachable by the original hand nor by the improved one. Without considering this position, the score in the KOT improved from 3 in the original hand to 5 in the model with optimized TKC. The position error for positions 6 to 8 improved significantly. The MPE in the optimized model was reduced to about one third with respect to the original model $(0.121$ to $0.035)$.

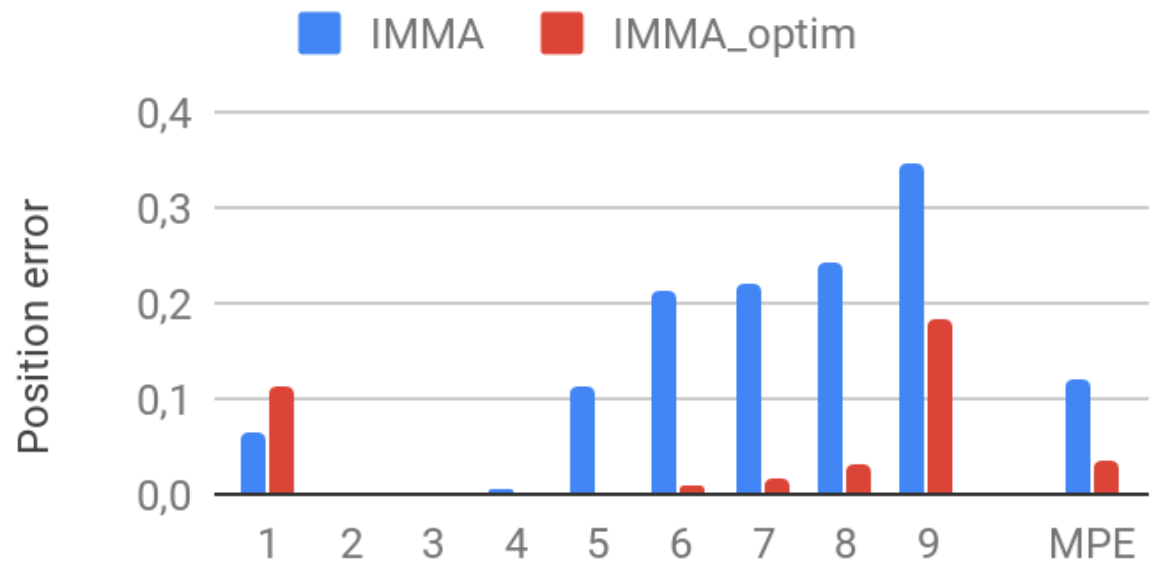

\section{Posture KOT}

Figure 7. Position error ( $\varepsilon_{i}$, Equation 2$)$ for each posture of KOT and mean position error (MPE).

The kinematic chain of the improved hand can be seen in Figure 8 and the Table 6 shows the comparison of the initial and optimized values of the parameters for the TKC. From the comparison of the thumb in Figure 6 and 
Figure 8 it can be observed that the base placement of the thumb, i.e. the CMC joint, is closer to the center of the palm in the optimized design, the orientation of the MCP joint is slightly varied and the phalanges length has changed, being the distal phalanx longer in the optimized design.

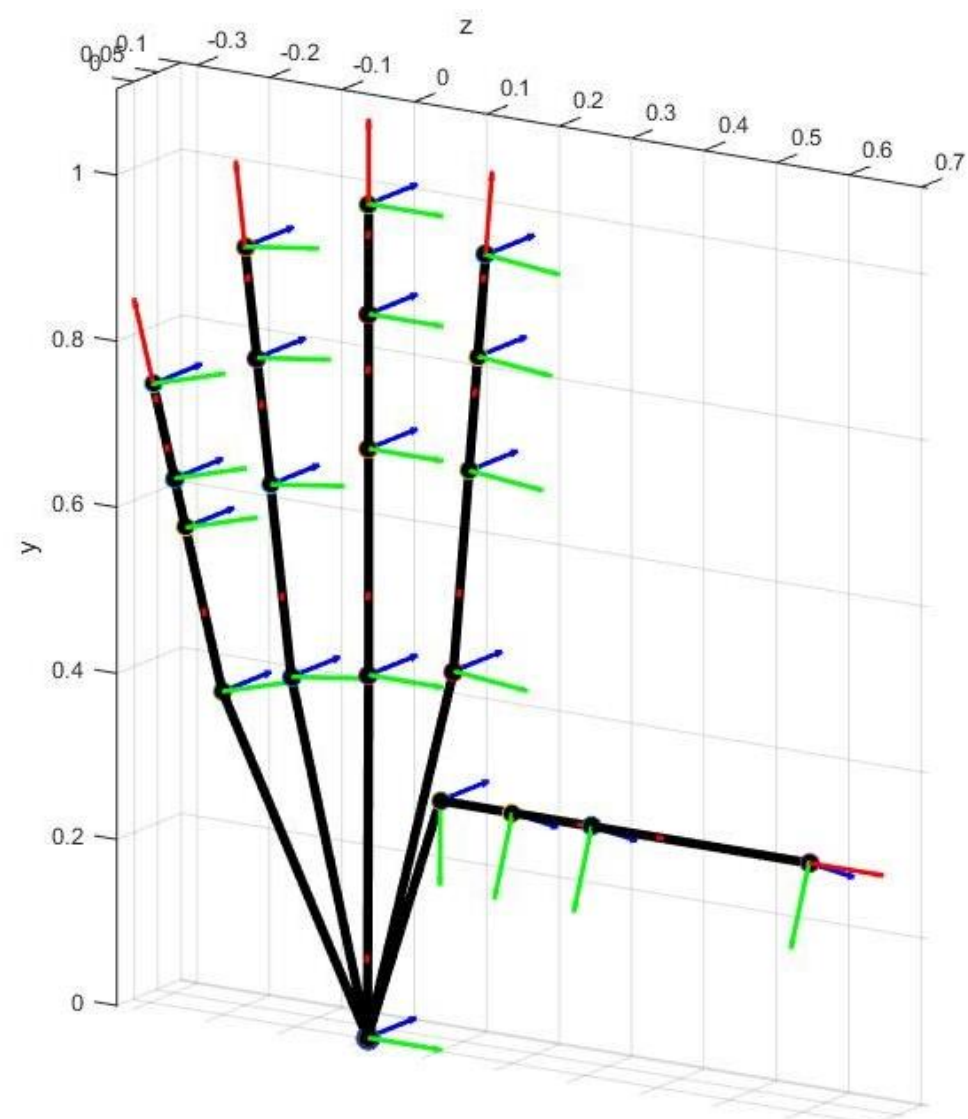

Figure 8. Kinematic chain of the right IMMA hand with optimized TKC.

Table 6. Initial and optimized parameters of the TKC for the IMMA hand (lengths are non-dimensional values related to the hand length and Euler angles are in radians).

\begin{tabular}{ccc}
\hline$v_{i, j}$ component & Initial & Optimized \\
\hline$x_{1,1}$ & 0.000 & 0.000 \\
$y_{1,1}$ & 0.2169 & 0.3000 \\
$z_{1,1}$ & 0.1577 & 0.1002 \\
$\theta x_{1,1}$ & 1.5708 & 1.5714 \\
$y_{1,2}$ & 0.1320 & 0.1000 \\
$\theta y_{1,2}$ & -0.7854 & -0.3855 \\
$y_{1,3}$ & 0.2158 & 0.1094 \\
$y_{1,4}$ & 0.1659 & 0.3000 \\
\hline
\end{tabular}

Fifty-nine generations were necessary in the genetic algorithm for the optimization of the TKC and the execution took about 10 hours in an Intel Core i7 2.6 GHz processor. Figure 9 shows the evolution of the mean and 
best fitness values, corresponding to MPE, for the different generations of the genetic algorithm. The 50 individuals of the last generation of the genetic algorithm were all very similar among them, representing quite similar TKCs.

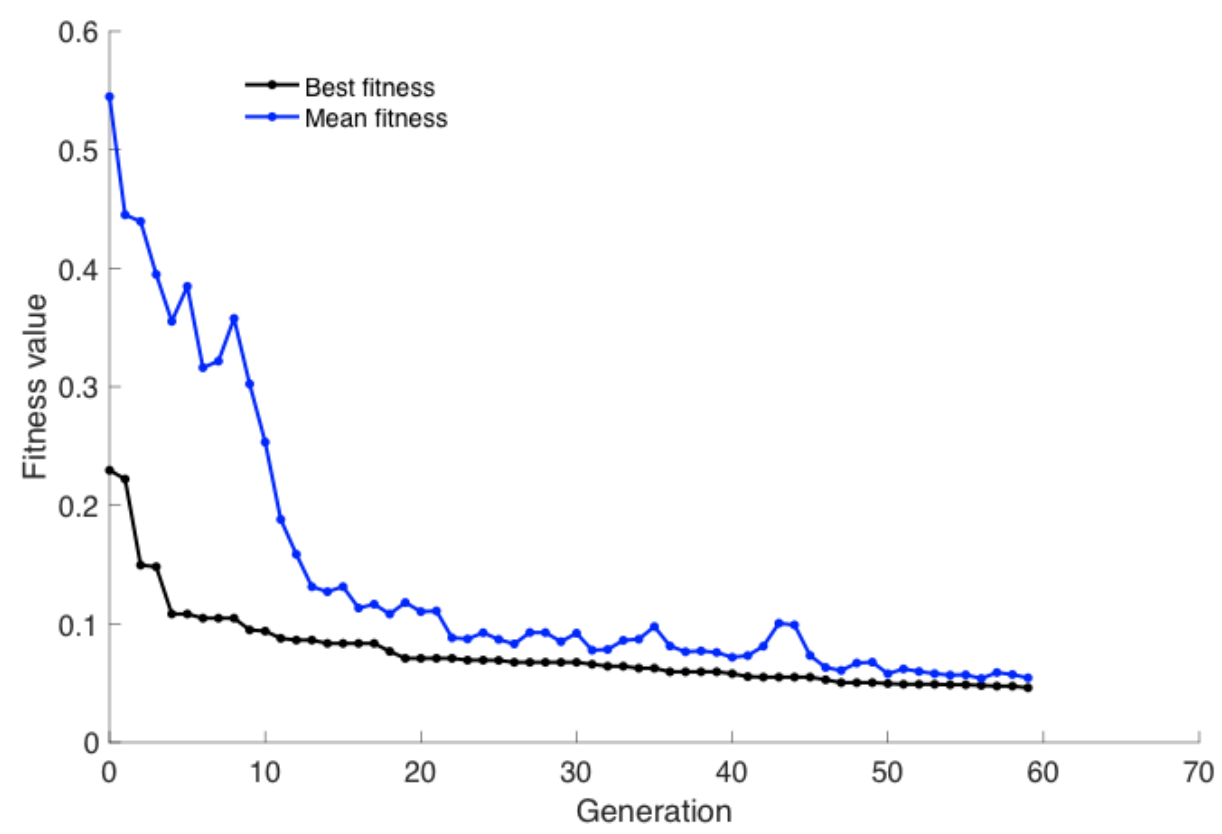

Figure 9. Fitness value (MPE) evolution among generations of the genetic algorithm for optimizing the thumb's kinematic chain (TKC).

\section{Discussion}

We have presented a new method to optimize the TKC of an artificial hand based on their performance in the KOT. Despite the KOT has been taken into account in previous studies for the design of artificial hands (Cerruti et al., 2015; Chalon et al., 2014; Roa et al., 2014) none of these previous studies presented a clear computational method able to do it following a formal optimization procedure and including all the parameters defining the TKC. The methodology presented here, using a double nested optimization method (Table 2) allows considering all the KOT postures for the optimization or only some of them. We have defined an index quantifying the mean weighted position error (MPE) based on the position error for each KOT posture, which can be useful as a method to compare the opposition performance of an artificial hand. The weight associated to each posture in this index can easily be tuned by the designer depending on their design specifications. Moreover, the optimization procedure allows including as variables any of the parameters defining the TKC or even other parameters of the kinematic chain of the hand. 


\section{4 | Chapter 6}

In this study the methodology was applied, as a case study, to the optimization of the TKC of the IMMA hand, a 3D-printed cable driven hand developed by the authors. The MPE in the optimized design was reduced to less than one third of that of the original design, showing the effectiveness of the computational method. Due to the simplification of the hand model, whose segments are considered as straight lines, the computational model is only an approximation to the real prototype and some differences can be observed in the KOT score obtained with the real prototype and with the model. As Figure 5 shows, the original prototype of the IMMA hand, can achieve the positions 1 to 4 of the KOT. Nevertheless, Figure 7 shows a nonnull position error in the model for positions 1 and 4 . This could be attributed to the fact that the width and thickness of the phalanges were neglected in the simplified model and also to the fact that positions 1 and 2 where considered in the more proximal point of the corresponding index phalanx. Taking this into account, the positions 2 to 8 in the improved model can be considered as reachable in practical terms, whereas only the positions 2 to 4 are clearly reachable in the initial hand. Regarding the position 1, it could also be reachable depending on the position taken for the target point in the middle phalange of the index finger (Figure 10).
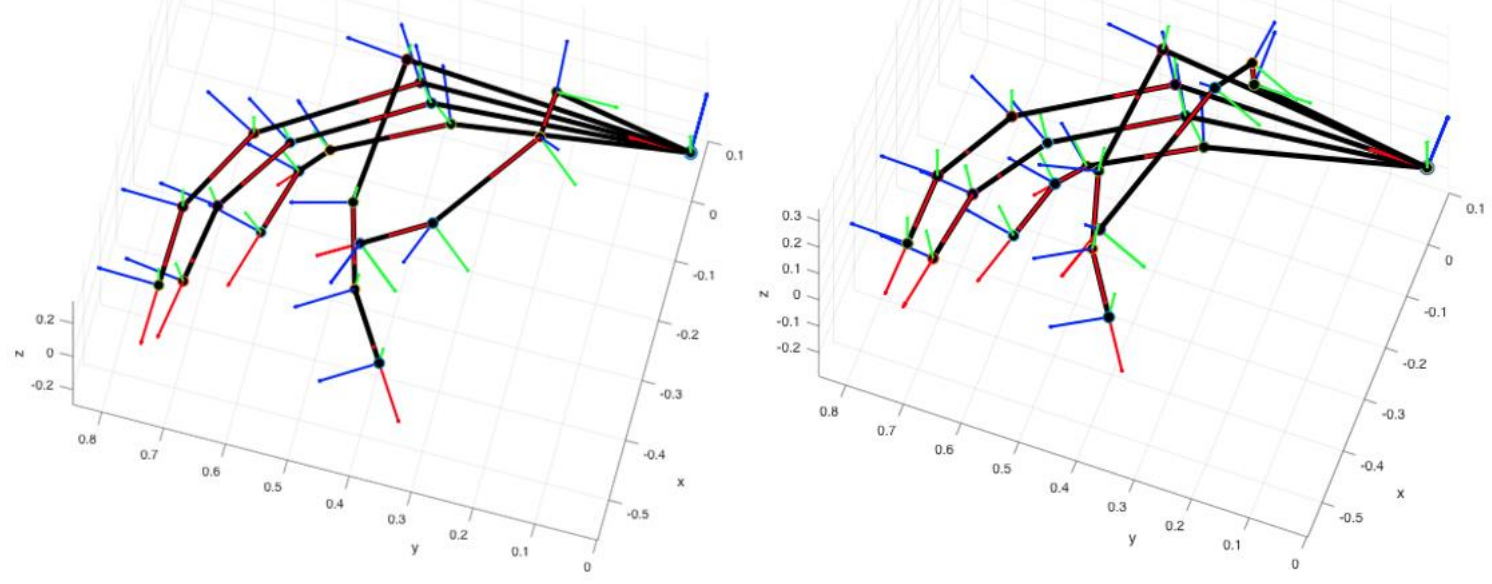

Figure 10. Posture 1 of the Kapandji opposition test (KOT). Left: original IMMA hand. Right: optimized IMMA hand.

Some of the parameters of the optimized model (Table 6) are in the upper or lower bounds selected in the optimization (Table 5), which could indicate that widening the allowable range for the parameters could produce TKC designs able to reduce even more the MPE. This has not been analyzed in the present study but is a possible future work. 
The optimized design obtained in this study has a thumb base location more distal, favoring the score in the KOT, but probably making more difficult grasping big objects. The total length of the optimized TKC is similar to that of the original design, but the proportion of the segments changed, with a longer distal phalanx and shorter metacarpal and proximal phalanges as compared to the original model. One possible reason for this is that this configuration helps to reduce the position error in the last postures of the KOT (7 to 9). We analyzed the changes in the results when the KOT positions included in the MPE are restricted to positions 1 to 6 , reducing the effect of little finger opposition in the MPE. Figure 11 shows a graphical comparison of the TKC parameters of the original design and the optimized designs including positions 1 to 9 or 1 to 6 in the MPE, as well as the upper and lower bounds considered for the optimization. The results indicate that the optimized TKCs including positions 1 to 9 or 1 to 6 are very similar.

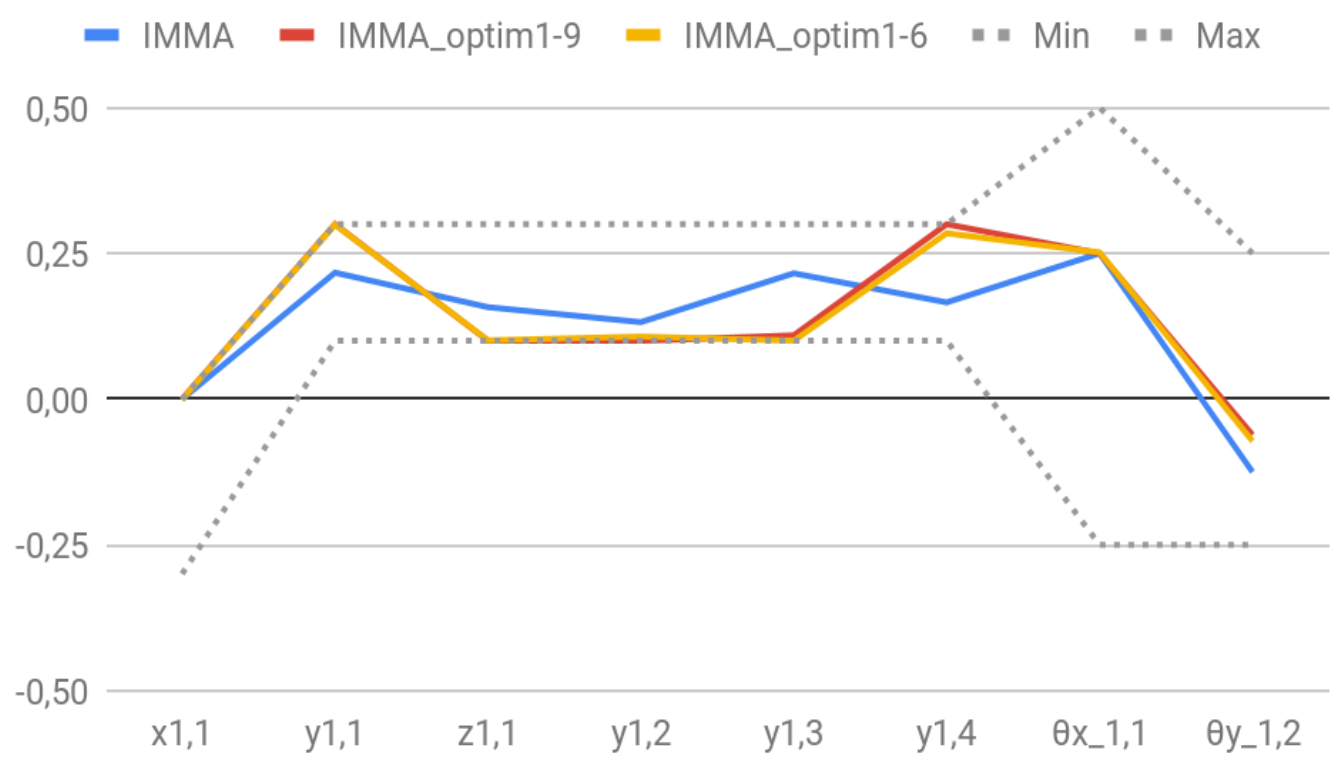

Figure 11. Thumb's kinematic chain (TKC) parameters (angles $\theta_{x_{-} 1,1}$ and $\theta_{y_{-} 1,2}$ divided by $2 \pi$ ) for the original design of the IMMA hand and for the optimized versions obtained including positions 1-9 or 1-6 in the MPE. Upper (Max) and

lower (Min) bounds for the optimization are shown with dotted lines.

Additional simulations in Matlab for the original IMMA hand and the optimized design suggest that the reduction of MPE in KOT does not guarantee a better design according to other criteria when comparing with the human hand. We compared both designs with three different anthropomorphic indexes of the kinematic chain of the whole hand (LlopHarillo et al., 2018) and the performance was similar, with differences lower to $3 \%$, for two of them: one based on the comparison of the kinematic chain 


\section{6 | Chapter 6}

with that of the human hand; a second based on grasping postures for some primitive objects (sphere, cylinder and prism). However, the optimized design had a worse performance, 25\% lower, in the index defined considering the intersection of the workspaces of the artificial hand and the human hand. This result is aligned with the observation of Roa et al. (Roa et al., 2014) about the difficulties to find direct correlations between the Kapandji test score and the size of the functional workspace. This aspect should be better investigated in the next future. The performance of the optimized design of the IMMA hand with respect to the original design should also be compared in the next future with physical prototypes, using grasping benchmarks.

The index based on the MPE defined in this study is complementary to other anthropomorphism indexes developed by the authors and existing in the literature. Probably an adequate combination of these indexes can help to improve the hand's kinematic chain and should be investigated, as well as the extension of the optimization to other parameters of the kinematic chain of the hand, not restricted to the thumb.

\section{Conclusion}

A straightforward methodology to analytically optimize the kinematic chain of the thumb of an artificial hand based on the performance in the KOT has been presented. The cost function defined for the optimization (MPE) is a weighted mean position error when trying to reproduce the KOT postures and can be used also as a metric to quantify thumb opposition in the hand. The application of the method to the IMMA hand thumb allowed defining a new TKC reducing the MPE to near one third of that of the original design and increasing significantly the number of reachable positions in the KOT. However, additional simulations showed that the optimized design could have a worse outcome according to other index considering the intersection between the workspace of the artificial hand and that of the human hand. Benchmarking grasping test on physical prototypes could give additional insights about the usefulness of the proposed methodology. The metric proposed based on the KOT can be used directly or in combination with other to improve the kinematic chain of artificial hands. 


\section{Acknowledgments}

This work was supported by the Spanish Ministry of Economy and Competitiveness and ESF [grant number BES-2015-076005]; Spanish Ministry of Economy and Competitiveness, AEI and ERDF [grant numbers DPI2014-60635-R, DPI2017-89910-R]; and Universitat Jaume I [grant number UJI-B2017-70].

\section{References}

Bardo, A., Vigouroux, L., Kivell, T.L., Pouydebat, E., 2018. The impact of hand proportions on tool grip abilities in humans, great apes and fossil hominins: A biomechanical analysis using musculoskeletal simulation. Journal of Human Evolution, 125, 106-121.

Belter, J.T., Segil, J.L., Dollar, A.M., Weir, R.F., 2013. Mechanical design and performance specifications of anthropomorphic prosthetic hands: A review. The Journal of Rehabilitation Research and Development, 50(5), 599-618.

Cerruti, G., Chablat, D., Gouaillier, D., Sakka, S., 2015. Design method for an anthropomorphic hand able to gesture and grasp, in: 2015 IEEE International Conference on Robotics and Automation (ICRA). IEEE, pp. 3660-3667.

Chalon, M., Dietrich, A., Grebenstein, M., 2014. The Thumb of the Anthropomorphic Awiwi Hand: From Concept to Evaluation. International Journal of Humanoid Robotics, 11(3), 1450019.

Coert, J.H., van Dijke, G.A.H., Hovius, S.E.R., Snijders, C.J., Meek, M.F., 2003. Quantifying thumb rotation during circumduction utilizing a video technique. Journal of Orthopaedic Research, 21(6), 1151-1155.

Deimel, R., Brock, O., 2016. A novel type of compliant and underactuated robotic hand for dexterous grasping. The International Journal of Robotics Research, 35(1-3), 161-185.

Feix, T., Kivell, T.L., Pouydebat, E., Dollar, A.M., 2015. Estimating thumbindex finger precision grip and manipulation potential in extant and fossil primates. Journal of The Royal Society Interface, 12(106), 2015017620150176.

Gaiser, I., Schulz, S., Kargov, A., Klosek, H., Bierbaum, A., Pylatiuk, C., Oberle, R., Werner, T., Asfour, T., Bretthauer, G., Dillmann, R., 2008. A new anthropomorphic robotic hand, in: Humanoids 2008 - 8th IEEE-RAS International Conference on Humanoid Robots. IEEE, pp. 418-422.

Giurintano, D.J., Hollister, A.M., Buford, W.L., Thompson, D.E., Myers, L.M., 1995. A virtual five-link model of the thumb. Medical engineering \& physics, 17(4), 297-303. 


\section{8 | Chapter 6}

Grebenstein, M., Chalon, M., Hirzinger, G., Siegwart, R., 2010. A Method for Hand Kinematics Designers 7 Billion Perfect Hands. 1st International Conference on Applied Bionics and Biomechanics.

Kapandji, A., 1986. Clinical opposition and reposition test of the thumb [COTATION CLINIQUE DE L'OPPOSITION ET DE LA CONTREOPPOSITION DU POUCE]. Annales de Chirurgie de la Main, 5(1), 67-73.

Liu, H., Wu, K., Meusel, P., Seitz, N., Hirzinger, G., Jin, M.H., Liu, Y.W., Fan, S.W., Lan, T., Chen, Z.P., 2008. Multisensory five-finger dexterous hand: The DLR/HIT Hand II, in: 2008 IEEE/RSJ International Conference on Intelligent Robots and Systems. IEEE, pp. 3692-3697.

Llop-Harillo, I., Pérez-González, A., 2017. System for the experimental evaluation of anthropomorphic hands. Application to a new 3D-printed prosthetic hand prototype. International Biomechanics, 4(2), 50-59.

Llop-Harillo, I., Pérez-González, A., Andrés de la Esperanza, F.J., 2018. Comparación de la cadena cinemática de manos antropomorfas artificiales con la de la mano humana, in: Anales de Ingeniería Mecánica. Revista de La Asociación Española de Ingeniería Mecánica. Actas Del XXII CONGRESO NACIONAL DE INGENIERÍA MECÁNICA. Madrid, pp. 49-68.

Ottobock, 2017. Ottobock myoelectric prosthetics. https://www.ottobockus.com/prosthetics/upper-limb-prosthetics/solutionoverview/myoelectric-prosthetics/ [3.01.19].

Roa, M.A., Chen, Z., Staal, I.C., Muirhead, J.N., Maier, A., Pleintinger, B., Borst, C., Lii, N.Y., 2014. Towards a functional evaluation of manipulation performance in dexterous robotic hand design, in: 2014 IEEE International Conference on Robotics and Automation (ICRA). IEEE, pp. 6800-6807.

Santos, V.J., Valero-Cuevas, F.J., 2006. to Multimodal Distributions of DenavitHartenberg Parameters for the Human Thumb. IEEE Transactions on Biomedical Engineering, 53(2), 155-163.

Shadow Robot Company, 2019. Shadow Dexterous Hand. https://www.shadowrobot.com/products/dexterous-hand/ [3.01.19].

Shin, S., Choi, D., Choi, M., Moon, H., Choi, H.R., Koo, J.C., 2012. Development of dexterous robot hand for delicate object grasping, in: 2012 9th International Conference on Ubiquitous Robots and Ambient Intelligence (URAI). IEEE, pp. 462-463.

ten Kate, J., Smit, G., Breedveld, P., 2017. 3D-printed upper limb prostheses: a review. Disability and Rehabilitation: Assistive Technology, 12(3), 300-314.

Wu, G., Cavanagh, P.R., 1995. ISB recommendations for standardization in the reporting of kinematic data. Journal of Biomechanics, 28(10), 1257-1261. 


\section{Chapter 7}

\section{Benchmarking prosthetic hands through anthropomorphic grasping simulations}



This chapter corresponds exactly to the manuscript submitted to Bioinspiration \& Biomimetics: Benchmarking prosthetic hands through anthropomorphic grasping simulations Immaculada Llop-Harillo, José L. Iserte, and Antonio Pérez-González *Currently working on a resubmission

\begin{abstract}
In recent decades, the design of anthropomorphic hands has been developed greatly improving both cosmesis and functionality. Experimentation, simulation and combined approaches have been used in the literature to assess the effect of design alternatives (DAs) on the final performance of artificial hands. However, establishing standard benchmarks for grasping and manipulation is a need recognized among the robotics community. Experimental approaches are costly, time-consuming and inconvenient in early design stages. Alternatively, computer simulation with the adaptation of metrics based on experimental benchmarks for anthropomorphic hands could be useful to evaluate and rank DAs. The aim of this study is to compare the anthropomorphism of the grasps performed with thirty DAs of the IMMA hand, developed by the authors, using either: 1) the brute-force approach and grasp quality metrics proposed in previous works; 2) a preliminary approach to a new simulation benchmark. The new methodology involves the use of human knowledge for the generation of efficient grasp hypotheses and the definition of a new metric to assess stability and human likeness adapting to the simulation environment the experimental Anthropomorphic Hand Assessment Protocol. This new benchmark, in contrast to the other approach, resulted in anthropomorphic and more realistic grasps for the expected use of the objects. Despite the inherent limitations of a simulation analysis, the benchmark proposed provides interesting results for selecting optimal DAs in order to perform stable and anthropomorphic grasps for the included grasp types: pulp pinch and cylindrical grip.
\end{abstract}

\title{
Keywords
}

Analytical assessment, anthropomorphism, benchmarking, grasping, prosthetic hand, simulation 


\section{2 | Chapter 7}

\section{Introduction}

Artificial hands are used in both robotics and prosthetics to provide grasping abilities to either robots or people with upper-limb limitations. Anthropomorphic hands are becoming popular, especially in the last decades, driven by the need to improve cosmesis but also as a means to improve the functionality. However the unmatched functionality and aesthetics of the human hand is still far to be achieved with current technology (Belter et al., 2013; Jang et al., 2011). Among the different factors that limit the functionality of current artificial hands for grasping or manipulating objects, one of the first to be considered in design is the kinematic chain configuration. Specifically, the number of segments per finger and the orientation of the thumb and finger joints affect the achievable grasping postures and thus the final functionality.

Experimentation, simulation and combined approaches have been used in the past to analyze the effect of the artificial hand design on its final performance. Experimental approaches allow assessing the grasping ability of artificial hands with realistic information about the final performance. Establishing standard benchmarks for grasping and manipulation is a need recognized among the robotics community (Calli et al., 2015; Falco et al., 2015). Quispe et al. (Huamán Quispe et al., 2018) proposed a general taxonomy for benchmarking manipulation tasks and described recommendations about how to define useful testing protocols. The authors have recently proposed a protocol (Llop-Harillo et al., 2019) to quantify functionality and human-like grasping of anthropomorphic hands while grasping objects of daily living. However, experimental approaches are costly and time-consuming and require the use of physical prototypes, which is inconvenient in early design stages. Alternatively, computer simulation can be useful to evaluate and rank design alternatives (DAs). Some studies (Feix et al., 2013; Liarokapis et al., 2013) proposed anthropomorphism indexes based on the comparison of the workspace of the artificial hand and that of the human hand. However, a limitation of this approach is the fact that the indexes are based only on the comparison of the reachable positions for the hand but do not consider the position of all the contact points of the hand with actual objects. Consequently, the final grasp stability obtained with different hand designs cannot be evaluated.

In robotics, the problems of grasp simulation and grasp planning have been extensively analyzed (Sahbani et al., 2012). Grasp planning involves determining a hand configuration and a set of feasible contact points between 
the hand and the object to be grasped in order to reach force-closure, equilibrium or stability, among other possible objectives. In order to decide the best grasping strategy, quantification of the grasp quality of a given object-hand-posture set is needed and different grasp quality metrics (GQM) have been proposed in the literature. Roa and Suarez (Roa and Suárez, 2014) made an extensive survey of most of these metrics. Rubert and Morales (Rubert and Morales, 2016) compared the use of ten selected GQM with the use of the anthropomorphism index proposed by Feix et al. (Feix et al., 2013) in order to evaluate the performance of different anthropomorphic hands, concluding that both approaches are not equivalent. In that work, they noted also a low correlation among the ten GQM when evaluating the same set of grasps. A low correlation among GQM was also found in a different study of the same authors with other hands and objects (Rubert et al., 2017). The use of combined metrics has been suggested as a method to find a more robust estimator of the grasp quality (Roa and Suárez, 2014), although an optimal solution has not been found. Despite these limitations, the use of GQM for evaluating the ability of a robotic or prosthetic hand design for achieving successful grasps can be considered as an alternative to costly experimentation and prototyping in the initial design stages.

A rather limited number of simulation tools have been proposed in the past in the robotics community for grasping simulation. GraspIt (Miller and Allen, 2004) is one of the pioneering tools made available to researchers (http://graspit-simulator.github.io/), and included the models for some popular robot hands. OpenRAVE (Diankov, 2010) is a general environment for testing, developing, and deploying motion planning algorithms in realworld robotics applications. With respect to GraspIt, OpenRAVE has the advantage of having a modular design, simplifying extension and further development. The open architecture and modular design allows a simple integration of simulation, visualization, planning, scripting and control of robot systems. OpenGrasp (http://opengrasp.sourceforge.net/) (León et al., 2010) was developed as a toolkit to simulate grasping within the framework of OpenRAVE (http://openrave.org/). OpenGrasp completes OpenRAVE including improvements in the use of different physics simulation engines and also incorporates a robot editor based on the use of the COLLADA ${ }^{\mathrm{TM}}$ file format (“COLLADA,” n.d.). A version of OpenGrasp (OpenHand) including also a biomechanical model to simulate grasping with the human hand was later developed by León (León et al., 2014) in collaboration with the authors' research group. This version is publicly available in https://sites.google.com/a/uji.es/devalhand/openhand-simulator. OpenHand 


\section{4 | Chapter 7}

presents a graphical user interface for automatic generation of grasp hypotheses (GHs) and implements the computation of several GQM to evaluate the final grasps.

In OpenRAVE the grasping problem can be faced with the brute-force paradigm by the use of its Grasping Module (Rubert et al., 2017). For generating the GHs between object and hand, a cartesian grid of points is generated in the surface of a bounding box around the object and projected over its surface. From these points on the surface, a series of approach rays are generated, defining different grasps approaches. This generation of grasps is dependent on several parameters such as: the distance between the points in the cartesian grid of the bounding box; the angle between the approach rays and the normal to the object surface; the standoff distance at which the palm of the hand stands still and the grip begins; and the angle to rotate the hand about the approach ray. For a given set of values for these parameters, a set of GHs, initial positions and orientations of the hand with respect to the object, is generated.

The brute-force approach has the advantage of being general and applicable to any hand or object, being useful for facing the grasp planning in a robotic environment (Levine et al., 2018; Morales et al., 2004; Pinto and Gupta, 2016). However, the use of this method presents some major drawbacks. Firstly, it requires a long time to compute a reasonable sample. Secondly, some of the GHs generated are redundant for objects presenting some kind of symmetry. Thirdly, and particularly relevant in applications such as prosthetics, service robotics and human-robot cooperation, most of the final grasps obtained are not realistic for activities of daily living (ADLs). Moreover, for simulating the grasping performance of a prosthetic hand, it seems practical to limit the GHs exploiting the human experience for grasping the objects, because in the actual use of the prosthesis the human brain will guide the arm motion to an adequate position and orientation of the wrist for the intended grasp. The use of human oriented approaches could solve some of these limitations. The selection of GHs inspired by the human hand was used in León et al. (León et al., 2013) to compare GQM obtained with the Michelangelo hand and with the biomechanical model of the human hand included in OpenHand. The use of grasp planning based on human demonstration has also allowed to reduce both the feasible workspace and the search space in robotic applications (Lin and Sun, 2015). Finally, the use of grasping simulation for prosthetic design requires a better definition of the outcome parameters. The adaptation of metrics based on experimental benchmarks for anthropomorphic hands could be an alternative. 
The final aim of this study is to present a preliminary approach to a new methodology for evaluating anthropomorphic prosthetic hands based on grasping simulation and assessment within the framework of the OpenRAVE simulation tool. This methodology involves the use of human knowledge for the generation of efficient GHs and the definition of a new metric to assess human likeness of the achievable grasps with the artificial hand. To this end, we propose to adapt to a simulation environment the Anthropomorphic Hand Assessment Protocol (AHAP) (Llop-Harillo et al., 2019), an experimental benchmark for grasping ability that considers the most common grasp types (GTs) during ADLs using everyday objects. To exemplify the methodology a comparison of thirty different DAs for the IMMA hand, an anthropomorphic prosthetic hand developed by the authors (Llop-Harillo and Pérez-González, 2017a), will be performed. Only the two most frequently used GTs included in the AHAP will be included in this preliminary approach, although the methodology can be easily extended including the eight main GTs included in the AHAP. The results obtained using this new benchmark will be compared with the results obtained using the brute-force approach and the GQM proposed in previous works and included in OpenHand (León et al., 2014, 2012; Rubert et al., 2017; Rubert and Morales, 2016). This comparison has the aim of analyzing whether the grasps generated with the brute-force approach that show good GQM correspond to realistic grasps in a human environment.

\section{Methods}

\subsection{IMMA Hand design alternatives}

The IMMA hand is a tendon-driven prosthetic hand prototype designed by the authors (Llop-Harillo and Pérez-González, 2017a). The original design of this hand, which will be considered as the baseline in this study, is publically available at (Llop-Harillo and Pérez-González, 2017b). It has six independently actuated degrees of freedom (DoFs): one for the flexion of each long finger, and two in the thumb, for flexion and circumduction movements.

In order to improve its mechanical design, different kinematic chain configurations have been analyzed in this study. The combination of the orientation angles of the carpometacarpal (CMC) joint and the metacarpophalangeal (MCP) joint of the thumb, allows the opposition of the thumb to orient its distal phalanx to the distal phalanges of the different long 


\section{6 | Chapter 7}

fingers. Therefore, with the aim of analyzing the best combination of these orientation angles to perform the most relevant GTs in an anthropomorphic way, several DAs have been proposed modifying these joint orientations. Moreover, two additional DAs have been proposed to analyze the effect of keeping fixed the distal interphalangeal (DIP) joints of the long fingers and the effect of adding the DoF of abduction in the MCP joints of the long fingers. Altogether, thirty DAs have been analyzed: combinations of the thumb CMC joint orientation $\left(0^{\circ}, 15^{\circ}, 30^{\circ}\right.$ and $45^{\circ}$ with respect to the proximal-distal axis) and the thumb MCP joint orientation $\left(0^{\circ}, 15^{\circ}, 30^{\circ}, 45^{\circ}\right.$, $60^{\circ}, 75^{\circ}$ and $90^{\circ}$ with respect to the CMC axis); thumb configuration of the baseline (CMC $0^{\circ}$ and MCP $45^{\circ}$ ) and DIP joints of the long fingers at a fixed flexion of $20^{\circ}$ (Belter et al., 2013) (named, DIP-fixed); thumb configuration of the baseline and abduction DoF of the MCP joints of the long fingers with a range of $15^{\circ}$ (Gracia-Ibáñez et al., 2017; Kapandji, 1982) (named, ABD): index and middle fingers with radial deviation and ring and little fingers with ulnar deviation. Figure 1 shows some of these DAs.

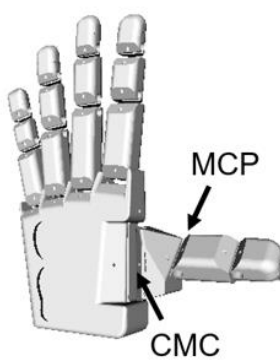

$\mathrm{CMC} 0^{\circ} \mathrm{MCP} 45^{\circ}$ (Baseline)

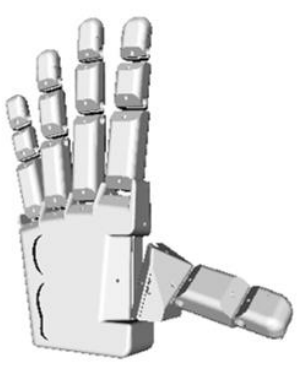

$\mathrm{CMC} 15^{\circ} \mathrm{MCP} 45^{\circ}$

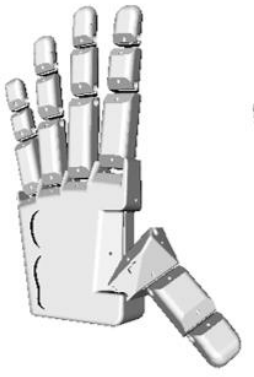

$\mathrm{CMC} 45^{\circ} \mathrm{MCP} 45^{\circ}$

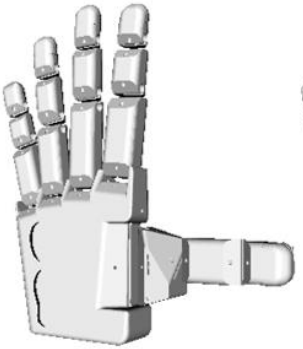

$\mathrm{CMC} 0^{\circ} \mathrm{MCP} 0^{\circ}$

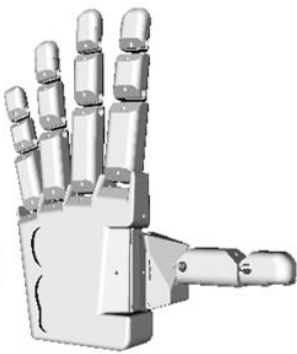

$\mathrm{CMC} 0^{\circ} \mathrm{MCP} 90^{\circ}$

Figure 1. Some of the thirty IMMA hand design alternatives. Thumb carpometacarpal (CMC) and metacarpophalangeal (MCP) joints orientation indicated.

\subsection{Grasp types and objects}

The AHAP (Llop-Harillo et al., 2019) is an experimental benchmark composed on 26 tasks involving grasping with the eight most relevant human GTs during ADLs and two non-grasping postures. It quantifies the grasping ability of anthropomorphic artificial hands, including the human-likeness and the grasp stability. The objects of the AHAP were selected from the Yale-CMU-Berkeley (YCB) set (Calli et al., 2015) due to their public availability. The objects selected account for variations in size and shape. For the simulation analysis in this paper, the objects were modelled in SolidWorks by the authors (Pérez-González and Llop-Harillo, 2019). For the 
sake of simplicity, only the prehensile GTs with a frequency of use above $10 \%$ according to Vergara et al. (Vergara et al., 2014) were used in this preliminary analysis: pulp pinch (PP) (38.3\%) and cylindrical grip (CG) (12.3\%). Figure 2 shows the six objects used in AHAP for the selected GTs and their approximate final position/orientation with respect to the artificial hand (Llop-Harillo et al., 2019).

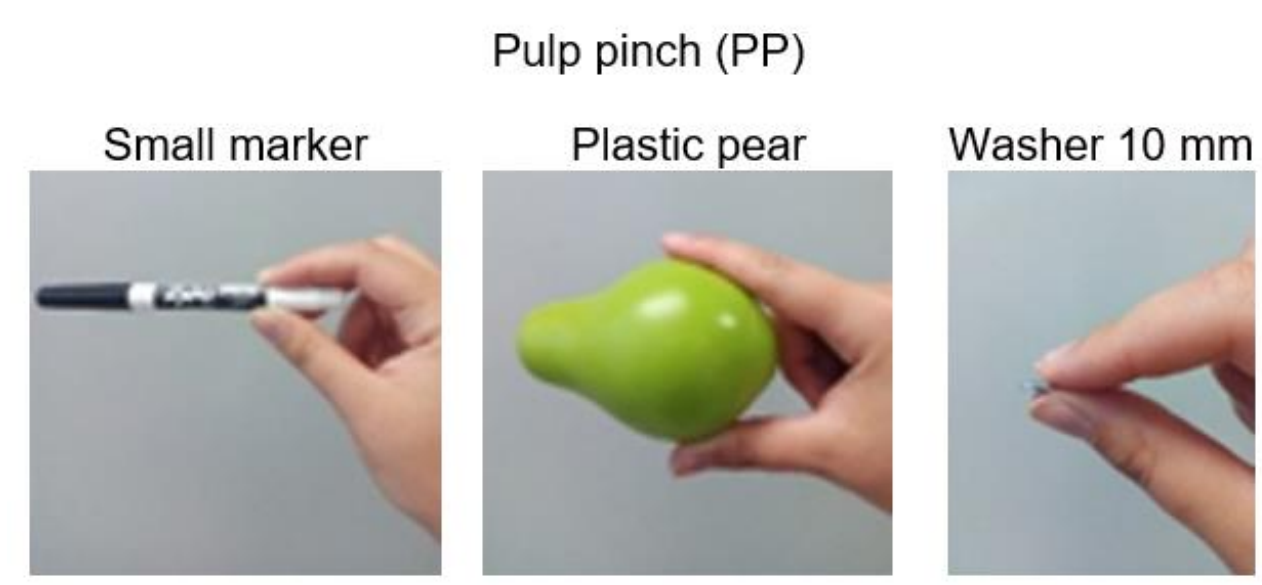

Cylindrical grip (CG)
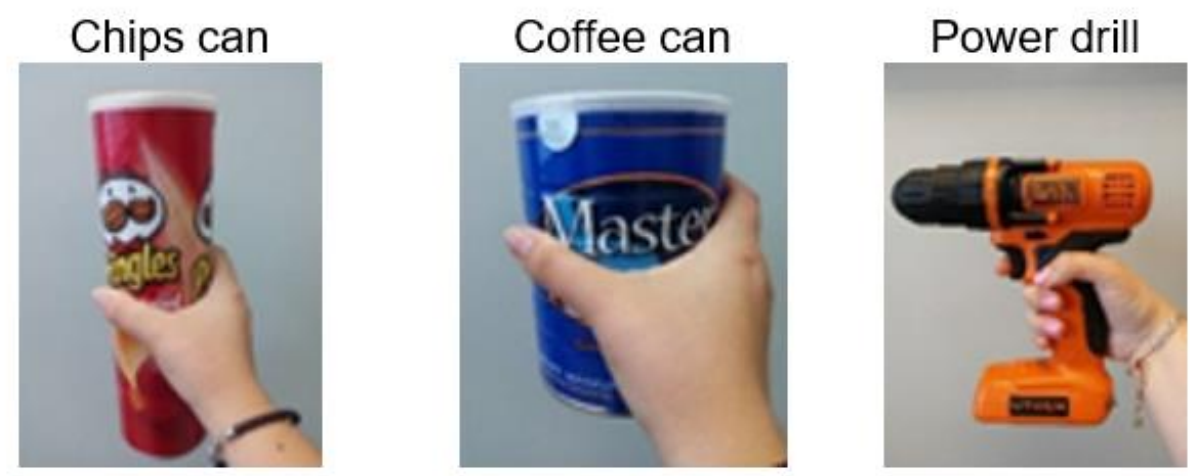

Figure 2. Grasp types and objects selected from the AHAP (Llop-Harillo et al., 2019).

\subsection{Grasp simulation: brute-force approach}

The brute-force approach for grasping simulation is based on the Grasping Module from the Database Generators available in OpenRAVE. The GHs are generated based on the following parameters:

- Distance between the points in the cartesian grid of the bounding box (d).

- Apex angle of the cone containing the approach rays $(\alpha)$. The cone axis is defined by the normal to the reference point in the object. 
- Standoff distance at which the palm of the hand stands still and the grip begins $(\delta)$.

- Angle to rotate the hand (roll) about the approach ray $(\theta)$.

In this study, the default parameters of OpenRAVE (Table 1) were used to generate the GHs. We used the ten GQM implemented in OpenHand (León et al., 2014; Rubert et al., 2017) to evaluate the grasps performed with the thirty IMMA hand DAs on the six objects selected above. The same procedure was repeated for each hand and object:

1. The Grasping Module is used to generate a variety of GHs using the parameters in Table 1.

2. For each GH, only the most proximal hand joint of every finger closes with a common velocity until the contact with the object is detected or the joint limit is reached. The process is repeated in the same way only for the joints located distally to the contacts. A final grasping posture is reached and the contact points are obtained.

3. The grasping posture is considered stable and defined as a successful grasp if the force-closure condition is accomplished.

4. The GQM are computed for up to 100 randomly selected successful grasps per object.

Table 1. Parameters selected to generate grasp hypotheses.

\begin{tabular}{cc}
\hline Parameter & Value \\
\hline $\mathrm{d}(\mathrm{m})$ & 0.02 \\
$\alpha(\mathrm{rad})$ & 0 \\
$\delta(\mathrm{m})$ & {$[0,0.025]$} \\
$\theta(\mathrm{rad})$ & {$[0, \pi / 2, \pi, 3 \pi / 2,2 \pi]$} \\
\hline
\end{tabular}

In order to analyze the grasps generated, some postprocessing was performed in Matlab. The hand DAs performing grasping postures for each object with the best value for each of the ten GQM were identified. The grasps of the hands performing better according to these GQM were analyzed. 


\subsection{Grasp simulation: Anthropomorphic grasps approach}

\subsubsection{Generation of grasp hypotheses}

For the new methodology proposed, all the simulations were conducted within the OpenRAVE tool. For this approach the OpenRAVE Grasping Module was extended by the authors with python scripting. The new extension allows the generation of a set of GHs with greater control than that of brute-force. These GHs are generated in such a manner that both the relative orientation and relative position of the hand/object in the approaching resemble the natural ones of the human hand. Likewise, the closure of the fingers (step 2 above) varies depending on the GT expected in the human case: all fingers close for CG, but only the thumb and index fingers are considered for PP. The grasps are evaluated with the force-closure condition (as in step 3 above).

For the grasp simulation a set of different GHs has been generated for each object. The GHs are based on the relative position of the object and the palm (common for all hand DAs). In addition to those defined in section 2.3, new parameters are needed for this approach (see Figures 3 and 4):

- $\mathrm{N}_{\alpha}$ : Number of approach rays distributed inside the cone with angle $\alpha$

- $\quad P$ : Approach reference point in the hand

- $\boldsymbol{n}$ : Approach direction vector in the hand

- $\theta_{\mathrm{i}}$ : Initial roll angle with respect vector $\mathbf{n}$

- $\theta_{\mathrm{f}}$ : Final roll angle with respect vector $\mathbf{n}$

- $\mathrm{N}_{\theta}$ : Number of rolls uniformly distributed between $\theta_{\mathrm{i}}$ and $\theta_{\mathrm{f}}$

For each object only one "target point" is considered in order to generate the approach rays. In Figure 3 the approach rays for each object are shown represented as red lines. These sets of rays are defined by two parameters: $\alpha$ and $\mathrm{N}_{\alpha}$.

In order to define the relative position between the object and the palm, a reference point $P$ and a vector $\boldsymbol{n}$ (with origin in the point $P$ ) are established in the hand (see Figure 4). For each ray in the object, the hand approaches toward the object aligning $\boldsymbol{n}$ and the ray. The point $P$ is the same for all the approaches and its coordinates defined in the local coordinates system of the palm are $x=10 \mathrm{~mm}, y=70 \mathrm{~mm}, z=0 \mathrm{~mm}$. The vector $\boldsymbol{n}$ is different depending on the object to be grasped, and has been chosen to imitate the natural 
approach of the human hand. Moreover, for the CG, the palm approaches toward the object till the contact is reached, but for the PP the fingers of the hand close while the palm remains at a certain standoff distance, imitating the natural human grasp. Table 2 shows all the parameter values selected for generating the GHs. The last column shows the total number of GHs tested per object $\left(\mathrm{N}_{\mathrm{GH}}=\mathrm{N}_{\alpha} \cdot \mathrm{N}_{\text {standoff }} \cdot \mathrm{N}_{\theta}\right.$ where $\mathrm{N}_{\text {standoff }}$ is the number of standoff distances considered, three for PP and one for CG).
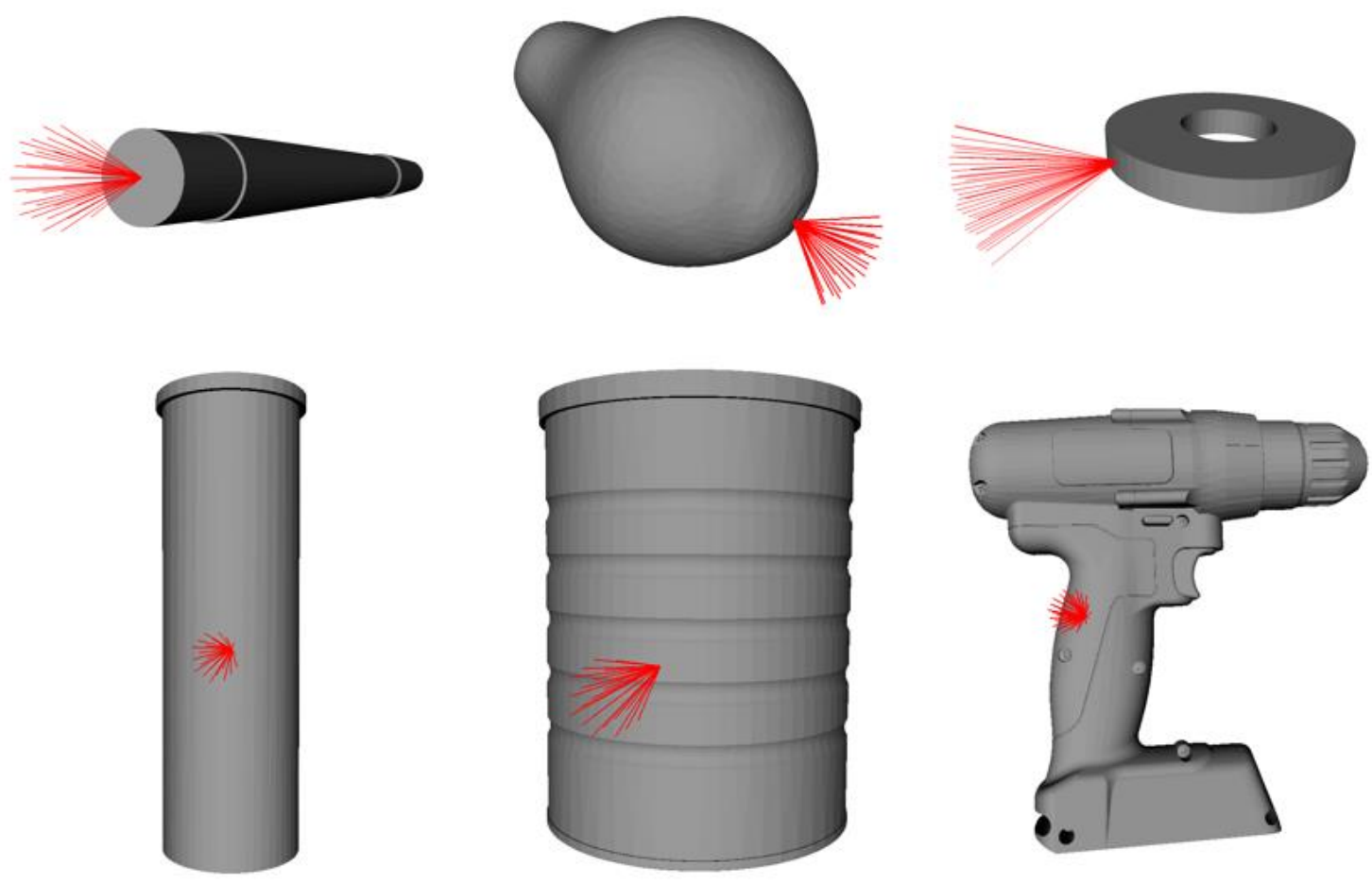

Figure 3. Approach rays considered for each object.

Table 2. Parameters selected to generate grasp hypotheses.

\begin{tabular}{cccccccc}
\hline Object & $\alpha(\mathrm{rad})$ & $\mathrm{N}_{\alpha}$ & $\boldsymbol{n}^{*}$ & $\delta(\mathrm{mm})$ & {$[\theta \mathrm{i}, \theta \mathrm{f}]$} & $\mathrm{N}_{\theta}$ & $\mathrm{N}_{\mathrm{GH}}$ \\
\hline Small marker & 0.45 & 38 & $(1,0,0)$ & $\{45,50,55\}$ & {$\left[-35^{\circ}, 35^{\circ}\right]$} & 9 & 1026 \\
Plastic pear & 0.8 & 24 & $(0.8,0,0.36)$ & $\{15,20,25\}$ & {$\left[-35^{\circ}, 35^{\circ}\right]$} & 9 & 648 \\
Washer & 0.45 & 38 & $(1,0,0)$ & $\{45,50,55\}$ & {$\left[-35^{\circ}, 35^{\circ}\right]$} & 9 & 1026 \\
Chips can & 0.2 & 28 & $(0.8,0,0.36)$ & $\{0\}$ & {$\left[-20^{\circ},-50^{\circ}\right]$} & 15 & 420 \\
Coffee can & 0.2 & 28 & $(0.8,0,0.36)$ & $\{0\}$ & {$\left[-20^{\circ},-50^{\circ}\right]$} & 15 & 420 \\
Power drill & 0.5 & 48 & $(0.8,0,0.36)$ & $\{0\}$ & {$\left[-15^{\circ}, 25^{\circ}\right]$} & 15 & 720 \\
\hline
\end{tabular}

*n is defined in the local coordinates system of the palm 


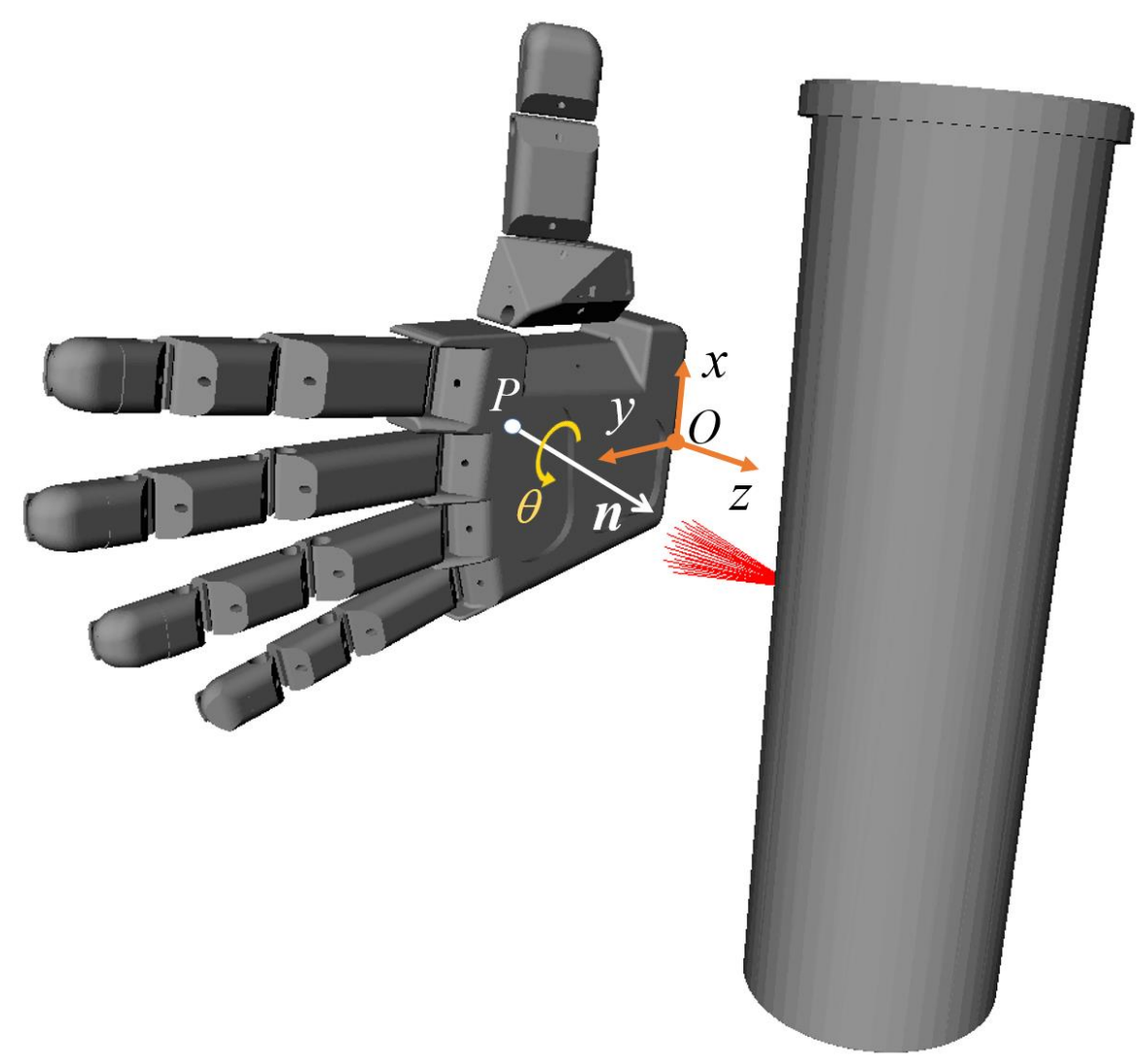

Figure 4. Approach parameters and local coordinates system of the palm.

\subsubsection{Evaluation of anthropomorphic grasp correctness}

We propose to evaluate the grasping postures following the same anthropomorphic criteria considered in the AHAP (GT correctness). For each final grasping posture, the developed OpenRAVE extension script is able to automatically evaluate whether the conditions are met or not for an anthropomorphic CG or PP, according to the conditions established in the AHAP (Llop-Harillo et al., 2019): "PP: The GT is considered correct if the object contacts with the palmar sides of the distal phalange of the thumb and the distal phalange of only one long finger, without any contact of the object with the palm; CG: The GT is considered correct if the angle between the main axis of the thumb and the main axis of the object's grip area is greater than $60^{\circ}$ and there is contact between the object and the palmar sides of the thumb, all the phalanges of at least three long fingers and the palm".

To check these conditions, a contact is considered to happen in the palmar side of a phalanx if the angle between the normal contact at that point and the palmar normal vector in this phalanx ( $\boldsymbol{n}_{\text {palmar }}$ in Figure 5 ) is less than $75^{\circ}$. The thumb main axis direction is defined by a vector $\boldsymbol{n}_{\text {main }}$ shown in Figure 5. For the three CG objects, the main axis of the object's grip area is vertical in Figure 3. 


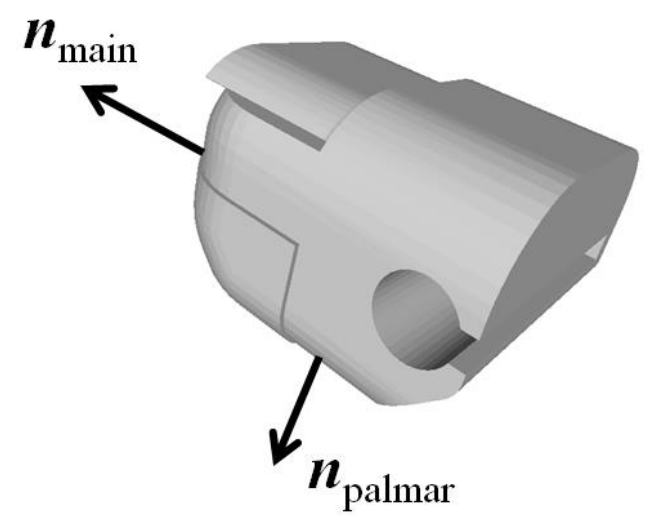

Figure 5. Thumb distal phalanx showing the orientation of the main axis of the thumb and the palmar normal of the fingers phalanges.

\subsubsection{Simulated Grasping Ability Score}

In order to compare the human-like grasping and the stability of the grasps performed with the different hand DAs a new index is proposed, adapted from the Grasping Ability Score (GAS) defined in the AHAP (LlopHarillo et al., 2019). Equation 1 shows the proposed index, named Simulated Grasping Ability Score ( $\left.\mathrm{S}_{\mathrm{GAS}}\right)$, for each object:

$$
S_{G A S_{i}}=\frac{1}{N_{G H_{i}}}\left[C_{i}+0.5 \cdot N C_{i}\right]
$$

where $i$ refers to the object $(i=1, \ldots 6) ; N_{G H i}$ is the total number of GHs tested for object $i$ (defined in section 2.4.1); and $C_{i}$ and $N C_{i}$ are, respectively, the number of correct grasps and not correct grasps, according to the GT correctness criteria of the AHAP (section 2.4.2), on object $i$ among those satisfying the force-closure condition. The factor 0.5 weights with half score the force-closure grasps that do not are anthropomorphic according to the GT correctness criteria, while the grasps accomplishing both force-closure and GT correctness score 1 point, similarly to the GAS. The $\mathrm{S}_{\mathrm{GAS}}$ defined in this way ranges between 0 and 1 .

In this study, the index has been obtained for the six different objects selected. Moreover, an index per GT has also been obtained (Equation 2 with $n=3$ ), averaging the index of the different objects considered for each GT, and a total index has been obtained averaging the index of all the objects (Equation 2 with $n=6$ ).

$$
S_{G A S}=\frac{1}{n} \sum_{i=1}^{n} S_{G A S_{i}}
$$




\section{Results}

\subsection{Brute-force approach}

Table 3 shows, for each object, the IMMA hand DA that performed the best grasp according to each of the GQM included in OpenHand. The washer does not appear in this table because none of the grasps performed with this object was a successful grasp according to the force-closure condition. The ABD DA performed most of those grasps (12/50). Followed by the DA with thumb CMC $15^{\circ}$ and MCP $90^{\circ}$ (6/50). The rest of DAs appear less than three times. Figures 6 and 7 show the grasps with the best GQM performed by these two best hand designs. It can be seen that most of the grasps do not resemble those shown in Figure 2. In particular, none of the grasps for the cylindrical GT objects is really a CG and the grasp postures are weird from a human perspective. Something similar occurs with the PP objects.

Table 3. Hand designs that performed the grasps with the best GQM for each object.

\begin{tabular}{lcccccccccc}
\hline & $\mathrm{Q}_{\mathrm{A} 1}$ & $\mathrm{Q}_{\mathrm{A} 2}$ & $\mathrm{Q}_{\mathrm{A}}$ & $\mathrm{Q}_{\mathrm{B} 1}$ & $\mathrm{QB}_{\mathrm{B}}$ & $\mathrm{Q}_{\mathrm{B} 3}$ & $\mathrm{Q}_{\mathrm{C} 1}$ & $\mathrm{Q}_{\mathrm{C} 2}$ & $\mathrm{Q}_{\mathrm{D} 1}$ & $\mathrm{Q}_{\mathrm{D} 2}$ \\
\hline $\begin{array}{l}\text { Small } \\
\text { marker }\end{array}$ & CMC 30 & CMC 15 & CMC 30 & ABD & CMC 15 & CMC 15 & CMC 30 & CMC 45 & ABD & CMC 15 \\
Plastic & CMC 30 & ABD & CMC 30 & CMC 15 & CMC 30 & ABD & CMC 45 & ABD & CMC 30 & CMC 15 \\
pear & MCP 30 & & MCP 30 & MCP 75 & MCP 75 & & MCP 45 & & MCP 60 & MCP 0 \\
Chips & CMC 30 & CMC 30 & ABD & CMC 45 & CMC 30 & CMC 15 & ABD & ABD & CMC 30 & CMC 30 \\
can & MCP 15 & MCP 15 & & MCP 45 & MCP 15 & MCP 90 & & & MCP 60 & MCP 0 \\
Coffee & ABD & CMC 30 & CMC 0 & CMC 45 & CMC 45 & CMC 0 & CMC 0 & ABD & CMC 30 & ABD \\
can & & MCP 45 & MCP 90 & MCP 45 & MCP 15 & MCP 60 & MCP 30 & & MCP 30 \\
$\begin{array}{l}\text { Power } \\
\text { drill }\end{array}$ & CMC 45 & CMC 45 & CMC 0 & CMC 0 & CMC 45 & ABD & CMC 15 & CMC 45 & CMC 15 & CMC 15 \\
\hline
\end{tabular}
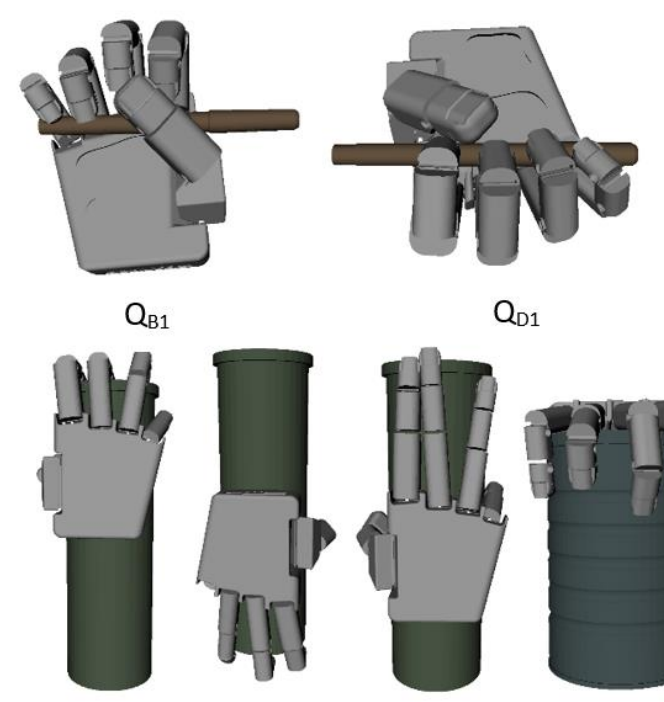

$\mathrm{Q}_{\mathrm{A3}}$

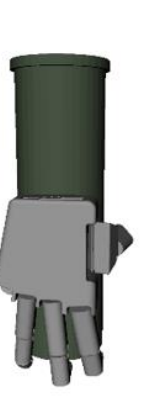

$\mathrm{Q}_{\mathrm{c1}}$
$\mathrm{Q}_{\mathrm{D} 1}$

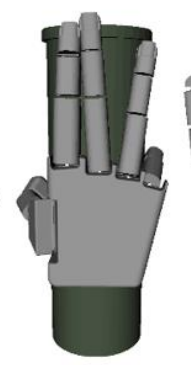

$\mathrm{Q}_{\mathrm{C2}}$

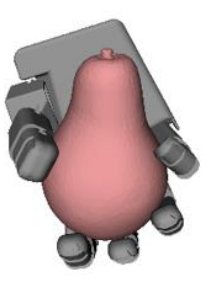

$\mathrm{Q}_{\mathrm{A2}}$

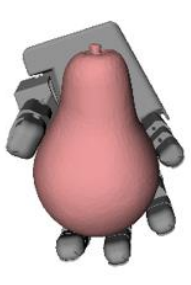

$\mathrm{Q}_{\mathrm{B} 3}$

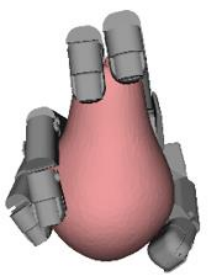

$\mathrm{Q}_{\mathrm{c2}}$

Figure 6. Grasps with the best GQM performed by the ABD design alternative of the IMMA hand. 


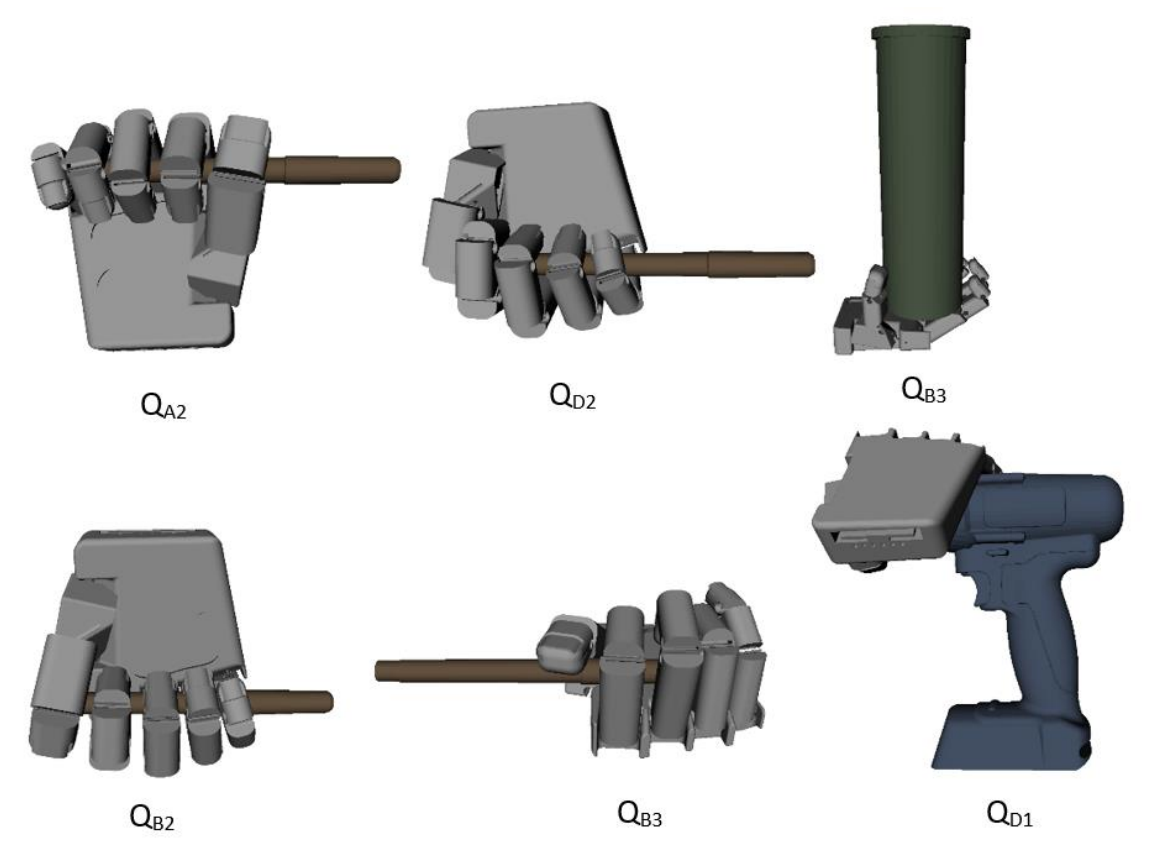

Figure 7. Grasps with the best GQM performed by the IMMA hand design alternative with thumb CMC $15^{\circ}$ and MCP $90^{\circ}$.

\subsection{Anthropomorphic grasps approach}

Figure 8 shows the results of total $\mathrm{S}_{\mathrm{GAS}}$ versus MCP angle for each CMC angle. Among the hand DAs analyzed changing CMC and MCP thumb angles, the best total $\mathrm{S}_{\mathrm{GAS}}$ (44 \%) was obtained for that with CMC $45^{\circ}$ and MCP $60^{\circ}$. For each CMC angle, the optimal MCP angle is different, being smaller for bigger CMC angles. Figure 9 shows a 2D contour plot representation of the results obtained for total $\mathrm{S}_{\mathrm{GAS}}$ by spline interpolation (grid spacing $1^{\circ}$ ) from the computed DAs. The optimal design according to the total $\mathrm{S}_{\mathrm{GAS}}$ is close to CMC $40^{\circ}-45^{\circ}$ and MCP $45^{\circ}-60^{\circ}$. The baseline obtained a total $\mathrm{S}_{\mathrm{GAS}}$ of $25 \%$ and the DA DIP-fixed $16 \%$, the worst value among the DAs analyzed. The ABD DA scored $24 \%$ on $\mathrm{S}_{\mathrm{GAS}}$, indicating that the addition of the abduction DoF on the MCP joints of the long fingers did not improve the baseline.

Figure 10 shows the mean and standard deviation across hand DAs of the number of grasps that accomplish the force-closure condition (FC) and the number of grasps that accomplish both the force-closure condition and the GT correctness (C), for each object analyzed along with the number of tested GHs $\left(\mathrm{N}_{\mathrm{GH}}\right)$. It is worth noting that $\mathrm{NC}$ in Equation 1 can be obtained as the difference between FC and C. For the PP objects (Small marker, Plastic Pear, Washer) the difference between FC and C is small. However, for the CG objects (Chips can, Coffee can, Power drill) this difference is really high because the number of correct grasps is very low. Notwithstanding, the 
force-closure condition is obtained in a greater fraction of the tested GH for CG. As an example, Figure 11 shows a CG that accomplish force-closure condition but not the GT correctness criteria because one phalanx of the ring and two phalanges of the little finger are not contacting the object. Figure 12 shows, for the best hand DA, one of the grasps accomplishing GT correctness for each object.

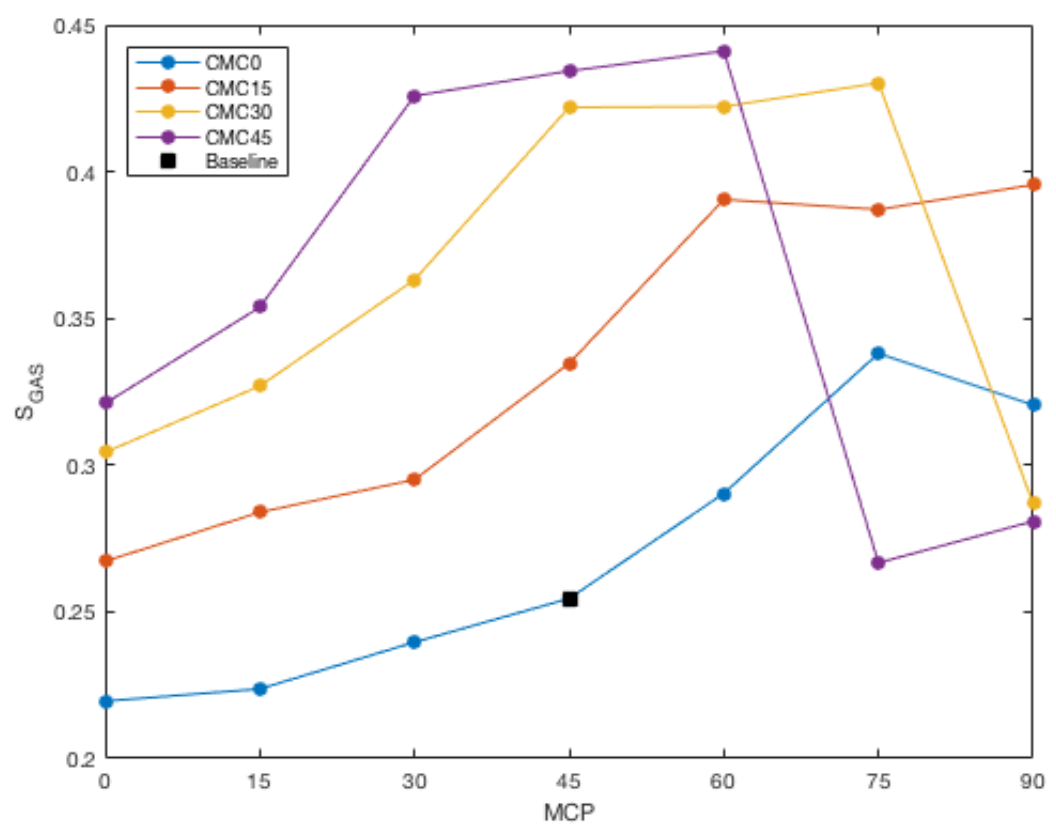

Figure 8. Total $\mathrm{S}_{\mathrm{GAS}}$ as a function of MCP angle for each $\mathrm{CMC}$ angle.

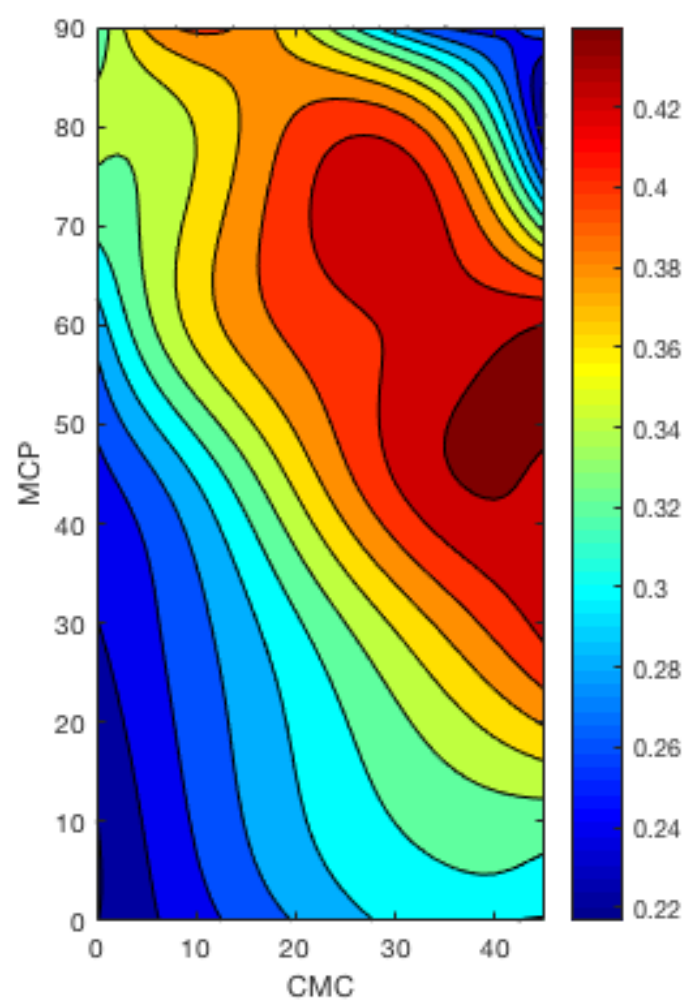

Figure 9. Total $\mathrm{S}_{\mathrm{GAS}}$ as a function of $\mathrm{CMC}$ and MCP angles, obtained by spline interpolation (grid spacing $1^{\circ}$ ) from the computed design alternatives. 


\section{6 | Chapter 7}

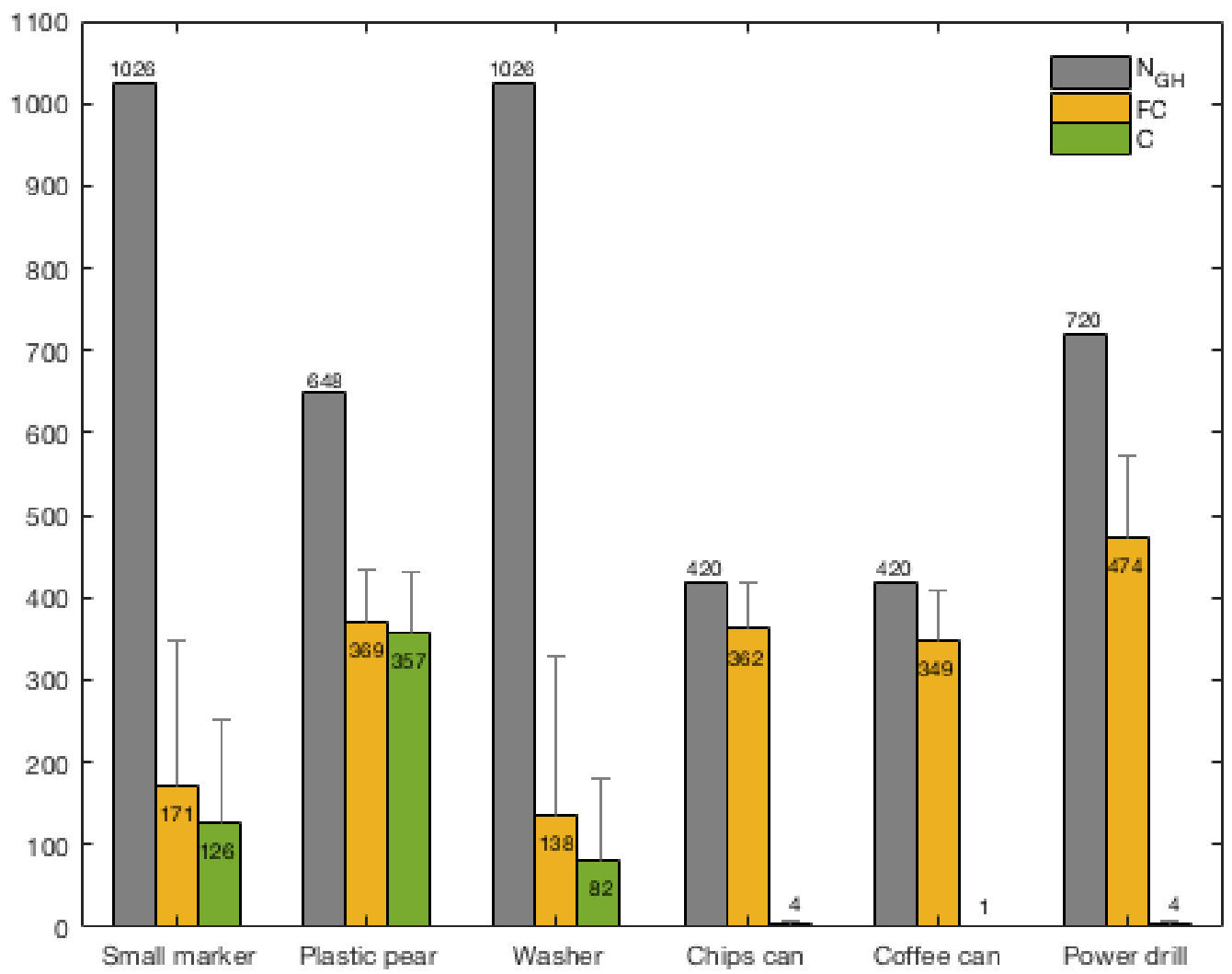

Figure 10. Mean and standard deviation of force-closure grasps (FC) and correct grasps (C) across hand design alternatives for each object.

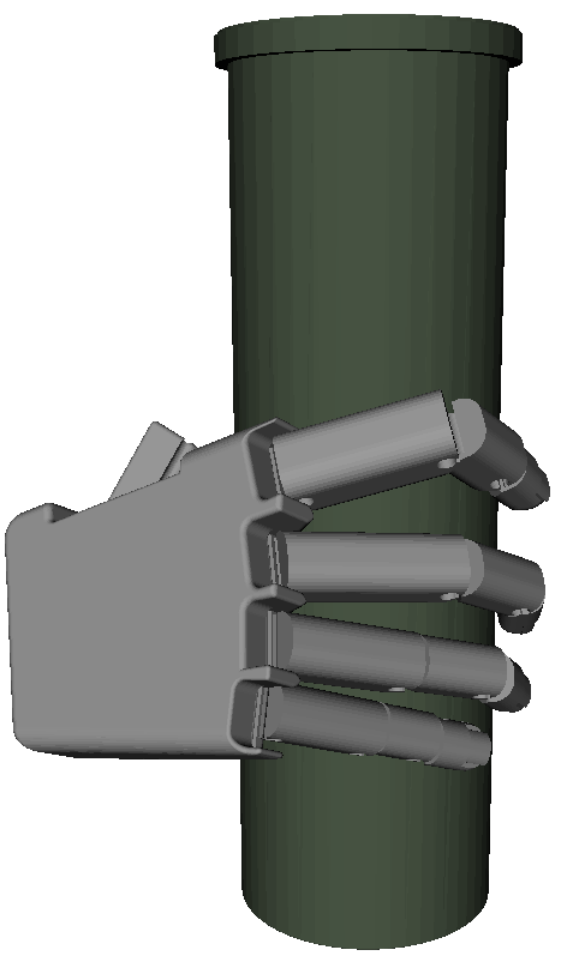

Figure 11. Cylindrical grip that seems anthropomorphic but does not satisfy the grasp type correctness criteria of the AHAP. 


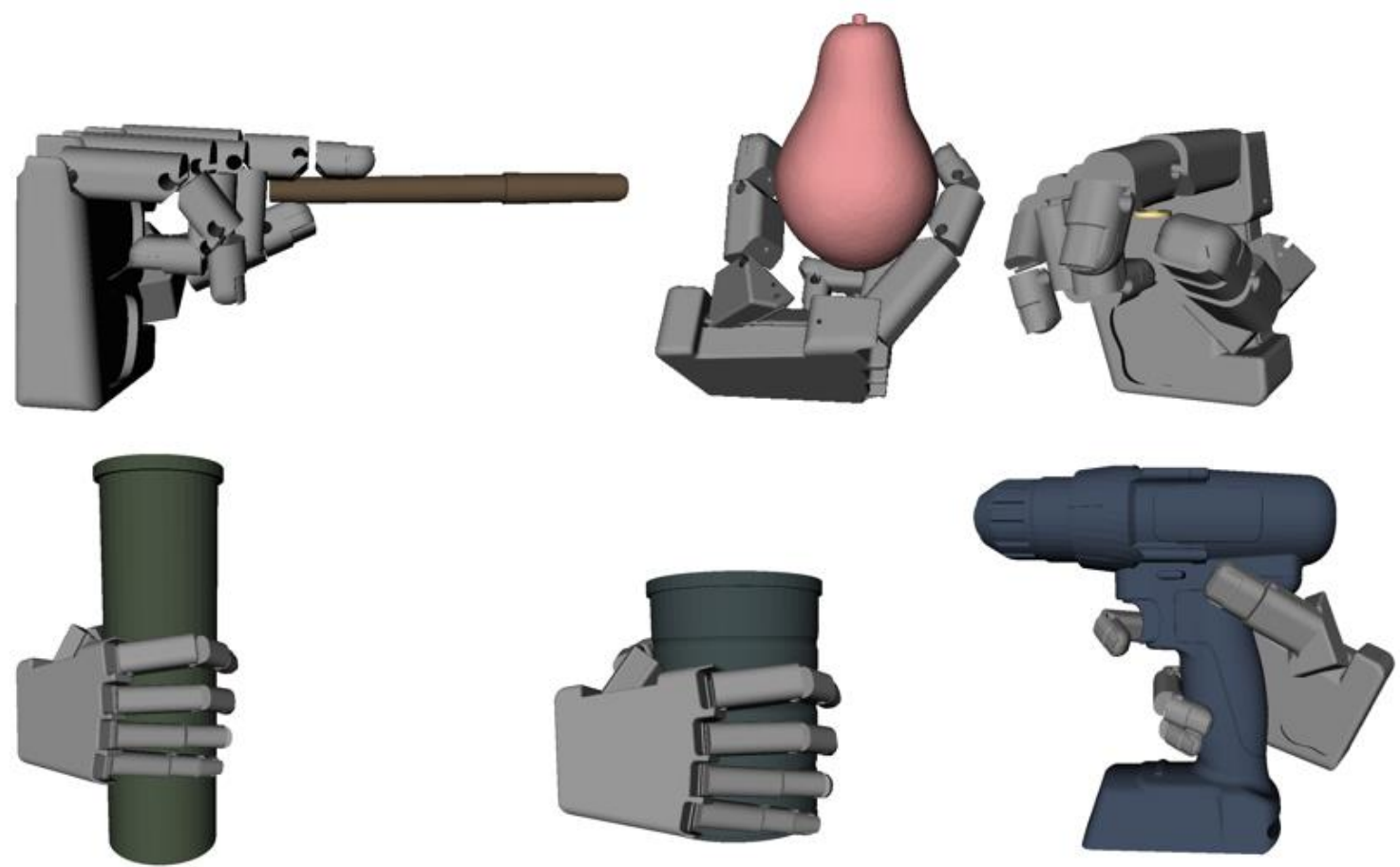

Figure 12. Correct grasps performed with the IMMA hand design alternative with thumb CMC $45^{\circ}$ and MCP $60^{\circ}$.

\section{Discussion}

This study is focused on assessing and comparing prosthetic hand designs according to their anthropomorphism on the simulated grasps performed. According to our results, the brute-force approach and the evaluation with GQM have evident limitations for evaluating grasping anthropomorphism of artificial hands for the main GTs. Figures 6 and 7 show that some of the best grasps selected with this approach do not fulfill the criteria of anthropomorphism defined in the literature (Llop-Harillo et al., 2019; Sollerman and Ejeskär, 1995; Vergara et al., 2014) for the expected GTs (PP and CG). Moreover, the majority of the grasps obtained with this approach are not realistic for the expected use of the objects by a human in ADLs. The preliminary benchmark proposed in this study allows undertaking the comparison of hand DAs based on anthropomorphic criteria apart from being more efficient in terms of computation time. The final grasps obtained with this approach are more meaningful in terms of anthropomorphism and ADLs (see Figure 12). The fact that the position of the target point for the GHs is unique in the procedure is not a relevant limitation, because in prosthetic hands the users select the approaching point for the grasp. The different GHs generated around this target point evaluate the robustness of each DA for achieving the correct grasp under slight variations in orientation or position. 


\section{8 | Chapter 7}

A noteworthy advantage of the simulation benchmark proposed is the reduced time required to compare different hand DAs against the experimental approach. In this study, twenty-eight different DAs of the thumb CMC and MCP joint orientations have been analyzed easily. An experimental comparison for such a big number of alternatives would be unaffordable in a reasonable time.

Among the DAs tested here for the IMMA hand, the best result on $\mathrm{S}_{\mathrm{GAS}}$ was obtained using CMC $45^{\circ}$ and MCP $60^{\circ}$. Figure 8 shows that DAs with CMC $45^{\circ}$ and MCP between $30^{\circ}$ and $60^{\circ}$, and those with CMC $30^{\circ}$ and MCP between $45^{\circ}$ and $75^{\circ}$ obtained similar values on total $\mathrm{S}_{\mathrm{GAs}}$. It is worth noting that for these DAs the sum of CMC and MCP angles ranges between $75^{\circ}$ and $105^{\circ}$. These orientations allow a good opposition between the thumb and index fingers needed to perform PP as well as a good orientation of the thumb with respect to the main axis of the object grip area, needed to perform correctly CG. Other thumb joint orientations with a lower or higher sum of angles are not so suitable to perform these anthropomorphic grasps. Figure 9 shows this trend because the highest scores are found in a fringe where CMC and MCP orientation angles sum around $90^{\circ}-100^{\circ}$. A deeper analysis of the results, not shown here for brevity but available as Supplementary Material, indicates that for some hand DAs, located in the bottom-left and top-right corners of Figure 9, the $\mathrm{S}_{\mathrm{GAS}}$ is even null for the PP with the small marker and very low for the washer, because the opposition of the thumb to the index finger does not allow to reach the force-closure condition.

The detailed results of $\mathrm{S}_{\mathrm{GAS}}$ obtained with the baseline and the DAs DIPfixed and ABD show that the DIP-fixed worsened the stability and anthropomorphism of the grasps while the ABD DA performed worse PP and mostly better CG than the baseline. The difference in total $\mathrm{S}_{\mathrm{GAS}}$ between the baseline (25\%) and ABD (24\%) is small. This result is unexpected because the addition of the abduction DoF should improve the ability of the hand to reach better postures. However, a limitation in the method used to move the different hand joints in the simulation framework could explain this result: the abduction is performed before the flexion of the long fingers. This chained motion can be counterproductive for a good opposition in PP.

In a previous work by the authors (Llop-Harillo et al., n.d.) the original IMMA hand (baseline) was tested on three able-bodied subjects performing experimentally the AHAP with independent actuation of each of its DoFs. The results obtained in that experimental study agree partially with those obtained in the present one. For the plastic pear and power drill it was 
possible to obtain correct grasps (satisfying GT correctness) with both approaches (see Supplementary Materials). For the small marker, a very low fraction $(2.5 \%)$ of correct grasps were obtained in the simulation and none experimentally over nine trials. Nevertheless, for the other objects some discrepancies appear. For the chips and coffee cans, some correct grasps were performed experimentally but none in the simulation among the tested GHs. Even if some of the final grasps obtained in the simulation seem to be CG (see Figure 11), they are not classified as correct, because the GT correctness criteria for CG requires the contact with the object of all the phalanges of at least three long fingers. The method used in OpenRAVE to obtain the final grasping posture could explain the problem to reach correct grasps for CG in the simulation, because the motion of a finger stops if the distal segment contact first with the object, making impossible in such cases the contact with the proximal phalanges. For the washer, no correct grasp was obtained with the baseline design in the simulation, despite it was possible experimentally. In this case, we think that the difference is also due to the method used in OpenRAVE to simulate the closing motion of the fingers, because the CMC joint of the thumb closes completely before the MCP joint begins to close, hampering the right opposition with the index finger necessary for force-closure and GT correctness. In contrast, the two DoFs of the thumb were controlled independently by different subject's fingers during the experiments (Llop-Harillo et al., n.d.). Therefore, we can conclude that some improvements in the simulation framework should be undertaken in order to improve its predictive performance.

Notwithstanding the limitations of the simulation analysis, the anthropomorphic approach proposed in this study offers a good benchmark to evaluate anthropomorphic grasps in order to select optimal hand DAs. This preliminary benchmark is based only on the two most frequent GTs, despite the experimental AHAP being composed of eight GTs. In the near future, we plan to include all these GTs in the simulated benchmark in order to make the assessment of hand DAs more representative of ADLs.

\section{Conclusion}

In this study, two different simulation approaches focused on assessing grasping in prosthetic hand designs have been compared with the aim of obtaining a useful metric to measure their anthropomorphism and functionality during ADLs. The combination of the brute-force approach for generating GHs and GQM for the evaluation of the final grasping posture, 


\section{0 | Chapter 7}

used in previous works in the literature, resulted on neither anthropomorphic nor realistic grasps for the expected use of the objects by a human. The new simulation benchmark proposed follows an anthropomorphic approach for defining the GHs and for evaluating stability and human likeness. Its principles are adapted from the experimental benchmark AHAP and considers the GT correctness criteria according to human experience. The new approach resulted in more anthropomorphic and realistic grasps and together with the metric defined allows improving the comparison of different hand DAs. In a comparison of thirty different DAs of the IMMA hand changing mainly the orientation of the CMC and MCP joints of the thumb with respect to the baseline design, the new benchmark has allowed to define preferable orientations for these joints in order to improve significantly the number of successful and anthropomorphically correct grasps for PP and CG, the most frequent GTs. In the near future, this preliminary proposal will be completed including all the main GTs used in ADLs considered in the AHAP.

\section{Acknowledgements}

This work was supported by the Spanish Ministry of Economy and Competitiveness and ESF [grant number BES-2015-076005]; the Spanish Ministry of Economy and Competitiveness, AEI and ERDF [grant numbers DPI2014-60635-R, DPI2017-89910-R]; Universitat Jaume I, Spain [grant number UJI-B2017-70]; and Generalitat Valenciana (Spain) [grant number GV/2018/125].

We also acknowledge Carlos Rubert for his contribution in the integration of the hands in the simulation framework and in the simulations performed with OpenHand.

\section{References}

Belter, J.T., Segil, J.L., Dollar, A.M., Weir, R.F., 2013. Mechanical design and performance specifications of anthropomorphic prosthetic hands: A review. The Journal of Rehabilitation Research and Development, 50(5), 599-617.

Calli, B., Walsman, A., Singh, A., Srinivasa, S., Abbeel, P., Dollar, A.M., 2015. Benchmarking in Manipulation Research: Using the Yale-CMU-Berkeley Object and Model Set. IEEE Robotics \& Automation Magazine, 22(3), 36-52.

COLLADA, n.d. https://www.khronos.org/collada/

Diankov, R., 2010. Automated Construction of Robotic Manipulation Programs. 
Architecture, Ph.D., 1-263.

Falco, J., Van Wyk, K., Liu, S., Carpin, S., 2015. Grasping the Performance: Facilitating Replicable Performance Measures via Benchmarking and Standardized Methodologies. IEEE Robotics \& Automation Magazine, 22(4), 125-136.

Feix, T., Romero, J., Ek, C.H., Schmiedmayer, H.B., Kragic, D., 2013. A metric for comparing the anthropomorphic motion capability of artificial hands. IEEE Transactions on Robotics, 29(1), 82-93.

Gracia-Ibáñez, V., Vergara, M., Sancho-Bru, J.L., Mora, M.C., Piqueras, C., 2017. Functional range of motion of the hand joints in activities of the International Classification of Functioning, Disability and Health. Journal of Hand Therapy, 30(3), 337-347.

Huamán Quispe, A., Ben Amor, H., Christensen, H.I., 2018. A Taxonomy of Benchmark Tasks for Robot Manipulation, in: Springer Proceedings in Advanced Robotics. pp. 405-421.

Jang, C.H., Yang, H.S., Yang, H.E., Lee, S.Y., Kwon, J.W., Yun, B.D., Choi, J.Y., Kim, S.N., Jeong, H.W., 2011. A Survey on Activities of Daily Living and Occupations of Upper Extremity Amputees. Annals of Rehabilitation Medicine, 35(6), 907.

Kapandji, I.A., 1982. The Physiology of the Joints: Upper limb. Churchill Livingstone.

León, B., Morales, A., Sancho-Bru, J., 2014. From Robot to Human Grasping Simulation, Cognitive Systems Monographs, Cognitive Systems Monographs. Springer International Publishing, Cham.

León, B., Rubert, C., Sancho-Bru, J., Morales, A., 2013. Evaluation of prosthetic hands prehension using grasp quality measures, in: 2013 IEEE/RSJ International Conference on Intelligent Robots and Systems. IEEE, pp. 35013506.

León, B., Sancho-Bru, J.L., Jarque-Bou, N.J., Morales, A., Roa, M.A., 2012. Evaluation of human prehension using grasp quality measures. International Journal of Advanced Robotic Systems, 9.

León, B., Ulbrich, S., Diankov, R., Puche, G., Przybylski, M., Morales, A., Asfour, T., Moisio, S., Bohg, J., Kuffner, J., Dillmann, R., 2010. OpenGRASP: A Toolkit for Robot Grasping Simulation, in: Lecture Notes in Computer Science (Including Subseries Lecture Notes in Artificial Intelligence and Lecture Notes in Bioinformatics). pp. 109-120.

Levine, S., Pastor, P., Krizhevsky, A., Ibarz, J., Quillen, D., 2018. Learning handeye coordination for robotic grasping with deep learning and large-scale data collection. The International Journal of Robotics Research, 37(4-5), 421436. 


\section{2 | Chapter 7}

Liarokapis, M. V., Artemiadis, P.K., Kyriakopoulos, K.J., 2013. Quantifying anthropomorphism of robot hands, in: Proceedings - IEEE International Conference on Robotics and Automation. IEEE, pp. 2041-2046.

Lin, Y., Sun, Y., 2015. Robot grasp planning based on demonstrated grasp strategies. The International Journal of Robotics Research, 34(1), $26-42$.

Llop-Harillo, I., Pérez-González, A., 2017a. System for the experimental evaluation of anthropomorphic hands. Application to a new 3D-printed prosthetic hand prototype. International Biomechanics, 4(2), 50-59.

Llop-Harillo, I., Pérez-González, A., 2017b. IMMA hand (Devalhand project). https://sites.google.com/a/uji.es/devalhand/imma-hand

Llop-Harillo, I., Pérez-González, A., Andrés, F.J., n.d. Grasping ability and motion synergies in affordable tendon-driven prosthetic hands controlled by ablebodied subjects. Submitted to Frontiers in Neurorobotics.

Llop-Harillo, I., Pérez-González, A., Starke, J., Asfour, T., 2019. The Anthropomorphic Hand Assessment Protocol (AHAP). Robotics and Autonomous Systems, 121.

Miller, A.T., Allen, P.K., 2004. Graspit: A versatile simulator for robotic grasping. IEEE robotics automation magazine, 11(4), 110.

Morales, A., Chinellato, E., Fagg, A.H., Del Pobil, A.P., 2004. Using experience for assessing grasp reliability. International Journal of Humanoid Robotics, 1(4), 671-691.

Pérez-González and Llop-Harillo, 2019. 3D models of the objects selected (from YCB set) for the different grasp types in the Anthropomorphic Hand Assessment Protocol (AHAP).

Pinto, L., Gupta, A., 2016. Supersizing self-supervision: Learning to grasp from 50K tries and 700 robot hours, in: 2016 IEEE International Conference on Robotics and Automation (ICRA). IEEE, pp. 3406-3413.

Roa, M.A., Suárez, R., 2014. Grasp quality measures: review and performance. Autonomous Robots, 38(1), 65-88.

Rubert, C., León, B., Morales, A., Sancho-Bru, J., 2017. Characterisation of Grasp Quality Metrics. Journal of Intelligent \& Robotic Systems, 89(3-4), 319-342.

Rubert, C., Morales, A., 2016. Comparison between grasp quality metrics and the anthropomorphism index for the evaluation of artificial hands, in: 2016 6th IEEE International Conference on Biomedical Robotics and Biomechatronics (BioRob). IEEE, pp. 1352-1357.

Sahbani, A., El-Khoury, S., Bidaud, P., 2012. An overview of 3D object grasp synthesis algorithms. Robotics and Autonomous Systems, 60(3), 326-336.

Sollerman, C., Ejeskär, A., 1995. Sollerman Hand Function Test: A Standardised Method and its Use in Tetraplegic Patients. Scandinavian Journal of Plastic 
and Reconstructive Surgery and Hand Surgery, 29(2), 167-176.

Vergara, M., Sancho-Bru, J.L., Gracia-Ibáñez, V., Pérez-González, A., 2014. An introductory study of common grasps used by adults during performance of activities of daily living. Journal of hand therapy: official journal of the American Society of Hand Therapists, 27, 1-28.

\section{Supplementary Material}

\begin{tabular}{|c|c|c|c|c|c|c|c|c|c|c|c|c|c|c|c|c|c|c|c|c|c|c|}
\hline \multicolumn{2}{|c|}{ IMMA DAs } & \multicolumn{3}{|c|}{ Small marker } & \multicolumn{3}{|c|}{ Plastic pear } & \multicolumn{3}{|c|}{ Washer } & \multicolumn{3}{|c|}{ Chips can } & \multicolumn{3}{|c|}{ Coffee can } & \multicolumn{3}{|c|}{ Power drill } & \multirow{2}{*}{$\begin{array}{c}\text { PP } \\
\text { SGAS } \\
(\%)\end{array}$} & \multirow{2}{*}{$\begin{array}{c}\text { CG } \\
\text { SGAS } \\
(\%)\end{array}$} & \multirow{2}{*}{$\begin{array}{l}\text { Total } \\
\text { SGAS } \\
(\%)\end{array}$} \\
\hline $\begin{array}{c}\text { CMC } \\
\left(^{\circ}\right)\end{array}$ & $\begin{array}{c}\text { MCP } \\
\left(^{\circ}\right)\end{array}$ & $\begin{array}{l}\text { FC } \\
(\%)\end{array}$ & $\begin{array}{c}\mathrm{C} \\
(\%)\end{array}$ & $\begin{array}{l}\text { SGAS } \\
(\%)\end{array}$ & $\begin{array}{l}\text { FC } \\
(\%)\end{array}$ & $\begin{array}{c}C \\
(\%)\end{array}$ & $\begin{array}{l}\text { SGAS } \\
(\%)\end{array}$ & $\begin{array}{l}\text { FC } \\
(\%)\end{array}$ & $\begin{array}{c}C \\
(\%)\end{array}$ & $\begin{array}{l}\text { SGAS } \\
(\%)\end{array}$ & $\begin{array}{l}\text { FC } \\
(\%)\end{array}$ & $\begin{array}{c}\mathrm{C} \\
(\%)\end{array}$ & $\begin{array}{l}\text { SGAS }_{\text {GA) }} \\
(\%)\end{array}$ & $\begin{array}{l}\text { FC } \\
(\%)\end{array}$ & $\begin{array}{c}\mathrm{C} \\
(\%)\end{array}$ & $\begin{array}{l}\text { SGAS } \\
(\%)\end{array}$ & $\begin{array}{l}\text { FC } \\
(\%)\end{array}$ & $\begin{array}{c}\mathrm{C} \\
(\%)\end{array}$ & $\begin{array}{l}\text { SGAAS }_{\text {(\%) }} \\
(\%)\end{array}$ & & & \\
\hline 0 & 0 & 0.0 & 0.0 & 0.0 & 31.8 & 30.2 & 31.0 & 0.4 & 0.0 & 0.2 & 66.2 & 0.0 & 33.1 & 61.7 & 0.0 & 30.8 & 72.8 & 0.4 & 36.6 & 10.4 & 33.5 & 22.0 \\
\hline 0 & 15 & 0.0 & 0.0 & 0.0 & 36.7 & 34.4 & 35.6 & 0.4 & 0.0 & 0.2 & 63.3 & 0.0 & 31.7 & 56.7 & 0.0 & 28.3 & 76.5 & 0.4 & 38.5 & 11.9 & 32.8 & 22.4 \\
\hline 0 & 30 & 0.0 & 0.0 & 0.0 & 45.8 & 45.4 & 45.6 & 0.4 & 0.0 & 0.2 & 62.9 & 0.0 & 31.4 & 57.9 & 0.0 & 28.9 & 74.4 & 0.8 & 37.6 & 15.3 & 32.7 & 24.0 \\
\hline$*_{0}$ & 45 & 2.8 & 2.5 & 2.7 & 54.2 & 54.2 & 54.2 & 0.4 & 0.0 & 0.2 & 63.1 & 0.0 & 31.5 & 58.8 & 0.0 & 29.4 & 68.6 & 0.8 & 34.7 & 19.0 & 31.9 & 25.5 \\
\hline 0 & 60 & 22.1 & 20.6 & 21.3 & 55.4 & 55.4 & 55.4 & 3.7 & 2.9 & 3.3 & 64.0 & 0.0 & 32.0 & 63.6 & 0.0 & 31.8 & 60.0 & 0.7 & 30.3 & 26.7 & 31.4 & 29.0 \\
\hline 0 & 75 & 34.8 & 27.9 & 31.3 & 53.4 & 53.4 & 53.4 & 20.7 & 18.7 & 19.7 & 71.9 & 0.2 & 36.1 & 66.7 & 0.0 & 33.3 & 57.1 & 1.0 & 29.0 & 34.8 & 32.8 & 33.8 \\
\hline 0 & 90 & 29.9 & 23.7 & 26.8 & 48.3 & 45.7 & 47.0 & 27.4 & 24.9 & 26.1 & 68.1 & 0.0 & 34.0 & 62.4 & 0.0 & 31.2 & 53.6 & 0.7 & 27.2 & 33.3 & 30.8 & 32.1 \\
\hline 15 & 0 & 0.0 & 0.0 & 0.0 & 41.7 & 39.8 & 40.7 & 0.4 & 0.0 & 0.2 & 86.2 & 0.7 & 43.5 & 81.0 & 0.2 & 40.6 & 70.6 & 0.1 & 35.3 & 13.6 & 39.8 & 26.7 \\
\hline 15 & 15 & 0.0 & 0.0 & 0.0 & 48.6 & 48.1 & 48.4 & 0.4 & 0.0 & 0.2 & 84.8 & 1.2 & 43.0 & 79.8 & 0.2 & 40.0 & 77.2 & 0.4 & 38.8 & 16.2 & 40.6 & 28.4 \\
\hline 15 & 30 & 0.8 & 0.8 & 0.8 & 57.1 & 57.1 & 57.1 & 0.4 & 0.0 & 0.2 & 81.4 & 1.0 & 41.2 & 78.3 & 0.2 & 39.3 & 76.3 & 0.7 & 38.5 & 19.4 & 39.6 & 29.5 \\
\hline 15 & 45 & 17.4 & 15.9 & 16.7 & 62.5 & 62.5 & 62.5 & 2.8 & 2.6 & 2.7 & 81.9 & 1.0 & 41.4 & 79.3 & 0.2 & 39.8 & 74.6 & 0.8 & 37.7 & 27.3 & 39.6 & 33.5 \\
\hline 15 & 60 & 37.0 & 28.0 & 32.5 & 62.5 & 62.5 & 62.5 & 20.4 & 18.3 & 19.3 & 85.2 & 1.0 & 43.1 & 85.0 & 0.2 & 42.6 & 67.6 & 0.8 & 34.2 & 38.1 & 40.0 & 39.1 \\
\hline 15 & 75 & 32.7 & 25.1 & 28.9 & 57.4 & 57.4 & 57.4 & 28.4 & 25.1 & 26.8 & 88.1 & 0.7 & 44.4 & 88.6 & 0.2 & 44.4 & 59.9 & 0.8 & 30.3 & 37.7 & 39.7 & 38.7 \\
\hline 15 & 90 & 41.7 & 25.0 & 33.3 & 54.6 & 49.7 & 52.2 & 59.6 & 18.9 & 39.2 & 83.8 & 0.0 & 41.9 & 84.0 & 0.2 & 42.1 & 56.9 & 0.1 & 28.5 & 41.6 & 37.5 & 39.6 \\
\hline 30 & 0 & 0.0 & 0.0 & 0.0 & 51.7 & 50.9 & 51.3 & 0.4 & 0.0 & 0.2 & 96.7 & 2.1 & 49.4 & 93.6 & 0.2 & 46.9 & 69.3 & 0.3 & 34.8 & 17.2 & 43.7 & 30.4 \\
\hline 30 & 15 & 0.1 & 0.0 & 0.0 & 61.6 & 61.6 & 61.6 & 0.4 & 0.0 & 0.2 & 96.2 & 2.1 & 49.2 & 94.8 & 0.5 & 47.6 & 74.3 & 0.8 & 37.6 & 20.6 & 44.8 & 32.7 \\
\hline 30 & 30 & 13.3 & 11.9 & 12.6 & 67.4 & 67.4 & 67.4 & 3.2 & 2.5 & 2.9 & 95.5 & 1.9 & 48.7 & 94.0 & 0.2 & 47.1 & 77.6 & 0.7 & 39.2 & 27.6 & 45.0 & 36.3 \\
\hline 30 & 45 & 37.1 & 27.3 & 32.2 & 68.7 & 68.7 & 68.7 & 20.1 & 17.1 & 18.6 & 95.5 & 1.9 & 48.7 & 92.1 & 0.2 & 46.2 & 76.8 & 0.8 & 38.8 & 39.8 & 44.6 & 42.2 \\
\hline 30 & 60 & 34.6 & 26.3 & 30.5 & 64.8 & 64.8 & 64.8 & 27.7 & 23.5 & 25.6 & 97.9 & 2.1 & 50.0 & 96.0 & 0.2 & 48.1 & 67.9 & 0.8 & 34.4 & 40.3 & 44.2 & 42.2 \\
\hline 30 & 75 & 41.7 & 23.9 & 32.8 & 59.6 & 59.6 & 59.6 & 57.1 & 17.2 & 37.1 & 96.7 & 1.0 & 48.8 & 95.0 & 0.5 & 47.7 & 63.9 & 0.3 & 32.1 & 43.2 & 42.9 & 43.0 \\
\hline 30 & 90 & 0.0 & 0.0 & 0.0 & 56.2 & 46.5 & 51.3 & 0.4 & 0.0 & 0.2 & 93.8 & 0.0 & 46.9 & 91.0 & 0.2 & 45.6 & 56.0 & 0.1 & 28.1 & 17.2 & 40.2 & 28.7 \\
\hline 45 & 0 & 0.0 & 0.0 & 0.0 & 61.7 & 61.7 & 61.7 & 0.4 & 0.0 & 0.2 & 99.3 & 1.7 & 50.5 & 94.5 & 0.5 & 47.5 & 64.7 & 0.7 & 32.7 & 20.6 & 43.6 & 32.1 \\
\hline 45 & 15 & 7.8 & 7.2 & 7.5 & 69.9 & 69.9 & 69.9 & 2.8 & 1.4 & 2.1 & 98.8 & 2.1 & 50.5 & 93.8 & 0.2 & 47.0 & 69.9 & 0.7 & 35.3 & 26.5 & 44.3 & 35.4 \\
\hline 45 & 30 & 36.5 & 27.5 & 32.0 & 71.8 & 71.8 & 71.8 & 16.9 & 13.5 & 15.2 & 99.8 & 2.1 & 51.0 & 94.8 & 0.5 & 47.6 & 75.0 & 1.0 & 38.0 & 39.6 & 45.5 & 42.6 \\
\hline 45 & 45 & 34.4 & 26.6 & 30.5 & 69.0 & 69.0 & 69.0 & 26.0 & 21.9 & 24.0 & 99.5 & 2.1 & 50.8 & 96.7 & 0.5 & 48.6 & 74.4 & 1.0 & 37.7 & 41.2 & 45.7 & 43.4 \\
\hline 45 & 60 & 41.3 & 24.0 & 32.7 & 63.9 & 63.9 & 63.9 & 54.6 & 15.5 & 35.0 & 99.8 & 2.1 & 51.0 & 95.7 & 0.5 & 48.1 & 67.6 & 0.4 & 34.0 & 43.9 & 44.4 & 44.1 \\
\hline 45 & 75 & 0.0 & 0.0 & 0.0 & 59.7 & 57.1 & 58.4 & 0.4 & 0.0 & 0.2 & 98.3 & 0.0 & 49.2 & 95.2 & 0.2 & 47.7 & 9.0 & 0.0 & 4.5 & 19.5 & 33.8 & 26.7 \\
\hline 45 & 90 & 0.0 & 0.0 & 0.0 & 60.3 & 35.6 & 48.0 & 0.4 & 0.0 & 0.2 & 97.4 & 0.0 & 48.7 & 93.1 & 0.5 & 46.8 & 49.6 & 0.1 & 24.9 & 16.1 & 40.1 & 28.1 \\
\hline \multicolumn{2}{|c|}{ DIP-fixed } & 0.0 & 0.0 & 0.0 & 20.7 & 0.0 & 10.3 & 0.0 & 0.0 & 0.0 & 61.7 & 0.0 & 30.8 & 50.7 & 0.0 & 25.4 & 53.9 & 0.0 & 26.9 & 3.4 & 27.7 & 15.6 \\
\hline \multicolumn{2}{|c|}{$\mathrm{ABD}$} & 0.4 & 0.3 & 0.3 & 44.1 & 44.1 & 44.1 & 0.0 & 0.0 & 0.0 & 64.3 & 0.0 & 32.1 & 62.6 & 0.0 & 31.3 & 69.3 & 0.1 & 34.7 & 14.8 & 32.7 & 23.8 \\
\hline
\end{tabular}



Discussion 



\section{General discussion}

In this doctoral thesis, I have proposed experimental and analytical methods, which can be useful for the design and evaluation of anthropomorphic artificial hands. In parallel, a new design of a TDPH, named IMMA hand, was proposed in Chapter 1 and was analyzed through this thesis using the different methods proposed. It has six DoFs actuated by tendons, two of them for thumb opposition (flexion and circumduction), and combines different materials to obtain an appropriate friction coefficient and compliance in the contact areas with the objects. Its grasping ability was evaluated experimentally in Chapter 3 using the benchmark AHAP defined in Chapter 2 and an ABA, and good results were obtained in comparison to other artificial hands with similar characteristics. In addition, it was determined that two principal components explain near $80 \%$ of the variance in tendon motions for this hand during the experiments under human actuation, providing a good starting point for the design of a simple actuation and control system. In Chapters 4 and 5 complementary analytical indexes were defined enabling the assessment of the anthropomorphism of the IMMA hand and other artificial hands. In Chapters 6 and 7 the optimal design for the kinematic chain of the thumb and some other design alternatives were analyzed for this hand using simulation models, either with a new optimization procedure based on the performance for a good thumbto-fingers opposition or with a preliminary benchmark, based on the AHAP, within a grasping simulation environment. The different analytical and experimental methods proposed in this thesis have allowed to compare the IMMA hand against others and to identify some of its limitations and features to improve.

One of the conclusions of the thesis is that a combination of experimental, analytical and simulated methods is a good strategy for the evaluation of hand designs and prototypes.

Experimentation is time-consuming, but it is useful to have a better insight into the actual grasping performance on hand prototypes, because it is difficult to model accurately factors such as contact friction or deformation and other dynamical effects. Most of the previous studies evaluating artificial hands include preliminary grasping tests that are difficult to compare with those performed by other research groups, because they are not based on clearly established protocols or different objects or metrics are used. Recent reviews on benchmarks and testing methods in the fields of prosthetics (Mio 


\section{8 | Discussion}

et al., 2018) and robotics (Quispe et al., 2018) emphasize the relevance of evaluating functional abilities of artificial hands with standardized testing methodologies. The increase of artificial hand designs for prosthetics, service robotics and human-robot cooperation applications, also requires that these tests try to evaluate the grasping ability from an anthropomorphic perspective, i.e. considering the comparison with the grasps and strategies used by humans. Moreover, in order to be more useful for design, the evaluation methods should be able to elucidate the effect of different design factors, such as mechanical design, actuation or control, in the final performance. Chapters 1, 2 and 3 address properly these concerns. In Chapter 1 , I proposed a preliminary study of an experimental protocol to evaluate grasping ability of TDPHs using an actuation device to control the artificial hand manually with the fingers of a healthy operator. The manual operation of the tendons allows focusing the assessment on the mechanical design of the artificial hand. In this way, the haptic and proprioceptive feedback to the healthy operator allows improving the control of the artificial hand while grasping. Additionally, the device (ABA) presented provided information about the excursion of the tendons during the grasp of the different objects used in the protocol. This information can be very useful for designing the motorized actuation and the control strategy of underactuated hands. From the preliminary tests performed in Chapter 1 , some limitations were observed in the design of the ABA, which was improved in Chapter 3. The initial protocol presented in Chapter 1 was later improved to make it more comprehensive, repeatable and consistent, as presented in Chapter 2. The results of the IMMA hand obtained with the different versions of the protocol, shown in Chapter 1 and Chapter 3, are different due to the nonidentical execution procedure and scoring system used in each version. It can be noted that the AHAP is stricter than the preliminary one (Chapter 1), with a reduction of about $16 \%$ in the total score of the IMMA hand, mainly due to the detailed definition of the grasp type correctness in the AHAP version. From this experimental tests some ideas arose about the need to optimize the orientation of the thumb joints for a further improved version of the IMMA hand, leading up to perform deep computational analyses of this feature in Chapters 6 and 7.

As a result of the work presented in Chapters 1 and 2 a universal experimental benchmark to evaluate the grasping ability of robotic and prosthetic anthropomorphic hands through the Grasping Ability Score (GAS) has been obtained: the Anthropomorphic Hand Assessment Protocol (AHAP). It solves some of the limitations observed in previous studies 
providing a standardized experimental assessment. It uses a publicly available set of objects and evaluates the ability to replicate the main human GTs and to perform stable grasps. Intra- and inter-rater reliability, consistency and responsiveness were analyzed verifying the robustness of the AHAP across raters and proving the comparability of the results also across different hands and testing conditions. In this thesis the AHAP has been applied to different hands, including three configurations of the hand of the humanoid robot ARMAR-6 (Asfour et al., 2018) and two versions of the KIT Prosthetic Hand (Weiner et al., 2018), as well as other 3D-printed TDPHs. The results of the AHAP can be individually analyzed for each GT and for the two parts of the experimental protocol, grasping and maintaining, allowing a detailed and precise representation of the hand's abilities. It is worth to note that this benchmark does not only provide a basis of comparison, but also a way to recognize possible design improvements.

In Chapter 3, the application of the AHAP involves a different approach to that of previous studies (Cipriani et al., 2008; Jamone et al., 2016) by evaluating the grasping ability of anthropomorphic artificial hands independently of their actuation or control system. It is relevant, because a fair comparison of one of the different design aspects such as the mechanical design of the hands, the actuation method or the control algorithms, is only possible when the other ones are fixed. In this chapter I present, to my knowledge, the first systematic comparison of the grasping ability of several low-cost 3D-printed TDPHs. I applied the AHAP with an improved version of the ABA presented in Chapter 1. The tendons of the artificial hands were actuated independently by the fingers of healthy human subjects, thus excluding from the comparison the specific control or actuation implementation with potential users. With this strategy, the comparison was focused on the mechanical design of the hands. The results highlight some of the limitations of current low-cost hands and also show the acceptable outcome for the IMMA hand. The fact that the subject's effect was found non-significant on both GAS and partial GAS evinces that a single subject could evaluate the hands using this improved ABA and the AHAP. However, an analysis of the day/subject/prosthesis effect is missing in this study, and thus it is not clear if the learning effect throughout the sessions could have affected the results.

Despite other ABAs have been applied previously in the literature for simulating the use of hand prostheses (Bouwsema et al., 2014; Dalley et al., 2012; Fougner et al., 2014; Huinink et al., 2016; Kyberd, 2011; Rossi et al., 2017; Smit et al., 2015; Vasluian et al., 2014), the ABA presented in this 


\section{0 | Discussion}

thesis is the only one including a system to actuate manually and independently the fingers of TDPHs and enabling to register the tendon excursions. It allows to take advantage of the human brain control while obtaining the synergies directly from the artificial hand, therefore summarizing the positive characteristics remarked by Salvietti (Salvietti, 2018). In addition, the manual actuation of the artificial hand by a human operator by means of the cables provides haptic and proprioceptive feedback that allows an improved control of the hand. Notwithstanding, its current design can be further improved in order to make it lighter and less bulky.

The underactuation is essential to simplify the control of the artificial hands, especially for prostheses and/or affordable designs. Chapter 3 presents, as far as I know, the first analysis about the synergies observed in tendon motion of artificial hands actively actuated and controlled by a human in a representative set of GTs in ADL. These synergies could be very useful for coupling the actuation of some DoFs of affordable prosthetic hands at the software level and/or the hardware level. Indeed, the synergies obtained are in accordance with other studies in the literature performed with the human hand (Gracia Ibañez, 2016; Santello et al., 1998) and also with the number of motors used in currently existing prostheses (Catalano et al., 2014; Huang et al., 2006; Weiner et al., 2018). However, some limitations of the results can be considered. One is that only three subjects where used to obtain the synergies, limiting the statistical significance. Other is the fact that these synergies are based only on tendon displacement, but information about the force sharing among fingers is not measured, which could provide a more comprehensive view. It is not clear to which extent a particular underactuation design to couple the tendon motion among fingers could be optimal for an adequate force sharing among fingers.

The AHAP is a powerful tool to evince the necessary improvements in the design of artificial hands to increase their functionality. In this sense, some of the results obtained in Chapters 2 and 3 are illustrative. For the ARMAR-6 v1 hand, the GAS increased (45\% - 55\% - 61\%) with the increasing addition of friction pads, indicating that the hand surface characteristics have a significant impact on the final performance. The improvements made in the finger kinematic chain of the last version of the KIT Prosthetic Hand allowed to improve the GAS from $72 \%$ in its first version to $79 \%$ in the final one. Among the TDPHs assessed with the ABA in Chapter 3, the IMMA hand scored the highest GAS (57\%). It is attributed mainly to the additional degree of actuation (DoA) for thumb circumduction and the selected materials for its different parts. Another conclusion obtained 
in this chapter is that the improvements on these hands should be especially focused on improving the grasp stability, because the results showed that maintaining the objects firmly grasped under motion was the most challenging task. For a successful execution of the extension grip, it would be useful to have extensor tendons or joints with different bending stiffness for keeping extended the distal segments of the fingers while flexing their metacarpophalangeal (MCP) joints. For the pulp pinch, lateral pinch, diagonal volar grip and tripod pinch, the orientation of the thumb to oppose to the long fingers should be optimized. With this purpose, specific computational methods were proposed and applied to the IMMA hand in Chapters 6 and 7. In addition to the thumb opposition, the palm opposition should be improved with more human-shaped designs of the palm for improving spherical, cylindrical and diagonal volar grips. This better shaped palm could explain the clearly higher GAS scored by the KIT prosthetic hand with respect to the other hand models tested with the AHAP in this thesis. The partial GAS obtained by this hand in pulp pinch, diagonal volar grip, cylindrical grip, spherical grip and extension grip is the highest observed among the hands tested. The reachable grasping force is limiting for power grasps as was observed for heavy objects. Decreasing the friction of the tendons along their path should improve this grasping force. Moreover, the selection of materials with good compliance and friction coefficient for the hand-object contact areas is a key point to improve the stability of these grasps, as deduced from the better results obtained by the IMMA hand, the ARMAR-6 v1 hand supplemented with friction pads, and the KIT prosthetic hand which uses PEBA2301 material in the distal phalanges.

According to the definition of Falco et al. (Falco et al., 2015) the AHAP can be considered as a functional test and could complement some other component and system experimental tests proposed in the literature (Falco et al., 2015; Mio et al., 2018; Quispe et al., 2018). Additionally, experimental methods can be complemented with analytical or computational methods, which could help to estimate and maximize the degree of anthropomorphism of artificial hands in the initial design stages. Analytical and computational methods are less expensive and generally its application requires less time than experimental methods. Because of this, some new analytical and computational methods have been proposed in Chapters 4 to 7 of this thesis.

In Chapters 4 and 5 different anthropomorphism indexes were proposed. The Anthropomorphism Index of Mobility (AIM) proposed in Chapter 4 is based just on information about the number of DoFs of the artificial hand and the possibility to control them independently. It was primarily focused 


\section{2 | Discussion}

on prosthetic hands but finally it was validated for artificial hands both robotic and prosthetic, regardless of whether they are dominant or nondominant hands. Its use in the concept design stage is justified because its calculation is really quick. Despite the simple computation, it analyzes not only the topology but also the functionality of the artificial hand, because it takes into account results obtained in grasping tests with the human hand about the relevance of the different DoFs for the mobility of the hand in ADL. Notwithstanding, some important design factors as the orientation of the joints axes, the range of motion of the joints or the dimension of the phalanges, are not considered in the AIM and should be taken into account in later design stages, as preliminary or detail design. Therefore, in Chapter 5, three different Anthropomorphism Indexes of the Kinematic Chain (AIKCs) were proposed, based on the comparison of: 1) the parameters of the kinematic chain (dimensions, type of joints, orientations and ranges of motion), 2) the reachable workspace, and 3) common grasping postures. These indexes are of valuable help to evaluate how far is the kinematic chain of the artificial hand from that of the human hand. They are computed based on simplified models of the hands considering lines for representing the hand bones. However, the simplifications taken for the human hand model or for the synergistic motions in both artificial and human hands does not limit the relevance of this contribution, because the proposed methodology can be maintained after improvements in these simplifications. A clear strategy about how to combine the proposed indexes to make design decisions has not been tackled in the thesis and it is object for future work. Anyway, a hand scoring better in all these indexes is expected to be more anthropomorphic in the motions performed and the reachable positions.

Furthermore, the work presented in Chapter 4 includes an important contribution about the relevance of the different groups of DoFs of the human grasps in ADL, obtained from a human grasp experiment. These results indicate that finger flexion-extension $(\mathrm{F} / \mathrm{E})$ is by far the most relevant group of DoFs accounting for more than half of the functionality (55\%), followed by thumb opposition (T.OPP) (24\%) and finger abductionadduction (16\%). The palmar arching has a relevance of only 5\%. This relevance by itself is valuable for making decisions during the design of new artificial hands to maximize their functionality. It is worth to note that this result is coherent with the groups of DoFs mainly included and actively driven in the artificial hands, fingers $\mathrm{F} / \mathrm{E}$ and thumb opposition.

The indexes proposed are also useful to evaluate and compare the anthropomorphism of artificial hands, as has been done in this thesis. The 
most advanced robotic hands (DLR/HIT II and Shadow) with a significant amount of DoFs and motors located in the important groups of DoFs obtained the highest AIM scores, above $75 \%$. The commercial prosthetic hands i-Limb and Bebionic as well as some 3D-printed hands (ADA, IMMA) obtained AIM scores between $40 \%$ and $50 \%$ because they include a reasonable number of motors and DoFs in the F/E and T.OPP groups. The SensorHand obtained the lowest score due to the improvable number of DoFs, motors, or type of underactuation in the groups of F/E, T.OPP, or both. The artificial hands analyzed with other anthropomorphism indexes of the literature, such as the Anthropomorphism Index of Feix et al. (Feix et al., 2013b) and the Total Score of Anthropomorphism of Liarokapis et al. (Liarokapis et al., 2013), are ranked equally by the AIM and the other metrics, although the scores are different due to the different definition of the indexes. Among the hands compared in this thesis using the AIKCs, the Shadow robotic hand presented the highest scores. The advanced commercial prosthesis i-Limb presents AIKCs comparable to other affordable artificial hands. The AIKCs for the three-finger Barrett robotic hand are lower, although the $A I K C_{2}$ score is comparable to that of other fivefingered hands. The indexes proposed in Chapter 5 to evaluate the anthropomorphism of the kinematic chain go beyond other indexes proposed in the literature for this objective, because they consider all the parameters defining the kinematic chain, that is, the position and orientation of all the joints including all the finger segments. Feix et al. (Feix et al., 2013b) only included the fingertip position and orientation to define the reachable workspace of the hand and Liarokapis et al. (Liarokapis et al., 2013) considered the position of other joint centers but the orientation only for the MCP joints. Moreover, in this thesis, a method to compare the joint orientations by means of quaternions (Huynh, 2009) is used. This method is more accurate than using Euler angles (Liarokapis et al., 2013) or rotation matrices (Feix et al., 2013b). The use of alpha-shapes proposed in Chapter 5 as an alternative to convex-hulls to compare the workspace avoid the inclusion of interior regions or other areas that do not strictly pertain to the reachable workspace (Liarokapis et al., 2013). An interesting result obtained in Chapter 5 is that the combination of abduction/adduction with flexion/extension movements in the same finger is what allows the human hand to increase the volume of its workspace. In fact, the Shadow hand achieves a higher $A I K C_{2}$ because its MCP joints include these both DoFs. The results obtained with AIKCs are not directly comparable with those of Feix et al. (Feix et al., 2013b), since different hands were analyzed, although the orders of magnitude were similar to those obtained with $A I K C_{2}$. Feix et 


\section{4 | Discussion}

al. obtained a value of 5.2\%-9.2\% (depending on the sampling method) for the hand with the highest number of DoFs analyzed (FRH-4) and $0.25 \%$ for the prosthesis SensorHand with three fingers, which is comparable to the Barrett hand. In Liarokapis et al. (Liarokapis et al., 2013) the score for Shadow (40\%) was higher than for Barrett (10\%) as in our results, although the values are higher than those obtained in $A I K C_{2}$ because they are based on the comparison of workspace volumes using convex-hulls instead of alpha-shapes. Comparing the results obtained with the AIM (Chapter 4) and the AIKCs (Chapter 5) it is deduced some agreement between both analyses, because the Shadow and the Barrett hands are the most and the least anthropomorphic respectively. However, IIKC $_{1}$ and AIM rank the IMMA and i-Limb differently, and the Shadow exhibits higher AIM values (almost double) compared to the previous ones, while for the $A I K C_{1}$ and $A I K C_{3}$ these differences are lower than $10 \%$. This discrepancy indicates that the differences among these hands are higher in terms of actuation mobility that just in terms of their kinematic chain. The number of actuators and type of underactuation, considered in the AIM definition, were not taken into account in the AIKCs.

A comparison between the results obtained with the experimental method and analytical methods proposed in this thesis can be done only partially because not all the hands were evaluated with the different methods. In fact, only GAS and AIM scores can be compared for the IMMA and KIT prosthetic hands. The IMMA hand obtained a better result in AIM while the KIT prosthetic hand scored better according to the GAS obtained from the experimental AHAP. This discrepancy is attributed to better design solutions of the KIT prosthetic hand in comparison to the IMMA hand, such as the better shape of the different parts of the hand, the better friction coefficient and the higher efficiency of the transmission. These factors have an effect in the experimental evaluation but were not taken into account in the analytical index. The different actuation method used in both hands when performing the AHAP could also have had an effect on the GAS score.

In Chapters 6 and 7, I explored computational methods useful to obtain optimal hand design alternatives. These methods were mainly applied to evaluate the kinematic chain of the thumb, given its relevance for a good opposition, and they were tested on the IMMA hand.

In Chapter 6 a new method to optimize the thumb kinematic chain of an artificial hand based on their performance in the Kapandji opposition test (KOT) (Kapandji, 1986) was presented. This method follows a formal 
optimization procedure that allows including all the parameters defining the kinematic chain. An index quantifying the mean weighted position error (MPE) of the different KOT postures was proposed as the optimization parameter. Due to the simplification of the hand model, whose segments are considered as straight lines, the computational model is only an approximation to the real prototype. This led to some disagreement between the reachability of some of the Kapandji postures (1 to 4) experimentally and the small non-null position errors obtained for these postures with the model. This disagreement was attributed to the fact that the width and thickness of the phalanges were neglected in the simplified model and to the specific point considered for postures 1 and 2 of the KOT. Taking these considerations, the improved model obtained with the optimization method could achieve up to position 8 of the KOT, and the MPE was reduced to less than one third of that of the original design. However, the computation of the AIKCs presented in Chapter 5 for the IMMA hand with both thumb designs, original and optimized, suggests that the reduction of MPE in KOT does not guarantee a better design according to other criteria when comparing with the human hand. The performance was similar, with differences lower to 3\%, for $A I K C_{1}$ and $A I K C_{3}$, but for $A I K C_{2}$ the optimized design had a worse performance, $25 \%$ lower. This contradictory result is aligned with the observation of Roa et al. (Roa et al., 2014) about the difficulties to find direct correlations between the KOT score and the size of the functional workspace.

Chapter 7 is focused on evaluating and comparing artificial hands according to their anthropomorphism on the simulated grasps performed using simulation tools. In this sense, the approach can be considered as an extension of the method proposed for $\mathrm{AIKC}_{3}$ in Chapter 5 . However, here the use of OpenRAVE allows a better representation of the hand and object geometries and the contact models and metrics included in this grasping simulation framework can be exploited to have a better insight into the grasp stability. Some attempts were made in the past to use this simulation environment for the evaluation of artificial hands (Rubert et al., 2017; Rubert and Morales, 2016), using a brute-force approach for generating grasp hypotheses and grasp quality metrics for the evaluation. However, the results obtained in this chapter using that methodology present some limitations because the best grasps obtained do not fulfill the criteria of anthropomorphism defined in the literature (Sollerman and Ejeskär, 1995; Vergara et al., 2014) and were not realistic for the expected use of the objects by a human in ADL. Thus, a preliminary simulation benchmark following a different approach was proposed. It moves from the brute-force approach for 


\section{6 | Discussion}

generating the grasp hypotheses to a more human oriented generation and from the use of quality metrics for the evaluation to a scoring system based on the experimental AHAP (Chapter 2). It has been proved that the new method leads to final grasps more meaningful in terms of anthropomorphism and ADL. Additionally, it is more efficient in terms of computation time. This simulation benchmark allows evaluating anthropomorphic grasps in order to select optimal hand design alternatives. A noteworthy advantage is the reduced time required to compare different hand design alternatives against the experimental approach. In this study, thirty design alternatives of the IMMA hand were analyzed easily, while an experimental comparison for such a big number of alternatives would be unaffordable in a reasonable time. Notwithstanding, some limitations remain, suggesting that further improvement in the simulation is needed. In effect, the results obtained with the original IMMA hand with this simulation benchmark and those obtained experimentally with the AHAP in Chapter 3 agree only partially. The discrepancy is mainly due to the method used in OpenRAVE to simulate the closing motion.

\section{References}

Asfour, T., Kaul, L., Wachter, M., Ottenhaus, S., Weiner, P., Rader, S., Grimm, R., Zhou, Y., Grotz, M., Paus, F., Shingarey, D., Haubert, H., 2018. ARMAR6: A Collaborative Humanoid Robot for Industrial Environments, in: 2018 IEEE-RAS 18th International Conference on Humanoid Robots (Humanoids), pp. 447-454.

Bouwsema, H., van der Sluis, C.K., Bongers, R.M., 2014. Changes in performance over time while learning to use a myoelectric prosthesis. Journal of NeuroEngineering and Rehabilitation, 11(1), 16.

Catalano, M.G., Grioli, G., Farnioli, E., Serio, A., Piazza, C., Bicchi, A., 2014. Adaptive synergies for the design and control of the Pisa/IIT SoftHand. The International Journal of Robotics Research, 33(5).

Cipriani, C., Zaccone, F., Micera, S., Carrozza, M.C., 2008. On the Shared Control of an EMG-Controlled Prosthetic Hand: Analysis of User-Prosthesis Interaction. IEEE Transactions on Robotics, 24(1), 170-184.

Dalley, S.A., Bennett, D.A., Goldfarb, M., 2012. Preliminary functional assessment of a multigrasp myoelectric prosthesis, in: 2012 Annual International Conference of the IEEE Engineering in Medicine and Biology Society, pp. 4172-4175.

Falco, J., Van Wyk, K., Liu, S., Carpin, S., 2015. Grasping the Performance: Facilitating Replicable Performance Measures via Benchmarking and 
Standardized Methodologies. IEEE Robotics \& Automation Magazine, 22(4), 125-136.

Feix, T., Romero, J., Ek, C.H., Schmiedmayer, H.B., Kragic, D., 2013. A metric for comparing the anthropomorphic motion capability of artificial hands. IEEE Transactions on Robotics, 29(1), 82-93.

Fougner, A.L., Stavdahl, Ø., Kyberd, P.J., 2014. System training and assessment in simultaneous proportional myoelectric prosthesis control. Journal of NeuroEngineering and Rehabilitation, 11(1), 75.

Gracia Ibañez, V., 2016. Contribution to hand functional assessment based on its kinematics. Ph.D., Universitat Jaume I, Castelló de la Plana.

Huang, H., Jiang, L., Zhao, D.W., Zhao, J.D., Cai, H.G., Liu, H., Meusel, P., Willberg, B., Hirzinger, G., 2006. The development on a new biomechatronic prosthetic hand based on under-actuated mechanism, in: IEEE International Conference on Intelligent Robots and Systems. pp. 3791-3796.

Huinink, L.H.B., Bouwsema, H., Plettenburg, D.H., van der Sluis, C.K., Bongers, R.M., 2016. Learning to use a body-powered prosthesis: changes in functionality and kinematics. Journal of NeuroEngineering and Rehabilitation, 13(1), 90.

Huynh, D.Q., 2009. Metrics for 3D rotations: Comparison and analysis. Journal of Mathematical Imaging and Vision, 35(2), 155-164.

Jamone, L., Bernardino, A., Santos-Victor, J., 2016. Benchmarking the Grasping Capabilities of the iCub Hand With the YCB Object and Model Set. IEEE Robotics and Automation Letters, 1(1), 288-294.

Kapandji, A., 1986. Clinical opposition and reposition test of the thumb. Annales de Chirurgie de la Main, 5(1), 67-73.

Kyberd, P.J., 2011. The influence of control format and hand design in single axis myoelectric hands: assessment of functionality of prosthetic hands using the Southampton Hand Assessment Procedure. Prosthetics and Orthotics International, 35(3), 285-293.

Liarokapis, M. V., Artemiadis, P.K., Kyriakopoulos, K.J., 2013. Quantifying anthropomorphism of robot hands, in: 2013 IEEE International Conference on Robotics and Automation, pp. 2041-2046.

Mio, R., Sanchez, M., Valverde, Q., 2018. Mechanical Testing Methods for BodyPowered Upper-Limb Prostheses: A Review, in: 2018 IEEE 18th International Conference on Bioinformatics and Bioengineering (BIBE), pp. 170-176.

Quispe, A.H., Amor, H. Ben, Christensen, H.I., 2018. A Taxonomy of Benchmark Tasks for Robot Manipulation, in: Springer Proceedings in Advanced Robotics. pp. 405-421.

Roa, M.A., Chen, Z., Staal, I.C., Muirhead, J.N., Maier, A., Pleintinger, B., Borst, 


\section{8 | Discussion}

C., Lii, N.Y., 2014. Towards a functional evaluation of manipulation performance in dexterous robotic hand design, in: 2014 IEEE International Conference on Robotics and Automation (ICRA), pp. 6800-6807.

Rossi, M., Della Santina, C., Piazza, C., Grioli, G., Catalano, M., Bicchi, A., 2017. Preliminary results toward a naturally controlled multi-synergistic prosthetic hand, in: 2017 IEEE International Conference on Rehabilitation Robotics (ICORR), pp. 1356-1363.

Rubert, C., León, B., Morales, A., Sancho-Bru, J., 2017. Characterisation of Grasp Quality Metrics. Journal of Intelligent \& Robotic Systems, 89(3-4), 319-342.

Rubert, C., Morales, A., 2016. Comparison between grasp quality metrics and the anthropomorphism index for the evaluation of artificial hands, in: 2016 6th IEEE International Conference on Biomedical Robotics and Biomechatronics (BioRob), pp. 1352-1357.

Salvietti, G., 2018. Replicating Human Hand Synergies Onto Robotic Hands: A Review on Software and Hardware Strategies. Frontiers in Neurorobotics, 12(JUN).

Santello, M., Flanders, M., Soechting, J.F., 1998. Postural hand synergies for tool use. The Journal of neuroscience: the official journal of the Society for Neuroscience, 18(23), 10105-10115.

Smit, G., Plettenburg, D., Van der Helm, F., 2015. The Lightweight Delft Cylinder Hand, the First Multi-Articulating Hand That Meets the Basic User Requirements. IEEE Transactions on Neural Systems and Rehabilitation Engineering, 23(3), 431-440.

Sollerman, C., Ejeskär, A., 1995. Sollerman Hand Function Test: A Standardised Method and its Use in Tetraplegic Patients. Scandinavian Journal of Plastic and Reconstructive Surgery and Hand Surgery, 29(2), 167-176.

Vasluian, E., Bongers, R.M., Reinders-Messelink, H.A., Burgerhof, J.G.M., Dijkstra, P.U., Sluis, C., 2014. Learning effects of repetitive administration of the Southampton Hand Assessment Procedure in novice prosthetic users. Journal of Rehabilitation Medicine, 46(8), 788-797.

Vergara, M., Sancho-Bru, J.L., Gracia-Ibáñez, V., Pérez-González, A., 2014. An introductory study of common grasps used by adults during performance of activities of daily living. Journal of Hand Therapy, 27, 225-234.

Weiner, P., Starke, J., Hundhausen, F., Beil, J., Asfour, T., 2018. The KIT Prosthetic Hand: Design and Control, in: 2018 IEEE/RSJ International Conference on Intelligent Robots and Systems (IROS), pp. 3328-3334. 
Conclusions 



\section{Contributions}

1. A new 3D-printed tendon-driven hand prosthesis prototype (IMMA hand) has been developed and manufactured using selected materials for the different parts of the hand.

2. A new experimental, standardized and reproducible benchmark (AHAP) that has been statistically validated has been presented and tested with different prosthetic and robotic hands. The AHAP includes the most frequently used GTs in ADLs and a wide range of objects of an internationally available object set (YCB set). The AHAP evaluates the functionality and anthropomorphism of the achieved grasp through a quantitative reliable measure of the grasping ability (GAS). It is a validated tool that can be used to evaluate and compare different aspects of artificial hands: the mechanical design, the actuation system and the control strategy. It is useful to improve the grasping capabilities of future designs.

3. An experimental framework has been proposed to evaluate the mechanical design and functionality of tendon-driven artificial hands when controlled by a human operator with an able-bodied adaptor (ABA). The ABA provides information about the control strategy employed during grasping because it registers the excursion of the actuating tendons during the grasp action. The analysis of the synergies in the motion of the tendons showed that the actuation and control systems could be designed in order to couple some DoFs due to the important correlations observed. The scores of the principal components obtained can be useful for both the design of transmission systems to underactuate the hand and the design of the control system.

4. A systematic comparison of the grasping ability of several affordable 3D-printed TDPHs has been undertaken using the AHAP, and the hand design limitations for grasping with the main GTs have been highlighted.

5. The functionality of the different groups of DoFs of the hand was analyzed according to a human grasp experiment on twenty subjects with the four main GTs for personal autonomy in ADL.

6. An anthropomorphism index of mobility (AIM), that can be used in the concept design stage to evaluate and compare artificial hands based on the mobility provided by their actuation and transmission system to the different DoFs in comparison with their relevance in the 


\section{2 | Conclusions}

human hand, has been presented. The index can be valid for both prosthetic and robotic hands, dominant and nondominant hands.

7. Three alternatives for the definition of the degree of anthropomorphism of the kinematic chain of an artificial hand have been defined and compared, solving some limitations on the definition of previous indexes of the literature to evaluate the hand workspace.

8. A straightforward methodology to analytically optimize the kinematic chain of the thumb of an artificial hand based on the performance in the Kapandji opposition test (KOT) has been presented. The cost function defined for the optimization is a weighted mean position error (MPE) when trying to reproduce the KOT postures and can also be used as a metric to quantify thumb opposition.

9. Different simulation approaches focused on assessing artificial hand grasping have been compared with the aim of obtaining a useful metric to measure their anthropomorphism and functionality during ADLs. The new simulation benchmark proposed follows an anthropomorphic approach for defining the grasp hypotheses and for evaluating stability and human likeness. Its principles are adapted from the experimental benchmark AHAP and considers the GT correctness criteria according to human experience resulting in more anthropomorphic and realistic grasps. The metric associated allows the comparison of different hand design alternatives.

10.Some improvements have been recommended for different robotic and prosthetic hand designs using the experimental and analytical methods proposed in this thesis. The results obtained should be taken into account for the design of new prototypes in order to obtain improved designs that maximize their anthropomorphism and functionality.

\section{Future work}

Given the promising results obtained in this thesis, the most imminent research work to be carried out is:

1. Inclusion of all the GTs of the experimental AHAP in the preliminary simulation benchmark proposed in Chapter 7, in order to make it more representative of ADL, generation of GHs based on human hand data obtained by videogrammetry, and modification of the method used in OpenRAVE to simulate the closing motion in order to improve its predictive performance. 
2. Analysis of the complementarity of the analytical metrics proposed and definition of a strategy to combine them in order to obtain optimal designs.

3. Design and manufacture of an improved version of the IMMA hand using the results obtained with the experimental and analytical methods proposed in this thesis, in order to make it more anthropomorphic and functional.

4. Evaluation and comparison of the optimized design of the IMMA hand with respect to the original design using experimental benchmarks such as the AHAP.

5. Analysis of the motion and force synergies of the improved version of the IMMA hand using the ABA and the AHAP with a significant number of users, in order to design the actuation, transmission and control systems of a final design that could be implemented as a hand prosthesis in potential users.

6. Design and comparison of the optimized IMMA hand being actuated as a body-powered and myoelectric prosthesis with experimental benchmarks and including the evaluation of the user experience.

7. Definition of new grasping benchmarks aimed at the evaluation of the control system, including the grasping performance with more realistic execution constraints. 



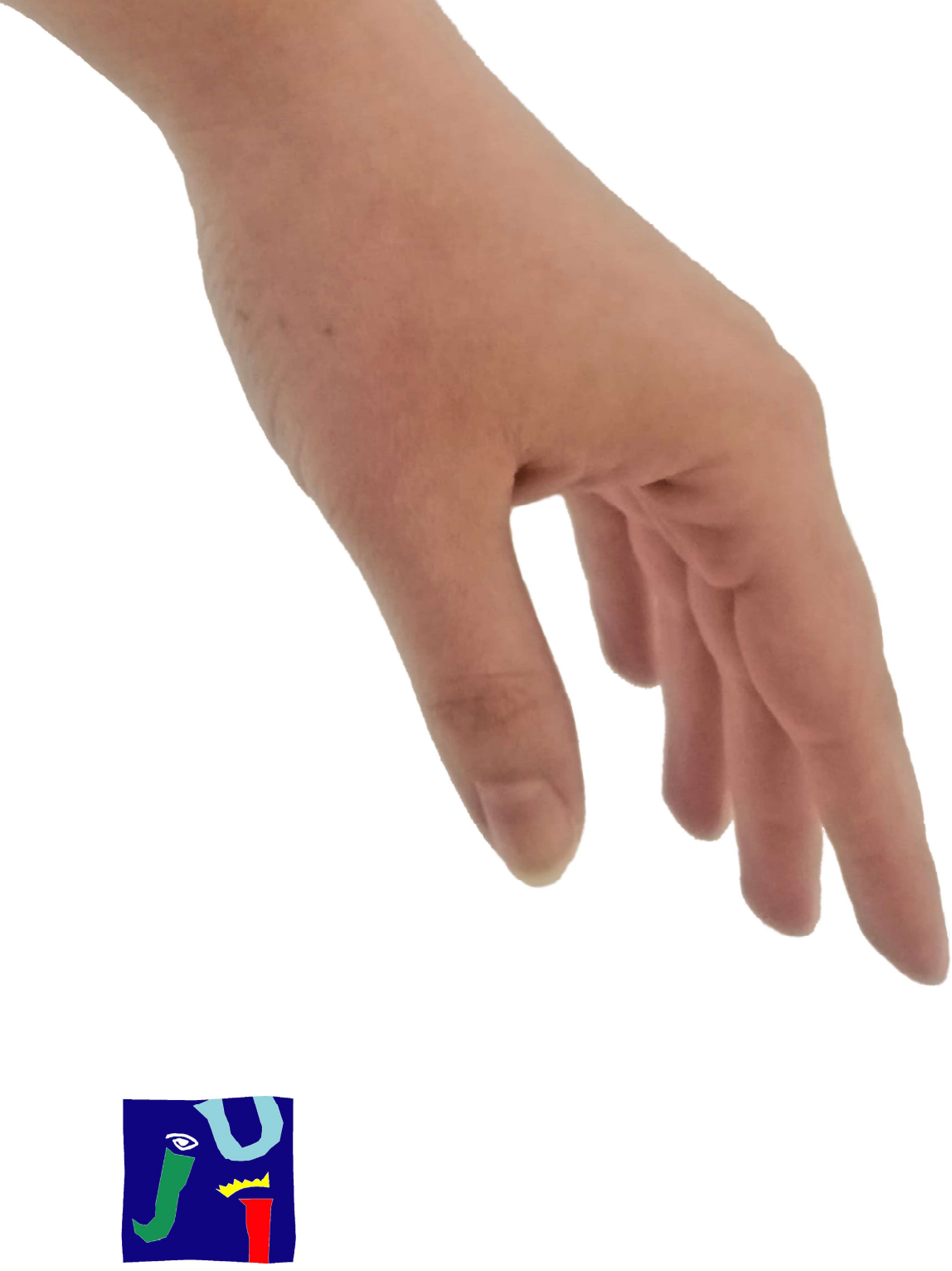

UNIVERSITAT

JAUME • I

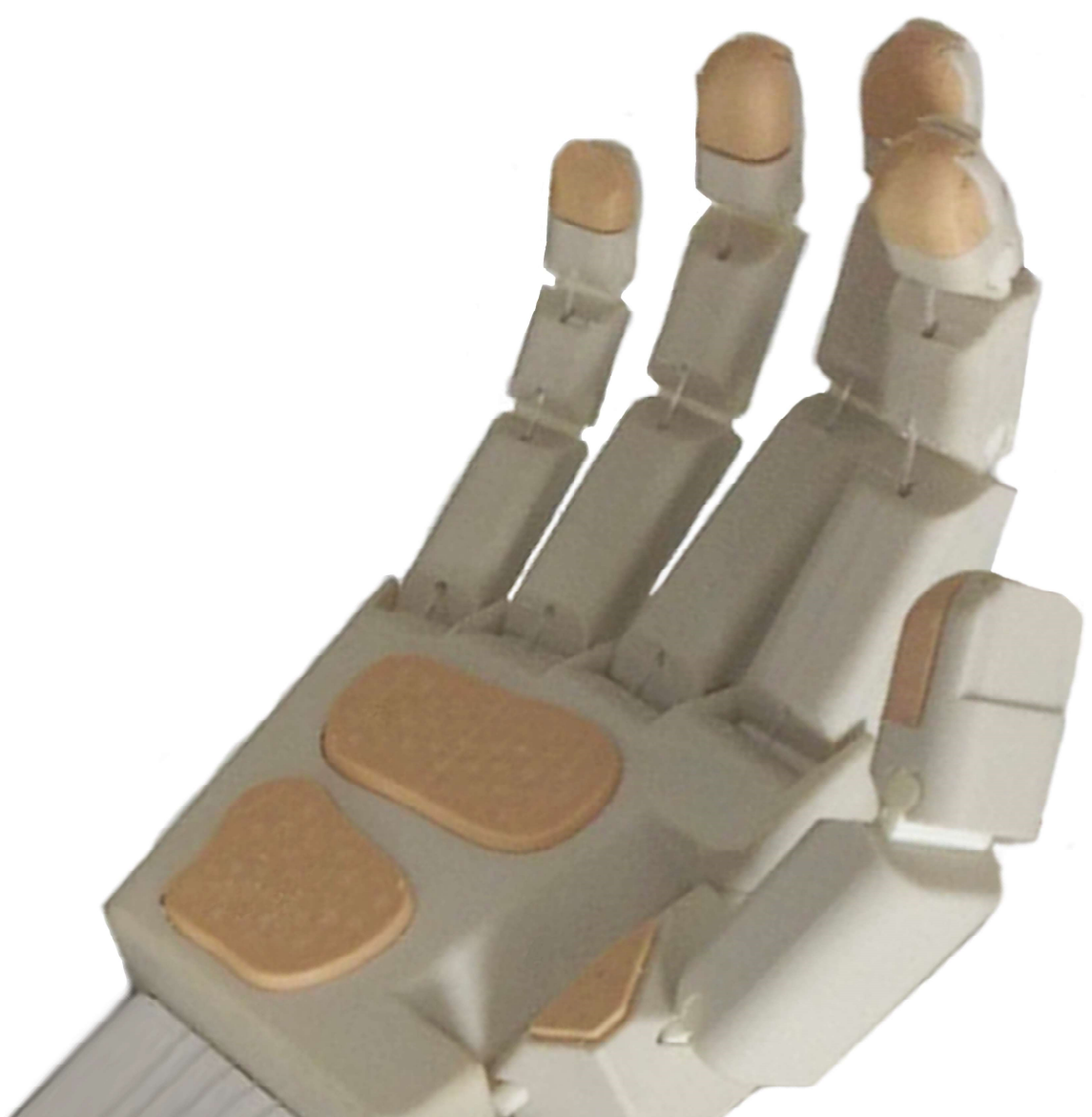

